\title{
A Role For Microtubule Dynamics For The Induction Of Chromosomal Instability And Cell Migration And Invasion In Human Cancer Cells
}

\section{Dissertation}

\author{
for the award of the degree \\ "Doctor rerum naturalium" \\ of the Georg-August-Universität Göttingen
}

within the doctoral program "Molecular Biology of Cells"

of the Georg-August University School of Science (GAUSS)

submitted by

Katharina Berger

from Brandenburg an der Havel, Germany

Göttingen, September 2016 


\section{Thesis Committee}

Prof. Dr. Holger Bastians

Institute for Molecular Oncology

Section of Cellular Oncology

University Medical Center Göttingen

Prof. Dr. Michael Thumm

Department of Cellular Biochemistry

University Medical Center Göttingen

Prof. Dr. Dieter Kube

Department of Haematology and Oncology

University Medical Center Göttingen

\section{Members of the Examination Board}

Referee: Prof. Dr. Holger Bastians

Institute for Molecular Oncology

Section of Cellular Oncology

University Medical Center Göttingen

$2^{\text {nd }}$ Referee: Prof. Dr. Dieter Kube

Department of Haematology and Oncology

University Medical Center Göttingen

\section{Further members of the Examination Board}

Prof. Dr. Michael Thumm

Department of Cellular Biochemistry

University Medical Center Göttingen

Prof. Dr. Matthias Dobbelstein

Institute for Molecular Oncology

University Medical Center Göttingen

Prof. Dr. Heidi Hahn

Department of Human Genetics

Section of Developmental Genetics

University Medical Center Göttingen

Prof. Dr. Peter Burfeind

Department of Human Genetics

University Medical Center Göttingen

\section{Date of oral examination}

18th of November 2016 


\section{Affidavit}

Hereby I declare that my doctoral thesis entitled "A Role For Microtubule Dynamics For The Induction Of Chromosomal Instability And Cell Migration And Invasion In Human Cancer Cells" has been written independently with no other sources and aids than quoted.

Göttingen, September 2016

Katharina Berger 


\section{Table Of Contents}

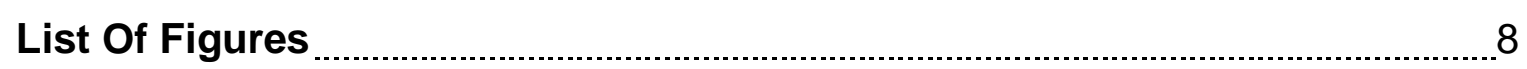

List Of Tables

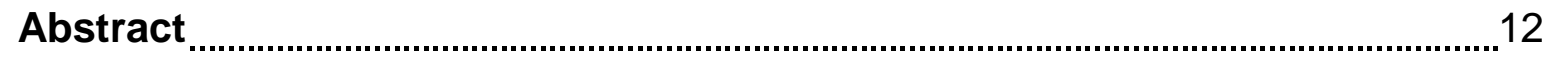

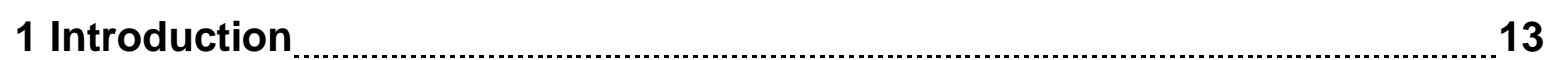

1.1 The Eukaryotic Cell Cycle .....................................................................................13

1.2 Cell Cycle Regulation And Checkpoints _......................................................... 14

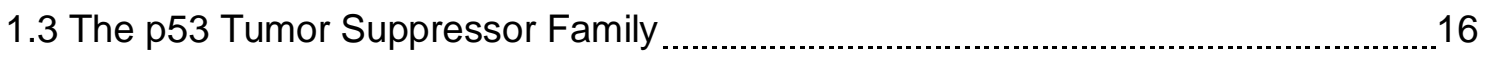

1.4 p21 As Mediator Of p53 Tumor Suppressor Activity _............................................. 17

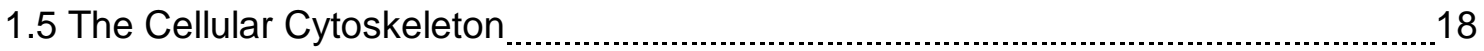

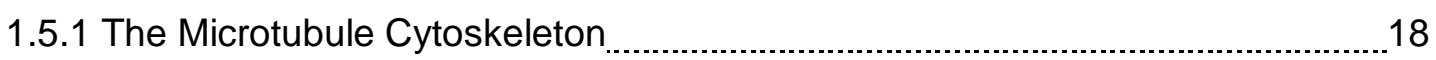

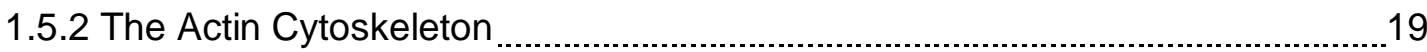

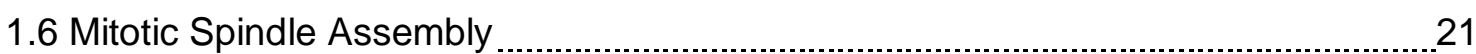

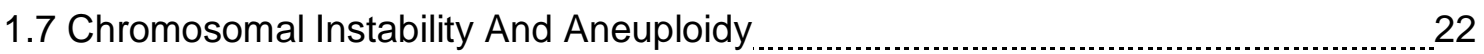

1.8 The Ras Homologous (Rho) Family Of Small GTPases _.........................................2

1.9 Cellular Migration

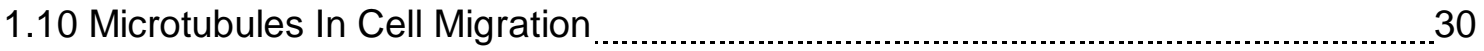

1.11 Cell Invasion

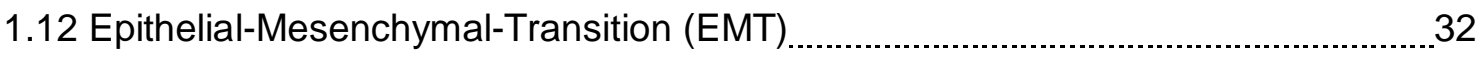

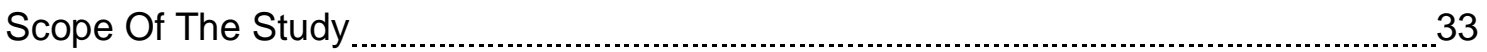

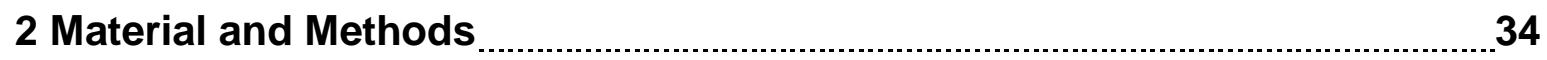

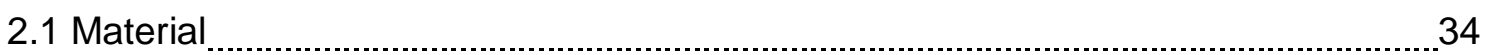

2.1.1 Equipment $\ldots \ldots \ldots \ldots$

2.1.2 Software 


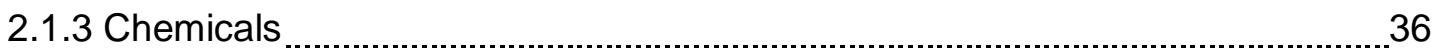

2.1.4 Antibodies 37

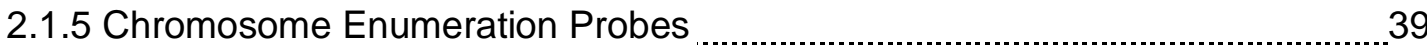

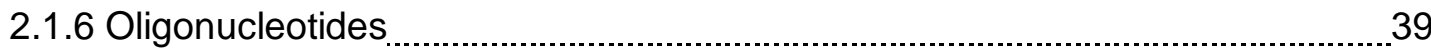

2.1.7 Plasmids $\quad 40$

2.1.8 Human Cell Lines

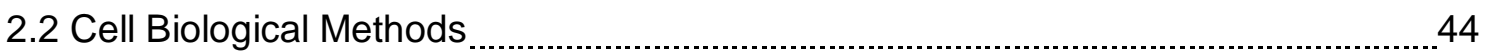

2.2.1 Transfection Of Human Cells 44

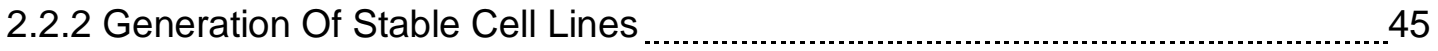

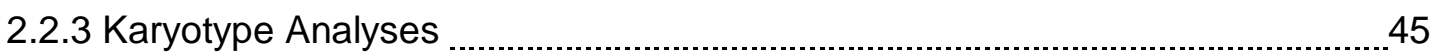

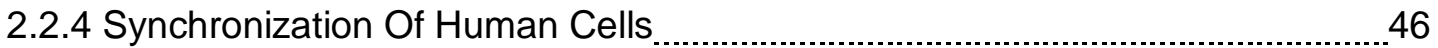

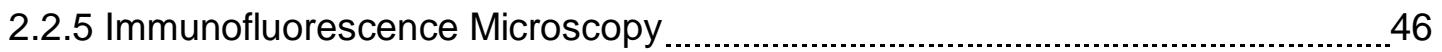

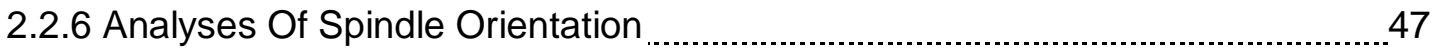

2.2.7 Analyses Of Microtubule Plus-End Assembly Rates ...................................... 47

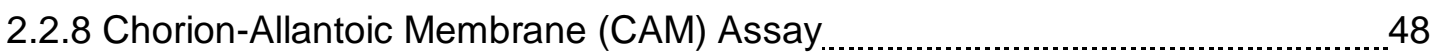

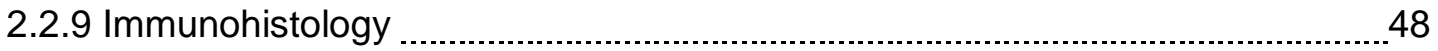

2.2.10 In Vitro Migration Assay ............................................................................. 48

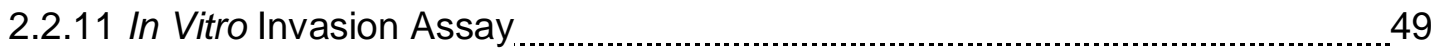

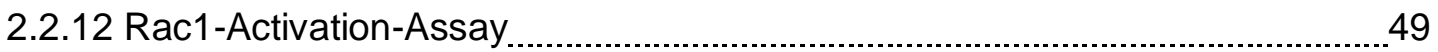

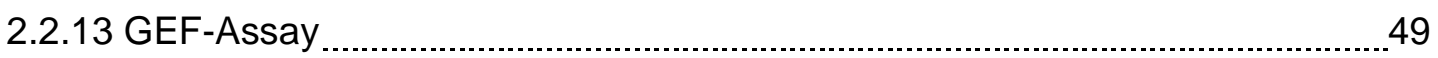

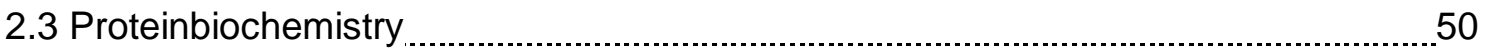

2.3.1 Preparation Of Protein Lysates

2.3.2 Protein Determination

2.3.3 Sodiumdodecylsulfate-Polyacrylamid Gel Electrophoresis (SDS-PAGE) ........50

2.3.4 Western Blot 
2.4 Molecular Biological Methods. 51

2.4.1 Escherichia coli Cells 51

2.4.2 Generation Of Competent Escherichia coli Cells 51

2.4.3 Transformation Of Escherichia coli Cells 52

2.4.4 Plasmid Isolation 52

2.4.5 RNA-Isolation 52

2.4.6 cDNA Synthesis And Quantitative Real Time PCR 52

2.4.7 Preparation of Samples For RNA-Sequencing 53

3 Results 54

3.1 A Mechanistic Link Between Chromosomal Instability And Tumor Cell Migration ...54

3.1.1 Increased Interphase Microtubule Plus-End Assembly Rates Correlate With Migration And Invasion 54

3.1.2 Alterations In Microtubule Plus-End Assembly Rates, But Not A CIN Phenotype Affect Migration And Invasion

3.1.3 Metastasis Associated Alterations In Human Cancer Induce Increased

Microtubule Plus-End Assembly Rates. 66

3.1.4 The Microtubule Plus-End Binding Protein EB1 Is Important For Microtubule-

Dependent Signaling. 67

3.1.5 SW620 And SK-Mel-103 Cells Exhibit Elevated Levels Of Active TRIO 69

3.1.6 Inhibition Of TRIO Decreases CIN And Migration in SW620 and SK-Mel-103 Cells

3.1.7 Elevated Rac1 Activity Affects CIN And Migration In SW620 And SK-Mel-103 Cells 73

3.1.8 The Arp2/3 Complex Acts Downstream Of Rac1 During Migration And The Development Of CIN

3.1.9 Inhibition Of TRIO, Rac1 And The Arp2/3 Complex Affects Spindle Orientation In SW620 Cells 
3.1.10 Inhibition Of TRIO, Rac1 Or The Arp2/3 Complex Suppresses CIN In SW620 And SK-Mel-103 Cells 81

3.2 The Role Of p53 And p73 In Chromosomal Instability And Migration. 86

3.2.1 Loss Of TP53 And TP73 Increases Microtubule Plus-End Assembly Rates And Induces CIN

3.2.2 Abnormal Microtubule Dynamics Induced By Loss Of TP53 And TP73 Are Mediated By p21 93

3.2.3 Loss of TP53 And TP73 Causes An Invasive Phenotype In HCT116 Cells 96

3.2.4 In Vivo Analyses Of Invasiveness Of HCT116 Cells After Loss Of TP53 And TP73

4 Discussion

4.1 Increased Activity Of TRIO-Rac1-Arp2/3 Pathway As A Trigger For Migration And CIN 99

4.2 p53 And p73 Act As Regulators Of Chromosomal Stability And Cell Invasion. 105

References 109

Acknowledgement 133

Curriculum Vitae 134 


\section{List Of Figures}

Figure 1.1: The eukaryotic cell cycle 13

Figure 1.2: Regulation of the cell cycle by CDK-cyclin complexes 15

Figure 1.3: Dynamic instability of microtubules 18

Figure 1.4: Actin filament nucleation. 20

Figure 1.5: Classification of kinetochore-microtubule attachments 24

Figure 1.6: Regulation of small GTPases of the Rho family 25

Figure 1.7: Rho-family of small GTPases regulating actin remodeling. 27

Figure 1.8: Model of cell migration 29

Figure 2.1: Schematic illustration for the determination of the spindle axis angle 47

Figure 3.1: Increased interphase microtubule plus-end assembly rates correlate with a migratory and invasive phenotype. 55

Figure 3.2: Highly migratory and invasive melanoma cell lines SK-Mel-103 and SK-Mel-147 exhibit increased interphase microtubule plus-end assembly rates.

Figure 3.3: Restoration of microtubule plus-end assembly rates suppresses cell migration in $\mathrm{CRC}$ cell lines

Figure 3.4: Restoration of microtubule plus-end assembly rates suppresses the invasive phenotype in melanoma cells

Figure 3.5: Restoration of microtubule plus-end assembly rates by low dose Taxol treatment suppresses the generation of lagging chromosomes

Figure 3.6: CIN does not trigger cell migration per se

Figure 3.7: Inhibition of increased microtubule plus-end assembly rates suppresses migration and invasion

Figure 3.8: Induction of increased microtubule plus-end assembly rates triggers migration and invasion

Figure 3.9: Invasion-associated alterations in cancer correlate with increased microtubule plus-end assembly rates

Figure 3.10: Repression of EB1 reduces the migratory and CIN phenotype while having no impact on microtubule plus-end assembly rates.

Figure 3.11: Elevated levels of active TRIO in SW620 and SK-Mel-103 cells depend on increased microtubule plus-end assembly rates 
Figure 3.12: TRIO activity depends on its microtubule plus-end localization via EB1 71

Figure 3.13: Inhibition of TRIO suppresses the generation of lagging chromosomes and inhibits cell migration

Figure 3.14: Elevated levels of active Rac1 are dependent on increased microtubule plus-end assembly rates

Figure 3.15: Overexpression of a constitutively active Rac1 mutant protein induces

migration and the generation of lagging chromosomes 75

Figure 3.16: Overexpression of a dominant negative mutant protein of Rac1 reduces migration and the generation of lagging chromosomes in SW620 and SK-Mel-103 cells

Figure 3.17: Inhibition of Rac1 by its specific inhibitor decreases migration and the generation of lagging chromosomes.

Figure 3.18: Inhibition of the Arp2/3 complex decreases migration and the generation of lagging chromosomes

Figure 3.19: Analyses of spindle orientation in SW620 cells during prometaphase or metaphase

Figure 3.20: Inhibition of TRIO, Rac1 or the Arp2/3 complex suppresses CIN in SW620 cells

Figure. 3.21: CEP-FISH analyses of SW620 derived single cell clones treated with TRIO, Rac1 or Arp2/3 complex inhibitor

Figure 3.22: Inhibition of TRIO, Rac1 and Arp2/3 complex suppresses CIN in SK-Mel-103 cells

Figure 3.23: Single cell clones treated with inhibitor of TRIO, Rac1 or the Arp2/3 complex show no alterations in microtubule plus-end assembly rates but a reduce migratory phenotype

Figure 3.24: Drug removal re-induces CIN

Figure 3.25: Concomitant repression of TP53 and TP73 leads to increased microtubule plus-end assembly rates and induces the generation of lagging chromosomes

Figure 3.26: The concomitant repression of TP53 and TP73 leads to CIN 88

Figure 3.27: The CIN phenotype induced by loss of TP53 and TP73 in HCT116 cells can be suppressed by Taxol treatment.

Figure 3.28: The re-expression of TAp73 suppresses CIN in HCT116-TP53 $3^{-/} / T P 73 s h$ cells

Figure 3.29: Repression of TP73 in HCT116 cells expressing a mutant form of p53 induces CIN 
Figure 3.30: The expression of $\Delta N p 73$ increases microtubule plus-end assembly rates and induces the occurrence of lagging chromosomes

Figure 3.31: Repression of CDKN1A induces increased microtubule plus-end assembly rates and lagging chromosomes

Figure 3.32: The re-expression of $C D K N 1 A$ restores increased microtubule plus-end assembly rates observed upon repression of TP53 and TP73 in HCT116 and RKO cells 95

Figure 3.33: Loss of TP53 and TP73 induces invasion 97

Figure 3.34: Loss of TP53 and TP73 causes ulcerative invasive tumor growth in vivo 98

Figure 4.1: Model showing the microtubule-dependent hyperactivity of the TRIORac1-Arp2/3 pathway that affects both mitosis and interphase 100

Figure 4.2: Possible modes of induction of microtubule dependent actin assembly at the leading edge 102 


\section{List Of Tables}

Table 2.1 Equipment

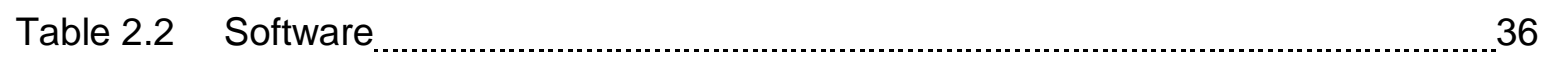

Table 2.3 Chemicals

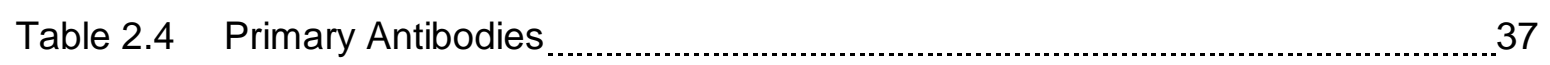

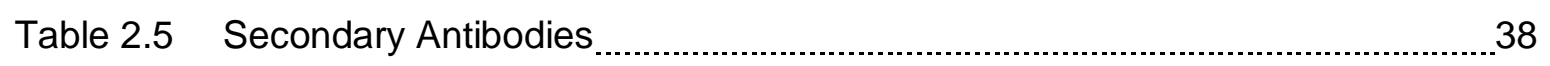

Table 2.6 Chromosome Enumeration Probes .............................................................. 39

Table 2.7 qRT-PCR Primer

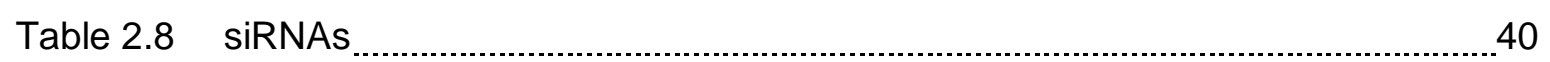

Table 2.9 Plasmids $\quad 40$

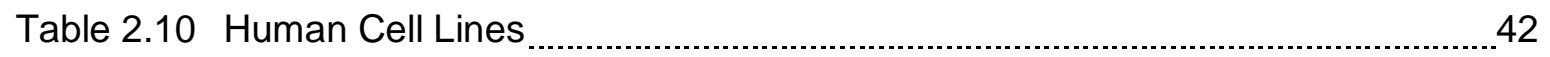

Table 2.11 Generated Human Cell Lines .................................................................... 43

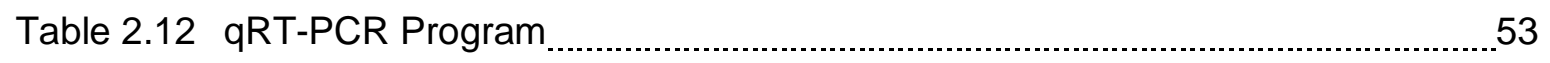

Table 3.1 Extract of deregulated genes after single or concomitant loss of TP53 and TP73 


\section{Abstract}

Aneuploidy and increased cell migration and invasion are hallmarks of aggressive human cancers. Aneuploidy derives from an increased rate of perpetual chromosome missegregation during mitosis, referred to as chromosomal instability (CIN). CIN contributes to the development of genetic heterogeneity and is thought to support rapid adaptation of cancer cells. Significantly, late tumor stages, which exhibit metastasis, are not only characterized by increased cancer cell migration and invasion, but also by high levels of CIN, both of which correlate with poor patient prognosis. Therefore, I aimed to investigate a potential link between CIN and increased cell migration and invasion in aggressive human cancer cells.

In this study, I found that CIN per se is not sufficient to trigger increased cancer cell migration and invasion. However, a hyperactive TRIO-Rac1-Arp2/3 pathway acts as a shared trigger for both, the development of CIN and cancer cell migration and invasion. Hyperactivation of TRIO, Rac1 and the Arp2/3 complex depends on increased microtubule plus-end assembly rates and on the localization of the Rac1-GEF TRIO to microtubule plus-ends via the microtubule end-binding protein EB1. In mitosis, microtubule dependent hyperactivation of the pathway causes spindle positioning defects leading to erroneous microtubule-kinetochore attachments and the generation of lagging chromosomes, which constitute a common cause for chromosome missegregation and CIN. Inhibition of TRIO, Rac1 or the Arp2/3 complex suppressed these phenotypes and prevented the development of aneuploidy in chromosomally instable colon cancer cells. In interphase, the hyperactivity of TRIO, Rac1 and the Arp2/3 complex resulted in highly enhanced cancer cell migration and invasion. Analogous to the situation in mitosis, restoration of proper microtubule dynamics in interphase suppressed the migratory and invasive phenotype in invasive colorectal cancer and melanoma cells. Thus, these results demonstrate a mechanistic link between the regulation of the actin and the microtubule cytoskeleton important for the development of CIN as well as for triggering cancer cell migration and invasion.

As one important trigger that can cause increase of microtubule plus-end assembly rates in both, mitosis and in interphase I identified a concomitant loss of the transcription factors p53 and p73. In fact, my studies suggest that p53 and p73 cooperate in maintaining chromosomal stability and suppressing cancer cell migration and invasion. 


\section{Introduction}

\subsection{The Eukaryotic Cell Cycle}

The cell cycle enables the generation of two daughter cells that are accurate copies of the parental cell. This process is divided into two main phases: mitosis and interphase. Mitosis again is subdivided into a successive series of phases: prophase, prometaphase, metaphase, anaphase and telophase, whereas interphase is subdivided into $\mathrm{G}_{1^{-}}, \mathrm{S}-$ and $\mathrm{G}_{2^{-}}$ phase (Fig. 1.1). Each phase of the cell cycle is characterized by the time-dependent activation and inactivation of enzymatic cascades, that are regulated through various protein complexes (Nigg 2001).

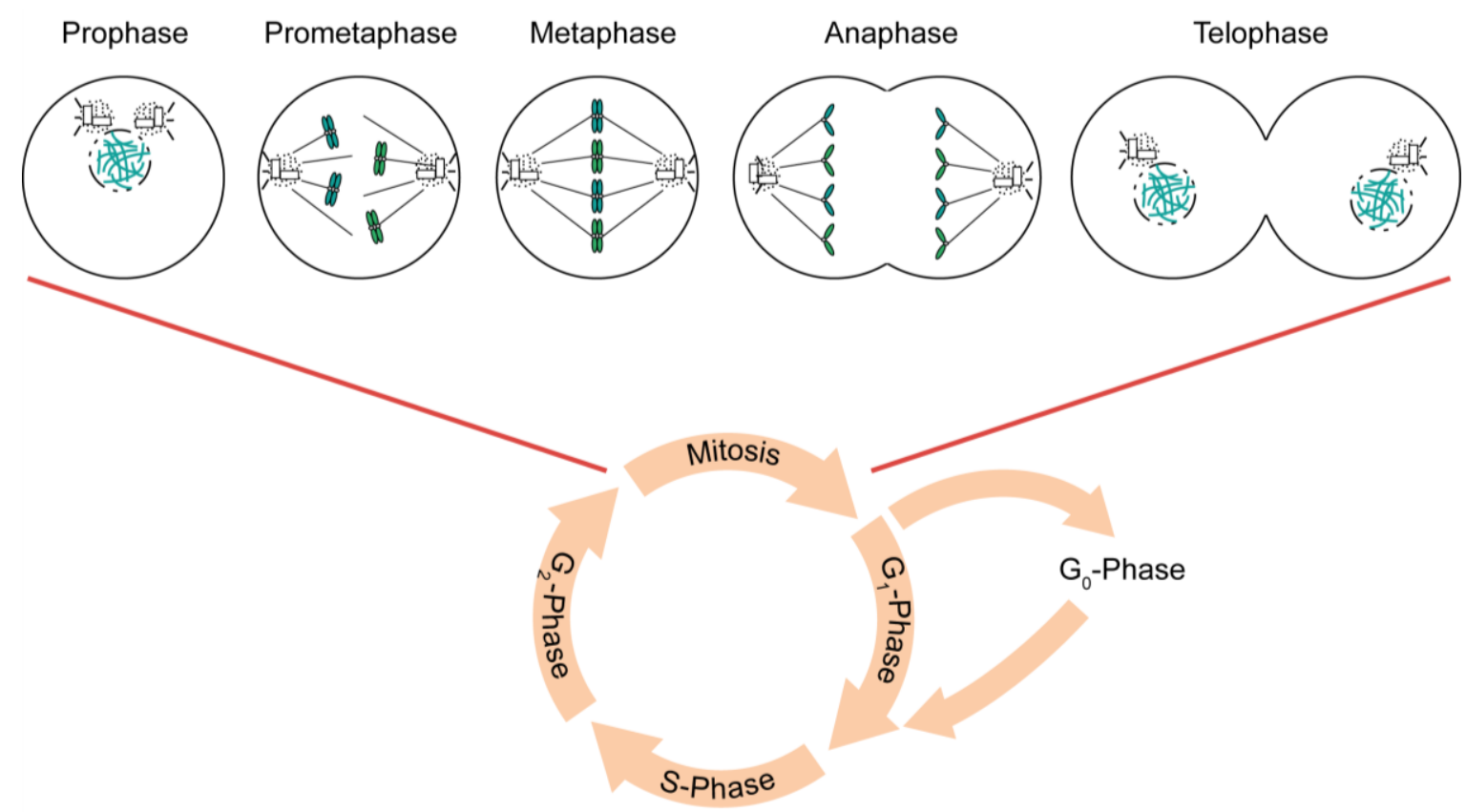

Figure 1.1: The eukaryotic cell cycle. The eukaryotic cell cycle is divided into interphase with its sub-phases $G_{1^{-}}$, $S$ - and $G_{2}$-phase and mitosis with its sub-phases prophase, prometaphase, metaphase, anaphase and telophase. During prophase, the centrosomes separate and the chromosomes starts to condense. The nuclear envelope breaks down in prometaphase and the mitotic spindle assembles. When all chromosomes are attached to spindle microtubules emanating from the opposing spindle poles and fully aligned on the cell equator, the cell is said to be in metaphase. During anaphase the chromosomes are separated and pulled towards the opposing spindle poles. A new nuclear envelope is build in telophase and the chromosomes decondense.

During $G_{1}$-phase (gap-phase 1), the cell grows in size and many genes required for cell division are switched off. In case of poor nutrient supply or during differentiation, the cell is able to exit the cell cycle to enter the resting phase $\mathrm{G}_{0}$. But upon proliferative environmental stimuli, the cell overcomes the restriction point, whereupon genes required for the transition 
into S-phase (DNA synthesis phase) are transactivated. The transition from $\mathrm{G}_{1}$ - to $\mathrm{S}$-phase needs to be tightly regulated, since the misregulation promotes oncogenesis (Bertoli et al. 2013). During S-phase, the centrosomes, which are the main microtubule organizing centers in mammals, are duplicated and the DNA is replicated. As a result, two sister chromatids exist, which are linked by the cohesin protein complex (Losada et al. 1998; Sumara et al. 2000). This complex is important for a symmetrical segregation of the sister chromatids during mitosis (Michaelis et al. 1997). The $\mathrm{G}_{2}$-phase is characterized by the accumulation and activation of enzymes, which trigger mitotic entry when reaching a critical threshold.

During the following mitosis, the replicated DNA is segregated equally onto two daughter cells. Chromosome condensation, movement of centrosomes towards the opposing spindle poles (Nigg 2001) and the formation of the mitotic spindle (Nigg \& Stearns 2011) starts in prophase. During prometaphase, the nuclear envelope breaks down and the mitotic spindle is further established. Moreover, the kinetochores assemble at the centromeric region of the sister chromatids, thereby generating binding sites for the microtubules (Cheeseman \& Desai 2008). First, the chromosomes become randomly attached by microtubules emanating from the spindle poles in a process termed "search and capture" (Kirschner \& Mitchison 1986). Then they are aligned at the metaphase plate in an accurate manner. Once all chromosomes are properly attached to spindle microtubules and fully aligned, the cell is said to be in metaphase. During anaphase A, cohesin protein complexes, which link the sister chromatids, are cleaved by the enzyme separase (Nakajima et al. 2007), the sister chromatids are separated and move towards the spindle poles due to microtubule shortening, while in anaphase B the two spindle poles themselves move apart (Rieder \& Salmon 1994). In telophase, the chromatids reach the spindle poles thereby forming the new daughter nuclei. The separation of the daughter cells is completed by cytokinesis.

\subsection{Cell Cycle Regulation And Checkpoints}

The cell cycle needs to be highly regulated to avoid the formation of abnormal daughter cells. The timely ordered progression of cells through the cell cycle is mediated by the oscillating activation and inactivation of cyclin-dependent kinases (CDKs) (Arellano \& Moreno 1997). The catalytic activity of CDKs requires the binding of regulatory subunits known as cyclins (Malumbres \& Barbacid 2005). The cell cycle dependent synthesis and degradation of cyclins regulate CDK activity in a timely manner (Malumbres \& Barbacid 2009). However, binding of cyclins to CDKs is not sufficient to fully activate the complex. CDK activity is also regulated by activating and inhibitory phosphorylations and dephosphorylations (Coleman \& Dunphy 1994; Lolli \& Johnson 2005). The kinases Wee1 and Myt1 inhibit CDK-cyclin complexes by phosphorylation, whereas the phosphatase Cdc25 removes these inhibitory 
phosphorylation (Malumbres \& Barbacid 2005). Furthermore, an activating phosphorylation through the CDK activating kinase (CAK) is required (Lolli \& Johnson 2005). Specific CDKcyclin complexes control cell cycle progression: D-type cyclins bind to CDK4 and CDK6 during $\mathrm{G}_{1}$-phase, E-type cyclins preferentially bind to CDK2 at the $\mathrm{G}_{1}$-S-transition, CDK2cyclin $A$ is active during $S$-phase and CDK1-cyclin $A$ and CDK1-cyclin $B$ at the transition to mitosis as well as during mitosis (Malumbres \& Barbacid 2009) (Fig. 1.2).

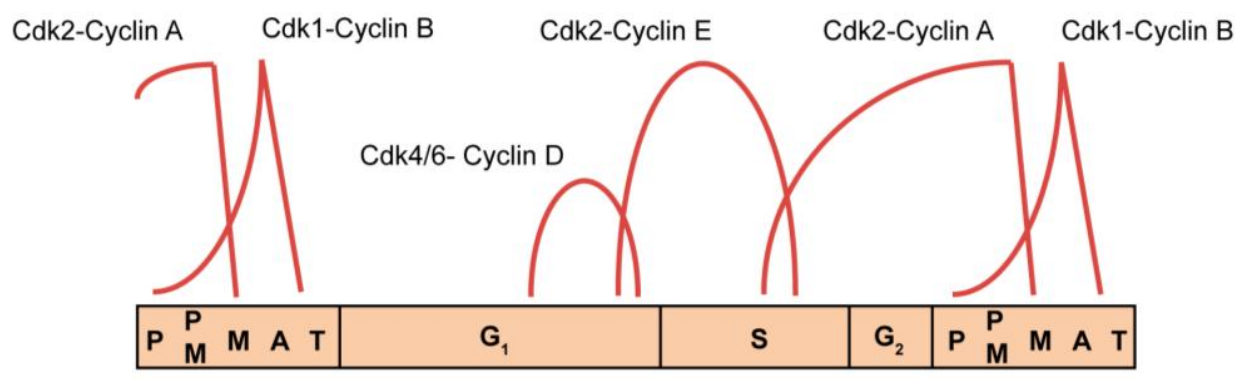

Figure 1.2: Regulation of the cell cycle by CDK-cyclin complexes. In early mitosis, Cdk1 and Cdk2 bound to cyclins $A$ and $B$ are highly active. The $G_{1}-S$-transition is mediated by Cdk4/6-Cyclin $D$ and Cdk2-Cyclin E activity, which also lead to the synthesis of proteins needed for DNA replication in S-phase. Cdk2-Cyclin A is active during S-phase until early mitosis. P: prophase, PM: prometaphase, M: metaphase, A: anaphase, T: telophase. Modified from Pollard \& Earnshaw 2007.

Signaling pathways control the progression of the cell during the cell cycle and regulate the transition between the different phases by modulating CDK activity. For instance, the DNAdamage checkpoint is activated upon diverse alterations in the DNA caused by environmental or endogenous stress (Bartek et al. 2004). Central checkpoint proteins are ATM (ataxia telangiectasia mutated) and ATR (ATM-Rad3-related) and their effectors Chk1 (Liu et al. 2000), Chk2, BRCA1 (Cortez 1999) and p53 (Banin et al. 1998; Matsuoka et al. 1998). DNA double strand breaks during $G_{1}$-phase activate ATM and Chk2, which leads to the stabilization of the transcription factor p53 (Banin et al. 1998). This in turn results in the induction of the cyclin-dependent kinase inhibitor p21, which binds to CDK-cyclin complexes, thereby blocking cell cycle progression into S-phase (Harper et al. 1993; Harper et al. 1995). During $G_{2}$-phase, the activation of Chk1 by ATR leads to a phoshorylation and thereby inhibition of Cdc25. As a consequence, Cdc25 is not able to activate CDK1-cyclin B, thus preventing mitotic entry in the presence of damaged DNA (Sanchez 1997).

During mitosis, the spindle assembly checkpoint (SAC) ensures genomic stability by delaying chromosome segregation until all kinetochores are properly attached to spindle microtubules. During prometaphase, the mitotic checkpoint complex (MCC), consisting of the proteins BubR1, Bub3, Cdc20 and Mad2, assembles at unattached kinetochores (Lara-Gonzalez et al. 2012). Due to this complex formation, Cdc20 is not able to activate the E3 ubiquitin ligase 
anaphase-promoting complex/cyclosome (APC/C) (Musacchio \& Salmon 2007). When all chromosomes are bi-oriented and aligned at the metaphase plate, the SAC is satisfied and the generation of the MCC declines. Cdc20 is released from the complex and can activate $\mathrm{APC} / \mathrm{C}$, which leads to the ubiquitylation of cyclin $\mathrm{B}$ and securin and their subsequent proteasomal degradation. Proteolysis of securin results in the release of the protease separase. In turn, separase cleaves the cohesin complexes that are responsible for sister chromatid cohesion, thereby enabling chromosome segregation. On the other hand APC/Cmediated proteasomal degradation of cyclin B inactivates the cyclin-dependent kinase 1 (CDK1), thereby promoting mitotic exit (Peters 2006).

\subsection{The p53 Tumor Suppressor Family}

The TP53 gene encoding for p53 is among the most frequently altered tumor suppressor genes in human cancers. About $50 \%$ of human cancers harbor mutations in this gene, leading to an overproduction of mutant protein in high concentrations. p53 is a sequence specific transcription factor, which is involved in regulating the expression of genes controlling cell cycle arrest and apoptosis. p53 is activated upon genotoxic damage or metabolic stress and plays a central role in $\mathrm{G}_{1}$ and DNA damage checkpoints. Upon DNA damage p53 induces DNA repair proteins with subsequent cell cycle arrest until the damaged DNA is repaired. In case of irreparable DNA damage, p53 is also able to induce programmed cell death, thus preventing the proliferation of cells with highly damaged DNA (Amundson et al. 1998).

The p53 family members p73 and p63 were found to have a high structural and functional similarity to p53 (Yang et al. 1998; Kaghad et al. 1997). They share the hallmark features of p53's structure: an amino-terminal transactivation domain, a highly conserved DNA-binding domain and a carboxy-terminal oligomerization domain (Levrero et al. 2000). Therefore, they might be able to fulfill redundant functions of p53. Indeed, it was shown, that TA isoforms of p73 and p63 can bind to p53-responsive promotor elements of well-known p53 target genes like $C D K N 1 A, B A X$ or MDM2 (Yang \& McKeon 2000). In addition, so-called $\triangle \mathrm{N}$ isoforms, which are truncated at their $\mathrm{N}$-terminus, have been shown to act antagonistically to p53 as well as to TAp73 and TAp63 (Yang et al. 1998; Grob et al. 2001). These $\Delta \mathrm{N}$ isoforms are still capable of DNA-binding and thus might compete with p53, TAp73 and TAp63 for DNAbinding sites. Because of the conserved oligomerization domain, $\Delta \mathrm{N}$ proteins are also able to bind to p53, TAp73 and TAp63 leading to sequestration of the proteins and formation of inactive hetero-oligomers (Yang \& McKeon 2000; Grob et al. 2001). Furthermore, $\Delta N p 73$ is highly overexpressed in various tumors (Concin et al. 2004) including ovarian and skin cancer. The overexpression is associated with poor patient prognosis and decreased survival 
(Concin et al. 2005). $\triangle N p 73$ expression can also be found in metastases of skin cancer patients (Tuve et al. 2004). Here, $\Delta$ Np73 induces epithelial-mesenchymal-transition (EMT), with loss of $\mathrm{E}$-cadherin and induction of $\mathrm{N}$-cadherin (Engelmann et al. 2014).

A frequent lesion in colorectal cancer is a mutation in TP53 (Muller \& Vousden 2013), which causes loss of lost wild-type function. Mutant p53 protein exerts a dominant-negative regulation towards the remaining wild-type p53 (Petitjean et al. 2006). Mutations can be found throughout the TP53 gene but they cluster within the DNA-binding domain. Several hot spot mutations were identified, including occurrence at R175, G245, R248 and R273. These mutations affect folding of the protein and also alter the DNA-binding ability of p53. Mutant p53 still exerts DNA-binding activity, nevertheless, the binding to p53 responsive elements is impaired (Muller \& Vousden 2013; Thukral et al. 1995). However, mutant p53 can also bind to unique DNA-elements, which indicates a gain of function and gives mutant p53 the function of an oncogenic transcription factor (Kim \& Deppert 2004; Muller \& Vousden 2013). Transcriptional functions of mutant p53 are also achieved via a direct interaction with other transcription factors to prevent or even enhance their function. An inhibitory interaction can be found between mutant p53 and p73 as well as p63. Thereby, mutant p53 binds to and inhibits the TA isoforms of p73 and p63 (Gaiddon et al. 2001).

\section{4 p21 As Mediator Of p53 Tumor Suppressor Activity}

p21 is a member of the Cip and Kip family of CDK inhibitors, which includes p21, p27 and p57. The gene encoding for p21 (CDKN1A) is activated upon DNA-damage by p53 and p21 protein mediates a cell cycle arrest (Deng et al. 1995) by inhibiting CDK2, CDK4 and CDK6 (Harper et al. 1995). CDK2 activity is required for phosphorylation of the Rb (retinoblastoma) protein. Upon phosphorylation, $\mathrm{Rb}$ is released from a complex consisting of $\mathrm{Rb}$ and the transcription factor E2F. This complex disruption leads to E2F-dependent gene expression, which is needed to overcome the restriction point. p21 is frequently deregulated in cancers and is able to promote proliferation and oncogenesis (Rufini et al. 2011). This deregulated expression is often associated with a loss of function of transcriptional regulators such as p53 (Abbas \& Dutta 2009). Reduced CDKN1A expression is detected in colorectal, cervical, head and neck as well as small-cell lung cancers (Ogino et al. 2010). Ahead from p21's role in the DNA damage checkpoint, p21 deficiency was shown to induce mitotic defects (Kreis et al., 2014). These defects include prolonged metaphase and anaphase as well as erroneous chromosome segregation and cytokinesis, which promote genomic instability. However, the underlying mechanisms are unclear. 


\subsection{The Cellular Cytoskeleton}

\subsubsection{The Microtubule Cytoskeleton}

The microtubule cytoskeleton is important for a large number of cellular functions. During mitosis the most prominent function is the assembly of the mitotic spindle and the precise chromosome segregation (Wittmann et al. 2001), whereas during interphase microtubules participate in organelle positioning, intracellular transport as well as maintaining cell shape and facilitating cellular motility (Goode et al. 2000). Microtubules are assembled of $\alpha$ - and $\beta$ tubulin heterodimers, which form a protofilament. 13 protofilaments form the hollow tube of microtubules. Microtubules exhibit a certain polarity with $\alpha$-tubulin exposed at the minus-end and $\beta$-tubulin exposed at the plus-end. Assembly and disassembly of tubulin heterodimers occurs solely at their ends. Each end switches between growth and shrinkage, whereby dissociation of tubulin mainly occurs at the minus-end and association at the plus-end. Thereby, microtubules grow and shrink at a steady state, a process termed 'treadmilling' (Margolis \& Wilson 1998; Grego et al. 2001).
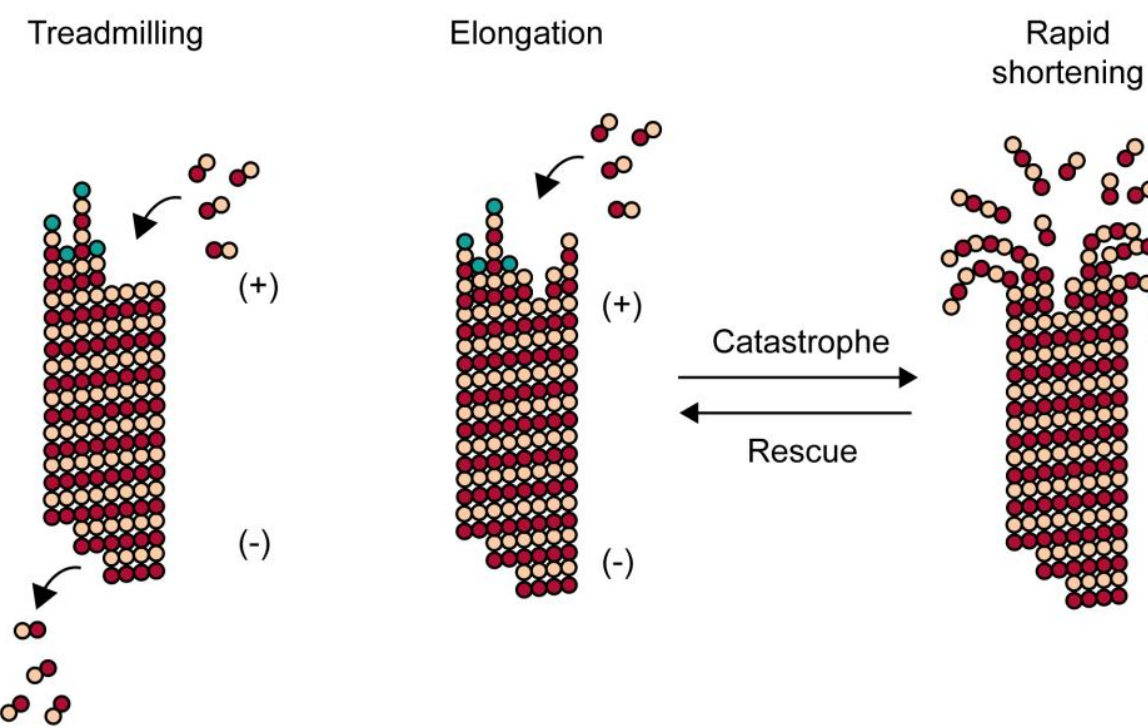

Figure 1.3: Dynamic instability of microtubules. Microtubules are assembled by $\alpha-/ \beta$ - tubulin heterodimers that are incorporated at the microtubule plus-end. The assembly at the plus-end and disassembly of heterodimers at the minus-end, without changes in length is termed treadmilling. However, microtubules are characterized by dynamic instability. They undergo transition to rapid shortening, which is termed catastrophe. This process can be terminated by a rescue event whereupon the microtubule grows again. Modified from Pollard \& Earnshaw 2007.

Microtubule growth is regulated by the incorporation of the tubulin heterodimer into the microtubule end and the hydrolysis of $\beta$-tubulin bound GTP. GTP hydrolysis occurs with a delay, resulting in a so-called GTP-cap at the microtubule tip. The GTP-cap stabilizes the microtubule and loss of the cap leads to a catastrophe event with microtubule 
depolymerization and shrinkage. The switch between growth and shrinkage is known as catastrophe, while the switch from shrinkage to growth is termed 'rescue'. Microtubule disassembly can be rescued by the presence of GTP-islands on the surface of the microtubule lattice, which mimic the GTP-cap (Desai \& Mitchison 1997; Akhmanova \& Steinmetz 2015) (Fig. 1.3).

The dynamicity of microtubules is regulated by microtubule associated proteins (MAPs) and their sub-class of microtubule plus-end tracking proteins (+TIPs) (Schuyler \& Pellman 2001). Microtubule polymerases, such as ch-TOG (colonic and hepatic tumour-overexpressed gene), bind to microtubule plus-ends and promote the incorporation of tubulin heterodimers into the growing plus-end (Gard \& Kirschner 1987; Brouhard et al. 2008). Microtubule depolymerases, such as MCAK (mitotic centromere-associated kinesin), regulate microtubule disassembly by removing heterodimers from the microtubule minus-ends (Hunter et al. 2003; Burns et al. 2014). Indirectly, also the end-binding proteins (EBs) of + TIPs can regulate microtubule polymerization rates. Hundreds of EB proteins like EB1 can bind to a growing microtubule tip (Vaughan 2005), thereby serving as a binding platform for other proteins like CLIP-170 (cytoplasmic linker protein) (Lansbergen et al. 2004), CLASP1 and CLASP2 (cytoplasmic linker protein associated proteins 1/2) (Mimori-Kiyosue et al. 2005) and $\mathrm{p} 150^{\text {Glued }}$ (Ligon et al. 2003; Watson \& Stephens 2006). These proteins appear to promote rescue events or suppress catastrophe events (Akhmanova \& Steinmetz 2015).

\subsubsection{The Actin Cytoskeleton}

Actin plays an important role in several cellular processes like cell motility, establishment and maintenance of cell shape, cytokinesis and muscle contraction. Actin filaments are assembled of actin polymers and a variety of actin-binding proteins (ABPs), which include filament crosslinkers, motor proteins as well as nucleation and elongation factors (Lee \& Dominguez 2010; Mullins \& Hansen 2013).

In the cell, actin is present in a monomeric, globular (G-actin) or polymeric, filamentous ( $F$ actin) form. G-actin is able to bind ATP, which is hydrolyzed to ADP, when actin is incorporated into a growing filament. F-actin forms a double-stranded helix and exhibits a certain polarity with a barbed (+) end and a pointed (-) end. Spontaneous addition of ATPbound G-actin at the barbed $(+)$ end leads to growing of the actin filament, whereas depolymerization of F-actin occurs at the pointed (-) end by dissociation of ADP-bound G-actin (Lee \& Dominguez 2010) (Fig. 1.4). This so-called actin filament treadmilling is regulated by ABPs. Among them, ADF/cofilin and profilin accelerate the dissociation of G-actin from the pointed end and the incorporation to the barbed end, respectively. ADF/cofilin and profilin are ABPs that bind to G-actin, thereby controlling the pool of 
unpolymerized actin in the cell (Lee \& Dominguez 2010). During actin polymerization, an actin/profilin complex is guided to the barbed end of a filament and incorporated into the filament by the filament elongation factor Eva/VASP (Lee \& Dominguez 2010).
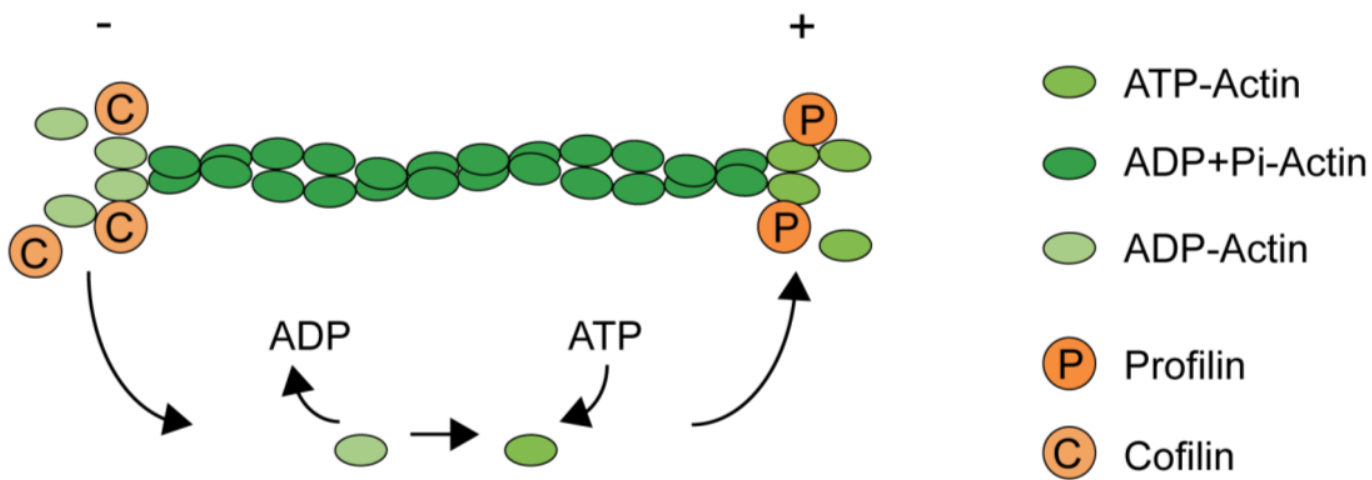

Figure 1.4: Actin filament nucleation. Actin filaments form a double-helix and exhibit a barbed $(+)$ and a pointed (-) end. Association of ATP-bound actin monomers mainly occurs at the barbed end, whereas the pointed end is mainly characterized by dissociation of ADP-bound actin monomers. Actin binding proteins (ABPs) regulate actin treadmilling. Profilin promotes incorporation of actin monomers at the barbed end, whereas cofilin promotes actin disassembly from the pointed end. Modified from Lee \& Dominguez 2010

Actin filaments can exhibit different types of organization: branched and crosslinked networks, parallel bundles and anti-parallel contractile structures (Blanchoin et al. 2014). The Arp2/3 complex (actin related protein $2 / 3$ ) is responsible for branching of a pre-existing actin filament. This complex is activated by the Nucleation Promoting Factors (NPF) WASP (Wiskott-Aldrich syndrome protein) or WAVE (WASP-family verprolin-homologous protein), which interact with Arp2/3 and actin monomers to create a nucleation core at the side of a mother filament (Lee \& Dominguez 2010; Achard et al. 2010). The presence of capping protein (CP) limits the growth of the filament by binding to the barbed end (Akin \& Mullins 2008). Surprisingly, this CP-mediated termination of filament elongation promotes actin network assembly and cell motility (Achard et al. 2010). Whereas the Arp2/3 complex is involved in the initiation of actin assembly as well as in the organization of the actin network, there are also proteins, which solely connect actin filaments without modulating their assembly, e.g. the long crosslinkers alpha-actinin and filamin, and the short crosslinkers fimbrin and fascin. Depending on the crosslinking proteins, actin is packed into tight parallel or antiparallel bundles or a filament network is built. Branched and crosslinked filaments make up the lamellipodium, whereas aligned bundles are the basis for filopodia and stress fiber formation (Blanchoin et al. 2014). Alterations in the actin cytoskeleton are associated with cancer metastasis and invasion, since the invasion process is characterized by the formation of lamellipodia, filopodia and invadopodia (Yamaguchi \& Condeelis 2007). 
During mitosis, the actin cytoskeleton is subjected to dynamic rearrangements. Actin filaments localize to the cortical plasma membrane, to retraction fibers and also to the contractile ring during late stages of mitosis and cytokinesis (Mitsushima et al. 2010). The cortical actin influences spindle positioning, thereby defining the cell's division axis (Théry et al. 2005; Toyoshima \& Nishida 2007). Furthermore, an amorphous cluster known as 'actin cloud' was recently described to be present during mitosis (Mitsushima et al. 2010), which interacts with astral microtubules, influencing mitotic spindle alignment (Fink et al. 2011). The actin cloud forms at the cell cortex early during mitosis, persists until late anaphase and was shown to be dependent on the activity of the Arp2/3 complex (Mitsushima et al. 2010).

\subsection{Mitotic Spindle Assembly}

The dynamic properties of microtubules along with many proteins that modulate microtubule organization and stability are required to set up a bipolar spindle during mitosis to separate the sister chromatids (Gadde \& Heald 2004). Three sub-populations of microtubules exist within a mitotic spindle: kinetochore microtubules connect the chromosomes to spindle poles, interpolar microtubules form an overlapping, antiparallel network and astral microtubules interact with the cell cortex (Wittmann et al. 2001; Gadde \& Heald 2004). The first step towards a bipolar spindle is the separation of the centrosomes, which is mediated by motor proteins (Wittmann et al. 2001). Motor proteins can be classified into plus-end directed motors, known as kinesins, and minus-end directed motors, known as dyneins. Among them, the kinesin Eg5 plays an important role during centrosome separation (Whitehead \& Rattner 1998; Tanenbaum et al. 2008). Due to the antiparallel sliding activity of Eg5, the centrosomes are pushed to opposing sides (Tanenbaum \& Medema 2010). A second important player is the minus-end directed motor dynein (Tanenbaum \& Medema 2010). Dynein localizes to astral microtubules or to the cell cortex (Kardon \& Vale 2009). The cortical dynein is able to pull on astral microtubules, thereby positioning the mitotic spindle (Grill et al. 2003). The main function of the mitotic spindle is the alignment and the segregation of the chromosomes. For this, microtubules are attached to kinetochores, which involves several kinetochore and microtubule associated proteins. Chromosome alignment and segregation requires the function of different kinesins, including chromokinesins.

Precise spindle positioning is an important process, since it provides the axis of the following cell division. Usually, the axis of cell division is oriented along the planar axis, thereby ensuring the attachment of the newly formed daughter cells to the substratum (Toyoshima \& Nishida 2007). The polarization depends on external factors such as the extracellular matrix or neighboring cells (Fink et al. 2011). Astral microtubules interact with cortical proteins via +TIPs, including EB1, APC (adenomatous poliposis coli) and dynein (Schuyler \& Pellman 
2001). The dynein-dynactin complex binds to NuMA (Nuclear mitotic apparatus protein), that is linked to the cell cortex via LGN (GPSM2, G-protein-signaling modulator 2). The minusend directed motor activity of dynein-dynactin then provides a pulling force on astral microtubules (Bergstralh \& St Johnston 2014).

Furthermore, the actin cytoskeleton is necessary during spindle positioning, since the disruption of actin was shown to induce spindle misorientation (Toyoshima \& Nishida 2007). The actin cytoskeleton provides information about the cell's shape and adhesion. The distribution of adhesions in interphase cells was described to determine the mitotic spindle orientation (Théry et al. 2005). Thereby, the actin cytoskeleton is associated with membrane ruffles in interphase, which contain microtubule stabilizing proteins such as APC (EtienneManneville \& Hall 2003) and motor proteins such as dynein (Busson et al. 1998). These proteins remain located in this area during cell division and promote the localization of the spindle poles (Dujardin et al. 2003). Additionally, forces generated by retraction fibers lead to Arp2/3-based subcortical actin structures in mitotic cells, which exert pulling forces on the mitotic spindle (Fink et al. 2011). These actin structures are possibly coupled to microtubules, thereby influencing spindle positioning, but its function is largely unknown.

\subsection{Chromosomal Instability And Aneuploidy}

Accurate progression of mitosis is pivotal to ensure a correct euploid karyotype. However, many human diseases including cancer and neurodegenerative diseases are characterized by aneuploidy. A common cause for aneuploidy in human cancer cells is chromosomal instability (CIN) (Lengauer et al. 1997). One can distinguish between structural chromosomal instability (S-CIN) that describes the susceptibility to structural rearrangements including translocations, deletions, inversions and duplications of chromosomal parts (Ricke et al. 2008; Thompson et al. 2010) and whole chromosomal instability (W-CIN), which is defined as the perpetual gain or loss of whole chromosomes during mitosis. In a typical aneuploid cancer cell, one chromosome in every one to five cell divisions becomes missegregated (Lengauer et al. 1997; Thompson \& Compton 2008). It is thought that these low missegregation rates allow the acquirement of new cancer phenotypes and the adaptation to the environment (Thompson et al. 2010). In contrast, high rates of chromosome missegregation induced by a highly deregulated mitosis are lethal (Kops et al. 2004). Furthermore, CIN and aneuploidy is of disadvantage for tumor growth, probably caused by metabolic changes and proteotoxic stress (Torres et al. 2007; Sheltzer \& Amon 2011; Ertych et al. 2014). 
The molecular mechanisms causing CIN in human cancer cells are not well understood, but could involve abnormalities during interphase as well as various alterations in mitotic progression (Orr \& Compton 2013).

A controversially discussed mechanism is an impaired spindle assembly checkpoint (SAC). The SAC senses improper kinetochore-microtubule attachments and maintains genomic stability by delaying the metaphase-to-anaphase-transition until all chromosomes are amphitelically attached (Fig. 1.5). A defective SAC leads to a premature anaphase onset in the presence of faulty kinetochore-microtubule attachments, resulting in chromosome missegregation. However, in human cancer cells exhibiting CIN, a weakened SAC due to mutations in SAC related genes is rarely found (Tighe et al. 2001; Barber et al. 2008) and the complete loss of SAC function was even shown to be lethal (Kops et al. 2004).

Chromosome missegregation can also result from the presence of supernumerary centrosomes, which occur from an aberrant cytokinesis or from defects in centrosome biogenesis or centrosome amplification during interphase. In principal, cells containing more than two centrosomes can build up a multi-polar spindle resulting in massive chromosome missegregation. However, this was shown to be unviable for progenies arising from multipolar cell divisions (Ganem et al. 2009). Instead, supernumerary centrosomes often cluster to form a pseudo bi-polar spindle (Brinkley 2001). But still, the transient occurrence of a multi-polar spindle promotes transient spindle geometry defects, erroneous kinetochoremicrotubule attachments and lagging chromosomes (Ganem et al. 2009), leading to chromosome missegregation.

Lagging chromosomes are widely recognized as a cause for chromosomal instability and arise from merotelic kinetochore attachments (Fig. 1.5a). In this case, one kinetochore is concomitantly attached to spindle microtubules emanating from the two opposing spindle poles (Cimini et al. 2001; Cimini et al. 2002; Thompson \& Compton 2008). Normally, sister chromatids are attached to opposing poles of the spindle, known as amphitelic attachments. During chromosome alignment, one kinetochore becomes attached first and orients towards the spindle pole (Rieder \& Salmon 1998). This monotelically attached chromosome moves poleward until microtubules bind to the unattached kinetochore, resulting in an amphitelic attachment and chromosome bi-orientation (Rieder \& Salmon 1998; Cimini et al. 2002). But errors in kinetochore attachment can occur, including syntelic attachments, where both sister chromatids are attached to spindle microtubules emanating from the same spindle pole. In addition, merotelic attachments are often detectable in cancer cells (Fig. 1.5a). Merotelic attachments support chromosome alignment and the establishment of the metaphase plate, but these errors are not detected by the SAC and lead to a chromosome remaining near the spindle equator (Cimini et al. 2001) (Fig. 1.5b). During the following cytokinesis, the lagging chromosome is distributed onto one of the daughter cells by chance. 
a

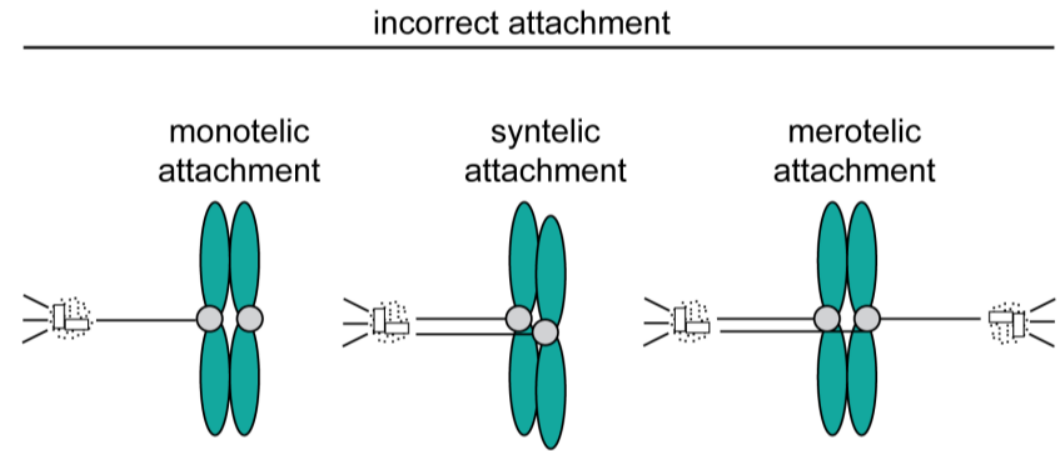

b

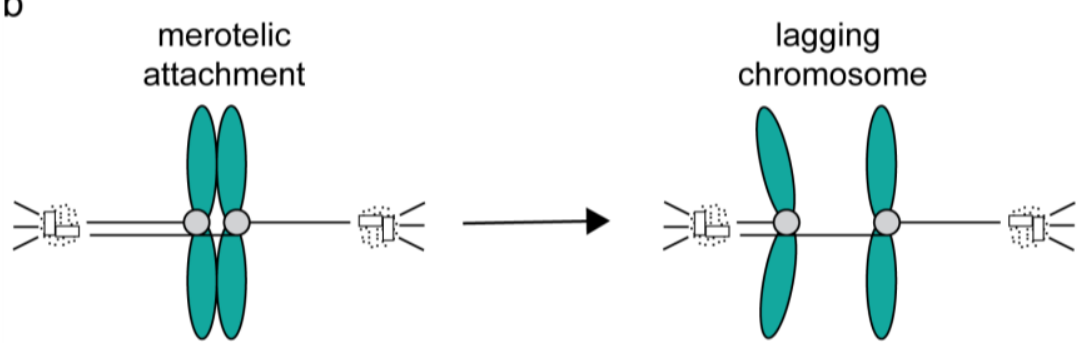

Figure 1.5: Classification of kinetochore-microtubule attachments. (a) Amphitelic attachments describe the state in which both sister kinetochores are attached to spindle microtubules emanating from the opposing spindle poles. In case of monotelic attachments, only one kinetochore is attached to microtubules emanating from one spindle pole, while syntelic attachments refer to the attachment of both sister kinetochores to spindle microtubules emanating from the same spindle pole. Lagging chromosomes arise from merotelic attachments, which describes the attachment of sister kinetochores to spindle microtubules emanating from the opposing spindle poles, whereby one kinetochore is also attached to microtubules from both spindle poles. (b) Merotelic attachments lead to the generation of lagging chromosomes during anaphase. The chromatid is randomly segregated onto the daughter cells.

During progression through mitosis, erroneous attachments can be corrected. Monotelic attachments will be sensed by the SAC (Rieder et al. 1995), whereas syntelic attachments generate low tension between sister kinetochores (Pinsky \& Biggins 2005; Nezi \& Musacchio 2009). Like syntelic attachments, merotelic attachments can be resolved by an error correction machinery involving the Aurora B kinase (Cimini et al. 2003; Knowlton et al. 2006; Holland et al. 2009). Aurora B is localized to the inner centromere and phosphorylates outer kinetochore components like Ndc80 (Cheeseman et al. 2006; DeLuca et al. 2006), Dam1 (Cheeseman et al. 2002), Ska1 (Chan et al. 2012; Schmidt et al. 2012) and MCAK (Gorbsky 2004), thereby destabilizing kinetochore-microtubule attachments. However, increased rates of the generation of erroneous microtubule-kinetochore attachments might overload the error correction machinery leading to the persistence of lagging chromosomes.

Increased microtubule plus-end assembly rates constitute a novel route to chromosomal instability, recently described by our lab (Ertych et al. 2014). MIN/MSI and CIN cell lines were analyzed regarding their microtubule plus-end assembly rates during mitosis. These analyses revealed elevated rates in all analyzed CIN cell lines. It is assumed, that increased 
microtubule plus-end assembly rates lead to transient spindle geometry defects, which result in hyper-stable kinetochore-microtubule attachments, the occurrence of lagging chromosomes and CIN. In turn, restoration of proper microtubule assembly rates by genetic means or chemicals suppresses the CIN phenotype. Vice versa, an increase of microtubule plus-end assembly rates by genetic means also induced CIN and aneuploidy (Ertych et al. 2014).

Furthermore also abnormalities in interphase might contribute to whole CIN. In fact, replication stress during S-phase was shown to affect chromosome segregation but this observation is still debated (Bakhoum et al. 2014).

\subsection{The Ras Homologous (Rho) Family Of Small GTPases}

Small GTPases of the Rho family are intracellular signaling molecules, best known for their role in regulating the actin cytoskeleton, in vesicle trafficking, cell cycle regulation and transcriptional reprogramming (Cain \& Ridley 2009).

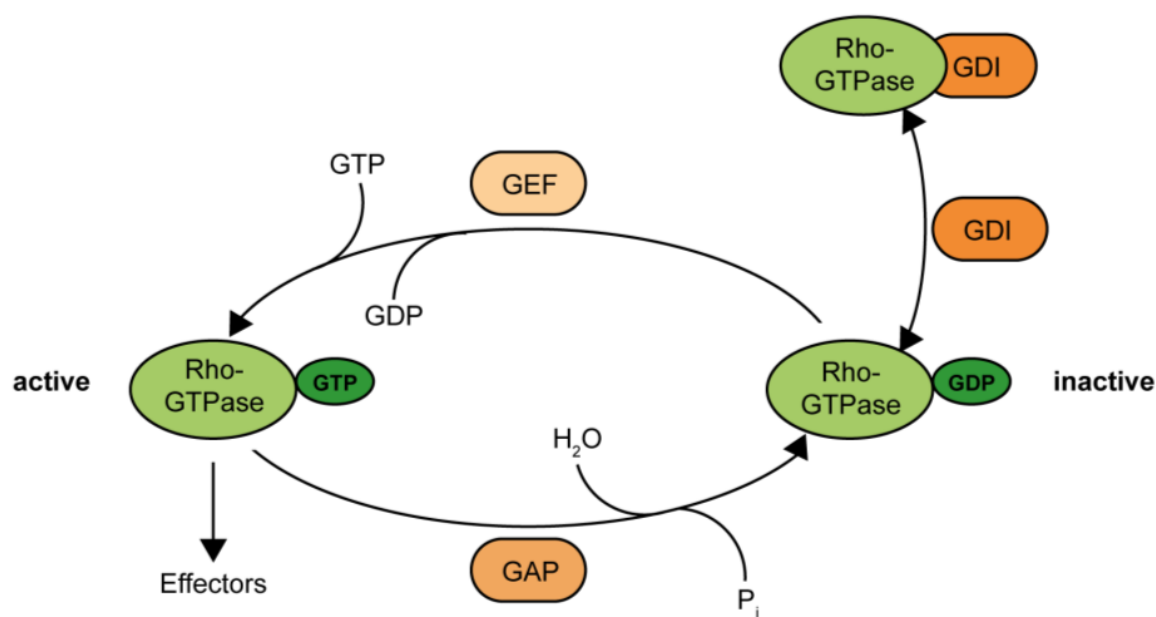

Fig. 1.6: Regulation of small GTPases of the Rho family. Inactive Rho-GTPases are activated by the exchange of GDP for GTP mediated by GEFs. GAPs inactivate Rho-GTPases by catalyzing the hydrolysis of GTP to GDP. GDIs bind inactive Rho-GTPases in the cytosol and prevent the nucleotide exchange. Modified from Lawson \& Burridge 2014

Rho GTPases exist in either an inactive GDP-bound form or in an active GTP-bound form (Fife et al. 2014). Three different classes of regulatory molecules modulate the activity of the Rho-family small GTPases: guanine nucleotide exchange factors (GEFs), GTPase-activating Proteins (GAPs) and guanine nucleotide dissociation inhibitors (GDIs) (Lawson \& Burridge 2014). While GEFs catalyze the exchange of bound GDP for GTP, GAPs stimulate the intrinsic GTPase activity, turning the Rho-family proteins into an inactive state. GDIs maintain 
a cytosolic pool of inactive Rho-family small GTPases. If required, they can be transported to cell membranes, where the nucleotide exchange takes place (Leung et al. 1995; Zheng 2001; Rossman et al. 2005; Bos et al. 2007) (Fig. 1.6).

Among Rho GTPases, the best-studied members are RhoA, Rac1 and Cdc42 and their role during cell motility (Burridge \& Wennerberg 2004). For a long time, RhoA was thought to be inhibitory for cell migration, since it promotes stress fiber formation and strong adherence mediated by focal adhesions (Lawson \& Burridge 2014). But RhoA was also found to be active at the leading edge of migrating cells, where it is responsible for membrane ruffling and the formation of lamellipodia (O'Connor et al. 2000; Machacek et al. 2009; El-sibai et al. 2009). The switch between stress fiber formation and lamellipodia formation is not well understood but might be mediated by two different GEFs, whereby one potentially activates Rho at the leading edge and the other one at the rear of the cell (Sadok \& Marshall 2014). The two homologs RhoA and RhoC were described to have different roles in cell migration since they act through different targets (Vega et al. 2011). While RhoC inhibits the development of lamellipodia through the formin FMNL3, RhoA promotes tail retraction via its effectors mDia (mammalian homolog of Drosophila diaphanous) and ROCK (Rho-associated kinase) (Narumiya et al. 2009; Vega et al. 2011). Both are well described regarding their roles in stress fiber formation. The activation of $\mathrm{mDia}$ and $\mathrm{ROCK}$ constitutes the trigger for the assembly of actomyosin filaments (Hall 2012). Here, ROCK phosphorylates and inactivates the myosin phosphatase and activates myosin light chain, resulting in an enhancement of actomyosin contractility (Kimura et al. 1996). Subsequently, contraction leads to bundling of actin filaments and clustering of integrins into focal adhesions (Narumiya et al. 2009). Actin polymerization itself is then stimulated by $\mathrm{mDia}$.

During cell motility, RhoA acts in concert with Rac1 and Cdc42 (El-sibai et al. 2009; O'Connor et al. 2012). In contrast to RhoA, Rac1 and Cdc42 promote Arp2/3-based actin polymerization and branching in the lamellipodium by activating the WAVE or WASP protein complexes (Bid et al. 2013; Blanchoin et al. 2014). Effectors of Rac1 and Cdc42 are, among others, p21-activated kinase (PAK), WAVE/WASP, IQGAP1 (IQ motif containing GTPase activating protein 1) and IQGAP2 (IQ motif containing GTPase activating protein 2) (Kuroda et al. 1999). Rac1 activates the p21-activated kinases PAK1, PAK2 and PAK3, which themselves activate the actin-binding LIM kinases LIMK1 and LIMK2. These in turn phosphorylate the actin binding protein cofilin, thereby inactivating its activity of converting $\mathrm{F}$ actin into G-actin and allowing actin growth (Ridley 2006; Bid et al. 2013). Rac1 and Cdc42 were also described to recruit $\mathrm{mDia}$ to RhoA, thus facilitating its activity during lamellae formation (Kurokawa \& Matsuda 2005). 


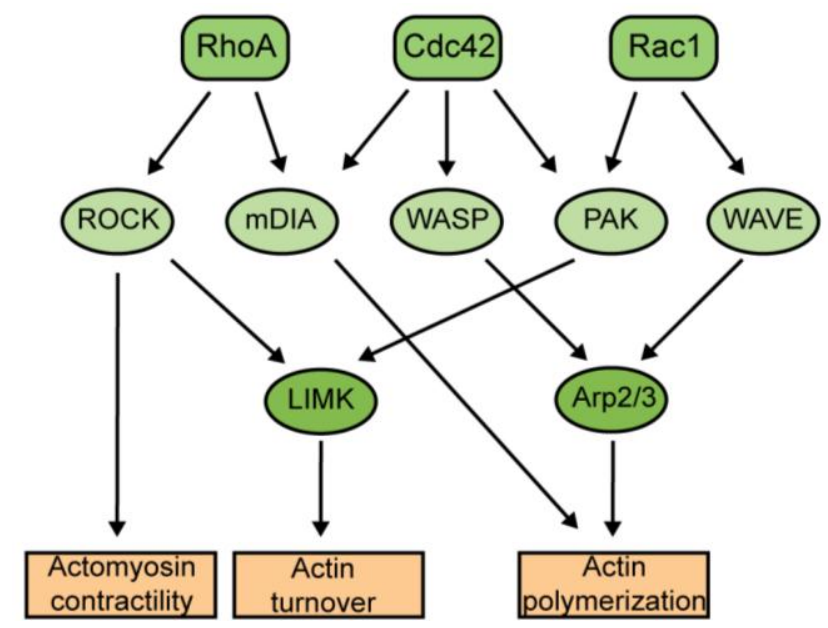

Abb 1.7: Rho-family of small GTPases regulating actin remodeling. RhoA promotes actomyosin contractility via ROCK. ROCK itself phosphorylates LIMK, thereby leading to inhibition of cofilin. RhoA also affects $\mathrm{mDIA}$, which in turn promotes actin polymerization. Rac1 antagonizes RhoA's function. It activates PAK and WAVE. WAVE-dependent activation of Arp2/3 leads to actin polymerization, whereas PAK-mediated activation of LIMK results in actin turnover. Cdc42 activates Arp2/3 via WASP resulting in actin polymerization. Adapted from Sadok \& Marshall 2014.

Altered expression or dysregulated activity of the Rho-family of small GTPases is frequently associated with tumorigenesis and the development of different cancer types including colorectal cancer (Mack et al. 2011). While no mutations for Rho GTPases have been described so far, the Rac1-specific GEF TIAM 1 (T-lymphoma invasion and metastasisinducing protein-1) and the RhoA-specific GEF RGNEF (p190RhoGEF) were shown to be up-regulated in colorectal cancer (Leve \& Morgado-Díaz 2012). TIAM 1 was identified as an invasion and metastasis gene and shown to be required for the initiation of colon cancer growth (Cook et al. 2013). In addition to invasion and metastasis, a mitotic role was also suggested for TIAM 1, since it was found to localize to mitotic centrosomes antagonizing the function of Eg5 in centrosome separation during prophase (Whalley et al. 2015). In axons, TIAM 1 was shown to localize to microtubules via MAP1B (Montenegro-Venegas et al. 2010). TRIO constitutes another Rac1-GEF with possible functions in mitosis. TRIO was identified as a microtubule plus-end binding protein in neurons (Van Haren et al. 2014). In these cells, binding is achieved via EB1/Nav1 complexes and requires dynamic microtubules. High expression of $T R I O$ is found in different tumor types, including breast and lung cancer and glioblastoma and is associated with poor patient prognosis (Schmidt \& Debant 2014).

A variety of tumor cells exhibit a deregulated expression or activity of Rho GTPases (Boettner \& Van Aelst 2002). Especially Rac1 hyperactivation is associated with aggressive tumor growth (Bid et al. 2013). Aggressive tumor growth is accompanied by a high migration and invasion potential. These processes require the formation of certain cell surface extensions like lamellipodia or invadopodia, which emerge from Rac1-mediated 
reorganization of the actin cytoskeleton (Parri \& Chiarugi 2010). Therefore, Rac1 or other members of the Rac1 pathway would represent interesting therapeutic targets for anti-cancer therapy (Bid et al. 2013).

\subsection{Cellular Migration}

The activation of invasion and metastasis represents a hallmark of cancer (Hanahan \& Weinberg 2011). Upon a migration promoting stimulus the cell starts to polarize and forms protrusions into the direction of migration. Thereby, the formation of protrusions is mainly driven by actin polymerization, whereas the establishment and maintenance of cell polarity is mediated by different factors like Rho-family GTPases, phosphoinositide 3-kinases (PI3Ks), vesicular transport and also microtubules (Ridley et al. 2003). Cell migration and invasion can be exemplified by a five step model (Lauffenburger \& Horwitz 1996; Friedl \& Wolf 2003): 1. Pseudopod protrusion at the leading edge, 2. Formation of a focal contact, 3. Focalized proteolysis, 4. Actomyosin contraction, 5. Detachment of the trailing edge (Fig. 1.8).

Cells can respond to very small differences of chemoattractant concentrations occurring between their front and rear. The signaling molecules Ptdlnst $(3,4,5) \mathrm{P}_{3} \quad\left(\mathrm{PIP}_{3}\right)$ and Ptdlnst $(3,4) \mathrm{P}_{2}\left(\mathrm{PIP}_{2}\right)$ rapidly polarize along this gradient, whereby $\mathrm{PIP}_{3}$ is located at the front and $\mathrm{PIP}_{2}$ at the rear and the sites of the cell. This leads to the accumulation and activation of PI3Ks and PTEN (Phosphatase and Tensin). PTEN is responsible for the cleavage of the 3' phosphate from $\mathrm{PIP}_{3}$ to generate $\mathrm{PIP}_{2}$. Thereby PTEN antagonizes the function of $\mathrm{PI} 3 \mathrm{~K}$ (Yamada \& Araki 2001). PI3K accumulates at the leading edge of a cell, whereas PTEN localization is confined to the cell's rear and sides. $\mathrm{PIP}_{3}$ and $\mathrm{PIP}_{2}$ have downstream effects on Rac1 and Cdc42 (Yamada \& Araki 2001). Several Rac1-GEFs are activated by PI3K products. Active Rac1 can then regulate itself via different positive feedback loops. On the one hand, active Rac1 is able to recruit and activate PI3Ks at the plasma membrane. On the other hand, microtubule polymerization activates Rac1 whereupon Rac1 stabilizes microtubules (Waterman-Storer et al. 1999). Both, Cdc42 and Rac1 stimulate actin polymerization by activating the Arp2/3 complex via the WASP or WAVE complex, leading to the nucleation of a highly branched actin network (Rohatgi et al. 1999; Lawson \& Burridge 2014), thereby inducing pseudopod extension (Nobes \& Hall 1995). The growing actin filaments bind to a complex consisting of a-actinin, vinculin and paxillin within the cell membrane (Calderwood et al. 2000). Continuous actin assembly pushes the cell membrane outwards. The growing cell protrusion touches the ECM and transmembrane receptors of the integrin family initiate binding to it. Actin filaments are coupled to integrins, which are accumulated and clustered at these sides and form a focal contact. These contacts are highly dynamic and can therefore stably adhere to or slowly glide along the substratum. 
Upon binding of integrins, surface proteases like MMP1 (matrix metalloproteinase 1) are recruited, which degrade ECM components like collagen, fibronectin and laminins (Ohuchi et al. 1997). During invasion, ECM degradation is an important process since it provides space for cell expansion and movement

1. Pseudopod protrusion at leading edge

2. Formation of focal contact

3. Focalized proteolysis

4. Actomyosin contraction
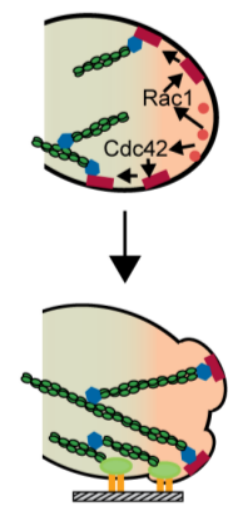

$\downarrow$
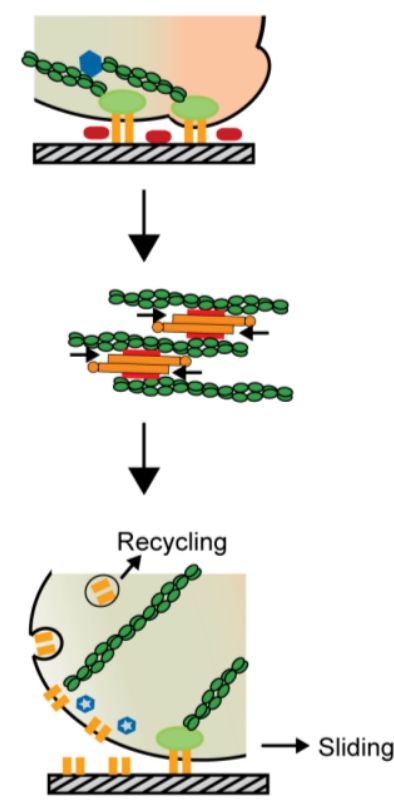

5. Detachment of trailing edge

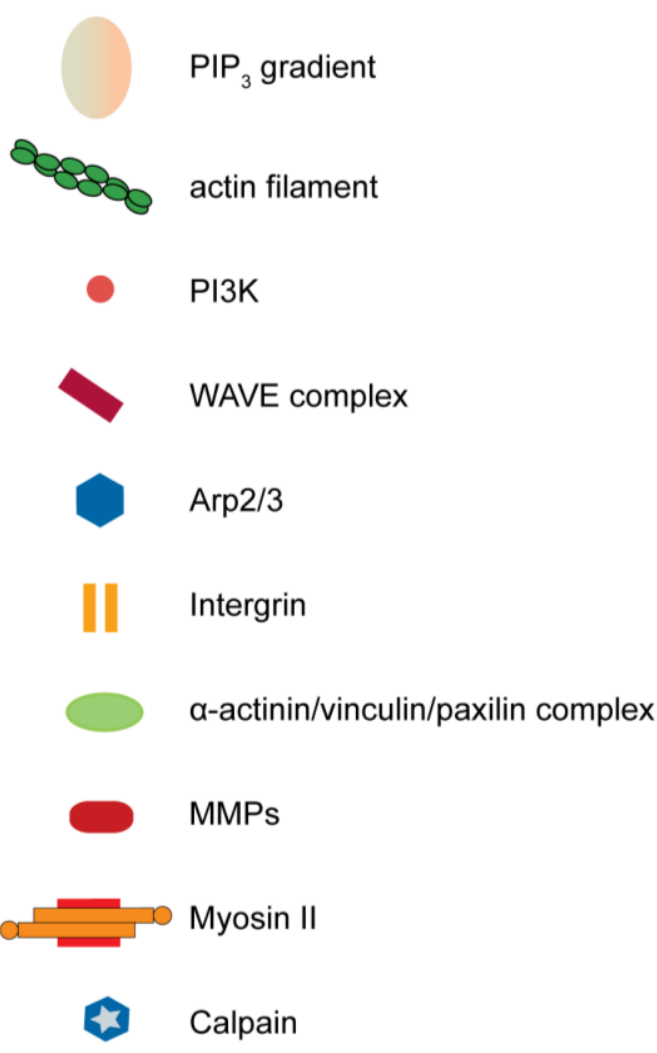

Figure 1.8: Model of cell migration. Migration can be divided into five steps: At first, the leading edge protrudes due to Arp2/3 mediated actin assembly. Second, a focal contact is formed via clustered integrins, whereupon surface proteases cleave ECM components during focalized proteolysis. The fourth step involves cell contraction by actomyosin, followed by the detachment of the trailing edge, which is mainly driven by focal contact disassembly. Modified from Friedl \& Wolf 2013

After the initial phase of adhesion, Rac1 activity in the lamellipodium diminishes whereas RhoA activity in the rear increases. RhoA promotes the formation of stress fibers and mediates the maturation of focal adhesions by activating myosin via Rho kinase (ROCK) (O'Connor \& Chen 2013; Lawson \& Burridge 2014). ROCK inactivates the myosin phosphatase by phosphorylating its myosin-binding subunit. Contraction of actin-bound 
myosin II, leads to bundling of actin fibers (O'Connor \& Chen 2013). Actin fibers are crosslinked by $\alpha$-actinin, thereby forming highly regulated actomyosin. Contraction of the actomyosin leads to shortening of the cell resulting in an inward tension towards the focal contacts (Chew et al. 2002). Cell-substrate-linkages are then resolved preferentially at the rear of the cell. Here, the cytoplasmic protease calpain cleaves focal contact components (Potter et al. 1997). In contrast, the leading edge stays attached and further elongates (Friedl \& Wolf 2003). After disassembly of the focal contact, the cell glides forward and detached integrins become recycled at the leading edge (Bretscher 1996).

\subsection{Microtubules In Cell Migration}

Microtubules are well described for their role in mitotic spindle assembly, but they are not only important during cell division, but also required to establish cell polarity during cell motility (Bershadsky et al. 1991). Already in 1970, the inhibitory effect of spindle poisons onto fibroblasts and their locomotory behavior was reported (Vasiliev et al. 1970). However not only a full depolymerization of microtubules but also abolished microtubule dynamics was detected to alter cell motility (Liao et al. 1995). Nowadays, it is well established, that microtubule dynamics polarize a motile cell in an asymmetric fashion. The main characteristic of the polarized microtubule cytoskeleton is its alignment with the cell migration axis (Etienne-Manneville 2013). Microtubules predominantly grow towards the leading edge and only few of them reach the cell's rear (Etienne-Manneville 2013). Furthermore, polarization is achieved by microtubule stabilization in the leading edge and microtubule destabilization at the trailing edge (Kaverina \& Straube 2011). At the front of a cell, microtubules are captured and stabilized at cortical sides by several +TIPs including CLASP1 and CLASP2 (Mimori-Kiyosue et al. 2005), CLIP-170 (Fukata et al. 2002) and EB3 (Straube \& Merdes 2007). Furthermore, leading edge microtubules are also target of Rho-GTPase regulation (Wittmann et al. 2003). On the one hand microtubules activate Rac1, thereby promoting lamellipodia formation, through delivering GEFs such as TIAM 1, TIAM 2 or TRIO (Waterman-Storer et al. 1999; Blangy et al. 2000; Rooney et al. 2010). But in a positive feedback loop, this enhanced activity of Rac1 further selectively promotes persistent growth of leading edge microtubules (Wittmann et al. 2003). Thereby, activation of Rac1 results in PAK1-mediated phosphorylation of the microtubule destabilizing protein Stathmin/Op18 (Daub et al. 2001). Due to this inactivation, catastrophe events are diminished and microtubule growth is promoted (Larsson et al. 1997).

At the rear of a cell, catastrophes are observed especially at focal adhesion sides (Bershadsky et al. 1996; Salaycik et al. 2005; Efimov et al. 2008). In fibroblasts, dynamic microtubules target mature focal adhesions. Due to their dynamic instability, microtubules 
can repeatedly contact a focal adhesion, resulting in its disassembly (Kaverina et al. 1998; Krylyshkina et al. 2003). Furthermore, depolymerizing microtubules at the trailing edge result in the activation of RhoA via its GEF GEF-H1 (Ren et al. 1999; Waterman-Storer et al. 1999), leading to the formation of stress fibers and increased contractility (Bershadsky et al. 1996). Thus, microtubules transduce signals to the cell migration machinery depending on their dynamic behavior (Kaverina \& Straube 2011): GEFs binding to microtubules can either be sequestered and inactivated by growing microtubules or released and activated by catastrophe events (Nalbant et al. 2010; Chang et al. 2007).

Hence, changes in microtubule dynamics implies alterations of cell migration (Kaverina \& Straube 2011). Microtubule associated proteins (MAPs) regulate the dynamic behavior of microtubules. EB1 and APC stabilize microtubules at the leading edge in a RhoA-mDiamediated fashion (Wen et al. 2004). EB1 and EB3 were described to suppress catastrophes but they promote persistent microtubule growth (Komarova et al. 2009). EB1 was also shown to increase membrane protrusion and cell migration in melanoma cells (Schober et al. 2009). In these cells, depletion of EB1 resulted in a loss of polarized cell morphology. Furthermore, APC accumulates at leading edge microtubules, promotes their growth and diminishes shrinkage, thus exerting a stabilizing function (Kita et al. 2006). Interestingly, microtubules in the leading edge are often post-translationally modified by acetylations or detyrosination (Gundersen \& Bulinski 1988), resulting in increased stability (Tran et al. 2007) and a potentially increased recruitment of MAPs (Etienne-Manneville 2013).

Additionally, microtubules serve as tracks for directed membrane and organelle transport, thereby providing building material for protrusion formation (Nabi 1999) as well as molecular motors for focal adhesion turnover (Krylyshkina et al. 2002), which is mediated by integrin recycling (Pellinen \& Ivaska 2006). Furthermore, endosomes carry membrane-associated molecules such as Rac1 and Cdc42, that are delivered to the plasma membrane (Palamidessi et al. 2008; Osmani et al. 2010). The traffic is facilitated by the existence of long microtubule tracks between the cell center and the periphery (Komarova et al. 2009).

In cancer, the microtubule-dependent control of cell migration is often impaired (Kaverina \& Straube 2011).

\subsection{Cell Invasion}

The motility of a cell is often studied in $2 \mathrm{D}$, where a cell migrates on a substratum towards a chemoattractant forming filopodia and lamellipodia. In contrast, invasion is the process of moving into or within a 3D matrix (Fife et al. 2014), which can arise from an aberrant regulation of cell migration (Sahai 2005; Yamaguchi et al. 2005). The invasion process occurs during cell morphogenesis, wound healing and in malignant cells allowing the 
metastatic growth into distant organs (Friedl \& Wolf 2003). Therefore, tumor cells need to detach from the primary tumor, invade the surrounding tissue, intravasate the blood or lymphatic vessels, extravasate into distant organs and grow to form secondary tumors (Hofmann et al. 2000). For these processes, specialized structures like invadopodia and podosomes and the proteolysis of the extracellular matrix (ECM) are required (Friedl \& Wolf 2003). Podosomes are sides of contact to the substratum similar to focal adhesions, but they differ in some criteria. In contrast to focal adhesions, podosomes contain actin organizing structures like the Arp2/3 complex, N-WASP and fimbrin, which is an actin bundling protein (Buccione et al. 2004). Furthermore, they are more dynamic and form from pre-existing podosomes and therefore do not require ongoing protein synthesis (Buccione et al. 2004). Podosomes continuously probe the substratum and invadopodia are formed by their extension (Buccione et al. 2004). Invadopodia are membrane protrusions with ECM degradation activity (Buccione et al. 2004) and their formation is dependent on Arp2/3 activity. The knock down of Arp2/3 was shown suppress the development of these membrane protrusions (Yamaguchi et al. 2005). In malignant cells, an excessive cell protrusive activity was found, which is mainly driven by aberrant signaling regarding the activation of actin rearrangements (Wang et al. 2004). This signature of invasion involved deregulated genes in mammary tumors further includes LIMK1 (LIM-kinase 1), CFL1 (cofilin) and ACTN3 (actinin) (Wang et al. 2004).

The degradation and remodeling of the ECM requires matrix-metalloproteinases (MMPs) (Coussens \& Werb 1996; Chambers \& Matrisian 1997). Several MMPs interact with surface receptors like integrins or are located to the ECM (Kessenbrock et al. 2010). The upregulation of MMPs is often found in human cancers including breast cancer and melanoma (Rudolph-Owen et al. 1998; Hofmann et al. 2000). Interestingly, in breast cancer, the activation of MMPs was implicated in tumor progression and promotion of EMT (Radisky \& Radisky 2010). The direct activation of the EMT program is mediated by MMP-3 stimulated expression of a constitutively active Rac1 splice variant (Rac1b), which increases reactive oxygen species in the cell, resulting in expression of SNAIL and induction of EMT (Radisky et al. 2005). Furthermore, MMP-3 is able to cleave E-cadherin, resulting in a bioactive Ecadherin fragment, which is capable of stimulating cancer cell motility (Lochter et al. 1997; Noë et al. 2001).

\subsection{Epithelial-Mesenchymal-Transition (EMT)}

Cancer cells invade into tissues by moving as single epithelial or amoeboid cells or as clusters. Thereby, the process of invasion accompanies morphological and phenotypical changes (Van Zijl et al. 2011). 
The epithelial-mesenchymal-transition (EMT) is a conserved mechanism occurring during embryogenesis, chronic inflammation and fibrosis, but it is also a common process during cancer progression and the development of metastasis (Van Zijl et al. 2011). A hallmark of EMT is the loss of epithelial characteristics. Instead, the cell gains mesenchymal features (Spaderna et al. 2006). As a result, cells destabilize cell-cell-contacts due to the downregulation of $C D H 1$, encoding for epithelial E-cadherin (Thiery \& Sleeman 2006). Ecadherin exhibits an extracellular domain, which enables binding to E-cadherins of a neighboring cell. Downregulation of $\mathrm{CDH} 1$ is mediated by the transcriptional repressors Snail/SNAI1, Slug/SNAI2, SIP1/ZEB2 or Twist. The disassembly of adherence junctions leads to the translocation of membrane bound $\beta$-catenin into the nucleus. There, it affects the transcription of genes like c-myc or cyclin D (Van Zijl et al. 2011). Subsequently, mesenchymal markers are upregulated. Among them, $\mathrm{N}$-cadherin is responsible for the formation of Rho-induced stress fibers and activation of Rac1 and Cdc42, leading to the development of filopodia and lamellipodia. Cells, that have undergone EMT, are also able to regain epithelial properties. The mesenchymal-epithelial-transition (MET) leads to epithelial reorganization resulting in the ability for colonization at secondary sites, which is an important process during metastasis (Van Zijl et al. 2011). Thus, EMT can contribute or even be a prerequisite for cancer cell migration and invasion.

\section{Scope Of The Study}

Chromosomal instability is a hallmark of most solid tumors (Orr \& Compton 2013) and has been associated with tumorigenesis and the development of therapy resistance (Bakhoum \& Compton 2012). CIN enables the development of genetic heterogeneity and thus the emergence of novel phenotypes in cancer cells. Among them, cell migration and invasion are major characteristics that are acquired during the development of aggressive metastatic human tumors (Hanahan \& Weinberg 2000). Both, CIN and cancer cell invasion are associated with tumor stage and poor patient prognosis. Although both phenotypes often appear concomitantly in late tumor stages, little is known about a possible shared link between CIN and cancer cell migration and invasion. Thus, the aim of this study was to investigate a potential shared mechanistic link between the development of CIN and a highly migratory and invasive phenotype. 


\section{Material and Methods}

\subsection{Material}

The used working materials including cell culture dishes, pipette tips, disposable syringes and reaction tubes were purchased from Sarstedt (Nümbrecht, Germany), Greiner BioOne (Frickenhausen, Germany), Eppendorf (Hamburg, Germany) and Ibidi (Martinsried, Germany).

\subsubsection{Equipment}

Table 2.1 lists the equipment, which was used for the implementation of this study.

Table 2.1: Equipment

\begin{tabular}{|c|c|c|}
\hline Equipment & Model & Company \\
\hline $\mathrm{CO}_{2}$ Incubator & HERAcell $240 \mathrm{CO}_{2}$ Incubator & $\begin{array}{l}\text { Thermo Fisher Scientific, Karlsruhe, } \\
\text { Germany }\end{array}$ \\
\hline Cooling Centrifuge & Multifuge X3R & $\begin{array}{l}\text { Thermo Fisher Scientific, Karlsruhe, } \\
\text { Germany }\end{array}$ \\
\hline $\begin{array}{l}\text { Electroporation } \\
\text { Device }\end{array}$ & GenePulser Xcell ${ }^{\odot}$ & $\begin{array}{l}\text { BioRad Laboratories, München, } \\
\text { Germany }\end{array}$ \\
\hline $\begin{array}{l}\text { Electrophoresis } \\
\text { Power Supply }\end{array}$ & Power Supply EV231 & Peqlab, Erlangen, Germany \\
\hline Flow Cytometer & BD FACSCanto $^{\odot} \|$ & $\begin{array}{l}\text { Becton Dickinson, San Jose, CA, } \\
\text { USA }\end{array}$ \\
\hline Gel Documentation & Gel iX Imager & Intas, Göttingen, Germany \\
\hline \multirow{2}{*}{ Heating Block } & Thermomixer Comfort $\mathrm{R}$ & Eppendorf, Hamburg, Germany \\
\hline & $\begin{array}{l}\text { TDB-120 Dry Block } \\
\text { Thermostat }\end{array}$ & Biosan, Riga, Latvia \\
\hline $\begin{array}{l}\text { Horizontal Gel } \\
\text { Electrophoresis } \\
\text { System }\end{array}$ & Sub-Cell GT cell & $\begin{array}{l}\text { BioRad Laboratories, München, } \\
\text { Germany }\end{array}$ \\
\hline Laboratory Scale & Sartorius Research R200D & Sartorius, Göttingen, Germany \\
\hline Magnetic Mixer & $\mathrm{IKAMAG}^{\odot} \mathrm{RCT}$ & IKA Labortechnik, Stauffen, Germany \\
\hline Medical X-Ray Film & Fuji Super RX & $\begin{array}{l}\text { Christiansen und Linhardt, Planegg, } \\
\text { Germany }\end{array}$ \\
\hline
\end{tabular}




\begin{tabular}{|c|c|c|}
\hline Equipment & Model & Company \\
\hline \multirow{3}{*}{ Microscope } & Delta Vision Elite ${ }^{\odot}$ & $\begin{array}{l}\text { Applied Precision, Chalfont St. Giles, } \\
\text { UK }\end{array}$ \\
\hline & Leica DMI6000B & Leica, Wetzlar, Germany \\
\hline & Zeiss Axio Imager Z1 & Zeiss, Göttingen, Germany \\
\hline \multirow{3}{*}{ Microscope Camera } & sCMOS camera & $\begin{array}{l}\text { GE Healthcare, Chalfront St. Giles, } \\
\text { UK }\end{array}$ \\
\hline & Leica DFC369 FX & Leica, Wetzlar, Germany \\
\hline & $\begin{array}{l}\text { Hamamatsu } 1394 \text { ORCA-II } \\
\text { ER }\end{array}$ & $\begin{array}{l}\text { Hamamatsu Photonics, Hamamatsu, } \\
\text { Japan }\end{array}$ \\
\hline $\begin{array}{l}\text { Microscope Camera } \\
\text { Adaptor }\end{array}$ & A3474-07 & $\begin{array}{l}\text { Hamamatsu Photonics, Hamamatsu, } \\
\text { Japan }\end{array}$ \\
\hline Multilabel Reader & Victor $^{\odot}$ X3 & PerkinElmer, Rodgau, Germany \\
\hline $\begin{array}{l}\text { Nitrocellulose } \\
\text { Membrane }\end{array}$ & Protran BA 83 & $\begin{array}{l}\text { GE Healthcare, Chalfront St. Giles, } \\
\text { Great Britain }\end{array}$ \\
\hline Pipettes & Pipetman $^{\odot}$ & $\begin{array}{l}\text { Gilson International, LimburgOffheim, } \\
\text { Germany }\end{array}$ \\
\hline Pipettor & Pipetboy acu & $\begin{array}{l}\text { Integra Biosciences, Fernwald, } \\
\text { Germany }\end{array}$ \\
\hline PVDF Membrane & ImmobilionR-P & Merck Millipore, Darmstadt, Germany \\
\hline $\begin{array}{l}\text { Semidry Western } \\
\text { Blotting Device }\end{array}$ & Perfect Blue ${ }^{\odot}$ & Peqlab, Erlangen, Germany \\
\hline Sterile Workbench & HERAsafeM & $\begin{array}{l}\text { Thermo Fisher Scientific, Karlsruhe, } \\
\text { Germany }\end{array}$ \\
\hline Spectrophotometer & NanoDrop 2000 & $\begin{array}{l}\text { Thermo Fisher Scientific, Karlsruhe, } \\
\text { Germany }\end{array}$ \\
\hline Tabletop Centrifuge & Biofuge pico & $\begin{array}{l}\text { Thermo Fisher Scientific, Karlsruhe, } \\
\text { Germany }\end{array}$ \\
\hline $\begin{array}{l}\text { Tabletop Centrifuge, } \\
\text { cooling }\end{array}$ & Biofuge fresco & $\begin{array}{l}\text { Thermo Fisher Scientific, Karlsruhe, } \\
\text { Germany }\end{array}$ \\
\hline $\begin{array}{l}\text { Vertical } \\
\text { Electrophoresis } \\
\text { System }\end{array}$ & & Own Manufacturing \\
\hline Vortex Mixer & VORTEX-GENIE ${ }^{\odot} 2$ & $\begin{array}{l}\text { Scientific Industries inc., Bohemia, } \\
\text { NY, USA }\end{array}$ \\
\hline X-Ray film processor & Optimax & Protec, Oberstenfeld, Germany \\
\hline
\end{tabular}




\subsubsection{Software}

The following table lists the software, which was used for data analyses.

Table 2.2: Software

\begin{tabular}{ll} 
Software & Company \\
\hline \hline Hokawo Launcher & Hamamatsu Photonics, Hamamatsu, Japan \\
\hline ImageJ & NIH Image, Bethesda, MD, USA \\
\hline Leica Application Suite & Leica, Wetzlar, Germany \\
\hline Soft Worx ${ }^{\odot} 5.0$ & Applied Precision Inc., Issaquah, WA, USA \\
\hline
\end{tabular}

\subsubsection{Chemicals}

All used chemicals were purchased from Carl Roth (Karlsruhe, Germany), Sigma-Aldrich (Taufkirchen, Germany), VWR International (West Chester, PA, USA), BD Biosciences (Heidelberg, Germany), Fermentas (St. Leon-Roth, Germany), Promega (Madison, WI, USA), Merck Millipore (Darmstadt, Germany), Roche Diagnostics (Mannheim, Germany), Thermo Fisher Scientific (Waltham, MA, USA), Th. Geyer (Renningen, Germany), Enzo Life Sciences (New York, NY, USA) AppliChem (Darmstadt, Germany), Amersham Biosciences (Buckinghamshire, Great Britain).

Table 2.3 lists chemicals and inhibitors used in this study and their respective working concentration.

Table 2.3: Chemicals

\begin{tabular}{llll} 
Chemical & $\begin{array}{l}\text { Used } \\
\text { concentration }\end{array}$ & Effect & Company \\
\hline \hline Adriamycin & $600 \mathrm{nM}$ & $\begin{array}{l}\text { Intercalates into DNA } \\
\text { and stabilizes } \\
\text { topoisomerase II }\end{array}$ & Th. Geyer, Höxter, Germany \\
\hline CK666 & $20 \mu \mathrm{M}$ & $\begin{array}{l}\text { Inhibition of actin } \\
\text { assembly by the Arp2/3 } \\
\text { complex }\end{array}$ & $\begin{array}{l}\text { Merck Millipore, Darmstadt, } \\
\text { Germany }\end{array}$ \\
\hline Dimethylenastron & $2 \mu \mathrm{M}$ & Inhibition of EG5-kinesin & $\begin{array}{l}\text { Calbiochem, La Jolla, CA, } \\
\text { USA }\end{array}$ \\
\hline Doxycycline & $200 \mathrm{ng} / \mathrm{ml}$ & $\begin{array}{l}\text { Induction of gene } \\
\text { expression }\end{array}$ & $\begin{array}{l}\text { Sigma-Aldrich Taufkirchen, } \\
\text { Germany }\end{array}$ \\
\hline ITX3 & $15 \mu \mathrm{M}$ & $\begin{array}{l}\text { Inhibition of GEF-activity } \\
\text { of TRIO }\end{array}$ & $\begin{array}{l}\text { Merck Millipore, Darmstadt, } \\
\text { Germany }\end{array}$ \\
\hline
\end{tabular}




\begin{tabular}{llll} 
Chemical & $\begin{array}{l}\text { Used } \\
\text { concentration }\end{array}$ & Effect & Company \\
\hline \hline Nocodazol & $2-300 \mathrm{nM}$ & $\begin{array}{l}\text { Depolymerization of } \\
\text { microtubules }\end{array}$ & $\begin{array}{l}\text { Sigma-Aldrich Taufkirchen, } \\
\text { Germany }\end{array}$ \\
\hline NSC23766 & $40 \mu \mathrm{M}$ & Inhibition of Rac1 & $\begin{array}{l}\text { Santa Cruz, Dallas, TX, } \\
\text { USA }\end{array}$ \\
\hline Ponasterone A & $5 \mu \mathrm{mol} / \mathrm{l}$ & $\begin{array}{l}\text { Induction of gene } \\
\text { expression }\end{array}$ & $\begin{array}{l}\text { Sigma-Aldrich Taufkirchen, } \\
\text { Germany }\end{array}$ \\
\hline Puromycin & $1 \mu \mathrm{g} / \mathrm{ml}$ & Inhibition of translation & $\begin{array}{l}\text { Sigma-Aldrich Taufkirchen, } \\
\text { Germany }\end{array}$ \\
\hline Taxol & $0.2-150 \mathrm{nM}$ & $\begin{array}{l}\text { Stabilization of } \\
\text { microtubules }\end{array}$ & $\begin{array}{l}\text { Sigma-Aldrich Taufkirchen, } \\
\text { Germany }\end{array}$ \\
\hline $\begin{array}{l}\text { TGF- } \beta \text { (human, } \\
\text { recombinant) }\end{array}$ & $10 \mathrm{ng} / \mathrm{ml}$ & $\begin{array}{l}\text { Stimulation of TGF- } \beta \\
\text { signaling }\end{array}$ & $\begin{array}{l}\text { PeproTech, Hamburg, } \\
\text { Germany }\end{array}$ \\
\hline Thymidine & $2 \mathrm{mM}$ & $\begin{array}{l}\text { Inhibition of nucleotide } \\
\text { synthesis }\end{array}$ & $\begin{array}{l}\text { Sigma-Aldrich Taufkirchen, } \\
\text { Germany }\end{array}$ \\
\hline
\end{tabular}

\subsubsection{Antibodies}

\section{Primary Antibodies}

Primary antibodies, including their host species, antibody type and the used dilutions are listed in Table 2.4.

Table 2.4: Primary Antibodies

\begin{tabular}{|c|c|c|c|c|}
\hline Antigen & $\begin{array}{l}\text { Host } \\
\text { Species }\end{array}$ & $\begin{array}{l}\text { Antibody } \\
\text { Type }\end{array}$ & Used Dilution & Company \\
\hline $\begin{array}{l}\alpha-T u b u l i n(B-5-1- \\
\text { 2) }\end{array}$ & mouse & monoclonal & IF 1:700 & Santa Cruz, Dallas, TX, USA \\
\hline$\beta$-Actin (A5441) & mouse & monoclonal & WB 1:5000 & $\begin{array}{l}\text { Sigma-Aldrich, Taufkirchen, } \\
\text { Germany }\end{array}$ \\
\hline $\begin{array}{l}\text { ch-TOG } \\
(\mathrm{H}-4)\end{array}$ & mouse & monoclonal & WB 1:750 & Santa Cruz, Dallas, TX, USA \\
\hline $\begin{array}{l}\text { EB1 } \\
\text { (clone 5) }\end{array}$ & mouse & monoclonal & WB 1:500 & $\begin{array}{l}\text { BD Biosciences, Heidelberg, } \\
\text { Germany }\end{array}$ \\
\hline $\begin{array}{l}\text { E-Cadherin } \\
\text { (clone 36) }\end{array}$ & Mouse & Monoclonal & WB 1:1000 & $\begin{array}{l}\text { BD Biosciences, Heidelberg, } \\
\text { Germany }\end{array}$ \\
\hline $\begin{array}{l}\text { y-Tubulin } \\
\text { (T3559) }\end{array}$ & rabbit & polyclonal & IF $1: 700$ & $\begin{array}{l}\text { Sigma-Aldrich, Taufkirchen, } \\
\text { Germany }\end{array}$ \\
\hline $\begin{array}{l}\text { y-Tubulin } \\
\text { (T6557) }\end{array}$ & mouse & monoclonal & WB 1:2000 & $\begin{array}{l}\text { Sigma-Aldrich, Taufkirchen, } \\
\text { Germany }\end{array}$ \\
\hline $\begin{array}{l}\text { Human Nuclear } \\
\text { ANA- } \\
\text { Centromere } \\
\text { (CREST) }\end{array}$ & human & polyclonal & IF $1: 700$ & $\begin{array}{l}\text { Europa Bioproducts, Wicken, } \\
\text { Ely, UK }\end{array}$ \\
\hline
\end{tabular}




\begin{tabular}{|c|c|c|c|c|}
\hline Antigen & $\begin{array}{l}\text { Host } \\
\text { Species }\end{array}$ & $\begin{array}{l}\text { Antibody } \\
\text { Type }\end{array}$ & Used Dilution & Company \\
\hline MCAK & sheep & polyclonal & WB 1:250 & $\begin{array}{l}\text { Kindly provided by Prof. Linda } \\
\text { Wordemann (Seattle, WA, } \\
\text { USA) }\end{array}$ \\
\hline Mep21 & mouse & polyclonal & IHC 1:500 & $\begin{array}{l}\text { Kindly provided by Prof. Jörg } \\
\text { Wilting (Göttingen, Germany) }\end{array}$ \\
\hline $\begin{array}{l}\mathrm{p} 21 \\
(\mathrm{Ab}-1)\end{array}$ & mouse & monoclonal & WB 1:1000 & $\begin{array}{l}\text { Oncogene, Cambridge, MA, } \\
\text { USA }\end{array}$ \\
\hline $\begin{array}{l}\mathrm{p} 53 \\
(\mathrm{Ab}-6)\end{array}$ & mouse & monoclonal & WB 1:800 & $\begin{array}{l}\text { Oncogene, Cambridge, MA, } \\
\text { USA }\end{array}$ \\
\hline $\begin{array}{l}\text { p73 } \\
\text { (EP436y) }\end{array}$ & rabbit & monoclonal & WB 1:1000 & GeneTex, Irvine, CA, USA \\
\hline $\begin{array}{l}\text { Rac1 } \\
\text { (Arc03) }\end{array}$ & mouse & monoclonal & WB $1: 500$ & $\begin{array}{l}\text { Cytoskeleton Inc., Denver, } \\
\text { CO, USA }\end{array}$ \\
\hline $\begin{array}{l}\text { TRIO } \\
\text { (D-20) }\end{array}$ & goat & Polyclonal & WB $1: 300$ & Santa Cruz, Dallas, TX, USA \\
\hline
\end{tabular}

\section{Secondary Antibodies}

Table 2.5 lists the used secondary antibodies including the host species, antibody type, the conjugated molecule and the used concentration.

Table 2.5: Secondary Antibodies

\begin{tabular}{|c|c|c|c|c|c|}
\hline Antigen & Species & $\begin{array}{l}\text { Antibody } \\
\text { Type }\end{array}$ & Conjugate & $\begin{array}{l}\text { Used } \\
\text { Dilution }\end{array}$ & Company \\
\hline Anti-Human & goat & polyclonal & $\begin{array}{l}\text { Alexa- } \\
\text { Fluor594 }\end{array}$ & IF $1: 1000$ & $\begin{array}{l}\text { Invitrogen, Carlsbad, } \\
\text { CA, USA }\end{array}$ \\
\hline Anti-Mouse & goat & polyclonal & $\begin{array}{l}\text { Alexa- } \\
\text { Fluor488 }\end{array}$ & IF 1:1000 & $\begin{array}{l}\text { Invitrogen, Carlsbad, } \\
\text { CA, USA }\end{array}$ \\
\hline Anti-Rabbit & goat & polyclonal & $\begin{array}{l}\text { Alexa- } \\
\text { Fluor594 }\end{array}$ & IF $1: 1000$ & $\begin{array}{l}\text { Invitrogen, Carlsbad, } \\
\text { CA, USA }\end{array}$ \\
\hline Anti-Goat & mouse & polyclonal & $\begin{array}{l}\text { Horseradish } \\
\text { Peroxidase } \\
(\mathrm{HRP})\end{array}$ & WB 1:10000 & $\begin{array}{l}\text { Dianova, Hamburg, } \\
\text { Germany }\end{array}$ \\
\hline Anti-Mouse & goat & polyclonal & $\begin{array}{l}\text { Horseradish } \\
\text { Peroxidase } \\
(\mathrm{HRP})\end{array}$ & WB 1:10000 & $\begin{array}{l}\text { Dianova, Hamburg, } \\
\text { Germany }\end{array}$ \\
\hline Anti-Rabbit & goat & polyclonal & $\begin{array}{l}\text { Horseradish } \\
\text { Peroxidase } \\
(\mathrm{HRP})\end{array}$ & WB 1:10000 & $\begin{array}{l}\text { Dianova, Hamburg, } \\
\text { Germany }\end{array}$ \\
\hline Anti-Sheep & goat & polyclonal & $\begin{array}{l}\text { Horseradish } \\
\text { Peroxidase } \\
\text { (HRP) }\end{array}$ & WB 1:10000 & $\begin{array}{l}\text { Dianova, Hamburg, } \\
\text { Germany }\end{array}$ \\
\hline
\end{tabular}




\subsubsection{Chromosome Enumeration Probes}

The used chromosome enumeration probes including the chromosome region of their hybridisation are listed in Table 2.6.

Table 2.6: Chromosome Enumeration Probes

\begin{tabular}{lcl} 
Chromosome & Chromosome Region & Company \\
\hline \hline 6 & $6 \mathrm{p} 11.1-\mathrm{q} 11.1$ & Cytocell aquarius, Cambridge, UK \\
\hline 7 & $7 \mathrm{p} 11.1-\mathrm{q} 11.1$ & Cytocell aquarius, Cambridge, UK \\
\hline 15 & $15 \mathrm{p} 11.1-q 11.1$ & Cytocell aquarius, Cambridge, UK \\
\hline 18 & $18 \mathrm{p} 11.1-q 11.1$ & Cytocell aquarius, Cambridge, UK
\end{tabular}

\subsubsection{Oligonucleotides}

The oligonucleotids used in this study were synthesized by Sigma-Aldrich (Taufkirchen, Germany) and stored at $-20^{\circ} \mathrm{C}$.

\section{qRT-PCR Primer}

The qRT-PCR primer used for this study and their respective sequence are listed in Table 2.7.

Table 2.7: qRT-PCR Primer

\begin{tabular}{|c|c|c|}
\hline Gene & Primer & Reference \\
\hline \multirow{2}{*}{$36 B 4$} & forward 5'-GATTGGCTACCCAACTGTTG-3' & Kindly provided by Matthias \\
\hline & reverse 5'-CAGGGGCAGCAGCCACAAA-3' & Dobbelstein (Göttingen, Germany) \\
\hline$C D K N 1 A$ & $\begin{array}{ll}\text { forward } & 5 ' \text {-TAGGCGGTTGAATGAGAGG-3 } \\
\text { reverse } & 5 \\
\end{array}$ & $\begin{array}{l}\text { Kindly provided by Matthias } \\
\text { Dobbelstein (Göttingen, Germany) }\end{array}$ \\
\hline
\end{tabular}


SIRNA

Table 2.8 lists the used siRNAs, their sequence and the corresponding reference.

Table 2.8: siRNAs

\begin{tabular}{|c|c|c|}
\hline Target Gene & Sequence & Reference \\
\hline CDKN1A & 5'-AAUCCCAGCUACUUGGAAGGC-3' & Zhang et al. 2005 \\
\hline CKAP5 & 5'-GAGCCCAGAGTGGTCCAAA-3' & De Luca et al. 2008 \\
\hline$E B 1$ & 5'-AUUCCAAGCUAAGCUAGAA-3' & Watson \& Stephens 2006 \\
\hline LUCIFERASE & 5'-CUUACGCUGAGUACUUCGAUU-3' & Elbashir et al. 2001 \\
\hline$R B$ & 5'-AAGUUUCAUCUGUGGAUGGAG-3' & Guo et al. 2011 \\
\hline \multirow{2}{*}{ TP53 } & 5'-GUAAUCUACUGGGACGGAA-3' & Ertych et al. 2014 \\
\hline & 5'-GACUCCAGUGGUAAUCUAC-3' & Brummelkamp et al. 2002 \\
\hline \multirow{4}{*}{ TP73 } & 5'-CAGGUGACCGACGUCGUGAAA-3' & \multirow{4}{*}{ Qiagen, Hilden, Germany } \\
\hline & 5'-CUCGGGAGGGACUUCAACGAA-3' & \\
\hline & 5'-CCCGGGAUGCUCAACAACCAU-3' & \\
\hline & 5'-CCCGCUCUUGAAGAAACUCUA-3' & \\
\hline \multirow[b]{2}{*}{ TRIO } & 5'-GAUAAGAGGUACAGAGAUU-3' & \multirow{2}{*}{ Cannet et al. 2014} \\
\hline & 5'-GGAAGUCGCUCCUUGACAA-3' & \\
\hline
\end{tabular}

\subsubsection{Plasmids}

The used plasmids including their purpose and reference are listed in Table 2.9.

Table 2.9: Plasmids

\begin{tabular}{lll} 
Vector & Purpose & Reference \\
\hline \hline pcDNA3.1 & $\begin{array}{l}\text { CMV-promotor driven } \\
\text { expression vector for human } \\
\text { cells }\end{array}$ & Invitrogen, Carlsbad, CA, USA \\
\hline pcDNA3.1- $\triangle$ Np73 & $\begin{array}{l}\text { CMV-promotor driven } \\
\text { expression of } \Delta \text { Np73 in } \\
\text { human cells }\end{array}$ & $\begin{array}{l}\text { Kindly provided by Prof. } \\
\text { Matthias Dobbelstein } \\
\text { (Göttingen, Germany) }\end{array}$ \\
\hline
\end{tabular}




\begin{tabular}{|c|c|c|}
\hline Vector & Purpose & Reference \\
\hline pcDNA3.1-E1A & $\begin{array}{l}\text { CMV-promotor driven } \\
\text { expression of } E 1 A \text { in human } \\
\text { cells }\end{array}$ & $\begin{array}{l}\text { Kindly provided by Prof. } \\
\text { Matthias Dobbelstein } \\
\text { (Göttingen, Germany) }\end{array}$ \\
\hline pBabe-puro-HPV16-E7 & $\begin{array}{l}\text { CMV-promotor driven } \\
\text { expression of } E 7 \text { in human } \\
\text { cells }\end{array}$ & $\begin{array}{l}\text { Kindly provided by Prof. } \\
\text { Matthias Dobbelstein } \\
\text { (Göttingen, Germany) }\end{array}$ \\
\hline pcDNA3.1-MCAK & $\begin{array}{l}\text { CMV-promotor driven } \\
\text { expression of MCAK in } \\
\text { human cells }\end{array}$ & $\begin{array}{l}\text { Kindly provided by Prof. Linda } \\
\text { Wordeman (Seattle, WA, USA) }\end{array}$ \\
\hline pcDNA3.1-CDKN1A & $\begin{array}{l}\text { CMV-promotor driven } \\
\text { expression of TP21 in } \\
\text { human cells }\end{array}$ & $\begin{array}{l}\text { Kindly provided by Prof. } \\
\text { Matthias Dobbelstein } \\
\text { (Göttingen, Germany) }\end{array}$ \\
\hline pEGFP-C1 & $\begin{array}{l}\text { CMV-promotor driven } \\
\text { expression vector for human } \\
\text { cells }\end{array}$ & $\begin{array}{l}\text { Clontech, Saint-Germain-en- } \\
\text { Laye, France }\end{array}$ \\
\hline pEGFP-CKAP5 & $\begin{array}{l}\text { CMV-promotor driven } \\
\text { expression of GFP-tagged } \\
\text { CKAP5 in human cells }\end{array}$ & $\begin{array}{l}\text { Kindly provided by Prof. Linda } \\
\text { Wordeman (Seattle, WA, USA) }\end{array}$ \\
\hline pEGFP-EB3 & $\begin{array}{l}\text { CMV-promotor driven } \\
\text { expression of GFP-tagged } \\
E B 3 \text { in human cells }\end{array}$ & $\begin{array}{l}\text { Kindly provided by Prof. Linda } \\
\text { Wordeman (Seattle, WA, USA) }\end{array}$ \\
\hline pEGFP-HMMR & $\begin{array}{l}\text { CMV-promotor driven } \\
\text { expression of GFP-tagged } \\
\text { HMMR in human cells }\end{array}$ & $\begin{array}{l}\text { Kindly provided by Prof. Tony } \\
\text { Reiman (Westmount, Canada) }\end{array}$ \\
\hline pEGFP-mCherry-EB3 & $\begin{array}{l}\text { CMV-promotor driven } \\
\text { expression of mCherry- } \\
\text { tagged } E B 3 \text { in human cells }\end{array}$ & $\begin{array}{l}\text { Kindly provided by Prof. Linda } \\
\text { Wordeman (Seattle, WA, USA) }\end{array}$ \\
\hline pEGFP-Rac1 Q61L & $\begin{array}{l}\text { CMV-promotor driven } \\
\text { expression of GFP-tagged } \\
\text { constitutively active Rac1 in } \\
\text { human cells }\end{array}$ & $\begin{array}{l}\text { Kindly provided by Prof. Robert } \\
\text { Grosse (Marburg, Germany) }\end{array}$ \\
\hline pEGFP-Rac1 T17N & $\begin{array}{l}\text { CMV-promotor driven } \\
\text { expression of GFP-tagged } \\
\text { dominant negative Rac1 in } \\
\text { human cells }\end{array}$ & $\begin{array}{l}\text { Kindly provided by Prof. Robert } \\
\text { Grosse (Marburg, Germany) }\end{array}$ \\
\hline plnducer & $\begin{array}{l}\text { Doxycycline inducible } \\
\text { expression vector for human } \\
\text { cells }\end{array}$ & $\begin{array}{l}\text { Kindly provided by Prof. } \\
\text { Thorsten Stiewe (Marburg, } \\
\text { Germany) }\end{array}$ \\
\hline plnducer20-TAp73a & $\begin{array}{l}\text { Doxycycline inducible } \\
\text { expression of } T A p 73 \alpha \text { in } \\
\text { human cells }\end{array}$ & $\begin{array}{l}\text { Kindly provided by Prof. } \\
\text { Thorsten Stiewe (Marburg, } \\
\text { Germany) }\end{array}$ \\
\hline pLOK.1-sh-TP73 & $\begin{array}{l}\text { Expression of a TP73 } \\
\text { shRNA in human cells }\end{array}$ & $\begin{array}{l}\text { Kindly provided by Prof. } \\
\text { Matthias Dobbelstein } \\
\text { (Göttingen, Germany) }\end{array}$ \\
\hline
\end{tabular}




\begin{tabular}{lll} 
Vector & Purpose & Reference \\
\hline \hline pRcCMV-p53-R175H- & $\begin{array}{l}\text { CMV-promotor driven } \\
\text { 72R }\end{array}$ & $\begin{array}{l}\text { Kindly provided by Prof. } \\
\text { human cells }\end{array}$ \\
\hline
\end{tabular}

\subsubsection{Human Cell Lines}

Table 2.10 provides the used human cell lines, their origin and references.

Table 2.10: Human Cell Lines

\begin{tabular}{|c|c|c|}
\hline Cell Line & Origin & Reference \\
\hline HCT116 & colon carcinoma & Brattain et al. 1981 \\
\hline SW48 & colon carcinoma & Leibovitz et al. 1976 \\
\hline SW620 & colon carcinoma & Leibovitz et al. 1976 \\
\hline SW480 & colon carcinoma & Leibovitz et al. 1976 \\
\hline SW837 & rectum carcinoma & Leibovitz et al. 1976 \\
\hline $\mathrm{CaCo} 2$ & colon carcinoma & Heinen et al. 1995 \\
\hline HT29 & colon carcinoma & Adachi et al. 1987 \\
\hline LS411N & colon carcinoma & Suardet et al. 1992 \\
\hline LS513 & colon carcinoma & Suardet et al. 1992 \\
\hline Colo201 & colon carcinoma & Semple et al. 1978 \\
\hline LoVo & colon carcinoma & Drewinko et al. 1976 \\
\hline HCT116-TP53 ${ }^{/-}$ & colon carcinoma & Langermann 1998 \\
\hline HCT116-TP73 shRNA & colon carcinoma & $\begin{array}{l}\text { kindly provided by Dr. Daniela } \\
\text { Kramer (Göttingen, Germany) }\end{array}$ \\
\hline RKO & colon carcinoma & Brattain et al. 1984 \\
\hline RKO CDKN1A inducible & colon carcinoma & Schmidt et al. 2000 \\
\hline SK-Mel-19 & melanoma & Carey et al. 1976 \\
\hline SK-Mel-28 & melanoma & Carey et al. 1976 \\
\hline
\end{tabular}




\begin{tabular}{lll} 
Cell Line & Origin & Reference \\
\hline \hline SK-Mel-103 & melanoma & Gruis et al. 1995 \\
\hline SK-Mel-147 & melanoma & Gruis et al. 1995
\end{tabular}

\section{Generated Human Cell Lines}

The following table lists the cell lines that were generated during this study.

Table 2.11: Generated Human Cell Lines

\begin{tabular}{|c|c|c|c|}
\hline Cell line & Parental cell line & Plasmid & $\begin{array}{l}\text { Selection } \\
\text { Marker }\end{array}$ \\
\hline HCT116-TP53 $3^{--}$- ctr & HCT116 TP53 ${ }^{-/-}$ & pRetroSuper-scrambled & Puromycin \\
\hline $\begin{array}{l}\text { HCT116-TP53 }{ }^{-/}- \\
\text {TP73 shRNA }\end{array}$ & HCT116 TP53 ${ }^{-/-}$ & pLKO.1-sh-TP73 & Puromycin \\
\hline HCT116- ctr & HCT116 & plnducer20 & Puromycin \\
\hline HCT116- TAp73a & HCT116 & plnducer20-TAp73a & Puromycin \\
\hline HCT116-TP53 ${ }^{-/}-\mathrm{ctr}$ & HCT116 TP53 ${ }^{-/}$ & plnducer20 & Puromycin \\
\hline 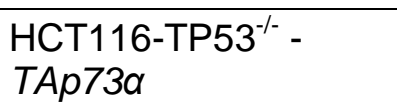 & HCT116 TP53 $^{-/-}$ & plnducer20-TAp73a & Puromycin \\
\hline $\begin{array}{l}\text { HCT116-TP53 }{ }^{--}- \\
\text {TP73 shRNA- ctr }\end{array}$ & HCT116 TP53 ${ }^{-/-}$ & plnducer20 & Puromycin \\
\hline $\begin{array}{l}\text { HCT116-TP53 }^{-/}- \\
\text {TP73 shRNA-TAp73a }\end{array}$ & HCT116 TP53\% & plnducer20-TAp73a & Puromycin \\
\hline $\begin{array}{l}\text { HCT116-p53-R175H- } \\
\text { ctr }\end{array}$ & HCT116 p53 R175H & pRetroSuper-scrambled & Puromycin \\
\hline $\begin{array}{l}\text { HCT116-p53-R175H- } \\
\text { TP73 shRNA }\end{array}$ & HCT116 p53 R175H & pRcCMV-p53-R175H-72R & Puromycin \\
\hline
\end{tabular}

\section{Cultivation of Human Cells}

Human cells were cultivated at $37^{\circ} \mathrm{C}$ and $5 \% \mathrm{CO}_{2}$. Cells were cultured in RPMI medium (Sigma-Aldrich, Taufkirchen, Germany) supplemented with 10\% FCS (GE Healthcare, Chalfont St. Giles, Great Britain), $100 \mu \mathrm{g} / \mathrm{ml}$ streptomycin and $100 \mathrm{U} / \mathrm{ml}$ penicillin (SigmaAldrich, Taufkirchen, Germany). Cells were passaged three times a week. For this, cells were washed once with PBS and detached by using trypsin (Sigma-Aldrich, Taufkirchen, Germany). A defined amount of cell suspension was transferred to a new cell culture dish. 
On a long term basis, cells were stored in liquid nitrogen. Therefore, cells were harvested and resuspended in freezing medium (70\% RPMI, 20\% FCS and 10\% DMSO), slowly cooled to $-80^{\circ} \mathrm{C}$ using a cryo $1^{\circ} \mathrm{C}$ freezing container and transferred to the vapor phase of liquid nitrogen.

\subsection{Cell Biological Methods}

\subsubsection{Transfection Of Human Cells}

\section{Transfection Using siRNA}

Cells were seeded at $70 \%$ density in 6 -well plates $24 \mathrm{~h}$ prior to transfection. siRNA transfections were performed by using INTERFERin reagent (Polyplus, Illkirch, Frankreich). $60 \mathrm{pmol}$ siRNA were diluted in $189 \mu \mathrm{l}$ serum free medium. $5 \mu \mathrm{l}$ INTERFERin were added and the mixture was vortexed for $10 \mathrm{~s}$. After incubating for $10 \mathrm{~min}$ at room temperature the mixture was dropped onto the cells. The medium was changed after $4 \mathrm{~h}$ and the knock down of respective targets was checked $48 \mathrm{~h}$ after transfection.

\section{Plasmid Transfection Using Electroporation}

Cells were harvested by centrifugation at $1000 \mathrm{rpm}$ for $5 \mathrm{~min}$ and resuspended in a concentration of $5 \times 10^{6}$ cells $/ \mathrm{ml}$ in RPMI medium. $10-50 \mu \mathrm{g}$ of plasmid DNA was added to 400 $\mu \mathrm{l}$ cell suspension. Electroporation was performed in a $4 \mathrm{~mm}$-cuvette at $300 \mathrm{~V}$ and $500 \mu \mathrm{F}$ (HCT116, SW48, RKO, SW480, SW620, CaCo2, HT29), $220 \mathrm{~V}$ and $950 \mu \mathrm{F}$ (SW837, LS411N, LS513, Colo201, LoVo) or $200 \mathrm{~V}$ and 500 HF (SK-Mel-19, SK-Mel-28, SK-Mel-103, SK-Mel-147). Cells were transferred into RPMI medium. After $6 \mathrm{~h}$, cells were washed once with PBS and supplemented with fresh RPMI medium. Analyses were performed after $48 \mathrm{~h}$.

\section{Plasmid Transfection Using Polyethylenimin (PEI)}

Cells were seeded at $70 \%$ density in 6 -well plates $24 \mathrm{~h}$ prior to transfection. Before transfection, the cells were washed with PBS and supplemented with fresh culture medium. For transfection, two solutions were prepared: solution A contained $100 \mu$ PBS mixed with 2$7 \mu \mathrm{g}$ plasmid DNA, whereas solution B contained $95 \mu \mathrm{l}$ PBS mixed with $5 \mu \mathrm{IPEI}(0.0025 \%$ final concentration). Both solutions were mixed by pipetting and were incubated at room temperature for $15 \mathrm{~min}$. The mixture was dropped onto the cells and after $4 \mathrm{~h}$ cells were washed three times and supplemented with fresh RPMI medium. Analyses were performed after $48 \mathrm{~h}$. 


\section{Plasmid Transfection Using METAFECTENE ${ }^{\odot}$}

Cells were seeded at $70 \%$ density in 6 -well plates $24 \mathrm{~h}$ prior to transfection. For the generation of stable expression cell lines, METAFECTENE ${ }^{\odot}$ (Biontex Laboratories $\mathrm{GmbH}$, Martinsried, Germany) was used. Two solutions were prepared: solution A contained $100 \mu \mathrm{l}$ PBS mixed with $2 \mu \mathrm{g}$ plasmid DNA, solution B contained $100 \mu \mathrm{l}$ PBS mixed with $6 \mu \mathrm{l}$ METAFECTENE ${ }^{\odot}$. Both solutions were mixed and incubated at room temperature for $15 \mathrm{~min}$. Afterwards, the mixture was dropped onto the cells. $6 \mathrm{~h}$ after transfection cells were washed twice with PBS and supplemented with fresh cell culture medium.

\subsubsection{Generation Of Stable Cell Lines}

For the generation of cell lines stably expressing plasmid DNA cells were transfected with METAFECTENE $^{\odot} .24 \mathrm{~h}$ after transfection, cells were seeded in different dilutions (1:250, $1: 500,1: 1000,1: 5000)$ into RPMI medium containing $1 \mu \mathrm{g} / \mathrm{ml}$ puromycin. Puromycin enables the selection of successfully transfected cells. Analyses of the single cell clones were performed after 30 generations.

\subsubsection{Karyotype Analyses}

\section{Chromosome Spreading}

Cells were treated with $150 \mathrm{nM}$ Taxol for $4 \mathrm{~h}$. After centrifugation at $2000 \mathrm{rpm}$ for $5 \mathrm{~min}$, cells were resuspended in $2 \mathrm{ml}$ hypotonic solution (40\% RPMI medium, 60\% aqua dest.) and incubated for $20 \mathrm{~min}$ at room temperature. Afterwards, cells were fixed with $1 \mathrm{ml}$ ice-cold Carnoy's fixative (75\% methanol, 25\% glacial acetic acid) and centrifuged for $5 \mathrm{~min}$ at 2000 rpm. The fixation step was repeated three times. Subsequently, cells were resuspended in $500 \mu \mathrm{l} 100 \%$ glacial acetic acid and dropped onto a pre-cooled, wet object slide, which was in turn incubated for $5 \mathrm{~min}$ at $42^{\circ} \mathrm{C}$. Dried slides were stained in $8 \%$ Giemsa staining solution (Merck, Darmstadt, Germany) in $\mathrm{H}_{2} \mathrm{O}$ for 25 min. Slides were washed and dried at room temperature and embedded in Euparal (Carl Roth, Karlsruhe, Germany). Object slides were analyzed using Zeiss Axioscope FS microscope (Zeiss, Oberkochen, Germany) equipped with a Hamamatsu C4742-95 camera and the Hokawo Launcher 2.1 software (Hamamatsu Photonics, Hamamatsu, Japan)

\section{CEP-FISH}

Asynchronously growing cells were harvested by centrifugation at $2000 \mathrm{rpm}$ for $5 \mathrm{~min}$, resuspended in hypotonic solution, fixed by using Carnoy's fixative and dropped onto coverslips as described above. 
Object slides were incubated in 2x SCC solution (0.3 M sodium chloride, $30 \mathrm{mM}$ trisodium citrate, $\mathrm{pH} 7.0$ ) for $2 \mathrm{~min}$ at room temperature. After dehydration in an alcohol series of $70 \%$, $85 \%$ and $100 \%$ ethanol for 2 min each, object slides were air dried. $0.6 \mu$ l of each probe specific for chromosome 7 and chromosome 15 or chromosome 6 and chromosome 18 were added to the hybridization solution and pre-warmed for $5 \mathrm{~min}$ at $37^{\circ} \mathrm{C}$. Cells were covered with the probe mixture. Sample and probe were heated for 2 min at $75^{\circ} \mathrm{C}$ and placed for $2 \mathrm{~h}$ at $37^{\circ} \mathrm{C}$. Afterwards, the cover slip was immersed in $0.25 x$ SSC at $72^{\circ} \mathrm{C}$ for $4 \min (\mathrm{CRC}$ cell lines) or $6 \mathrm{~min}$ (melanoma cell lines). Thereafter, the cells were incubated in 2x SSC containing $0.05 \%$ Tween-20 at room temperature for $5 \mathrm{~min}$ and stained with Hoechst 33342 in $2 x$ SSC $+0.05 \%$ Tween-20 for 5 min. Object slides were washed three times with 2x SSC $+0.05 \%$ Tween-20, dried and embedded in Vectashield (Vector Laboratories, Burlingame, CA, USA). Analyses were performed on a DeltaVision Elite microscope.

\subsubsection{Synchronization Of Human Cells}

\section{Cell Cycle Synchronization via Double Thymidine Block}

Cell cycle synchronization of human cells at the $\mathrm{G}_{1}$-/S-transition was achieved by a double thymidine block. Cells were grown in $2 \mathrm{mM}$ thymidine for $16 \mathrm{~h}$. After washing the cells five times with PBS, they were released into fresh culture medium for $8 \mathrm{~h}$. Following, cells were seeded in $2 \mathrm{mM}$ thymidine for another $16 \mathrm{~h}$. Cells were washed five times with PBS and released into fresh culture medium. For the analysis of lagging chromosomes, cells were fixed $8.5 \mathrm{~h}$ ( $\mathrm{CRC}$ cell lines) or $9.5 \mathrm{~h}$ (melanoma cell lines) after release from thymidine.

\section{Mitotic Synchronization}

Synchronization of cells in mitosis was achieved by treatment with $2 \mu \mathrm{M}$ DME for $16 \mathrm{~h}$.

\subsubsection{Immunofluorescence Microscopy}

Cells were fixed for immunofluorescence microscopy by using $2 \%$ paraformaldehyde in PHEM buffer (60 mM PIPES pH 7.0, 27 mM HEPES, $10 \mathrm{mM} \mathrm{EGTA,} 4 \mathrm{mM} \mathrm{MgCl}$ ) for $5 \mathrm{~min}$ at room temperature followed by 5 min methanol fixation at $-20^{\circ} \mathrm{C}$. In order to avoid unspecific antibody binding, cells were blocked with 5\% FCS in PBS for $30 \mathrm{~min}$. Afterwards, cells were incubated with primary antibodies in PBS containing $2 \%$ FCS for $1.5 \mathrm{~h}$ at room temperature. Afterwards, cells were washed three times with PBS and incubated with fluorescence-labeled secondary antibody for $1.5 \mathrm{~h}$ at room temperature. After washing once with PBS, cells were incubated in Hoechst33342 (1:15000 in PBS) (Invitrogen, Carlsbad, CA, USA) for $5 \mathrm{~min}$ and washed three times with PBS. Object slides were dried and embedded 
using VectaShield (Vector Laboratories, Burlingame, CA, USA). Cells were analyzed using a Leica DM600B fluorescence microscope equipped with an ORCA-ER camera. Images were taken in a 60x magnification with a z-optical spacing of $0.2 \mu \mathrm{m}$. Deconvolution and further analyses were performed using the Leica LAS-AF software. Alternatively, a DeltaVision Elite microscope (GE Healthcare, Chalfont St. Giles, Great Britain) equipped with a PCO Edge sCMOS camera (PCO, Kelheim, Germany) was used. Images were taken with an Olympus 60x 1.40 NA objective (Olympus, Tokio, Japan) with a z-optical spacing of $0.4 \mu \mathrm{m}$, deconvolved and further analyzed using the Softworx 5.0 software.

\subsubsection{Analyses Of Spindle Orientation}

Asynchronously growing cells were seeded onto fibronectin coated coverslips (BD Biosciences, Heidelberg, Germany). Cells were fixed using ice cold methanol for $6 \mathrm{~min}$ at $-20^{\circ} \mathrm{C}$, blocked by using 5\% FCS in PBS for $20 \mathrm{~min}$ and stained for $\alpha$-tubulin, $\mathrm{\gamma}$-tubulin and DNA. Microscopy was performed on a DeltaVision-ELITE microscope (GE Healthcare, Chalfont St. Giles, Great Britain) equipped with a PCO Edge sCMOS camera (PCO, Kelheim, Germany). Images were acquired with an Olympus 60x 1.40 NA objective (Olympus, Tokio, Japan) with a z-optical spacing of $0.4 \mu \mathrm{m}$, deconvolved and analyzed using SoftWorx 5.0 software (Applied Precision Inc., Issaquah, WA, USA). Determination of spindle orientation was calculated using the following formula: spindle axis angle $=$ (number of $z-$ stacks ${ }^{*} 0.4 \mu \mathrm{m} /$ distance of centrosomes) ${ }^{*} 180 / \pi$.

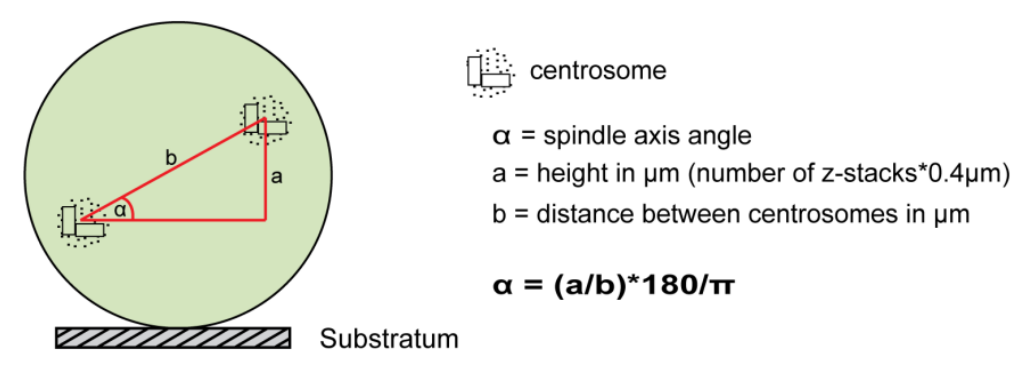

Fig. 2.1: Schematic illustration for the determination of the spindle axis angle.

\subsubsection{Analyses Of Microtubule Plus-End Assembly Rates}

Cells were transfected with $10 \mu \mathrm{g}$ pEGFP-EB3 plasmid via electroporation $48 \mathrm{~h}$ prior to live cell analysis. For analysis of microtubule polymerization rates of monopolar spindles, cells were treated with $2 \mu \mathrm{M}$ DME in phenol red free RPMI medium for $1 \mathrm{~h}$ (CRC cell lines) $-2 \mathrm{~h}$ (melanoma cell lines). Live cell analyses were performed at $37^{\circ} \mathrm{C}$ and $5 \% \mathrm{CO}_{2}$ using a Delta Vision Elite microscope equipped with a PCO edge sCMOS camera. Images of 4 sections 
with a z-optical spacing of $0.4 \mu \mathrm{m}$ were taken every $2 \mathrm{sec}$ for $30 \mathrm{sec}$. Images were deconvolved and further analyzed using the Soft WoRX 5.0 software.

\subsubsection{Chorion-Allantoic Membrane (CAM) Assay}

Fertilized White Leghorn chick eggs were incubated at $37.8^{\circ} \mathrm{C}$ and $80 \%$ relative humidity. A small window was cut into the shell on day three of chick development. Eggs with resealed window were further incubated until day 10 of chick development. Two million HCT116 cells per CAM were resuspended in a mixture of 50\% RPMl1640 medium and 50\% matrigel in a total volume of $40 \mu \mathrm{l}$. After incubation of cell suspensions for $30 \mathrm{~min}$ at $37^{\circ} \mathrm{C}$ and $5 \% \mathrm{CO}_{2}$, cells were applied on top of the CAM. On day 17 of chick development, tumors were dissected.

\subsubsection{Immunohistology}

For immunohistological analyses, dissected tumors were fixed in $4 \%$ paraformaldehyde for $20 \mathrm{~min}$, washed three times with PBS and transferred into $10 \%$ sucrose for $2 \mathrm{~h}$ at $4^{\circ} \mathrm{C}$. After an overnight incubation in $30 \%$ sucrose at $4^{\circ} \mathrm{C}$ tumors were embedded in tissue freezing medium and stored at $-80^{\circ} \mathrm{C}$. Hardened tumor blocks were cut in $12 \mu \mathrm{m}$ thick sections using a cryotome. Cryosections were transferred onto microscope slides and stored at $-20^{\circ} \mathrm{C}$.

For staining of the cryosections, microscope slides were thawed, dried at room temperature and blocked using blocking solution (1\% BSA, 5\% goat serum and $0.2 \%$ Triton $\mathrm{X}-100$ in PBS) for $30 \mathrm{~min}$. Afterwards, sections were covered with primary antibodies diluted in antibody solution ( $1 \% \mathrm{BSA}$ and $0.5 \%$ Triton X-100 in $0.05 \mathrm{M} \mathrm{TBS}$ ) over night at $4^{\circ} \mathrm{C}$. The cryosections were washed two times with PBS. Subsequently, they were covered with secondary antibody and DAPI mixed in antibody solution for $1 \mathrm{~h}$ at room temperature. Specimens were washed three times, mounted in Fluoromount-G (Sigma-Aldrich, Taufkirchen, Germany) and analyzed with a Zeiss Axio Imager Z1 microscope (Carl Zeiss, Göttingen, Germany).

\subsubsection{In Vitro Migration Assay}

Cells were starved for $16 \mathrm{~h}$ in medium containing 0.5\% FCS. Cells were harvested, counted and resuspended in medium containing $0.5 \%$ FCS to a final cell concentration of $2 * 10^{5}$ cells per $200 \mu \mathrm{l}$. ThinCert ${ }^{\odot}$ cell culture inserts (Greiner BioOne, Frickenhausen, Germany) were placed in a 24-well plate containing $600 \mu$ culture medium including $10 \%$ FCS. The cell suspension was added into the cell culture inserts. Cells were incubated for $24 \mathrm{~h}$ at $37^{\circ} \mathrm{C}$ and $5 \% \mathrm{CO}_{2}$ before being detached from the bottom of the cell culture insert by Trypsin/EDTA. Detached cells were resuspended in $100 \mu \mathrm{PBS}$ and counted. 


\subsubsection{In Vitro Invasion Assay}

Matrigel coated cell culture inserts (BD Bioscience, Bedford, MA, USA) were rehydrated according to the manufacturer's protocol. Meanwhile, cells were harvested, counted and resuspended in cell culture medium containing $0.5 \%$ FCS to a final concentration of $2 * 10^{5}$ cells per $500 \mu \mathrm{l}$. Cell culture inserts were placed in a 24 -well containing $750 \mu \mathrm{m}$ cell culture medium. The cell suspension was added into the cell culture inserts and incubated for $48 \mathrm{~h}$ at $37^{\circ} \mathrm{C}$ and $5 \% \mathrm{CO}_{2}$. Following, non-invasive cells were removed from the upper surface of the membrane by scrubbing using a moistened cotton swab. Cells on the lower surface of the membrane were fixed using 2\% PFA for $5 \mathrm{~min}$ at room temperature and methanol for 5 min at $-20^{\circ} \mathrm{C}$. Cells were stained using Hoechst33342 for $5 \mathrm{~min}$. After the membrane was dried, it was cut from the insert, placed on an object slide and embedded using VectaShield ${ }^{\odot}$. Invasive cells were analyzed under the microscope at 40x magnification. Ten randomly chosen pictures were taken for each assay. Cells were counted using the ImageJ Software.

\subsubsection{Rac1-Activation-Assay}

Rac1-Activation-Assay was performed using the RhoA/Rac1/Cdc42 Activation Combo Biochem Kit (Cytoskeleton Inc, Denver, CO, USA) according to the manufacturer's protocol. Cell culture plates were placed on ice and the cells were washed once with ice cold PBS. Cell lysis was performed on the plate by using $400 \mu$ ice-cold lysis buffer (50 mM Tris pH $7.5,10 \mathrm{mM} \mathrm{MgCl}_{2}, 0.5 \mathrm{M} \mathrm{NaCl}, 2 \%$ Igepal). Cells were harvested and centrifuged for $1 \mathrm{~min}$ at $14800 \mathrm{rpm}$ at $4^{\circ} \mathrm{C}$. The supernatant was transferred to a new reaction tube and snap frozen in liquid nitrogen. $1500 \mu \mathrm{g}$ of cell lysate were incubated with $10 \mu \mathrm{g}$ PAK-PBD-beads for 30 min at $4^{\circ} \mathrm{C}$. Beads were washed once with wash buffer (25 mM Tris pH 7.5, $30 \mathrm{mM} \mathrm{MgCl}_{2}$, $40 \mathrm{mM} \mathrm{NaCl}$ ) and resuspended in $15 \mu \mathrm{l}$ Laemmli sample buffer. Samples were boiled for 5 min and analyzed by SDS-PAGE and western blot. Rac1 was detected using anti-Rac1 antibody (Rac1 Arc03, Cytoskeleton Inc., Denver, CO, USA) at 1:500 dilution.

\subsubsection{GEF-Assay}

GEF-Assay for TRIO was performed by using Rac1 G15A Agarose Beads (Cell Biolabs Inc., San Diego, CA, USA). Cell culture plates were placed on ice and the cells were washed once with ice cold PBS. Cell lysis was performed on the plate using $400 \mu \mathrm{l}$ ice-cold lysis buffer (20 mM HEPES, pH 7.5, $150 \mathrm{mM} \mathrm{NaCl}, 5 \mathrm{mM} \mathrm{MgCl} 2,1 \%$ Triton X-100). Cells were harvested and centrifuged for $1 \mathrm{~min}$ at $14800 \mathrm{rpm}$ at $4^{\circ} \mathrm{C}$. $2000 \mu \mathrm{g}$ of cell lysate were incubated with 10 $\mu \mathrm{g}$ Rac1 G15A agarose beads for $1 \mathrm{~h}$ at $4^{\circ} \mathrm{C}$. Beads were washed three times with HBS buffer (20 mM HEPES, pH 7.5, $150 \mathrm{mM} \mathrm{NaCl}$ ) and resuspended in $15 \mu$ Laemmli sample buffer. Samples were boiled for $5 \mathrm{~min}$ and analyzed by SDS-PAGE and western blot. TRIO 
was detected using anti-TRIO antibody (TRIO D20, Santa Cruz, Dallas, TX, USA) at 1:300 dilution.

\subsection{Proteinbiochemistry}

\subsubsection{Preparation Of Protein Lysates}

Cells were harvested by centrifugation at $2000 \mathrm{rpm}$ for $5 \mathrm{~min}$ and subsequently resuspended in $70 \mu$ lysis Buffer (50 mM Tris- $\mathrm{HCl}$ pH 7.4, $150 \mathrm{mM} \mathrm{NaCl,} 5$ mM EDTA, 5 mM EGTA, 1\% $(\mathrm{v} / \mathrm{v})$ Igepal $^{\odot}, 0.1 \%(\mathrm{w} / \mathrm{v})$ SDS, $0.1 \%$ Na-Desoxycholate, $20 \mathrm{mM} \mathrm{Na}_{3} \mathrm{VO}_{4}, 25 \mathrm{mM} \beta-$ Glycerophosphate, $50 \mathrm{mM} \mathrm{NaF}, 5 \mathrm{mM} \mathrm{Na}_{2} \mathrm{MoO}_{4}$ ) supplemented with complete protease inhibitor cocktail EDTA-free (1:25) (Roche, Switzerland), phosphatase inhibitor (1:10) and 0.5 $\mu \mathrm{M}$ microcystin-LR (Enzo Life Sciences, Lörrach, Germany). After 10 min incubation on ice, cells were centrifuged at $14800 \mathrm{rpm}$ for $20 \mathrm{~min}$ at $4^{\circ} \mathrm{C}$. The supernatant was transferred to a new reaction tube and stored at $-20^{\circ} \mathrm{C}$.

\subsubsection{Protein Determination}

Protein levels were determined by using the Bio-Rad DC Protein assay (BioRad, Hercules, CA, USA) following the manufacturer's protocol. Photometric measurement was performed using a VICTOR $^{\odot}$ X3 microplate reader.

\subsubsection{Sodiumdodecylsulfate-Polyacrylamid Gel Electrophoresis (SDS-PAGE)}

A discontinuous SDS-PAGE was used for the separation of proteins according to their molecular weight. The SDS-gel composed of a $5 \%$ stacking gel $(300 \mathrm{nM}$ TRIS-HCl pH 6.8, $0.1 \%(\mathrm{w} / \mathrm{v})$ SDS, $5 \%(\mathrm{v} / \mathrm{v})$ Rotiphorese $^{\odot}$ Gel 30 ) and a $6-13 \%$ resolving gel (500 mM TRIS$\mathrm{HCl} \mathrm{pH} \mathrm{8.8,} \mathrm{0.1 \%} \mathrm{(w/v)} \mathrm{SDS,} \mathrm{6-13 \%} \mathrm{(v/v)} \mathrm{Rotiphorese}{ }^{\odot}$ Gel 30). $5 \mu$ l of PageRuler Prestained Protein Ladder (Fermentas, St. Leon-Rot, Germany) was used for the determination of the molecular weight of the detected proteins. $50 \mu \mathrm{g}$ protein extract were supplemented with SDS sample buffer (15\% (w/v) SDS, 15\% (v/v) $\beta$-mercaptoethanol, 50\% glycerol, $0.25 \%$ $(\mathrm{w} / \mathrm{v})$ bromophenol blue). For protein denaturation, samples were heated at $95^{\circ} \mathrm{C}$ for $5 \mathrm{~min}$. Proteins were separated in SDS running buffer $(25 \mathrm{mM}$ TRIS- $\mathrm{HCl}$ pH 6.8, $192 \mathrm{mM}$ glycine, $0.15 \%(w / v)$ SDS ) for $1 \mathrm{~h}$ at $28 \mathrm{~mA}$ and $2.5-3.5 \mathrm{~h}$ at $38 \mathrm{~mA}$. 


\subsubsection{Western Blot}

\section{Semi-dry Blot}

After separation of proteins via SDS-PAGE, they were transferred onto a nitrocellulose membrane by semi dry western blot. Transfer was performed in a blotting device at $200 \mathrm{~mA}$ for $1.5 \mathrm{~h}$ using transfer buffer $(0.0025 \%$ (w/v) SDS, $24.8 \mathrm{mM}$ TRIS-HCl pH 8.0, $170 \mathrm{mM}(\mathrm{v} / \mathrm{v})$ glycine, $20 \%$ methanol).

\section{Tank-Blotting}

Proteins larger than $100 \mathrm{kDa}$ were transferred onto nitrocellulose membrane via tank blotting. Transfer was performed in a tank blot device for $3 \mathrm{~h}$ at $400 \mathrm{mM}$ by using blotting buffer (0.0025\% (w/v) SDS, $24.8 \mathrm{mM}$ TRIS-HCl pH 8.0, $170 \mathrm{mM}(\mathrm{v} / \mathrm{v})$ glycine, 13\% methanol).

After protein transfer the membrane was blocked for 30 min using $5 \%$ milk powder in TBS (50mM TRIS- $\mathrm{HCl}$ pH 7.2, $160 \mathrm{mM} \mathrm{NaCl}$ ). The proteins of interest were detected with specific primary antibodies diluted in $2 \%$ BSA in TBS. The primary antibody was incubated over night at $4^{\circ}$. After washing the membrane three times using TBS-T $(0.1 \%$ Tween in TBS) it was incubated with the secondary antibody conjugated to HRP for $1 \mathrm{~h}$ at room temperature. Subsequently, the membrane was washed three times in TBS-T and once in TBS for $10 \mathrm{~min}$ each. Proteins were detected by ECL. For this, the membrane was incubated shortly in $0.1 \mathrm{mM}$ TRIS- $\mathrm{HCl} \mathrm{pH} 8.5$ containing $0.4 \mathrm{mM} \beta$-coumaric acid, $2.5 \mathrm{mM}$ luminol and $0.03 \%$ $\mathrm{H}_{2} \mathrm{O}_{2}$. Chemoluminescence was detected using Fuji Medical X-Ray Film and the OPTIMAX$\mathrm{X}$-Ray Film Processor.

\subsection{Molecular Biological Methods}

\subsubsection{Escherichia coli cells}

The following E. coli strain was used:

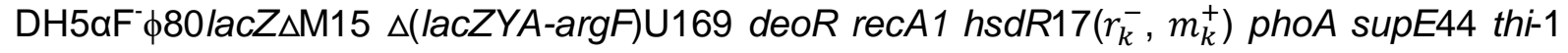
gyrA96 relA1^-

\subsubsection{Generation Of Competent Escherichia coli Cells}

E. coli DH5a were inoculated in $5 \mathrm{ml}$ Luria Bertani medium (LB medium) (1\% peptone 140, $0.5 \%$ yeast extract, $0.5 \% \mathrm{NaCl}$ ) and kept at $37^{\circ} \mathrm{C}$ overnight on a shaker. The preculture was transferred to $400 \mathrm{ml} \mathrm{LB}$ medium and grown to a density of $\mathrm{OD}_{600}=0.5$ at $37^{\circ} \mathrm{C}$. The culture 
was incubated on ice for $5 \mathrm{~min}$. After centrifugation for $5 \mathrm{~min}$ at $2000 \mathrm{rpm}$ and resuspending in $40 \mathrm{ml}$ cold $\mathrm{Tfbl}$ buffer ( $30 \mathrm{mM}$ potassium acetate, $100 \mathrm{mM} \mathrm{RbCl}, 10 \mathrm{mM} \mathrm{CaCl}, 15 \%$ glycerol, $\mathrm{pH}$ adjusted to 6.0 using $0.2 \mathrm{M}$ acetic acid containing $50 \mathrm{mM}$ of $\mathrm{MnCl}_{2}$ ) the suspension was again incubated on ice for $5 \mathrm{~min}$ and centrifuged for $5 \mathrm{~min}$ at $2000 \mathrm{rpm}$. After resuspending in $4 \mathrm{ml}$ cold Tfbll buffer (10 mM MOPS, $75 \mathrm{mM} \mathrm{CaCl}_{2}, 10 \mathrm{mM} \mathrm{RbCl}, 15 \%$ glycerol, $\mathrm{pH} 6.5$ ) the cells were incubated on ice for $15 \mathrm{~min}$. Aliquots of $100 \mu \mathrm{l}$ were snap frozen in liquid nitrogen and stored at $-80^{\circ} \mathrm{C}$ until usage.

\subsubsection{Transformation Of Escherichia coli Cells}

Transformation of $E$. coli was performed using the heat-shock protocol. $50 \mu$ l of competent $E$. coli DH5a cells were mixed with $2 \mu \mathrm{g}$ plasmid DNA and incubated on ice for $20 \mathrm{~min}$. Subsequently, cells were incubated for $40 \mathrm{sec}$ at $42^{\circ} \mathrm{C}$ followed by an incubation on ice for 2 min. Cells were supplemented with $600 \mu \mathrm{LB}$ medium and incubated for 60 min at $37^{\circ} \mathrm{C}$ while constant shaking. Cells were transferred to $400 \mathrm{ml}$ LB medium containing appropriate antibiotics for selection of the plasmid. After overnight incubation at $37^{\circ} \mathrm{C}$ plasmid DNA was isolated from the liquid culture.

\subsubsection{Plasmid Isolation}

Plasmid DNA preparation was performed using the NucleoBond ${ }^{\odot}$ PC 100 X-TRA Midi Kit (Macherey Nagel, Düren, Germany) according to the manufacturer's protocol.

The DNA concentration was measured using the NanoDrop ${ }^{\odot} 2000$ spectro-photometer (ThermoFisher, Waltham, MA, USA).

\subsubsection{RNA-Isolation}

RNA was isolated using the NucleoSpin ${ }^{\odot}$ RNA Kit according to the manufacturer's protocol (Macherey Nagel, Düren, Germany).

RNA was quantified using NanoDrop ${ }^{\odot} 2000$ spectrophotometer (ThermoFisher, Waltham, MA, USA) and analysed in a $1 \%$ agarose gel.

\subsection{6 cDNA Synthesis And Quantitative Real Time PCR}

$1 \mu \mathrm{g}$ cDNA was synthesized from RNA using 100 pmol random hexamer primer, dNTPs (0.5 mM each), $20 \cup$ RiboLock RNase inhibitor and $200 \mathrm{U}$ Maxima Reverse Transcriptase (ThermoFisher, Waltham, MA, USA). Reaction components were incubated for $10 \mathrm{~min}$ at $25^{\circ} \mathrm{C}$ followed by $30 \mathrm{~min}$ at $50^{\circ} \mathrm{C}$. The reaction was terminated by heating at $85^{\circ} \mathrm{C}$ for $5 \mathrm{~min}$. Quantitative real time PCR reactions were performed using gene specific primers, $30 \mathrm{ng}$ 
cDNA and GoTaq qPCR Master Mix (Promega, Mannheim, Germany) on a Rotor Gene Q real time PCR cycler (Qiagen, Hilden, Germany) using the following program (Table 2.12).

Table 2.12: qRT-PCR Program

\begin{tabular}{lll}
\hline Denaturation & $95^{\circ} \mathrm{C} 5 \mathrm{~min}$ & \\
\hline Denaturation & $95^{\circ} \mathrm{C} 5 \mathrm{~s}$ & \\
& & \\
\hline Primer Annealing & $60^{\circ} \mathrm{C} 10 \mathrm{~s}$ & \\
& & \\
\hline Polymerization & $60^{\circ} \mathrm{C} 10 \mathrm{~s}$ & \\
\hline
\end{tabular}

Transcriptional changes were calculated based on the comparative $\mathrm{C}_{\mathrm{T}}$ method.

\subsubsection{Preparation of Samples For RNA-Sequencing}

HCT116 cells were seeded in $2 \mathrm{mM}$ Thymidine for $16 \mathrm{~h}$, released into the cell cycle for $8 \mathrm{~h}$ and seeded into $2 \mathrm{mM}$ Thymidine. After $1 \mathrm{~h}$, siRNA transfections were conducted using 60 pmol LUCIFERASE, TP53 or TP73 siRNA. Medium was changed after $2 \mathrm{~h}$. After $11 \mathrm{~h}$, cells were released into the cell cycle for $6.5 \mathrm{~h}$ and subsequently harvested. These $\mathrm{G}_{2}$ arrested probes were further processed by the Transcriptome and Genome Analysis Laboratory (TAL) of the University of Göttingen (Göttingen, Germany). Bioinformatical analyses were performed by Silvia von der Heyde (former University of Göttingen, Germany). 


\section{Results}

\subsection{A Mechanistic Link Between Chromosomal Instability And Tumor Cell Migration}

\subsubsection{Increased Interphase Microtubule Plus-End Assembly Rates Correlate With Migration And Invasion}

Aneuploidy is a hallmark of human cancer, which derives from an increased rate of chromosome missegregation during mitosis. This process, termed chromosomal instability $(\mathrm{CIN}$ ), contributes to tumorigenesis, tumor progression and therapy resistance (Holland \& Cleveland 2012). However, the mechanisms behind are not well understood. CIN is often associated with late tumor stages, which are associated with larger tumors, high invasiveness and infiltration of nearby tissues. Thus, we wanted to investigate a possible link between CIN and cancer cell migration and invasion.

To investigate a potential correlation between these two phenotypes, transwell migration and invasion assays were performed. A panel of CRC cells was used, which can be subdivided into chromosomally stable, but microsatellite instable (MIN/MSI) cell lines (HCT116, SW48) and chromosomally instable (CIN) cell lines (SW480, SW620, SW837, CaCo2, HT29, LS411N, LS513, Colo201, LoVo). Intriguingly, only the CIN cell lines Colo201 and LoVo exhibited increased migration and invasion (Fig 3.1a-b). In comparison to HCT116 cells, Colo201 and LoVo cells displayed an approximal 2-fold increased migration potential as well as a nearly 2-fold increased invasion potential. SW620, SW837, CaCo2, HT29, LS411N and LS513 exhibited the lowest migration and invasion potentials. Increased microtubule plusend assembly rates in mitosis were recently elucidated as a common cause for CIN in colorectal cancer (Ertych et al. 2014). Microtubule plus-end assembly rates were measured during mitosis in the panel of CRC cell lines. The measurement was performed using the GFP-tagged end binding protein EB3, which binds to growing microtubule plus-ends (Stepanova et al. 2003). Thus, by using live cell microscopy, the growing microtubule plusends can be tracked over time and microtubule plus-end assembly rates can be determined. As shown before (Ertych et al. 2014), all investigated CIN cell lines exhibited increased microtubule plus-end assembly rates during mitosis when compared to chromosomally stable (MIN/MSI) cell lines HCT116 and SW48 (Fig 3.1c). Microtubule dynamics are known to be required for cell migration (Vasiliev et al. 1970). Therefore, interphase microtubule-plus-end assembly rates were measured. Interestingly, only the migratory and invasive CIN cell lines Colo201 and LoVo displayed significantly increased microtubule plus-end assembly rates during interphase compared to the other cell lines (Fig 3.1d). 
a

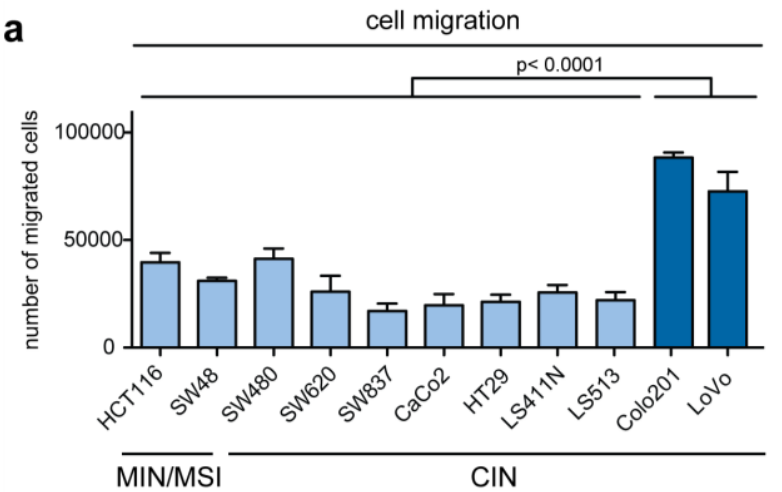

C

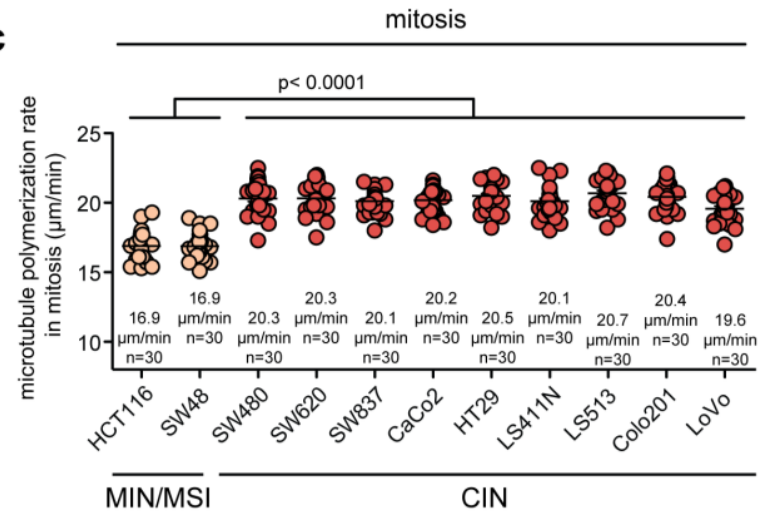

b

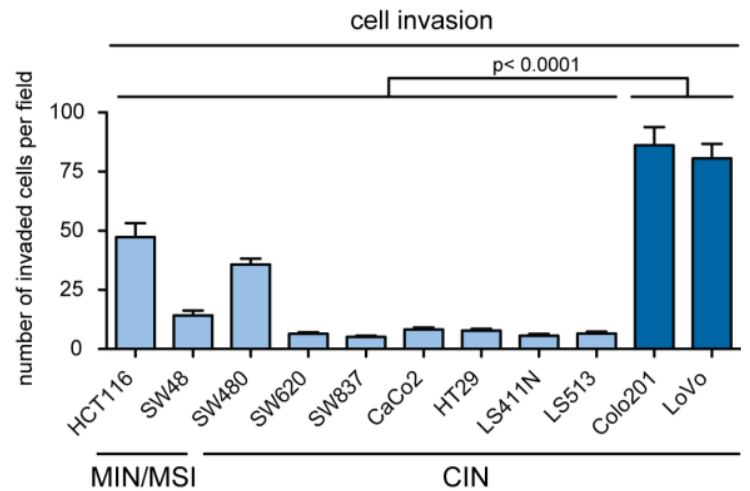

d

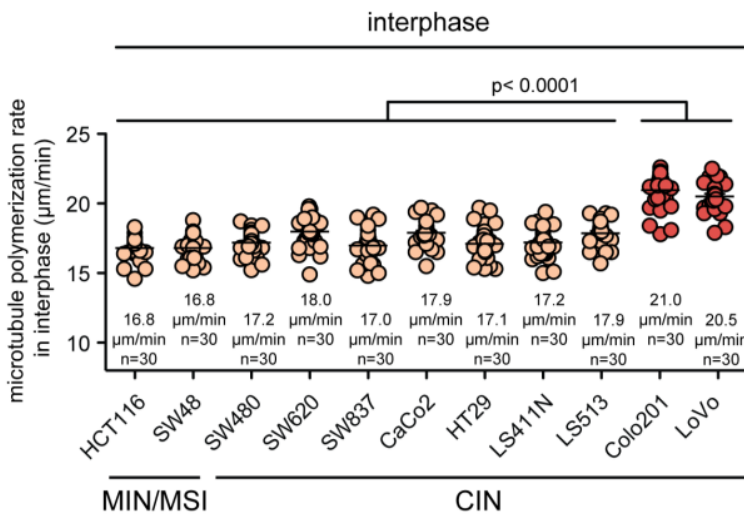

Figure 3.1: Increased interphase microtubule plus-end assembly rates correlate with a migratory and invasive phenotype. (a) Transwell migration assays of a panel of MIN/MSI and CIN CRC cell lines. 200,000 cells were seeded and bar graphs show the proportion of migrated cells after $24 \mathrm{~h}$ (mean \pm sem, $t$-test, $\mathrm{n}=3$ ). (b) Transwell invasion assays for a panel of MIN/MSI and CIN cell lines. 200,000 cells were seeded into matrigel coated cell culture inserts and bar graphs show the number of invaded cells in each picture of 30 randomly chosen pictures taken at $40 x$ magnification from three independent experiments ( $t$-test, mean \pm sem, $n=3$ ). (c) Measurements of mitotic microtubule plus-end assembly rates in a panel of MIN/MSI and CIN CRC cell lines. Cells were treated with $2 \mu \mathrm{M}$ DME for $1 \mathrm{~h}$ prior to determination of microtubule plus-end assembly rates. Scatter dot plots show the average microtubule plus-end assembly rates of 20 microtubules per cell (mean \pm sem, $t$-test, $\mathrm{n}=30$ cells from three independent experiments). (d) Measurements of interphase microtubule plus-end assembly rates using a panel of MIN/MSI and CIN CRC cell lines. Scatter dot plots show the average microtubule plus-end assembly rates of 20 microtubules per cell (mean \pm sem, $t$-test, $n=30$ cells from three independent experiments).

These data indicate that only a subset of CIN cells acquired an abnormal increase in microtubule plus-end assembly rates in interphase, which correlates with increased cell migration and invasion.

To verify our findings, we used different melanoma cell lines that are well characterized regarding their migration and invasion potential. The melanoma cell lines SK-Mel-19 and SKMel-28 are known to be non-invasive, whereas the cell lines SK-Mel-103 and SK-Mel-147 are described to exhibit a highly invasive phenotype (Alla et al. 2010). The migration and invasion potential of these cell lines was validated by transwell migration and invasion assays. Transwell migration assays revealed 6,000 migrated SK-Mel-19 cells and 24,500 
migrated SK-Mel-28, whereas for SK-Mel-103 and SK-Mel-147 migrated cells in a range from 97,000 to 100,500 were detected (Fig. 3.2a). Using transwell invasion assays, 53 and 80 invaded cells per field were observed for SK-Mel-19 and SK-Mel-28, while the highly invasive cell lines SK-Mel-103 and SK-Mel-147 exhibited 366 and 323 invaded cells per field (Fig 3.2b). These four melanoma cell lines were further analyzed with respect to their microtubule plus-end assembly rates during mitosis and interphase by EB3 tracking experiments. Interestingly, the highly migratory and invasive cell lines SK-Mel-103 and SK-Mel-147 exhibited increased microtubule plus-end assembly rates during interphase when compared to the non-invasive cell lines SK-Mel-19 and SK-Mel-28 (Fig 3.2c). Microtubule plus-end assembly rates in interphase were increased from $17.9 \mu \mathrm{m} / \mathrm{min}$ and $18.3 \mu \mathrm{m} / \mathrm{min}$ in SK-Mel19 and SK-Mel-28, respectively, to $21.2 \mu \mathrm{m} / \mathrm{min}$ in both SK-Mel-103 and SK-Mel-147 cells. Apart from that, SK-Mel-19, SK-Mel-28, SK-Mel-103 and SK-Mel-147 displayed increased microtubule plus-end assembly rates during mitosis of $18.7 \mu \mathrm{m} / \mathrm{min}, 19.7 \mu \mathrm{m} / \mathrm{min}, 19.4$ $\mu \mathrm{m} / \mathrm{min}$ and $19.6 \mu \mathrm{m} / \mathrm{min}$ (Fig. 3.2c), when compared to chromosomal stable CRC cell lines HCT116 and SW48 (Fig. 3.1a).
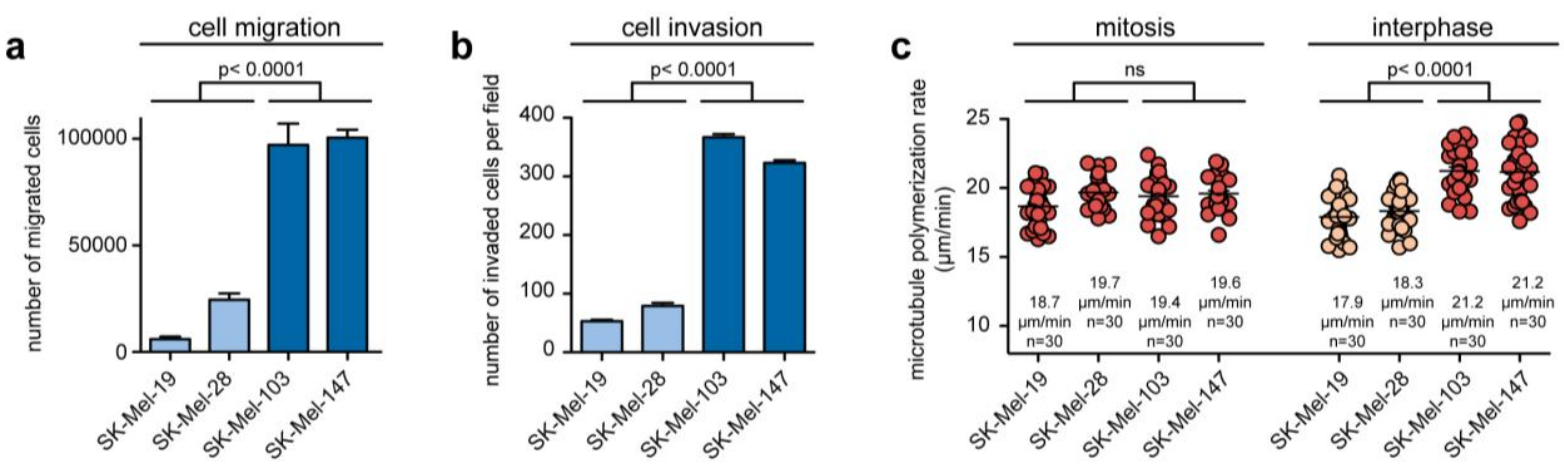

Figure 3.2: Highly migratory and invasive melanoma cell lines SK-Mel-103 and SK-Mel-147 exhibit increased interphase microtubule plus-end assembly rates. (a) Transwell migration assays using a panel of melanoma cell lines. Bar graphs show the proportion of migrated cells from 200,000 seeded cells after $24 \mathrm{~h}$ (mean \pm sem, $t$-test, $\mathrm{n}=3$ ). (b) Transwell invasion assays using a panel of four melanoma cell lines. 200,000 cells were seeded and bar graphs show the number of invaded cells in each picture of 30 randomly chosen pictures taken at $40 x$ magnification from three independent experiments (mean \pm sem, $t$-test, $n=3$ ). (c) Measurements of mitotic and interphase microtubule plus-end assembly rates in a panel of melanoma cell lines. Cells were treated with $2 \mu \mathrm{M}$ DME $2 \mathrm{~h}$ prior to EB3 tracking experiments. Scatter dot plots show the average microtubule plus-end assembly rates of 20 microtubules per cell (mean \pm sem, $t$-test, $n=30$ cells from three independent experiments).

In summary, a subset of the analyzed CRC and melanoma cell lines display increased microtubule plus-end assembly rates in interphase. Additionally, migration and invasion seem to be associated with CIN, but CIN is not per se associated with a high migration and invasion potential. 


\subsubsection{Alterations In Microtubule Plus-End Assembly Rates, But Not A CIN Phenotype Affect Migration And Invasion}

To examine a potential interplay between increased microtubule plus-end assembly rates during interphase and increased cell migration and invasion, enhanced microtubule plus-end assembly rates were restored to normal levels by treatment with the microtubule-binding drug Taxol. Taxol inhibits dynamic microtubule properties and hence stabilizes microtubules (Jordan and Wilson 2004). $2 \mathrm{~h}$ prior to EB3 tracking experiments, cells were treated with 0.2 - $0.5 \mathrm{nM}$ Taxol, which was previously shown to be sufficient to restore proper microtubule plus-end assembly rates in CIN cells (Ertych et al. 2014).

Indeed, at these sub-nanomolar concentrations, Taxol significantly suppressed increased microtubule plus-end assembly rates during mitosis as well as during interphase in both Colo201 and LoVo cells (Fig 3.3a). During mitosis, the microtubule plus-end assembly rates in Colo201 cells were reduced from $20.4 \mu \mathrm{m} / \mathrm{min}$ to $16.9 \mu \mathrm{m} / \mathrm{min}$ and in LoVo cells from 20.2 $\mu \mathrm{m} / \mathrm{min}$ to $17.9 \mu \mathrm{m} / \mathrm{min}$. During interphase, the microtubule plus-end assembly rates in Colo201 cells were decreased from $21.0 \mu \mathrm{m} / \mathrm{min}$ to $16.6 \mu \mathrm{m} / \mathrm{min}$ and in LoVo cells from 20.3 $\mu \mathrm{m} / \mathrm{min}$ to $17.7 \mu \mathrm{m} / \mathrm{min}$. These measured values are comparable to microtubule plus-end assembly rates typically seen in chromosomally stable and non-migratory HCT116 cells (Fig. 3.3a).

a b

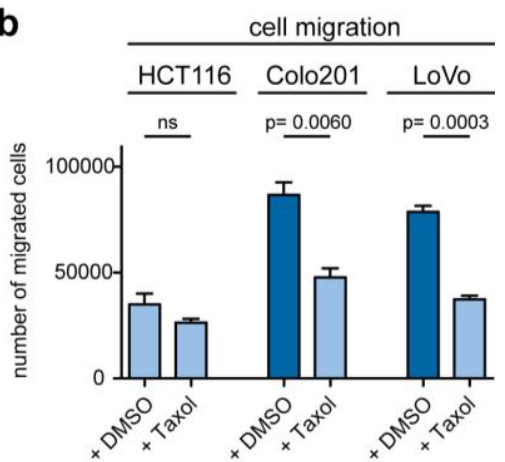

Figure 3.3: Restoration of microtubule plus-end assembly rates suppresses cell migration in CRC cell lines. (a) Measurements of mitotic and interphase microtubule plus-end assembly rates in $\mathrm{CRC}$ cell lines. Cells were treated with DMSO or $0.2 \mathrm{nM}$ Taxol for $2 \mathrm{~h}$. 1-2 $\mathrm{h}$ prior to EB3 tracking experiment, cells were treated with $2 \mu \mathrm{M}$ DME. Scatter dot plots show the average microtubule plusend assembly rates of 20 microtubules per cell (mean \pm sem, $t$-test, $\mathrm{n}=30$ cells from three independent experiments). (b) Transwell migration assays of CRC cell lines. Cells were treated with DMSO or $0.2 \mathrm{nM}$ Taxol. Bar graphs show the proportion of migrated cells from 200,000 seeded cells after $24 \mathrm{~h}$ (mean \pm sem, $t$-test, $\mathrm{n}=3$ ).

Furthermore, transwell migration assays were performed with Taxol treated cells. The migration potential of Colo201 and LoVo cells was significantly decreased upon Taxol treatment in comparison to control DMSO treated cells (Fig 3.3b). In Colo201 cells, the 
number of migrated cells was reduced by approximately $45 \%$ from 86,667 cells upon DMSO treatment to 47,667 cells upon Taxol treatment, whereas for LoVo cells the amount of migrated cells was decreased from 78,667 cells to 37,333 cells on average. In HCT116 cells, Taxol treatment did not have a significant influence on the migration potential. These results indicate an important role of microtubule plus-end assembly rates for cell migration in CRC cell lines. To further substantiate this finding, the melanoma cell lines SK-Mel-19, SK-Mel-28, SK-Mel-103 and SK Mel-147 cells were evaluated regarding their response to Taxol. EB3 tracking experiments were performed with these cell lines. $2 \mathrm{~h}$ prior to determination of microtubule plus-end assembly rates, cells were treated with $0.5 \mathrm{nM}$ Taxol. EB3 tracking experiments revealed a significant decrease in microtubule plus-end assembly rates during both, mitosis and interphase, upon low dose Taxol treatment in all investigated cell lines (Fig 3.4a). Taxol treatment reduced the microtubule plus-end assembly rates in SK-Mel-103 and SK-Mel-147 cells to values comparable to microtubule plus-end assembly rates measured in SK-Mel-19 and SK-Mel-28 cells.

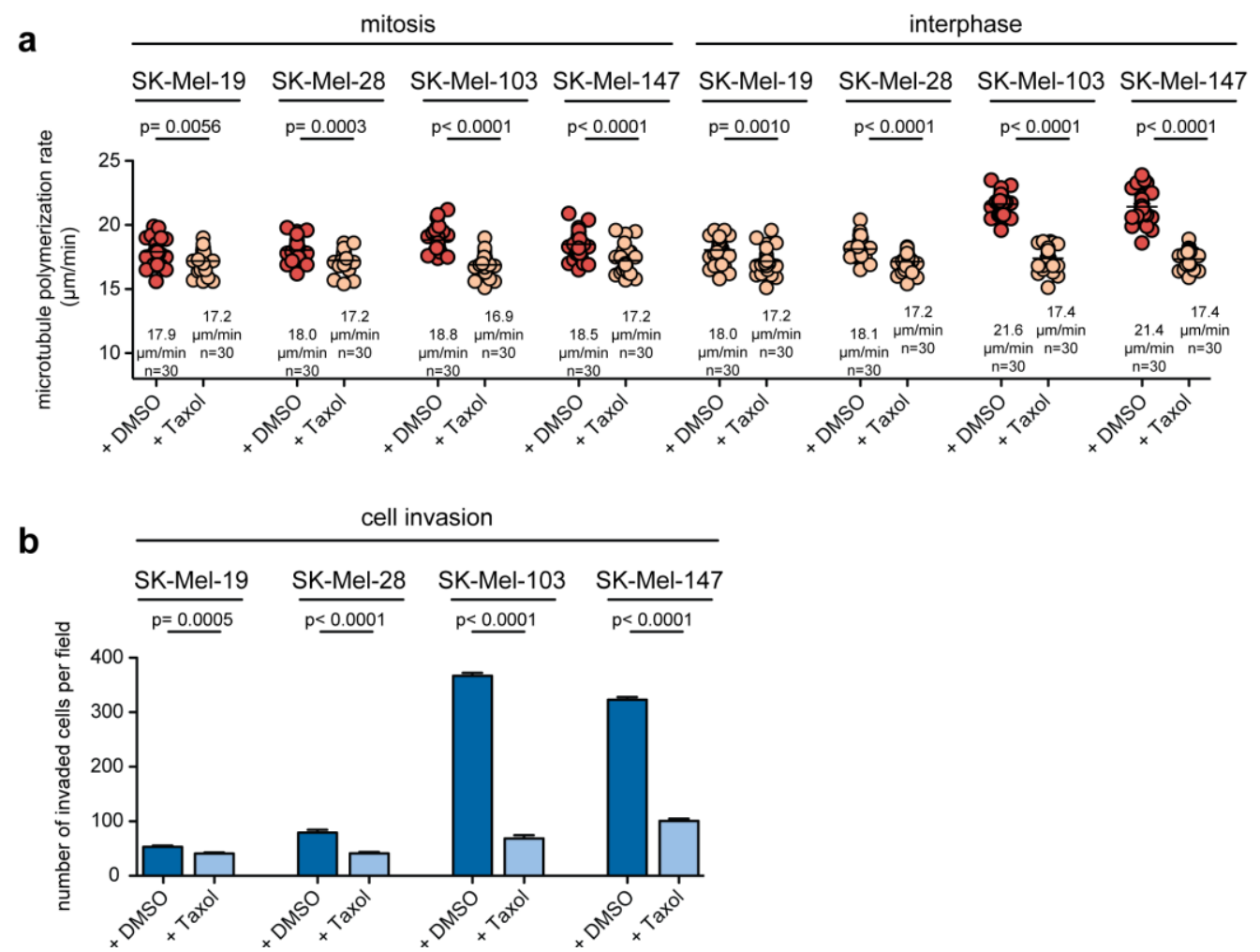

Figure 3.4: Restoration of microtubule plus-end assembly rates suppresses the invasive phenotype in melanoma cells. (a) Measurements of mitotic and interphase microtubule plus-end assembly rates in melanoma cell lines. The indicated cells were treated with DMSO or $0.5 \mathrm{nM}$ Taxol for $2 \mathrm{~h}$. $2 \mathrm{~h}$ prior to EB3 tracking experiments, cells were treated with $2 \mu \mathrm{M}$ DME. Scatter dot plots show the average microtubule plus-end assembly rates of 20 microtubules per cell (mean $\pm \mathrm{sem}, t$ test, $n=30$ cells from three independent experiments). (b) Transwell invasion assays of the indicated melanoma cell lines. Cells were treated with DMSO or $0.5 \mathrm{nM}$ Taxol. 200,000 cells were seeded and the amount of invaded cells after $48 \mathrm{~h}$ in each picture of 30 randomly chosen pictures taken with $40 \mathrm{x}$ magnification from three independent experiments was determined and illustrated as bar graphs (mean \pm sem, $t$-test, $\mathrm{n}=3$ ). 
To further investigate the impact of Taxol on the invasion potential of the melanoma cells, transwell invasion assays were performed. Here, low-dose Taxol treatment significantly decreased the invasive phenotype of SK-Mel-103 and SK-Mel-147 cells. DMSO treated SKMel-103 and SK-Mel-147cells exhibited 366 and 323 invaded cells per field, respectively. Taxol treatment reduced the invaded cells per field to 69 cells in SK-Mel-103 and 100 cells in SK-Mel-147 (Fig 3.4b).

Increased microtubule plus-end assembly rates cause transient spindle geometry defects, which lead to merotelic microtubule-kinetochore attachments (Ertych et al. 2014). Merotelic attachments are erroneous microtubule-kinetochore attachments, which occur, when one kinetochore is attached to microtubules emanating from the two opposing spindle poles. Since the spindle assembly checkpoint does not detect these malattachments, cells can progress into anaphase and display so called lagging chromosomes (Cimini et al. 2001).

To investigate whether increases in microtubule plus-end assembly rates results in the generation of lagging chromosomes in CRC and melanoma cells, cells were synchronized at $G_{1} / S$ transition by double thymidine block and released into the cell cycle and thereby treated with either DMSO or 0.2 - $0.5 \mathrm{nM}$ Taxol for $8.5 \mathrm{~h}$ (CRC cell lines) - $9.5 \mathrm{~h}$ (melanoma cell lines). Cells were fixed and stained for immunofluorescence microscopy and the occurrence of lagging chromosomes during anaphase was analyzed.
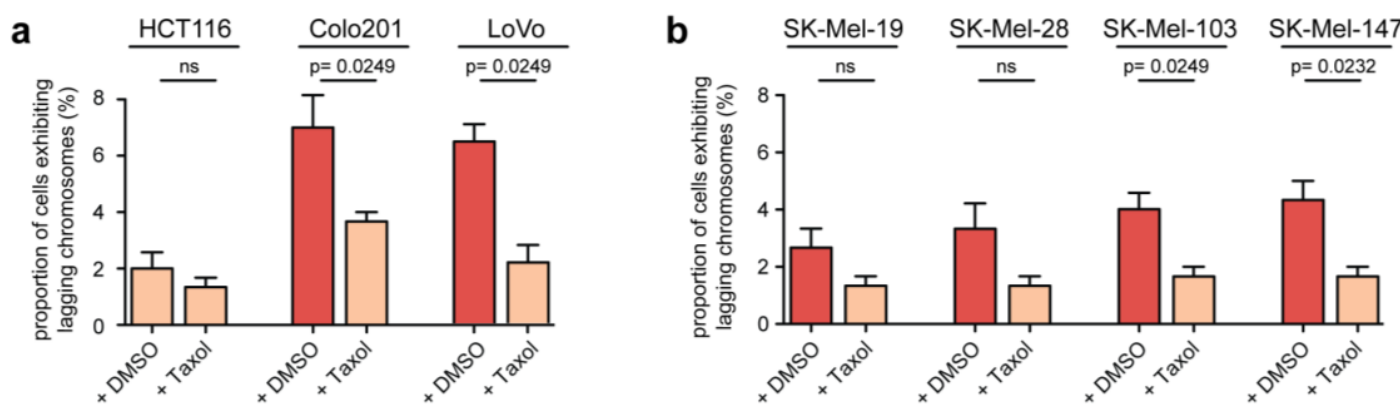

Figure 3.5: Restoration of microtubule plus-end assembly rates by low dose Taxol treatment suppresses the generation of lagging chromosomes. (a) Quantification of cells showing lagging chromosomes during anaphase. CRC cells were synchronized at $\mathrm{G}_{1} / \mathrm{S}$ transition by a double thymidine block and released into the cell cycle thereby treating with DMSO or $0.2 \mathrm{nM}$ Taxol. After $8.5 \mathrm{~h}$ cells were fixed and stained for immunofluorescence microscopy and the occurrence of lagging chromosomes was analyzed. Bar graphs show proportion of cells displaying at least one CRESTpositive chromatid (mean \pm sem, $t$-test, $n=3$ with a total of 300 anaphase cells). (b) Quantification of cells with lagging chromosomes during anaphase using melanoma cell lines. Cells were synchronized at $\mathrm{G}_{1} / \mathrm{S}$ transition by a double thymidine block and released into the cell cycle thereby treating with DMSO or $0.5 \mathrm{nM}$ Taxol. After $9.5 \mathrm{~h}$ cells were fixed and stained for immunofluorescence microscopy and the occurrence of lagging chromosomes was analyzed. Bar graphs show quantification of cells displaying at least one CREST-positive chromatid (mean \pm sem, $t$-test, $n=3$ with a total of 300 anaphase cells).

The proportion of cells exhibiting lagging chromosomes in Colo201 and LoVo cells was significantly decreased after low dose Taxol treatment in comparison to DMSO treated cells. 
On average $6 \%$ of DMSO treated Colo201 cells exhibited lagging chromosomes. This proportion was reduced to $3.6 \%$ upon Taxol treatment. Also in LoVo cells, the occurrence of lagging chromosomes was reduced from $6 \%$ to $2 \%$. However, Taxol treatment had no significant influence onto the generation of lagging chromosomes in HCT116 cells (Fig 3.5a). The melanoma cell lines investigated here have not been characterized regarding the generation of lagging chromosomes. But the increased microtubule plus-end assembly rates during mitosis (Fig 3.2c) hint to a chromosomally instable phenotype. DMSO treated melanoma cells exhibited lagging chromosomes in a range from $3 \%$ to $5 \%$. This proportion was reduced to $1 \%$ to $2 \%$ upon low dose Taxol treatment. Thus, also in melanoma cell lines, increased microtubule plus-end assembly rates are associated with the generation of lagging chromosomes, albeit at lower frequency when compared to the CRC cell lines.

Hence, Taxol treatment decreased microtubule plus-end assembly rates in mitotic and interphase cells and reduced both the occurrence of lagging chromosomes and the migratory and invasive phenotype in CRC and melanoma cell lines. Since the generation of lagging chromosomes is tightly associated with $\mathrm{CIN}$, the question arose, whether a CIN phenotype per se might be responsible for the increased migration and invasion potential.

The occurrence of lagging chromosomes can be specifically suppressed in the presence of increased microtubule plus-end assembly rates by overexpression of the mitotic centromereassociated kinesin MCAK (Ertych et al. 2014). MCAK is a microtubule depolymerase, which destabilizes kinetochore microtubules at the metaphase to anaphase transition, thus resolving erroneous kinetochore-microtubule-attachments (Maney et al. 1998; Bakhoum et al. 2009).

MCAK was overexpressed in the CRC cell lines HCT116 and Colo201, as well as in the melanoma cell line SK-Mel-103. The overexpression of MCAK was verified by western blot analyses (Fig 3.6a). Cells were synchronized at $G_{1} / S$ transition, released into the cell cycle for $8.5 \mathrm{~h}$ (CRC cell lines) - $9.5 \mathrm{~h}$ (melanoma cell line) and fixed and stained for immunofluorescence microscopy. MCAK overexpression decreased the number of cells with lagging chromosomes in all cell lines in comparison to control vector transfected cells (Fig 3.6b). In Colo201 cells, the amount of lagging chromosomes was reduced from $6.3 \%$ to $1.6 \%$, whereas in SK-Mel-103 cells the amount was reduced from $4 \%$ to $1.6 \%$ on average. These reductions in the occurrence of lagging chromosomes are comparable to those obtained by Taxol treatment (Fig 3.5a-b), but EB3 tracking experiments revealed no significantly altered microtubule plus-end assembly rates upon MCAK overexpression (Fig 3.6c). 
a

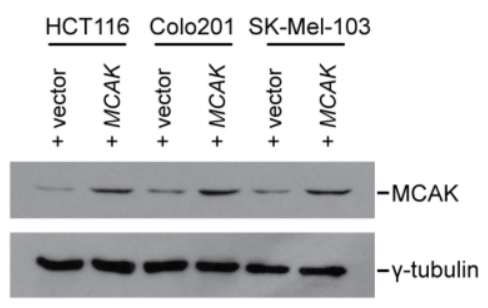

b $\frac{\text { HCT116 }}{\mathrm{ns}} \stackrel{\text { Colo201 }}{\mathrm{p=0.0078}} \frac{\text { SK-Mel-103 }}{\mathrm{p}=0.0147}$

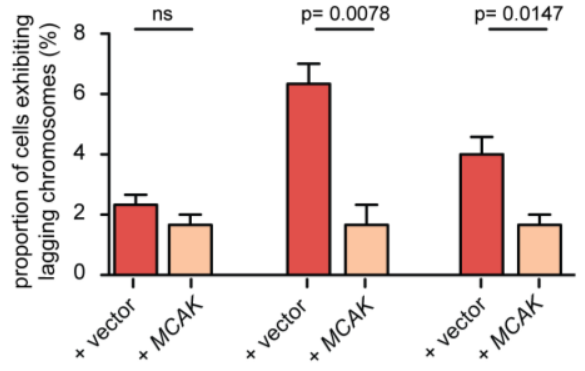

c

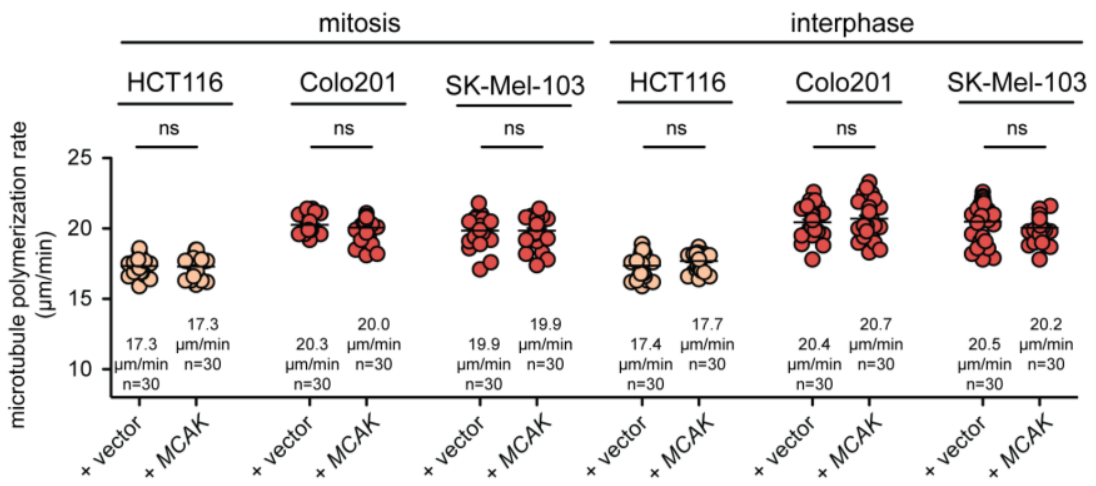

d

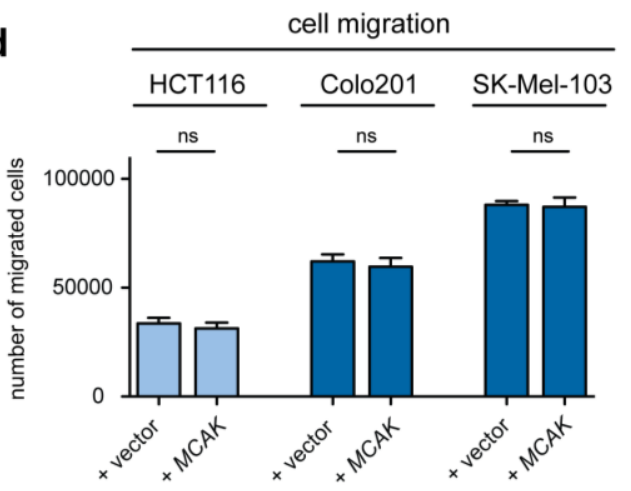

e

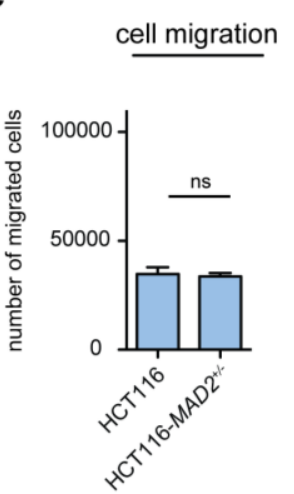

Figure 3.6: CIN does not trigger cell migration per se. (a) Representative western blot of HCT116, Colo201 and SK-Mel-103 cells detecting MCAK after overexpression of MCAK. y-tubulin served as loading control. (b) The occurrence of anaphase cells with lagging chromosomes was analyzed in HCT116, Colo201 and SK-Mel-103 cells after overexpression of MCAK. Cells were synchronized at $\mathrm{G}_{1} / \mathrm{S}$ transition by double thymidine block, released into the cell cycle for $8.5 \mathrm{~h}$ (HCT116, Colo201) or $9.5 \mathrm{~h}$ (SK-Mel-103) and fixed and stained for immunofluorescence microscopy. Bar graphs show proportion of cells exhibiting at least one CREST-positive chromatid (mean $\pm \mathrm{sem}, t$-test, $\mathrm{n}=3$ with a total of 300 anaphase cells). (c) Measurements of microtubule plus-end assembly rates during mitosis and interphase after overexpression of MCAK. Scatter dot plots show the average microtubule plus-end assembly rates of 20 microtubules per cell (mean $\pm \mathrm{sem}, t$-test, $\mathrm{n}=30$ cells from three independent experiments). (d) Transwell migration assays of HCT116, Colo201 and SK-Mel-103 cells were performed with or without overexpression of MCAK. 200,000 cells were seeded and bar graphs show the amount of migrated cells after $24 \mathrm{~h}$ (mean \pm sem, $t$-test, $\mathrm{n}=3$ ). (e) Transwell migration assay of HCT116 and HCT116 MAD2 $2^{+/} .200,000$ cells were seeded and bar graphs show the amount of migrated cells after $24 \mathrm{~h}$ (mean \pm sem, $t$-test, $\mathrm{n}=3$ ).

To investigate a potential link between CIN and migration, transwell migration assays were performed. These experiments revealed no influence of MCAK overexpression on the 
migration potential of Colo201 and SK-Mel-103 cells (Fig 3.6d). Additionally HCT116-MAD2 ${ }^{+-}$ cells were analyzed regarding their migration potential. These cells have a mitotic checkpoint defect and frequently missegregate chromosomes. But the HCT116-MAD2 ${ }^{+/}$cells did not show increased cell migration compared to parental HCT116 cells (Fig. 3.6e). Thus the occurrence of lagging chromosomes per se did not influence the migration potential of the highly migratory cell lines Colo201 and SK-Mel-103. Instead, abnormally increased microtubule plus-end assembly rates in mitosis and interphase are associated with the generation of lagging chromosomes and increased cell migration, respectively.

Microtubule plus-end assembly is mediated by the microtubule polymerase ch-TOG (encoded by the CKAP5 gene) (Gard \& Kirschner 1987; Brouhard et al. 2008). In order to further investigate the influence of increased microtubule plus-end assembly rates during migration and invasion and also to exclude unspecific effects of the Taxol treatment, CKAP5 was repressed by siRNA in SK-Mel-103 and Colo201 cells. HCT116 was used as a control cell line. The repression of CKAP5 was verified by western blot analyses (Fig 3.7a). Live cell microscopy experiments revealed a significant decrease of microtubule plus-end assembly rates in SK-Mel-103 and Colo201 cells to normal levels during both, mitosis and interphase, upon siRNA-mediated repression of CKAP5 in comparison to LUCIFERASE siRNA transfected cells (Fig 3.7b). In HCT116 cells, microtubule plus-end assembly rates were not significantly influenced upon CKAP5 repression (Fig. 3.7b).

Furthermore, repression of CKAP5 also led to a significant decrease in the amount of cells exhibiting lagging chromosomes in Colo201 and SK-Mel-103 cells compared to LUCIFERASE siRNA transfected cells. In Colo201 cells, the number of cells showing lagging chromosomes was reduced from $6.7 \%$ to $3.6 \%$, whereas in SK-Mel-103 cells the amount was reduced by $50 \%$ from $4 \%$ to $2 \%$. (Fig $3.7 \mathrm{c}$ ).

Following that, transwell migration and invasion assays were performed. Here, SK-Mel-103 and Colo201 cells displayed a significantly decreased migration and invasion potential after CKAP5 repression in comparison to control transfected cells (Fig 3.7d-e). Upon LUCIFERASE siRNA transfection, Colo201 and SK-Mel-103 exhibited on average 78,333 migrated cells and 94,250 migrated cells, respectively, which were reduced to 53,417 cells and 72,250 cells upon CKAP5 repression. Transwell invasion assays revealed on average 92 invaded Colo201 cells, which were reduced to 37 invaded cells per field upon CKAP5 repression, and 353 invaded SK-Mel-103 cells, which were decreased to 254 cells per field after CKAP5 repression.

The data upon repression of CKAP5 substantiates the findings obtained upon low dose Taxol treatment: $A$ decrease in microtubule plus-end assembly rates also decreased the migratory and invasive phenotype. 
a
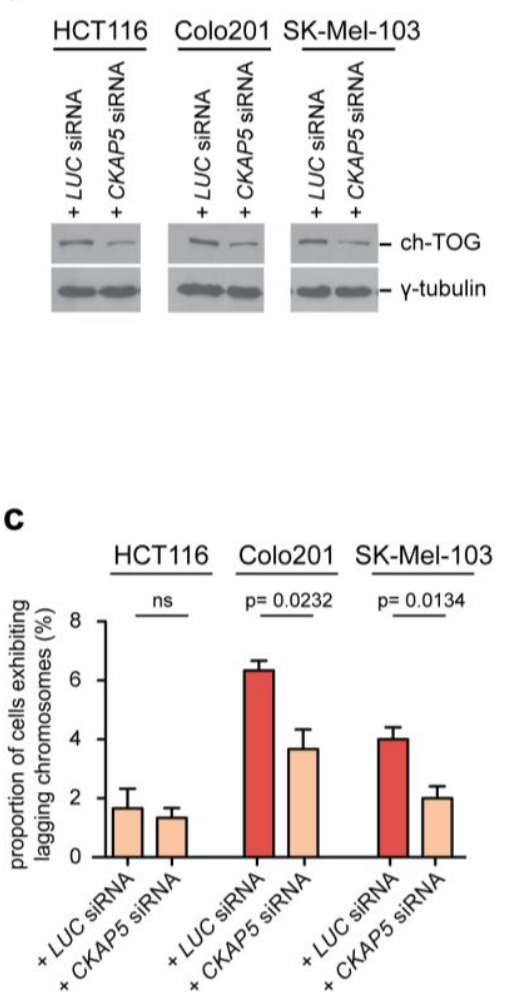

b

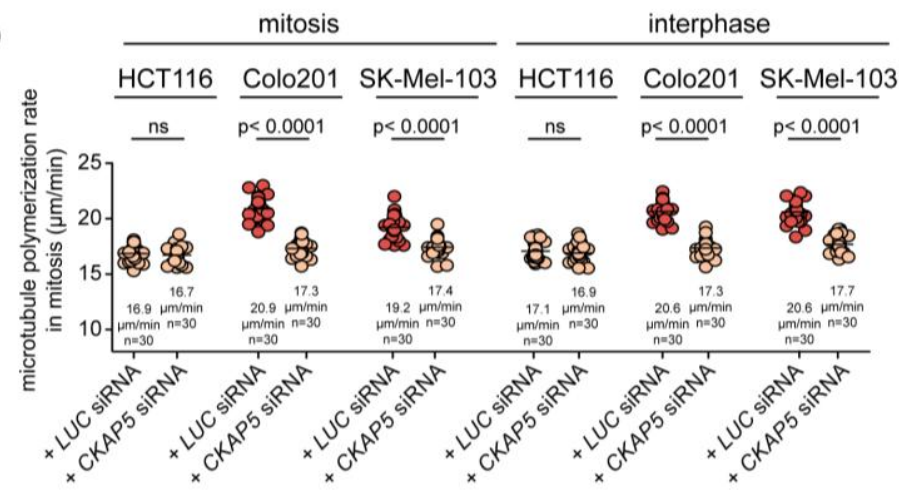

d

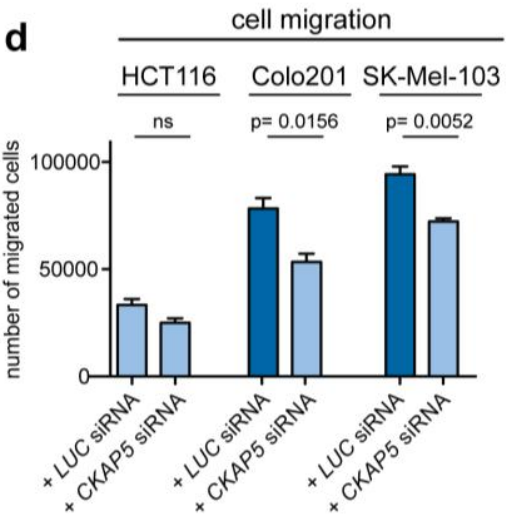

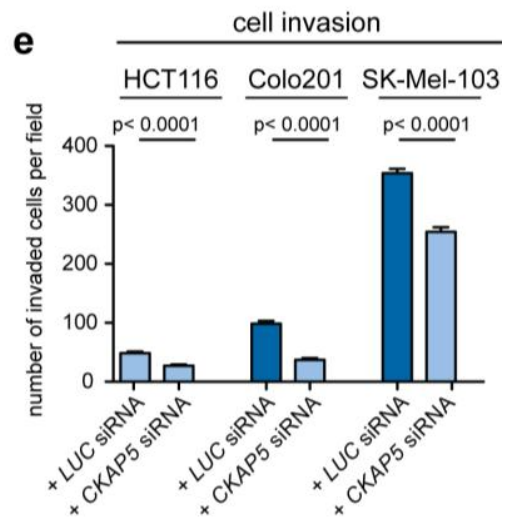

Figure 3.7: Inhibition of increased microtubule plus-end assembly rates suppresses migration and invasion. (a) Representative western blot detecting ch-TOG using HCT116, Colo201 and SK-Mel-103 cells transfected with 60 pmol LUCIFERASE or CKAP5 siRNA. y-tubulin served as loading control. (b) Measurements of mitotic and interphase microtubule plus-end assembly rates after repression of CKAP5. HCT116, Colo201 and SK-Mel-103 cells were transfected with 60 pmol LUCIFERASE or CKAP5 siRNA. $1 \mathrm{~h} \mathrm{-} 2 \mathrm{~h}$ prior to EB3 tracking experiments, cells were treated with $2 \mu \mathrm{M}$ DME. Scatter dot plots show the average microtubule plus-end assembly rates of 20 microtubules per cell (mean \pm sem, $t$-test, $\mathrm{n}=30$ cells from three independent experiments). (c) Quantification of cells exhibiting lagging chromosomes after CKAP5 or LUCIFERASE siRNA transfection as described in (a). Cells were synchronized at $\mathrm{G}_{1} / \mathrm{S}$ transition by double thymidine block, released into the cell cycle for $8.5-9.5 \mathrm{~h}$ and fixed and stained for immunofluorescence microscopy. Cells displaying at least one CREST-positive chromatid were counted as positive. Bar graphs show proportion of cells with lagging chromosomes (mean \pm sem, $t$-test, $\mathrm{n}=3$ with a total of 300 anaphase cells). (d) Transwell migration assays after CKAP5 or LUCIFERASE siRNA transfection as described in (a). 200,000 cells were seeded into cell culture inserts and bar graphs show the number of migrated cells after $24 \mathrm{~h}$. (mean \pm sem, $t$-test, $\mathrm{n}=3$ ) (e) Transwell invasion assays after CKAP5 or LUCIFERASE siRNA transfection as described in (a). 200,000 cells were seeded into cell culture inserts. After $48 \mathrm{~h}$, the number of invaded cells in each picture of 30 randomly chosen pictures was determined. Pictures were taken with 40x magnification. Bar graphs show number of invaded cells per picture (mean $\pm \mathrm{sem}, t$-test, $n=3$ ).

Furthermore, it was of interest to investigate, whether an increase in microtubule plus-end assembly rates per se induces a migratory and invasive phenotype. To address this question, the microtubule polymerase ch-TOG/CKAP5 or a control vector were overexpressed or cells were treated with low doses of Nocodazole. Our group showed recently that low concentrations of the microtubule destabilizing drug Nocodazole increased microtubule plus-end assembly rates (Ertych et al. 2014). HCT116 and SK-Mel-28 cells, 
which neither exhibit increased microtubule plus-end assembly rates nor a migratory and invasive phenotype, were used for this approach.

The overexpression of CKAP5 was verified by western blot analyses (Fig. 3.8a). EB3 tracking experiments were performed and revealed a significant increase in microtubule plusend assembly rates during both, interphase and mitosis, upon CKAP5 overexpression in comparison to control vector (Fig 3.8b). During mitosis, microtubule pus-end assembly rates were increased on average from $16.9 \mu \mathrm{m} / \mathrm{min}$ to $20.3 \mu \mathrm{m} / \mathrm{min}$ in HCT116 cells and 18.3 $\mu \mathrm{m} / \mathrm{min}$ to $20.7 \mu \mathrm{m} / \mathrm{min}$ in SK-Mel-28 cells. During interphase, microtubule pus-end assembly rates were increased on average from $17.3 \mu \mathrm{m} / \mathrm{min}$ to $20.3 \mu \mathrm{m} / \mathrm{min}$ in HCT116 cells and from $17.9 \mu \mathrm{m} / \mathrm{min}$ to $20.6 \mu \mathrm{m} / \mathrm{min}$ in SK-Mel-28 cells. Under these conditions, transwell migration and invasion assays were performed to analyze the migratory and invasive phenotype after CKAP5 overexpression (Fig 3.8c-d). Both, the migration and invasion potentials, of HCT116 and SK-Mel-28 cells were significantly increased after CKAP5 overexpression. The migration potential was increased from 37,083 migrated HCT116 cells and 34,000 migrated SK-Mel-28 cells to 69,417 and 71,583 migrated cells, respectively. In HCT116 cells, the transwell invasion assays revealed on average 55 invaded cells per field after control vector transfection and 143 invaded cells upon CKAP5 overexpression. In SK-Mel-28 cells, the number of invaded cells was increased from 66 invaded cells to 169 invaded cells upon CKAP5 overexpression. As hypothesized from the induction of increased microtubule plusend assembly rates during mitosis, HCT116 and SK-Mel-28 cells exhibited increased numbers of cells exhibiting lagging chromosomes after overexpression of CKAP5 (Fig 3.8e). In HCT116 cells the proportion was increased from $1.6 \%$ to $4 \%$ and in SK-Mel-28 cells from $2 \%$ to $4.6 \%$ upon CKAP5 overexpression.

In addition to CKAP5 overexpression, cells were treated with $2 \mathrm{nM}$ Nocodazole $2 \mathrm{~h}$ prior to the determination of microtubule plus-end assembly rates. Indeed, after Nocodazole treatment, cells exhibited increased microtubule plus-end assembly rates that were comparable to microtubule plus-end assembly rates measured upon CKAP5 overexpression (Fig 3.8b). Transwell migration and invasion assays revealed a significantly increased migration and invasion potential (Fig 3.8bc-d), which was also comparable to the effects obtained upon CKAP5 overexpression. Analyses of cells displaying lagging chromosomes revealed a slight increase in their occurrenc. In HCT116 cells, low dose Nocodazole treatment increased the number of lagging chromosomes from $1.5 \%$ to $3.25 \%$ on average, whereas in SK-Mel-28 cells this amount was increased from $1.6 \%$ to $3.3 \%$ in comparison to DMSO treated cells (Fig. 3.8e). 
a

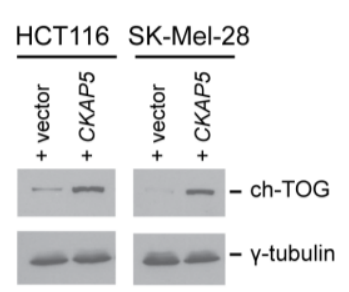

b

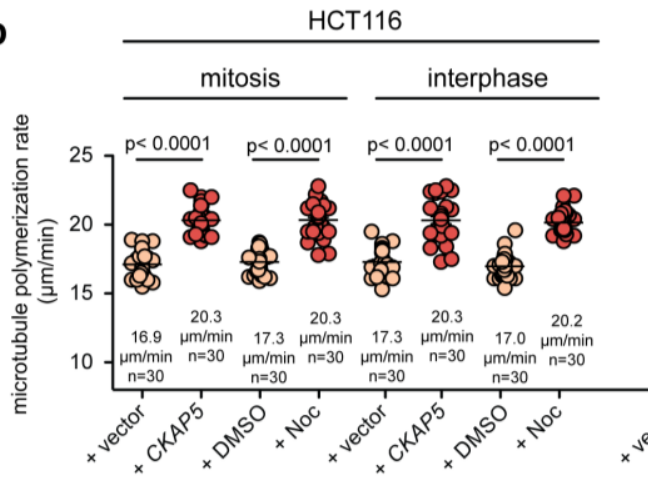

SK-Mel-28

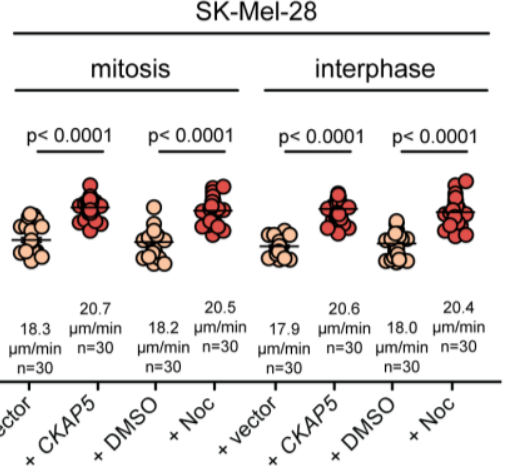

C

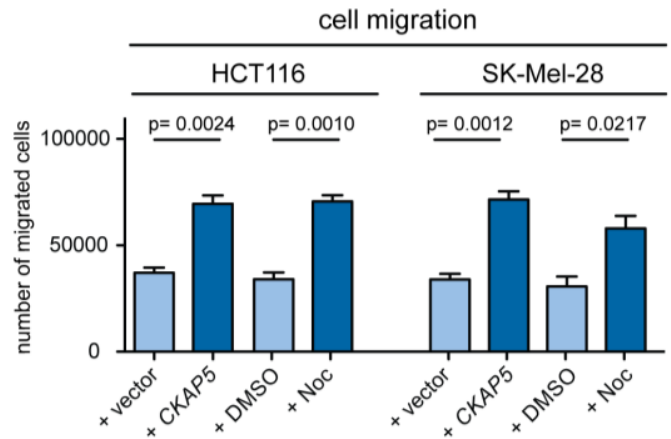

e

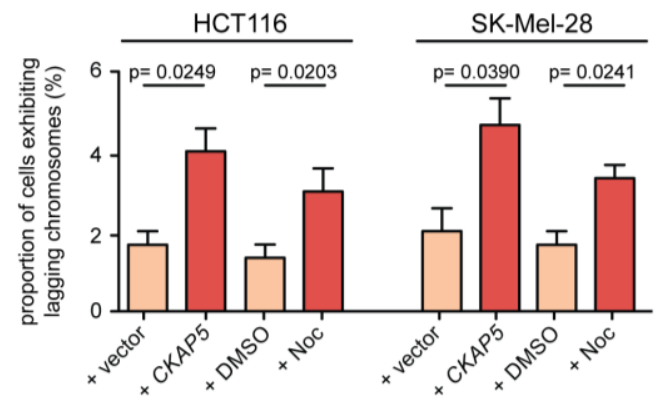

d

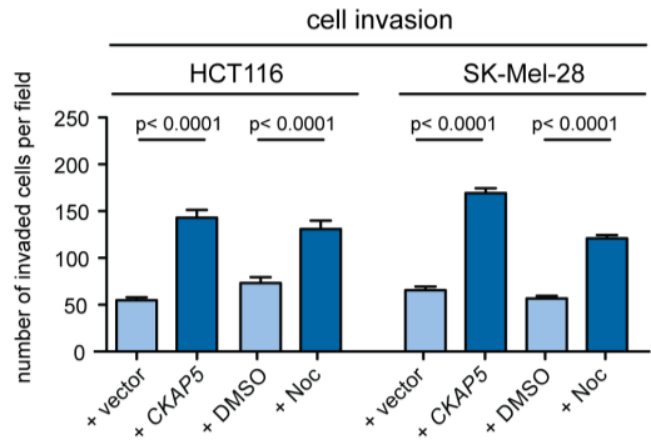

Figure 3.8: Induction of increased microtubule plus-end assembly rates triggers migration and invasion. (a) Representative western blot of HCT116 and SK-Mel-28 cells after overexpression of CKAP5. y-tubulin served as loading control. (b) Measurements of mitotic and interphase microtubule plus-end assembly rates after overexpression of CKAP5 or treatment with $2 \mathrm{nM}$ Nocodazole for $2 \mathrm{~h}$. Cells were treated with $2 \mu \mathrm{M}$ DME $1 \mathrm{~h}-2 \mathrm{~h}$ prior to EB3 tracking experiments. Scatter dot plots show the average microtubule plus-end assembly rates of 20 microtubules per cell (mean \pm sem, $t$-test, $\mathrm{n}=30$ cells from three independent experiments). (c) Transwell migration assays after overexpression of CKAP5 or treatment with $2 \mathrm{nM}$ Nocodazole. 200,000 cells were seeded into cell culture inserts. Bar graphs show number of migrated cells after $24 \mathrm{~h}$ (mean \pm sem, $t$-test, $\mathrm{n}=3$ ) (d) Transwell invasion assays after overexpression of CKAP5 or treatment with $2 \mathrm{nM}$ Nocodazole. 200,000 cells were seeded into cell culture inserts. Bar graphs show the number of invaded cells in every picture of 30 randomly chosen pictures from three independent experiments (mean \pm sem, $t$-test, $n=3$ ). (e) Quantification of cells with lagging chromosomes after over expression of CKAP5 or treatment with $2 \mathrm{nM}$ Nocodazole. Cells were synchronized at $\mathrm{G}_{1}$-S-transition by double thymidine block, released into the cell cycle for $8.5 \mathrm{~h}-9.5 \mathrm{~h}$ and fixed and stained for immunofluorescence microscopy. Cells displaying at least one CREST-positive chromatid were quantified. Bar graphs show the proportion of cells with lagging chromosomes (mean \pm sem, $t$-test, $n=3-4$ with a total of 300-400 anaphase cells). 


\subsubsection{Metastasis Associated Alterations In Human Cancer Induce Increased Microtubule Plus-End Assembly Rates}

In human cancers, various alterations in gene expression are associated with a highly migratory and invasive phenotype. To address the question, whether the correlation between migration and invasion and altered microtubule dynamics can be detected also in cancer associated settings different gene alterations with prognostic significance were tested for their influence on microtubule dynamics. Thereby, inactivation of the retinoblastoma protein $\mathrm{Rb}$ is associated with the development of invasive cancers (Labrecque et al. 2016). In addition, overexpression of $E 1 A$ and $E 7$ leads to inactivation of $R b$. Furthermore, the overexpression of the receptor for hyaluronan-mediated motility (Rhamm/HMMR) is known to promote cellular motility and invasion (Maxwell et al. 2008; Crainie et al. 1999). Additionally, increased TGF- $\beta$ signaling is highly correlated with metastasis in several cancer entities and known to induce EMT and to increase cell migration (Dalal et al. 1993; Meulmeester \& Ten Dijke 2010).

HCT116 and SK-Mel-28 cells were used for siRNA-mediated repression of $R B$, for overexpression of $E 7, E 1 A$ and $H M M R$ or for treatment with TGF- $\beta$. Transwell migration and invasion assays were conducted and confirmed the highly migratory and invasive potential after these treatments (Fig. 3.9a-b). Interestingly, in EB3 tracking experiments, $R B$ repression, E7, E1A and $H M M R$ overexpression or treatment with $10 \mathrm{ng} / \mathrm{ml}$ TGF- $\beta$ for $3 \mathrm{~h}$ significantly increased microtubule plus-end assembly rates in mitosis and interphase in both cell lines (Fig. 3.9c-d). In HCT116 cells, the indicated treatments increased microtubule plusend assembly rates in mitosis from 16.7-16.8 $\mu \mathrm{m} / \mathrm{min}$ in control cells to $19.5-20.2 \mu \mathrm{m} / \mathrm{min}$ and in interphase from a range of 16.7-16.8 $\mu \mathrm{m} / \mathrm{min}$ to $20.2-20.3 \mu \mathrm{m} / \mathrm{min}$. In mitotic SK-Mel-28 cells, elevated microtubule polymerization rates of $19.5-20.5 \mu \mathrm{m} / \mathrm{min}$ were measured compared to $18.4-19.3 \mu \mathrm{m} / \mathrm{min}$ on average in control cells. During interphase, the indicated treatments increased microtubule plus-end assembly rates from $18.3-18.4 \mu \mathrm{m} / \mathrm{min}$ to polymerization rates ranging from $20.3-20.6 \mu \mathrm{m} / \mathrm{min}$.

Thus, cancer relevant alterations, which are described to be associated with a highly invasive phenotype are accompanied by increased mitotic and interphase microtubule plus-end assembly rates. 

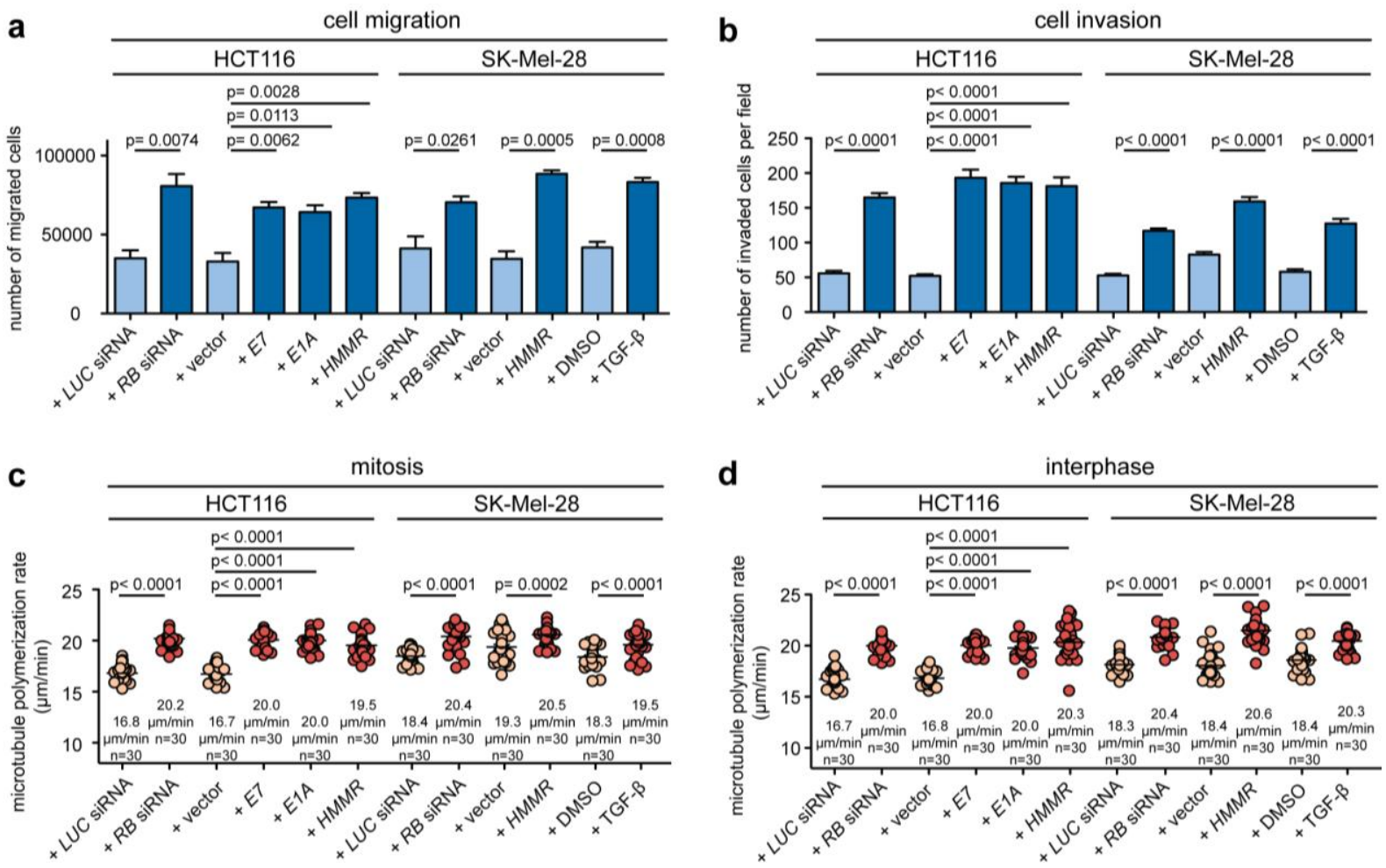

Figure 3.9: Invasion-associated alterations in cancer correlate with increased microtubule plusend assembly rates. (a) Transwell migration assays of HCT116 and SK-Mel-28 cells after transfection with 60 pmol LUCIFERASE or RB siRNA, or overexpression of E7, E1A or HMMR, or after treatment with $10 \mathrm{ng} / \mathrm{ml}$ TGF- $\beta$. 200,000 cells were seeded and bar graphs show the amount of migrated cells after $24 \mathrm{~h}$ (mean \pm sem, $t$-test, $\mathrm{n}=3$ ). (b) Transwell invasion assays of HCT116 and SKMel-28 cells after the indicated treatments. 200,000 cells were seeded. Bar graphs show the number of invaded cells in every picture of 30 randomly chosen pictures from three independent experiments (mean \pm sem, $t$-test, $n=3$ ). (c) EB3 tacking experiments of HCT116 and SK-Mel-28 cells in mitosis after the indicated treatments. Scatter dot plots show the average microtubule plus-end assembly rates of 20 microtubules per cell (mean \pm sem, $t$-test, $n=30$ cells from three independent experiments). (d) EB3 tracking experiment of HCT116 and SK-Mel-28 cells in interphase after the indicated treatments. Scatter dot plots show the average microtubule plus-end assembly rates of 20 microtubules per cell (mean \pm sem, $t$-test, $n=30$ cells from three independent experiments).

\subsubsection{The Microtubule Plus-End Binding Protein EB1 Is Important For Microtubule-Dependent Signaling}

The previous observations indicate a strong correlation between abnormally increased microtubule plus-end assembly rates and a migratory and invasive phenotype. Migration and invasion is mainly driven by different actin filament structures and their remodeling. But also microtubules are described to play a role during cell motility (Waterman-Storer \& Salmon 1999). Still the question arises, how changes in microtubule dynamics affect cellular motility. An interesting hypothesis is that microtubule plus-ends might form moving signaling platforms, in which the end-binding protein EB1 plays an important role. Thus, it was of interest to investigate, whether the repression of EB1 in human cancer cells would affect CIN and cell migration. Therefore, EB1 was partially repressed by the use of siRNA in SW620 
and SK-Mel-103 cells. Repression of EB1 was verified by western blot analyses (Fig. 3.10a). Interestingly, EB1 knock down did not affect microtubule plus-end assembly rates neither in mitosis nor in interphase (Fig 3.10b). However, the occurrence of cells with lagging chromosomes was significantly decreased (Fig 3.10c). Moreover, SK-Mel-103 cells showed a significant decreased migration potential upon EB1 repression in transwell migration assays (Fig. 3.10d).

a

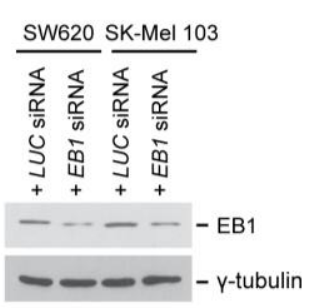

C

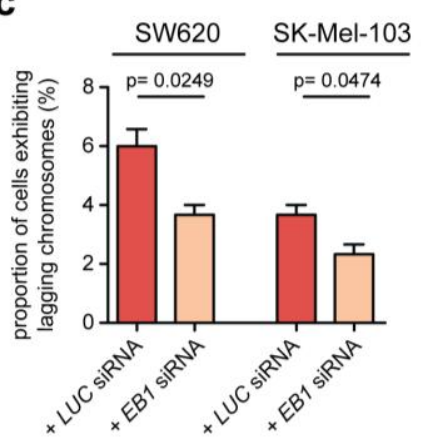

b
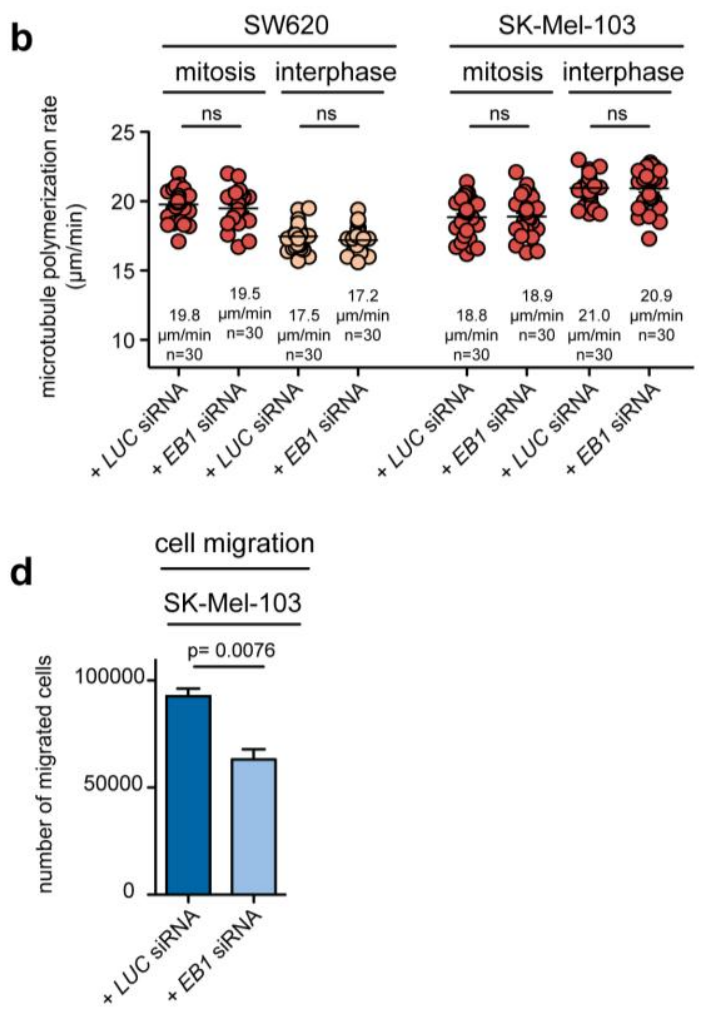

Figure 3.10: Repression of EB1 reduces the migratory and CIN phenotype while having no impact on microtubule plus-end assembly rates. (a) Representative western blot of SW620 and SK-Mel-103 cells after repression of EB1. Cells were transfected with $80 \mathrm{pmol}$ LUCIFERASE or EB1 siRNA. $\mathrm{y}$-tubulin served as loading control (b) Measurements of microtubule plus-end assembly rates in mitosis and interphase after repression of EB1. SW620 and SK-Mel-103 cells were transfected with 80 pmol LUCIFERASE or EB1 siRNA. Scatter dot plots show the average microtubule plus-end assembly rates of 20 microtubules per cell (mean \pm sem, $t$-test, $n=30$ cells from three independent experiments). (c) Quantification of cells exhibiting lagging chromosomes after repression of EB1. Cells were synchronized at $G_{1} / S$ transition by double thymidine block, released into the cell cycle for $8.5 \mathrm{~h}-9.5 \mathrm{~h}$ and fixed and stained for immunofluorescence microscopy. Cells displaying at least one CREST-positive chromatid were counted as positive. Bar graphs show the proportion of cells displaying lagging chromosomes (mean \pm sem, $t$-test, $n=3$ with a total of 300 anaphase cells). (d) Transwell migration assays after repression of EB1. 200,000 cells were seeded and bar graphs show the number of migrated cells after $24 \mathrm{~h}$ (mean \pm sem, $t$-test, $\mathrm{n}=3$ )..

These results revealed that EB1 is involved in triggering enhanced migration and $\mathrm{CIN}$ in SK-Mel-103 and SW620 cells in response to increased microtubule plus-end assembly rates. Since partial EB1 repression did not alter the microtubule plus-end assembly rates itself, the results suggest, that EB1 acts downstream of microtubules. 


\subsubsection{SW620 And SK-Mel-103 Cells Exhibit Elevated Levels Of Active TRIO}

The repression of $E B 1$ seems to disrupt a signaling pathway, which is important for migration and invasion downstream of microtubule dynamics. Therefore, it was of utmost interest to investigate, how EB1-derived signaling affect cellular motility. Recently, the binding of EB1 to TRIO was described (Van Haren et al. 2014). TRIO is a guanine nucleotide exchange factor (GEF) for Rac1 and is part of a gene signature of genes implicated in cell migration (referred to as HCCS24) as well as of the HET70 signature of genes associated with aneuploidy (Kohn et al. 2012, Sheltzer 2013;). Therefore, TRIO represents an interesting candidate that may link microtubule dynamics to $\mathrm{CIN}$ and cell migration.

To test for an involvement of TRIO in CIN and migration, GEF assays were performed to analyze TRIO activity in chromosomally instable SW620 and highly migratory SK-Mel-103 cells. For this, an agarose bead coupled nucleotide-free Rac1 mutant (Rac1 G15A) that binds to activated GEFs with increased affinity was used to pull down active TRIO.

TRIO activity in chromosomal instable SW620 cells was compared to chromosomal stable HCT116 cells during mitosis. Cells were arrested in mitosis by treatment with $2 \mu \mathrm{M} D M E$ for $16 \mathrm{~h}$. Additionally, cells were treated with DMSO or $0.2 \mathrm{nM}$ Taxol. Treatment with the TRIO specific inhibitor ITX3 (Bouquier et al. 2009) served as a control. Western blot analyses revealed similar levels of endogenous TRIO in HCT116 and SW620 cells, which were not altered upon Taxol or ITX3 treatment (depicted as input) (Fig 3.11a). However, an increased proportion of active TRIO was immunoprecipitated using the GST-Rac1 G15A agarose beads in DMSO treated SW620 cells compared to DMSO treated HCT116 cells. Importantly, the amount of active TRIO was reduced upon low dose Taxol treatment. ITX3 treated cells exhibited the lowest proportion of active TRIO, demonstrating a significant inhibition of TRIO activity (Fig 3.11a).

GEF-assays were also performed using the melanoma cell lines SK-Mel-28 and SK-Mel-103. Here, it was distinguished between mitotic and interphase cells. Mitotic cells were obtained by treatment with $2 \mu \mathrm{M}$ DME for $16 \mathrm{~h}$. Additionally, cells were treated with DMSO, $0.5 \mathrm{nM}$ Taxol or $15 \mu \mathrm{M}$ ITX3. Interestingly, SK-Mel-103 cells displayed elevated levels of endogenous TRIO in comparison to SK-Mel-28 (depicted as input). These increased levels of endogenous TRIO were not altered upon Taxol or ITX3 treatment (Fig 3.11b). After immunoprecipitation using GST-Rac1 G15A agarose beads a higher proportion of active TRIO was detected in DMSO treated SK-Mel-103 when compared to DMSO treated SK-Mel-28 cells. This increased level of active TRIO was reduced by treatment with $0.5 \mathrm{nM}$ Taxol. ITX3 treated cells exhibited very low proportions of active TRIO (Fig 3.11b). 
a
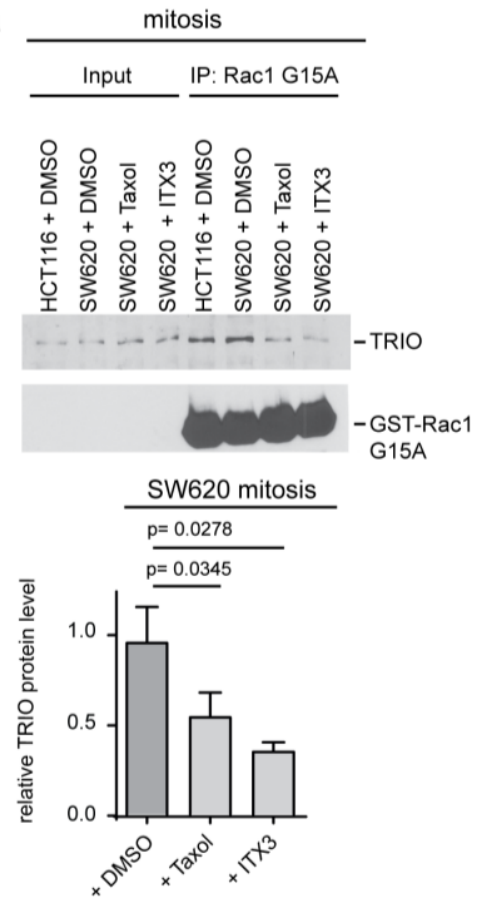

b
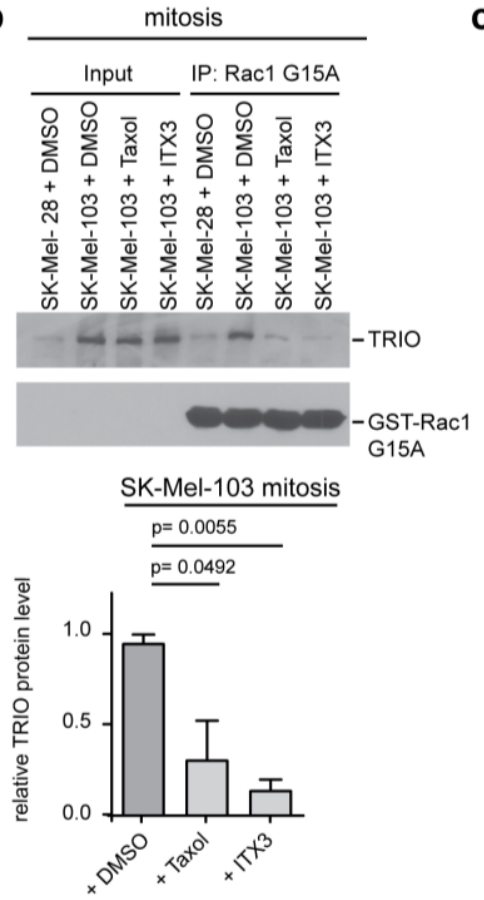

C

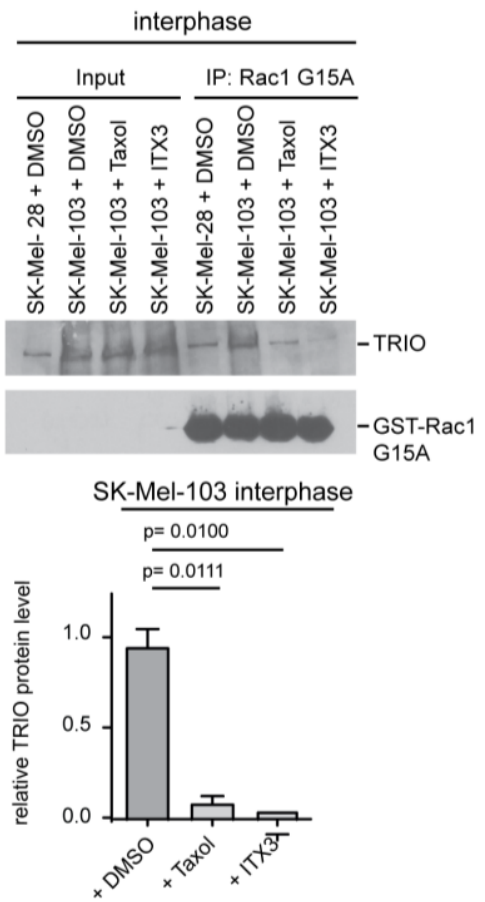

Figure 3.11: Elevated levels of active TRIO in SW620 and SK-Mel-103 cells depend on increased microtubule plus-end assembly rates. (a) HCT116 cells and SW620 cells were treated with $2 \mu \mathrm{M}$ DME for $16 \mathrm{~h}$ in combination with DMSO, $0.2 \mathrm{nM}$ Taxol or $15 \mu \mathrm{M}$ ITX3. GEF assays were performed using $2 \mathrm{mg}$ lysate and $10 \mu \mathrm{g}$ GST-Rac1 G15A agarose beads for $1 \mathrm{~h}$ at $4^{\circ} \mathrm{C}$. A representative western blot detecting TRIO is shown and quantifications of relative TRIO protein levels from three independent experiments are given. Bar graphs show amounts of active TRIO normalized to control treated cells (mean \pm sem, $t$-test, $n=3$ ). (b) SK-Mel-28 and SK-Mel-103 cells were treated with $2 \mu \mathrm{M}$ DME for $16 \mathrm{~h}$ in combination with DMSO, $0.5 \mathrm{nM}$ Taxol or $15 \mu \mathrm{M}$ ITX3. GEF assays were performed with $2 \mathrm{mg}$ lysate and $10 \mu \mathrm{g}$ GST-Rac $1 \mathrm{G} 15 \mathrm{~A}$ agarose beads for $1 \mathrm{~h}$ at $4^{\circ} \mathrm{C}$. A representative western blot detecting TRIO is shown and quantifications of relative TRIO protein levels from three independent experiments are given. Bar graphs show amounts of active TRIO normalized to control treated cells (mean \pm sem, $t$-test, $n=3$ ). (c) Asynchronously growing SK-Mel-28 and SK-Mel-103 cells were treated with DMSO, $0.5 \mathrm{nM}$ Taxol or $15 \mu \mathrm{M}$ ITX3 for $16 \mathrm{~h}$. GEF assays were performed using $2 \mathrm{mg}$ lysate and $10 \mu \mathrm{g}$ GST-Rac1 G15A agarose beads for $1 \mathrm{~h}$ at $4^{\circ} \mathrm{C}$. A representative western blot detecting TRIO is shown and quantifications of relative TRIO protein levels from three independent experiments are given. Bar graphs show proportions of active TRIO normalized to control treated cells (mean \pm sem, $t$-test, $n=3$ ).

SK-Mel-103 and SK-Mel-28 cells were also analyzed during interphase. Asynchronously growing cells were treated with DMSO, $0.5 \mathrm{nM}$ Taxol or $15 \mu \mathrm{M}$ ITX3 for $16 \mathrm{~h}$. Also during interphase, SK-Mel-103 cells exhibited increased proportions of endogenous TRIO as opposed to SK-Mel-28 cells (depicted as input) (Fig 3.11c). Higher proportions of active TRIO were immunoprecipitated using GST-Rac1 G15A agarose beads in DMSO treated SKMel-103 as opposed to DMSO treated SK-Mel-28 cells. These elevated proportions were reduced upon low dose Taxol and ITX3 treatment.

In summary, GEF assays revealed elevated levels of active TRIO in the CIN cell line SW620 during mitosis as well as in the highly migratory and invasive and cell line SK-Mel-103 in interphase and mitosis. GEF activity depended on increased microtubule plus-end assembly rates, since Taxol treatment decreased the proportion of active TRIO. 
TRIO was shown to bind to microtubule plus-ends via Nav1/EB1-complex or directly via EB1 (Van Haren et al. 2014). Therefore, it was tested, whether GEF activity of TRIO depends on its microtubule plus-end localization via EB1. SW620 and SK-Mel-103 cells were transfected with $80 \mathrm{pmol}$ LUCIFERASE or EB1 siRNA. To investigate active TRIO levels in mitotic SW620 cells, cells were treated with $2 \mu \mathrm{M}$ DME for $16 \mathrm{~h}$. GEF assays were performed and revealed decreased proportions of active TRIO upon EB1 repression in comparison to cells transfected with LUCIFEARSE siRNA (Fig. 3.12a). SK-Mel-103 cells were analyzed during mitosis and interphase. Mitotic cells were obtained by treatment with $2 \mu \mathrm{M} D \mathrm{DME}$ for $16 \mathrm{~h}$. During both, mitosis and interphase, SK-Mel-103 cells exhibited decreased proportions of active TRIO upon repression of EB1 (Fig. 3.12b-c), revealing an EB1-dependent activation of TRIO.

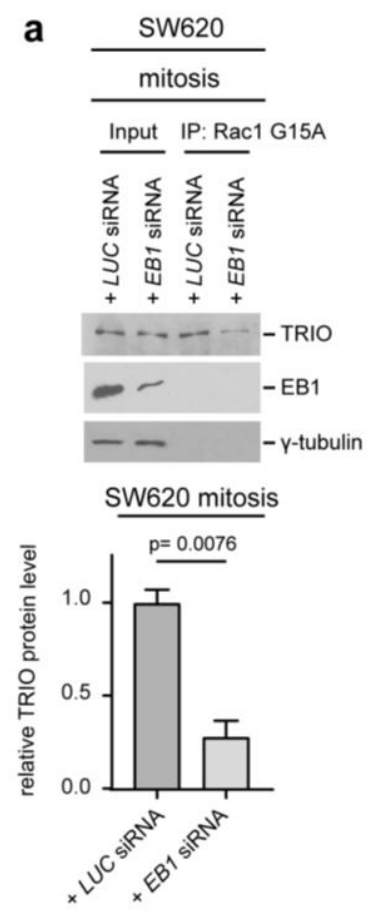

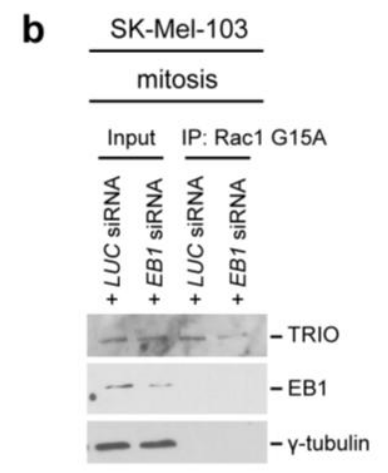

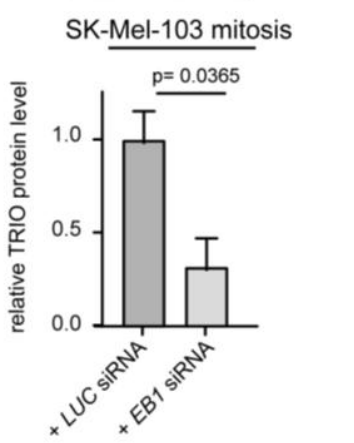

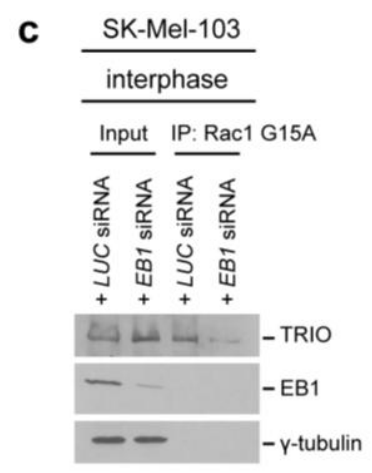

SK-Mel-103 interphase

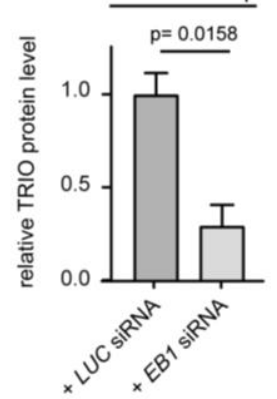

Figure 3.12: TRIO activity depends on its microtubule plus-end localization via EB1. (a) SW620 cells were transfected with 80 pmol LUCIFERASE or EB1 siRNA. Cells were treated with $2 \mu \mathrm{M}$ DME for $16 \mathrm{~h}$. GEF assays were performed using $2 \mathrm{mg}$ lysate and $10 \mu \mathrm{g}$ GST-Rac1 G15A agarose beads for $1 \mathrm{~h}$ at $4^{\circ} \mathrm{C}$. A representative western blot and quantifications of active TRIO protein levels are given. Bar graphs show proportions of active TRIO normalized to LUCIFERASE transfected cells (mean \pm sem, $t$-test, $\mathrm{n}=3$ ). (b) SK-Mel-103 cells were transfected with 80 pmol LUCIFERASE or EB1 siRNA. Cells were treated with $2 \mu \mathrm{M}$ DME for $16 \mathrm{~h}$. GEF assays were performed using $2 \mathrm{mg}$ lysate and $10 \mu \mathrm{g}$ GST-Rac1 G15A agarose beads for $1 \mathrm{~h}$ at $4^{\circ} \mathrm{C}$. A representative western blot and quantifications of active TRIO protein levels are given. Bar graphs show proportions of active TRIO normalized to LUCIFERASE transfected cells (mean \pm sem, $t$-test, $n=3$ ). (c) SK-Mel-103 cells were transfected with 80 pmol LUCIFERASE or EB1 siRNA. GEF assays were performed using $2 \mathrm{mg}$ lysate and $10 \mu \mathrm{g}$ GST-Rac1 G15A agarose beads for $1 \mathrm{~h}$ at $4^{\circ} \mathrm{C}$. A representative western blot and quantifications of active TRIO protein levels are shown. Bar graphs show proportions of active TRIO normalized to LUCIFERASE transfected cells (mean \pm sem, $t$-test, $\mathrm{n}=3$ ). 


\subsubsection{Inhibition Of TRIO Decreases CIN And Migration in SW620 and SK-Mel-103 Cells}

In order to analyze whether increased TRIO activity as detected in the GEF assays influence the migratory and the CIN phenotype, SK-Mel-103 and SW620 cells were treated with the TRIO specific inhibitor ITX3. ITX3 treatment did not alter the microtubule plus-end assembly rates during mitosis and interphase in neither SW620 nor SK-Mel-103 cells (Fig. 3.13a).

a

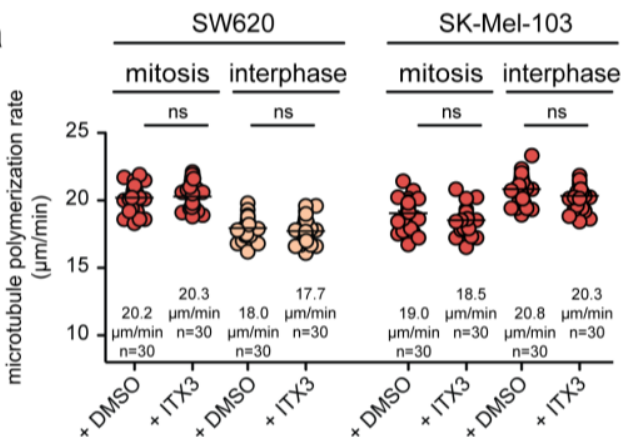

d

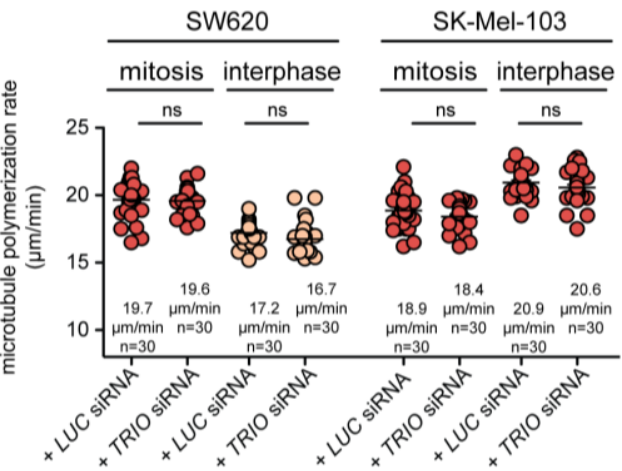

b

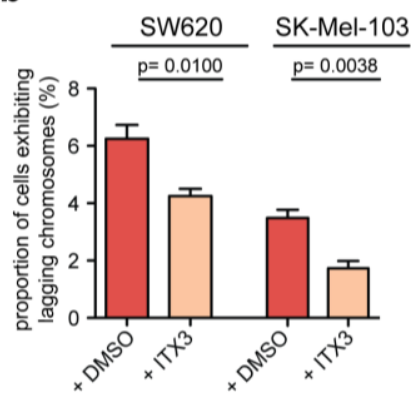

e

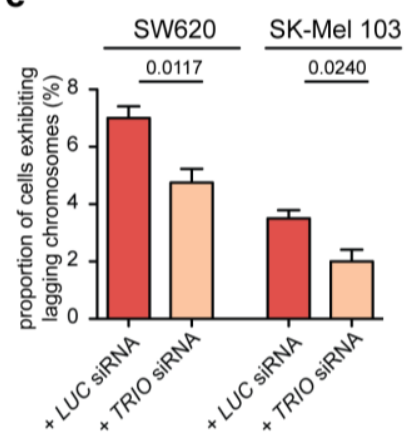

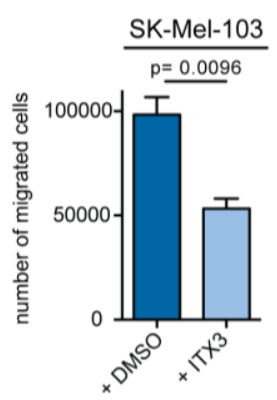

f

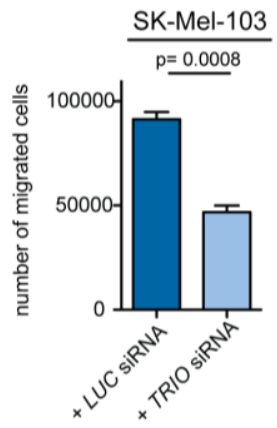

Figure 3.13: Inhibition of TRIO suppresses the generation of lagging chromosomes and inhibits cell migration. (a) Measurements of mitotic and interphase microtubule plus-end assembly rates in SW620 and SK-Mel-103 cells treated with DMSO or $15 \mu \mathrm{M}$ ITX3. Scatter dot plots show the average microtubule plus-end assembly rates of 20 microtubules per cell (mean \pm sem, $t$-test, $n=30$ cells from three independent experiments). (b) Quantification of cells exhibiting lagging chromosomes during anaphase. SW620 and SK-Mel-103 cells were treated with DMSO or $15 \mu \mathrm{M}$ ITX3. Cells were synchronized at the $\mathrm{G}_{1} / \mathrm{S}$ transition by double thymidine block and released into the cell cycle. After $8.5 \mathrm{~h}-9.5 \mathrm{~h}$ cells were fixed and stained for immunofluorescence microscopy. Bar graphs show quantification of cells showing at least one CREST-positive chromatid (mean values \pm sem, $t$-test, $n=4$ with a total of 400 anaphase cells ). (c) Transwell migration assays of SK-Mel-103 cells. Cells were treated with DMSO or $15 \mu \mathrm{M}$ ITX3. 200,000 cells were seeded into cell culture inserts. Bar graphs show the number of migrated cells after $24 \mathrm{~h}$ (mean \pm sem, $t$-test, $\mathrm{n}=3$ ). (d) Measurements of mitotic and interphase microtubule plus-end assembly rates in SW620 and SK-Mel-103 cells transfected with $60 \mathrm{pmol}$ LUCIFERASE or TRIO siRNA. Scatter dot plots show the average microtubule plus-end assembly rates of 20 microtubules per cell (mean \pm sem, $t$-test, $n=30$ cells from three independent experiments). (e) Quantification of cells showing lagging chromosomes during anaphase. SW620 and SK-Mel-103 cells were transfected with 60 pmol LUCIFERASE or TRIO siRNA. Cells were synchronized at the $G_{1} / S$ transition and released into the cell cycle. After $8.5 \mathrm{~h}-9.5 \mathrm{~h}$ cells were fixed and stained for immunofluorescence microscopy and the occurrence of lagging chromosomes was analyzed. Bar graphs show the amount of cells exhibiting at least one CREST-positive chromatid (mean values \pm sem, $t$-test, $n=4$ with a total of 400 anaphase cells). (f) Transwell migration assays of SK-Mel-103 cells. Cells were transfected with 60 pmol LUCIFERASE or TRIO siRNA. 200,000 cells were seeded and bar graphs show the proportion of migrated cells after $24 \mathrm{~h}$ (mean $\pm \mathrm{sem}, t$-test, $n=3)$. 
However, the proportion of cells showing lagging chromosomes was significantly reduced in both cell lines. In SW620 cells, the occurrence of lagging chromosomes was reduced from $6.25 \%$ to $4.25 \%$ on average, whereas in SK-Mel- 103 cells the amount was decreased from $3.5 \%$ to $1.75 \%$ on average (Fig. 3.13b). Transwell migration assays of SK-Mel-103 cells also revealed a decreased cell migration activity. Upon ITX3 treatment, the number of migrated cells was reduced from 98,333 cells to 53,333 cells (Fig. $3.13 c$ ).

To exclude unspecific effects of the TRIO inhibitor, siRNA-mediated repression of TRIO was conducted. These experiments confirmed the results obtained by ITX3 treatment. Although the microtubule plus-end assembly rates were not significantly altered during mitosis and interphase (Fig. 3.13d), a significant decrease in the occurrence of lagging chromosomes was detected in both SW620 and SK-Mel-103 cells upon TRIO repression. SW620 cells exhibited on average $4.75 \%$ lagging chromosomes, whereas LUCIFERASE transfected cells exhibited 7\%. In SK-Mel-103 cells, the amount of cells displaying lagging chromosomes was reduced from $3.5 \%$ to $2 \%$ on average (Fig. 3.13 e). In addition, SK-Mel-103 cells exhibited a reduced migration behavior upon TRIO repression compared to LUCIFERASE transfected cells. The amount of migrated cells was reduced from 91,333 cells to 46,667 cells (Fig. 3.13f). These results might indicate that increased TRIO activity can account for the induction of lagging chromosomes and increased cell migration.

\subsubsection{Elevated Rac1 Activity Affects CIN And Migration In SW620 And SK-Mel-103 Cells}

The previous experiments revealed an increased level of active TRIO in the CIN cell line SW620 during mitosis and in the highly migratory and invasive cell line SK-Mel-103. Since TRIO functions as a GEF for Rac1 (Blangy et al. 2000; Cannet et al. 2014), it was of interest to investigate whether Rac1 activity was increased in these cells. To test this, PAK-PBD agarose beads were used for pull down assays to isolate GTP-bound Rac1 from cell lysates derived from mitotic HCT116 and SW620 cells and from mitotic and interphase SK-Mel-28 and SK-Mel-103 cells.

HCT116 and SW620 cells were synchronized in mitosis by treatment with $2 \mu \mathrm{M}$ DME for $16 \mathrm{~h}$. The treatment was combined with DMSO, $0.2 \mathrm{nM}$ Taxol, $15 \mu \mathrm{M}$ ITX3 or $40 \mu \mathrm{M}$ of the Rac1 inhibitor NSC23766. While the overall Rac1 levels did not vary significantly between the two cell lines, DMSO treated SW620 cells displayed nearly 1.5 fold higher levels of active Rac1 as opposed to DMSO treated HCT116 cells. Importantly, Taxol treatment of SW620 cells reduced Rac1 activity to levels similar to HCT116 cells. The inhibition of Rac1 by using its specific inhibitor NSC23766 served as a control and revealed a significant inhibition of 
Rac1, whereas treatment with the TRIO inhibitor ITX3 revealed the dependency of Rac1 on TRIO (Fig. 3.14a).

Rac1 pull down assays were also performed with mitotic and interphase SK-Mel-28 and SK-Mel-103 cells. To obtain mitotic lysates, SK-Mel-28 and SK-Mel-103 cells were treated with $2 \mu \mathrm{M}$ DME for $16 \mathrm{~h}$ in combination with DMSO, $0.5 \mathrm{nM}$ Taxol, $40 \mu \mathrm{M}$ NSC23766 or 15 $\mu \mathrm{M}$ ITX3. Rac1 activity assays using mitotic cell lysates revealed nearly 1.5 -fold increased levels of active Rac1 in DMSO treated SK-Mel-103 cells compared to DMSO treated SK-Mel-28 cells, whereas the overall Rac1 levels did not vary between the two cell lines. Similar to the experiment using SW620 cells, treatment with low doses Taxol, NSC23766 and ITX3 reduced the proportion of active Rac1 also in the melanoma cells (Fig. 3.14b).

a
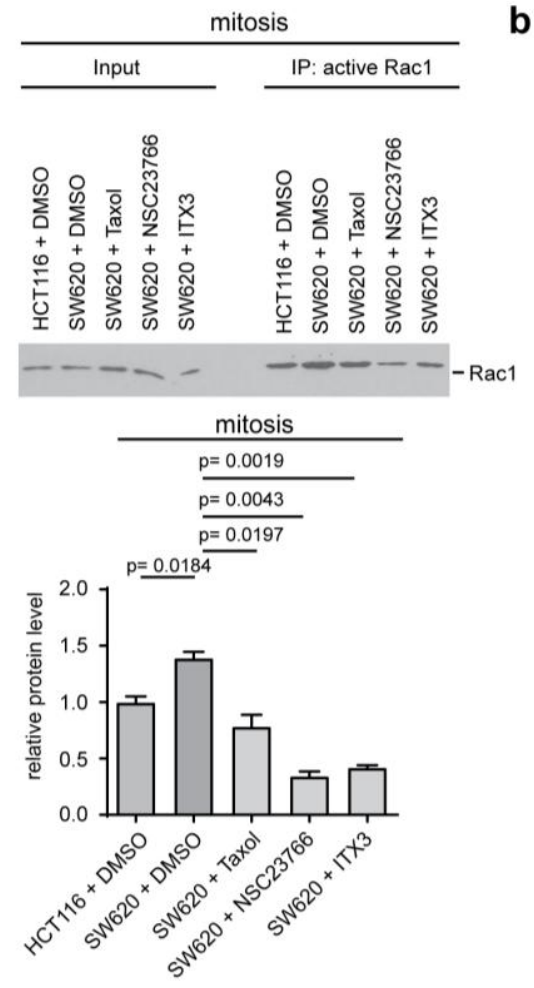

b

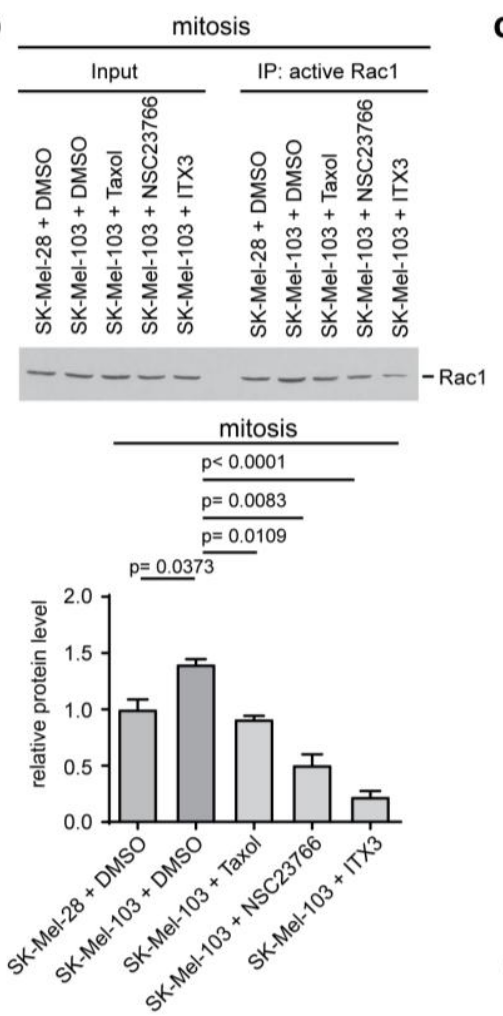

C
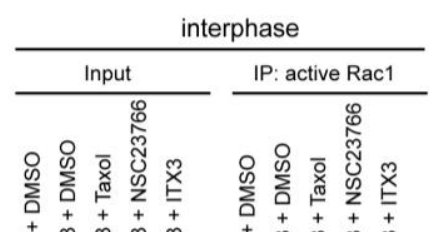

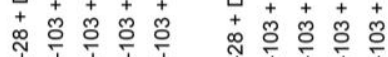

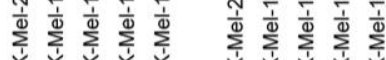

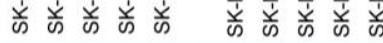

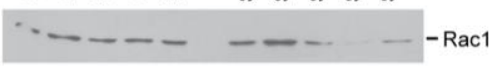

interphase

$p=0.0157$

$p=0.0499$ $\mathrm{p}=0.0748$ $p=0.0312$

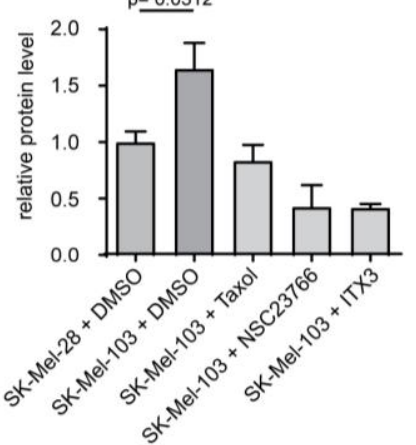

Figure 3.14: Elevated levels of active Rac1 are dependent on increased microtubule plus-end assembly rates. (a) HCT116 cells were treated with DMSO and SW620 cells were treated with DMSO, $0.2 \mathrm{nM}$ Taxol, $40 \mu \mathrm{M}$ NSC23766 or $15 \mu \mathrm{M}$ ITX3 for $16 \mathrm{~h}$ combined with $2 \mu \mathrm{M}$ DME to synchronize cells in mitosis. $1.5 \mathrm{mg}$ of cell lysates were incubated with $10 \mu \mathrm{g}$ PAK-PBD agarose beads for $30 \mathrm{~min}$ at $4^{\circ} \mathrm{C}$ and active Rac1 was precipitated and detected on western blot using Rac1 specific antibodies. A representative western blot is shown. Western blot quantifications of active Rac1 protein levels normalized to DMSO treated control cells are represented as bar graphs (mean \pm sem, $t$-test, $\mathrm{n}=3$ ). (b) SK-Mel-28 and SK-Mel-103 cells were treated with $2 \mu \mathrm{M}$ for $16 \mathrm{~h}$ combined with DMSO, $0.5 \mathrm{nM}$ Taxol, $40 \mu \mathrm{M}$ NSC23766 or $15 \mu \mathrm{M}$ ITX3 and processed as HCT116 and SW620 described in (a). A representative western blot is shown. Western blot quantifications of active Rac1 protein levels normalized to DMSO treated control cells are represented as bar graphs (mean \pm sem, $t$-test, $n=3$ ). (c) Asynchronously growing SK-Mel-28 cells were treated with DMSO and SK-Mel-103 cells were treated with DMSO, $0.5 \mathrm{nM}$ Taxol, $40 \mu \mathrm{M}$ NSC23766 or $15 \mu \mathrm{M}$ ITX3 for $16 \mathrm{~h}$. Interphase cell lysates were processed as described in (a). A representative western blot is shown. Western blot quantifications of active Rac1 protein levels normalized to DMSO treated control cells are represented as bar graphs (mean \pm sem, $t$-test, $n=3$ ). 
SK-Mel-28 and SK-Mel-103 cells were also analyzed regarding their proportions of active Rac1 during interphase. Similar to mitotic cells, DMSO treated interphase SK-Mel-103 cells exhibited higher levels of active Rac1 as opposed to DMSO treated SK-Mel-28 cells. Here, the level of active Rac1 was increased approximately 1.75-fold. Also in interphase, these elevated Rac1 levels could be reduced by Taxol, as well as by NSC23766 and ITX3 treatment (Fig 3.14c).

Active Rac1 pull-down assays revealed elevated levels of active Rac1 in SW620 cells during mitosis and in SK-Mel-103 cells during both, mitosis and interphase. Since ITX3 treatment reduced the levels of active Rac1, Rac1 was verified as a downstream target of TRIO.

In order to further evaluate the relevance of active Rac1 for the migrating and invasive phenotype as well as for the generation of lagging chromosomes, a constitutively active mutant of Rac1 (Rac1 Q61L) was overexpressed in HCT116 cells. HCT116 cells exhibit neither a migratory phenotype nor elevated amounts of cells with lagging chromosomes. However, after overexpression of Rac1 Q61L, the migration potential of HCT116 cells was enhanced from 35,667 cells to 69,000 cells on average. Treatment with the Rac1 inhibitor NSC23766 reduced cell migration observed upon Rac Q61L overexpression to a level comparable to the control transfected cells, showing the specificity of the Rac1 Q61L overexpression (Fig. 3.15a).
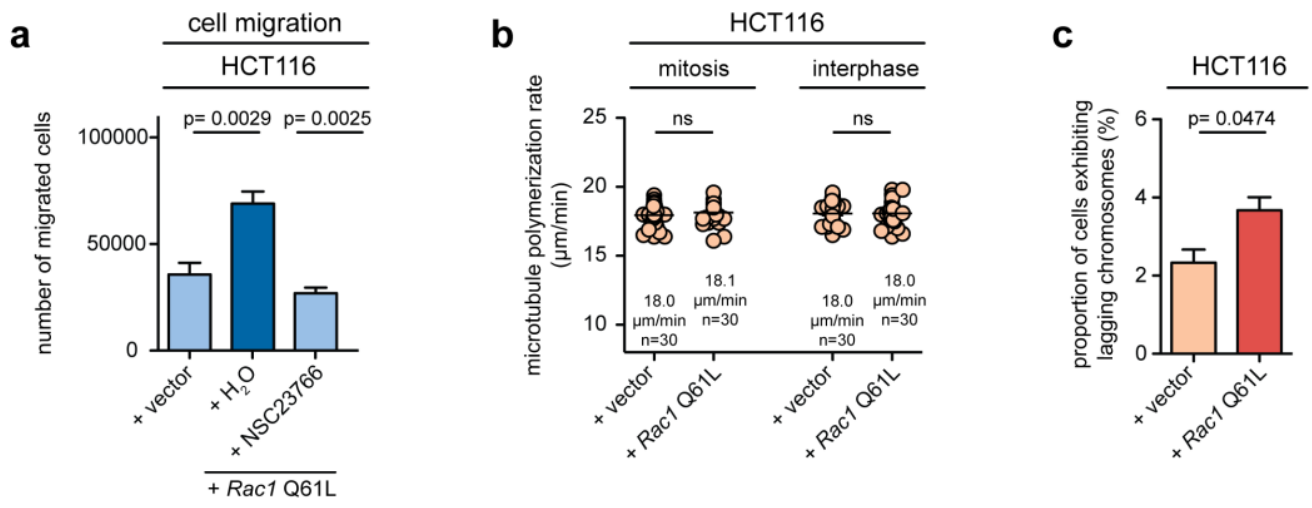

Figure 3.15: Overexpression of a constitutively active Rac1 mutant protein induces migration and the generation of lagging chromosomes. (a) Transwell migration assays of HCT116 cells transfected with a constitutively active mutant of Rac1 (Rac1 Q61L).10 $\mu \mathrm{g}$ Rac1 Q61L were overexpressed and cells were treated with $\mathrm{H}_{2} \mathrm{O}$ or $40 \mu \mathrm{M}$ NSC23766. 200,000 cells were seeded for the migration assay. Bar graphs show the number of migrated cells after $24 \mathrm{~h}$ (mean \pm sem, $t$-test, $\mathrm{n}=3$ ). (b) Measurements of mitotic and interphase microtubule plus-end assembly rates in HCT116 cells with an overexpression of Rac1 Q61L. $1 \mathrm{~h}$ prior to EB3 tracking experiments, cells were treated with $2 \mu \mathrm{M}$ DME. Scatter dot plots show the average microtubule plus-end assembly rates of 20 microtubules per cell (mean \pm sem, $t$-test, $n=30$ cells from three independent experiments). (c) Quantification of HCT116 cells displaying lagging chromosomes during anaphase after overexpression of Rac1 Q61L. Cells were synchronized at $\mathrm{G}_{1} / \mathrm{S}$ transition by double thymidine block and released into the cell cycle. After $8.5 \mathrm{~h}$ cells were fixed and stained for immunofluorescence microscopy and cells with lagging chromosomes were quantified. Bar graphs show the amount of HCT116 cells exhibiting at least one CREST-positive chromatid (mean values $\pm s e m$, $t$-test, $n=3$ with a total of 300 anaphase cells). 
Interestingly, EB3 tracking experiments revealed that the overexpression of Rac1 Q61L did not affect microtubule plus-end assembly rates (Fig. 3.15b). However, the quantification of cells with lagging chromosomes during anaphase showed increased amounts from $2.3 \%$ in control vector transfected cells to 3.6\% in Rac1 Q61L expressing cells (Fig. 3.15c).

Since the overexpression of a constitutively active Rac1 mutant induced a migratory phenotype and the generation of lagging chromosomes, the question arose whether a dominant negative mutant of Rac1 would reduce migration and the amount of cells showing lagging chromosomes in migratory and CIN cells. To test this, a dominant negative mutant of Rac1 (Rac1 T17N), which is described to inhibit Rac1's function (Ridley et al. 1992), was overexpressed in SK-Mel-103 and SW620 cells. In transwell migration assays, the overexpression of Rac1 $\mathrm{T} 17 \mathrm{~N}$ indeed led to a decrease of the migration potential. Upon control vector transfection, 95,417 migrating cells were detected on average, which were reduced to 51,667 cells upon overexpression of Rac1 T17N (Fig. 3.16a). While having no significant influence onto the microtubule plus-end assembly rates of SW620 and SK-Mel-103 cells (Fig. 3.16b), the amount of cells exhibiting lagging chromosomes was significantly decreased (Fig. 3.16c). Control transfected SK-Mel-103 cells exhibited 4\% lagging chromosomes on average, which were reduced to $2.25 \%$ upon overexpression of Rac1 T17N. In SW620 cells, the occurrence of lagging chromosomes was reduced from $6.7 \%$ to $3.3 \%$.
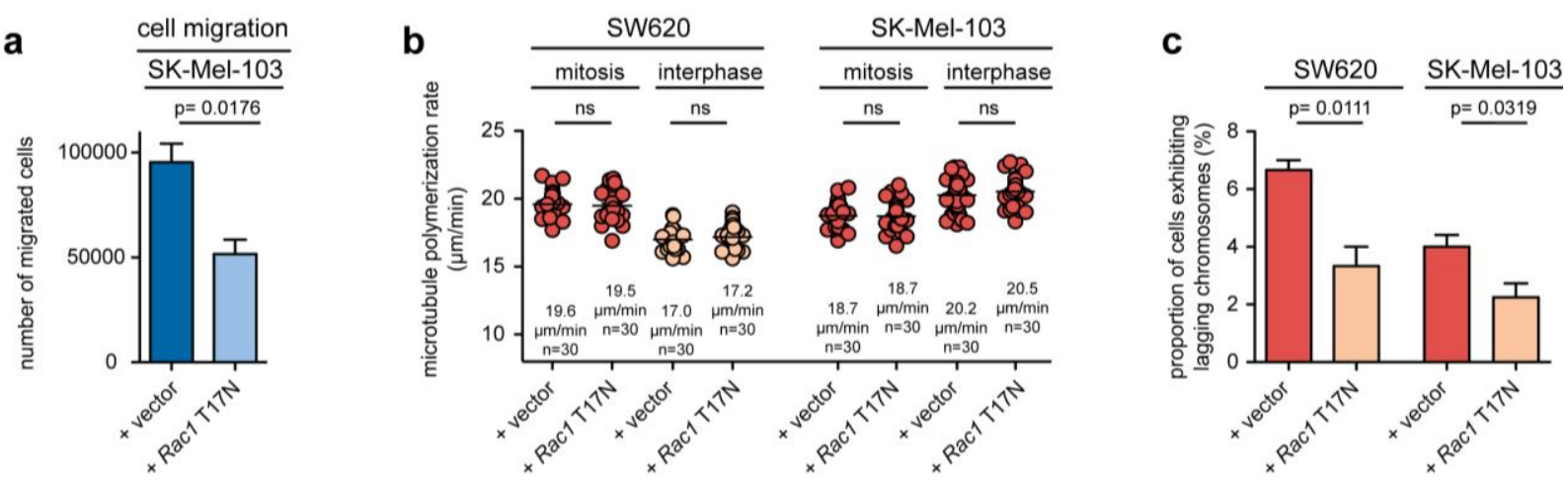

Figure 3.16: Overexpression of a dominant negative mutant protein of Rac1 reduces migration and the generation of lagging chromosomes in SW620 and SK-Mel-103 cells. (a) Transwell migration assays of SK-Mel-103 cells transfected with Rac1 T17N.10 $\mu$ g Rac1 T17N or control vector were overexpressed. 200,000 cells were seeded in cell culture inserts for migration assay. Bar graphs show the amount of migrated cells after $24 \mathrm{~h}$ (mean \pm sem, $t$-test, $\mathrm{n}=3$ ). (b) Measurements of mitotic and interphase microtubule plus-end assembly rates in SW620 and SK-Mel-103 cells with an overexpression of Rac1 T17N. $1 \mathrm{~h}-2 \mathrm{~h}$ prior to EB3 tracking experiments, cells were treated with $2 \mu \mathrm{M}$ DME. Scatter dot plots show the average microtubule plus-end assembly rates of 20 microtubules per cell (mean \pm sem, $t$-test, $n=30$ cells from three independent experiments). (c) Quantification of cells showing lagging chromosomes during anaphase in SW620 and SK-Mel-103 cells with an overexpression of Rac1 T17N. Cells were synchronized at $\mathrm{G}_{1} / \mathrm{S}$ transition by double thymidine block and released into the cell cycle. After $8.5 \mathrm{~h}-9.5 \mathrm{~h}$ cells were fixed and stained for immunofluorescence microscopy. Bar graphs show the amount of cells exhibiting at least one CREST-positive signal (mean \pm sem, $t$-test, $n=3$ (SW620) -4 (SK-Mel-103) with a total of 300-400 anaphase cells). 
To further substantiate the role of Rac1 in cell migration and in the generation of lagging chromosomes, Rac1 activity was inhibited in SW620 and SK-Mel-103 cells by treatment with the Rac1 specific inhibitor NSC23766. In transwell migration assays, NSC23766 treatment led to a significant decrease of migrating SK-Mel-103 cells. In comparison to control treated cells, the amount of migrated cells was reduced from 93,250 cells to 44,333 cells on average upon NSC23766 treatment (Fig. 3.17a). In addition, analyses of lagging chromosomes in SW620 and SK-Mel-103 cells revealed a decreased number of cells displaying lagging chromosomes after treatment with NSC23766. The occurrence of lagging chromosomes was reduced from $6.3 \%$ to $3.3 \%$ and $4.3 \%$ to $2 \%$ in SW620 and SK-Mel-103 cells, respectively (Fig. 3.17b). However, microtubule plus-end assembly rates were neither altered in interphase nor in mitosis in both cell lines (Fig. 3.17c).
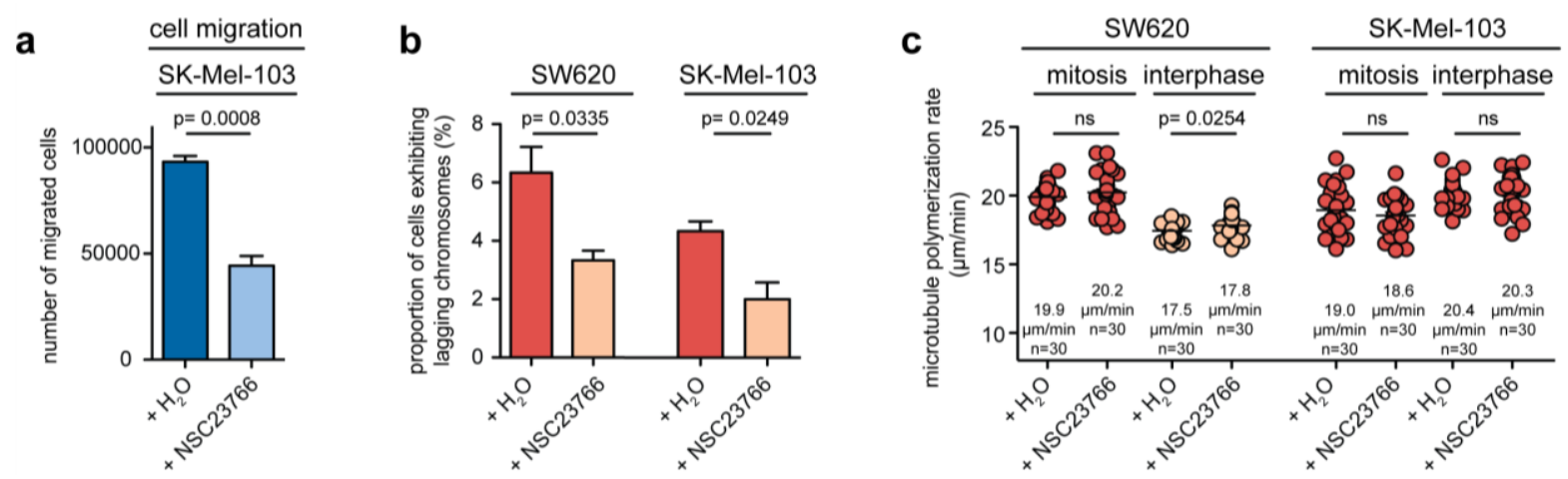

Figure 3.17: Inhibition of Rac1 by its specific inhibitor decreases migration and the generation of lagging chromosomes. (a) Transwell migration assays of SK-Mel-103 cells treated with $\mathrm{H}_{2} \mathrm{O}$ or $40 \mu \mathrm{M}$ NSC23766. 200,000 cells were seeded for the migration assay. Bar graphs show the number of migrated cells after $24 \mathrm{~h}$ (mean \pm sem, $t$-test, $\mathrm{n}=3$ ). (b) Quantification of cells showing lagging chromosomes during anaphase in SW620 and SK-Mel-103 cells treated with $\mathrm{H}_{2} \mathrm{O}$ or $40 \mu \mathrm{M}$ NSC23766. Cells were synchronized by double thymidine block and released into the cell cycle. After $8.5 \mathrm{~h}-9.5 \mathrm{~h}$, cells were fixed and stained for immunofluorescence microscopy. Bar graphs show the proportion of cells exhibiting at least one CREST-positive chromatid (mean \pm sem, $t$-test, $n=3$ with a total of 300 anaphase cells). (c) Measurements of mitotic and interphase microtubule plus-end assembly rates in SW620 and SK-Mel-103 cells. Cells were treated with $\mathrm{H}_{2} \mathrm{O}$ or $40 \mu \mathrm{M}$ NSC23766 for $2 \mathrm{~h} .1-2 \mathrm{~h}$ prior to EB3 tracking experiments, cells were treated with $2 \mu \mathrm{M}$ DME. Scatter dot plots show the average microtubule plus-end assembly rates of 20 microtubules per cell (mean \pm sem, $t$-test, $\mathrm{n}=30$ cells from three independent experiments).

These results substantiate the hypothesis, that elevated levels of Rac1 contribute to both, a migratory phenotype and the generation of lagging chromosomes, in highly migratory cells exhibiting CIN. 


\subsubsection{The Arp2/3 Complex Acts Downstream Of Rac1 During Migration And The Development Of CIN}

Rac1 is well described for its role in lamellipodia formation during cell motility. In the lamellipodium, actin branching is achieved by Rac1-mediated activation of the Arp2/3 complex (Ridley et al. 2003). Therefore, it was of interest to investigate whether the Arp2/3 complex acts as a downstream effector of Rac1 during migration and chromosome segregation.

To address this, inhibition of Arp2/3 was achieved by using the specific Arp2/3 inhibitor CK666 in SW620 and SK-Mel-103 cells. Transwell migration assays using SK-Mel-103 cells were performed and revealed a significantly decreased amount of migrated cells upon CK666 treatment. The number of migrated SK-Mel-103 cells was reduced from 89,667 cells on average in control experiments to 58,167 migrated cells upon CK666 treatment

(Fig. 3.18a). Analyses of cells displaying lagging chromosomes revealed a significant decrease of chromosome missegregation in both cell lines. In CK666 treated SW620 cells, the amount of cells showing lagging chromosomes was reduced from $6 \%$ to $3.6 \%$ on average, whereas CK666 treatment in SK-Mel-103 cells decreased the number from $4.3 \%$ to 2.3\% (Fig. 3.18b). Similar to treatments with NSC23766 and ITX3, CK666 treatment had no significant influence on the microtubule plus-end assembly rates during mitosis and interphase in SW620 and SK-Mel-103 cells (Fig. 3.18c).
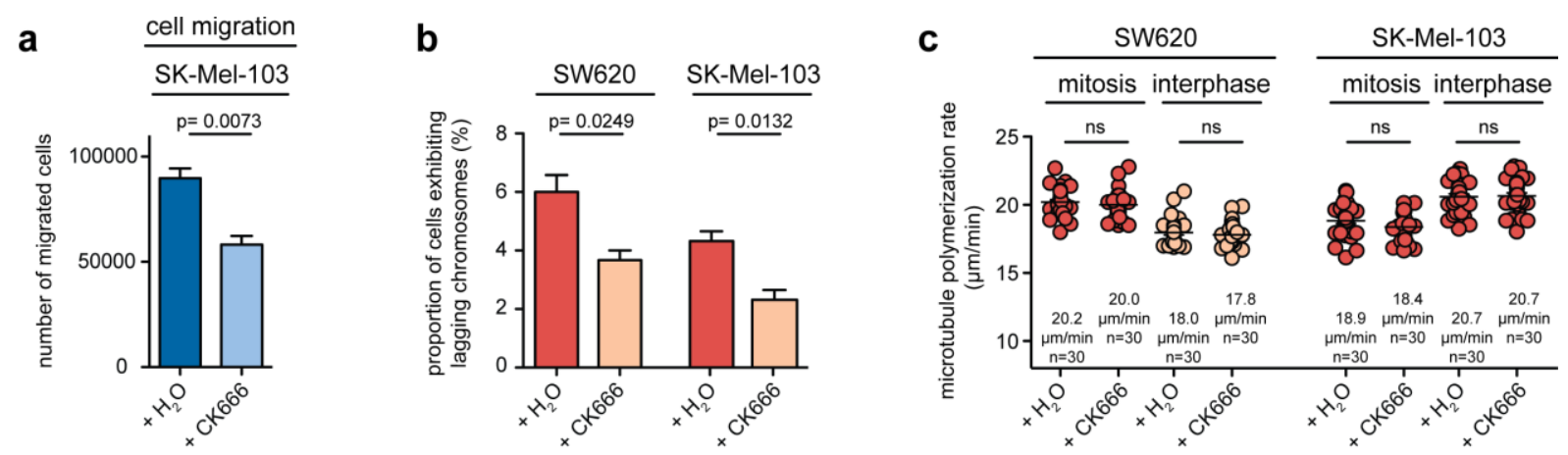

Figure 3.18: Inhibition of the Arp2/3 complex decreases migration and the generation of lagging chromosomes. (a) Transwell migration assays of SK-Mel-103 cells treated with $\mathrm{H}_{2} \mathrm{O}$ or 20 MM CK666. 200,000 cells were seeded in cell culture inserts for the migration assay. Bar graphs show the number of migrated cells after $24 \mathrm{~h}$ (mean \pm sem, $t$-test, $\mathrm{n}=3$ ). (b) Quantification of cells exhibiting lagging chromosomes during anaphase in SW620 and SK-Mel-103 cells treated with $\mathrm{H}_{2} \mathrm{O}$ or $20 \mu \mathrm{M}$ CK666. Cells were synchronized by double thymidine block and released into the cell cycle. After $8.5 \mathrm{~h}-9.5 \mathrm{~h}$, cells were fixed and stained for immunofluorescence microscopy. Bar graphs show the proportion of cells exhibiting at least one CREST-positive chromatid (mean \pm sem, $t$-test, $n=3$ with a total of 300 anaphase cells). (c) Measurements of mitotic and interphase microtubule plus-end assembly rates in SW620 and SK-Mel-103 cells. Cells were treated with DMSO or $20 \mu \mathrm{M}$ CK666 for $2 \mathrm{~h} .1-2 \mathrm{~h}$ prior to EB3 tracking experiments, cells were treated with $2 \mu \mathrm{M}$ DME. Scatter dot plots show the average microtubule plus-end assembly rates of 20 microtubules per cell (mean $\pm \mathrm{sem}, t$-test, $\mathrm{n}=30$ cells from three independent experiments). 
Since the inhibition of the Arp2/3 complex revealed similar results as previously obtained by Rac1 and TRIO inhibition, it seems likely, that TRIO, Rac1 and the Arp2/3 complex act in the same pathway. All three proteins seem to be important players for the development of a highly migratory phenotype and for the generation of lagging chromosomes. On the other hand, inhibition of any component of the TRIO-Rac1-Arp2/3 pathway resulted in decreased migration and decreased chromosome missegregation in highly migratory and invasive cancer cells exhibiting CIN.

\subsubsection{Inhibition Of TRIO, Rac1 And The Arp2/3 Complex Affects Spindle Orientation In SW620 Cells}

Ertych et al. described the phenotype of increased microtubule plus-end assembly rates and showed that these increased microtubule plus-end assembly rates are associated with transient mitotic spindle orientation defects, which, in turn, result in the induction of lagging chromosomes (Ertych et al. 2014). Since inhibition of TRIO, Rac1 and the Arp2/3 complex reduced the amounts of lagging chromosomes, I investigated, whether spindle orientation is influenced by the TRIO-Rac1-Arp2/3 pathway. To address this question, SW620 cells were seeded onto fibronectin coated slides and treated with DMSO, $0.2 \mathrm{nM}$ Taxol, $15 \mu \mathrm{M}$ ITX3, $40 \mu \mathrm{M}$ NSC23766 or $20 \mu \mathrm{M}$ CK666 for $16 \mathrm{~h}$. Afterwards, cells were fixed for immunofluorescence microscopy and the spindle axis angle was determined. Staining for $\alpha$-tubulin, $\mathrm{\gamma}$-tubulin and DAPI marked the mitotic spindle and the centrosomes and allowed the discrimination between cells in prometaphase and cells in metaphase due to positioning of the centrosomes with respect to the chromosomes (Fig. 3.19a). Images with a z-optical spacing of $0.4 \mu \mathrm{m}$ were taken and the spindle axis angle was determined by the formula: spindle axis angle $=$ (number of $z$-stacks ${ }^{*} 0.4 \mu \mathrm{m} /$ distance of centrosomes) ${ }^{*} 180 / \pi$ (Fig. 3.19b).

During prometaphase, DMSO treated SW620 cells exhibited a mean spindle axis angle of $22.8^{\circ}$, which was significantly reduced to $9.2^{\circ}$ upon treatment with $0.2 \mathrm{nM}$ Taxol. Similarly, inhibition of TRIO, Rac1 or the Arp2/3 complex significantly decreased the spindle axis angle. Upon drug treatment, mean spindle axis angles were reduced to values ranging from $10.1^{\circ}$ to $10.9^{\circ}$ (Fig. 3.19c). During metaphase, the spindle axis angle in DMSO treated SW620 cells was not as variable as observed in prometaphase cells. While prometaphase cells exhibited $22.8^{\circ}$, the analyses of metaphase cells revealed a mean spindle axis angle of $8.5^{\circ}$. However, a reduction of the mean spindle axis angle in metaphases was also detected upon inhibitor treatment. Taxol treatment reduced the angle in metaphase to $4.6^{\circ}$. Upon ITX3, NSC23766 and CK666 treatment, the spindle axis angles were reduced to $4.4^{\circ}, 5.4^{\circ}$ and $4.2^{\circ}$, respectively (Fig. $3.19 \mathrm{~d}$ ). 
a

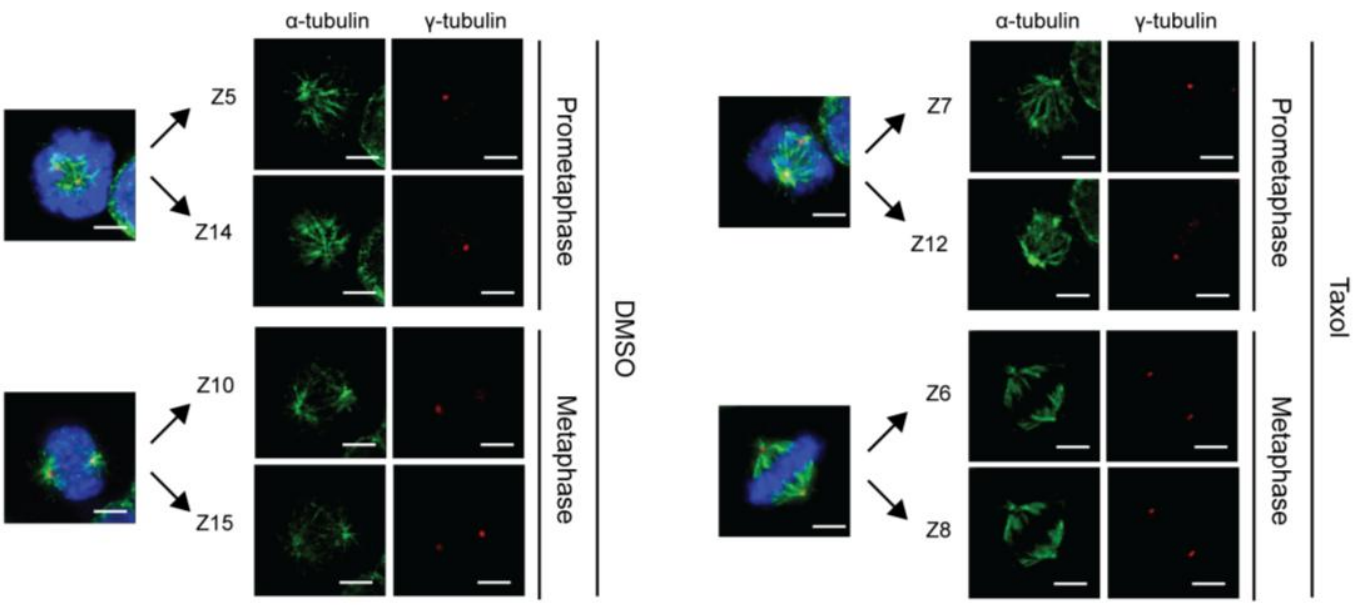

b

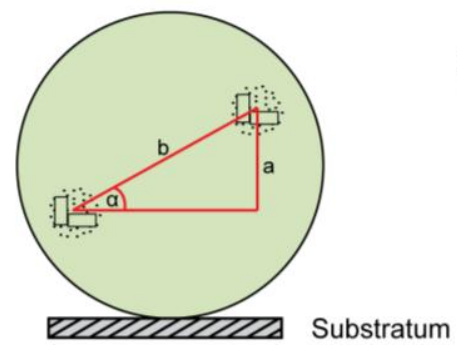

centrosome

$\alpha=$ spindle axis angle

$\mathrm{a}=$ height in $\mu \mathrm{m}$ (number of $\mathrm{z}$-stacks ${ }^{\star} 0.4 \mu \mathrm{m}$ )

$\mathrm{b}=$ distance between centrosomes in $\mu \mathrm{m}$

$\alpha=(a / b)^{\star} 180 / \pi$

c

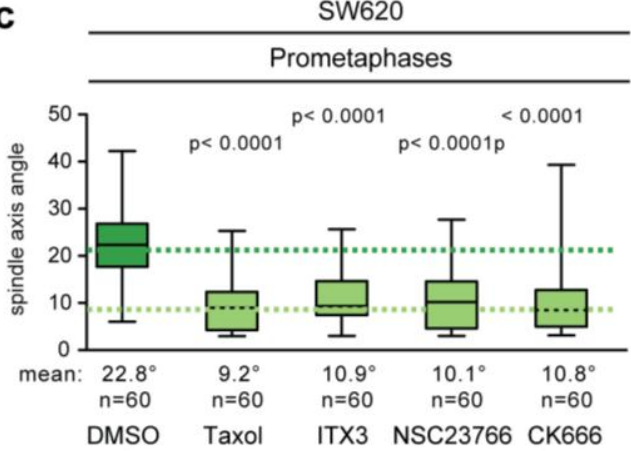

d

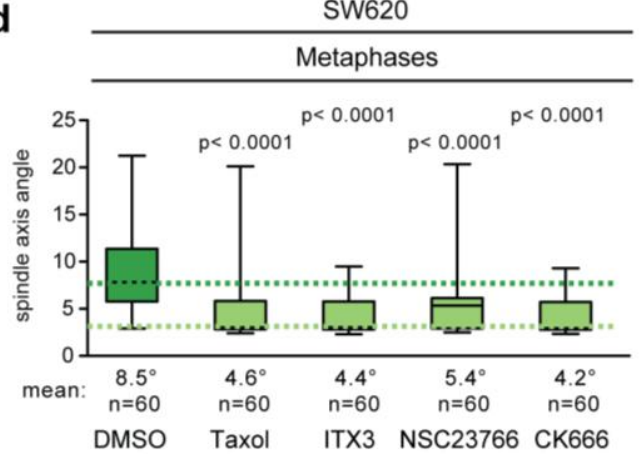

Figure 3.19: Analyses of spindle orientation in SW620 cells during prometaphase or metaphase. (a) Representative immunofluorescence images of DMSO or Taxol treated prometaphase or metaphase SW620 cells. Cells were stained for $\alpha$-tubulin, $y$-tubulin and DAPI. A maximum intensity projection as well as pictures with focused centrosomes are shown. $Z$ refers to the respective z-stack with focused centrosome. Scale bar $10 \mu \mathrm{M}$. (b) Schematic illustration for the determination of the spindle axis angle. (c) Measurements of spindle axis angle in prometaphase. SW620 cells were seeded onto fibronectin coated slides and treated with DMSO, $0.2 \mathrm{nM}$ Taxol, $15 \mu \mathrm{M}$ ITX3, $40 \mu \mathrm{M}$ NSC23766 or $20 \mu \mathrm{M}$ CK666 for $16 \mathrm{~h}$. Afterwards, cells were fixed and stained for immunofluorescence microscopy. Pictures of cells in prometaphase were taken and the angle between centrosomes and substratum was analyzed. The box and whisker plots show the range, median and quartile of the measurements ( $t$-test, $n=60$ cells from three independent experiments). Additionally, mean values are given. (d) Measurements of spindle axis angle in metaphase. SW620 cells were treated as described in (a). Pictures of cells in metaphase were taken and the angle between centrosomes and substratum was determined. The box and whisker plots show the range, mean and quartile of the measurements ( $t$-test, $n=60$ cells from three independent experiments). Additionally, mean values are given.

These results indicate an important role for TRIO, Rac1 and the Arp2/3 complex in spindle positioning during mitosis, in addition to their well established role in cell motility. 


\subsubsection{Inhibition Of TRIO, Rac1 Or The Arp2/3 Complex Suppresses CIN In SW620 And SK-Mel-103 Cells}

SK-Mel-103 and SW620 cells exhibited an increased amount of cells displaying lagging chromosomes, which were reduced by treatment with the TRIO inhibitor ITX3, the Rac1 inhibitor NSC23766 and the Arp2/3 complex inhibitor CK666. Since the occurrence of lagging chromosomes is a widely recognized mechanism for the appearance of CIN (Cimini et al. 2001), it was of interest to investigate whether the inhibition of TRIO, Rac1 and the Arp2/3 complex would also affect the CIN phenotype present in SK-Mel-103 and SW620 cells. To evaluate CIN, single cell clones of both cell lines were generated in the presence or absence of the different inhibitors. As a control, cells were treated with low doses of Taxol, because Taxol was already proven to suppress CIN (Ertych et al. 2014). Cell clones were grown for 30 generations and their chromosome number deviation was analyzed by chromosome counting in metaphase spreads and by CEP-FISH analyses. SW620 cell clones were analyzed by individual chromosome counting. Upon Taxol treatment, the amount of cells with a chromosome numbers deviating from modal was highly decreased (Fig. 3.20a).

a

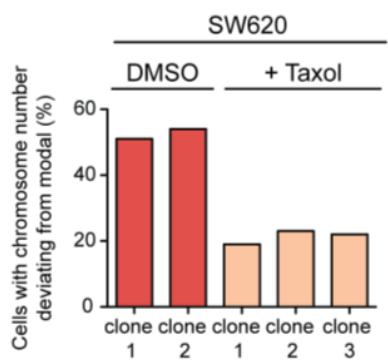

C

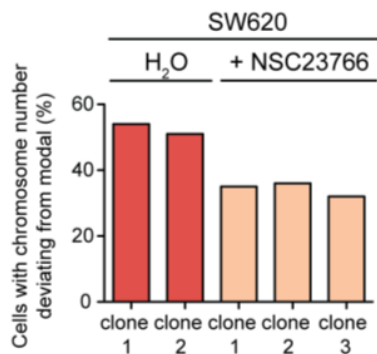

b

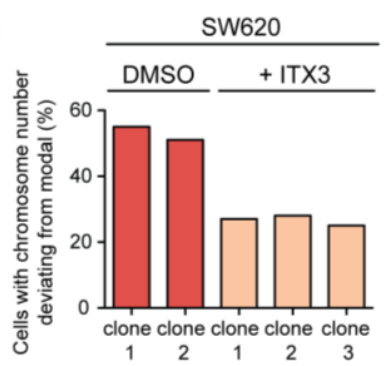

d

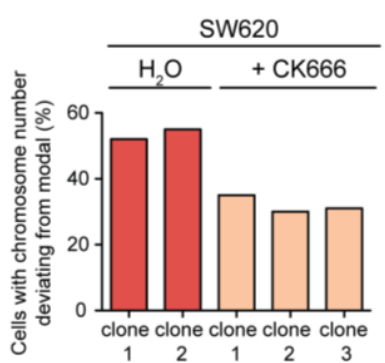

Figure 3.20: Inhibition of TRIO, Rac1 or the Arp2/3 complex suppresses CIN in SW620 cells. (a) Karyotype analyses by chromosome counting in metaphase spreads of single cell clones derived from SW620 cells treated with DMSO or $0.2 \mathrm{nM}$ Taxol for 30 generations. The percentage of cells with a chromosome number deviating from modal was determined ( $n=100$ cells). (b) Karyotype analyses by chromosome counting in metaphase spreads of single cell clones derived from SW620 cells treated with DMSO or $15 \mu \mathrm{M}$ ITX3 for 30 generations. The percentage of cells with a chromosome number deviating from modal was determined ( $n=100$ cells). (c) Karyotype analyses by chromosome counting in metaphase spreads of single cell clones treated with $\mathrm{H}_{2} \mathrm{O}$ or $40 \mu \mathrm{M}$ NSC23766 for 30 generations. The percentage of cells with a chromosome number deviating from modal was determined ( $n=100$ cells). (d) Karyotype analyses by chromosome counting in metaphase spreads of single cell clones derived from SW620 cells treated with $\mathrm{H}_{2} \mathrm{O}$ or $20 \mu \mathrm{M}$ CK666 for 30 generations. The percentage of cells with a chromosome number deviating from modal was determined ( $n=100$ cells). 
Also ITX3, NSC23677 and CK666 treated single cell clones showed decreased numbers of aneuploid cells when compared to the respective control treated cells (Fig. 3.20b-d). Thereby, inhibition of TRIO by ITX3 revealed the strongest reduction in cells with chromosome numbers deviating from modal.

SW620 single cell clones were further studied by CEP-FISH analyses. Therefore, $\alpha$-satellite probes specific for chromosome 7 and chromosome 15 were used.
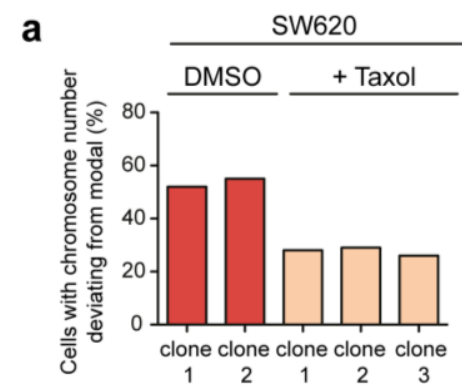

C

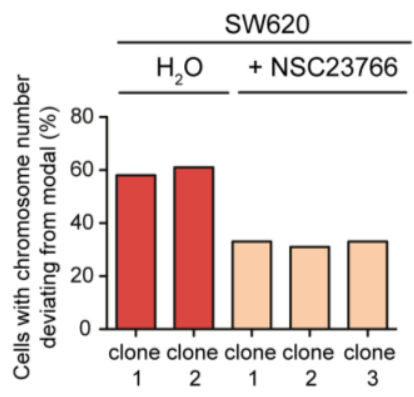

b

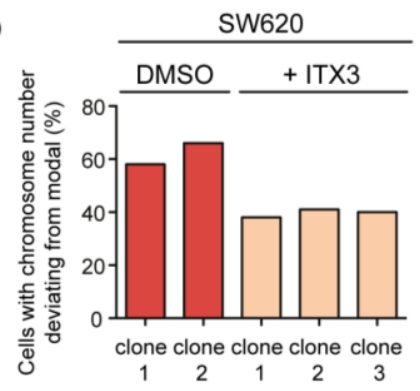

d

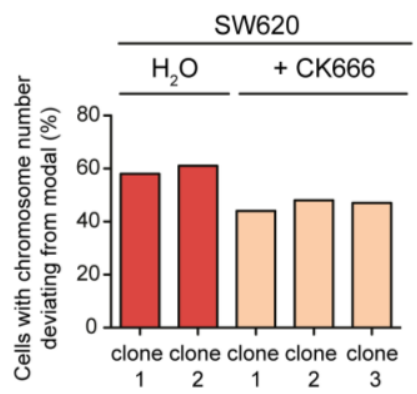

Fig. 3.21: CEP-FISH analyses of SW620 derived single cell clones treated with TRIO, Rac1 or Arp2/3 complex inhibitor. (a) CEP-FISH analyses detecting chromosome 7 and chromosome 15 in single cell clones derived from SW620 cells treated with DMSO or $0.2 \mathrm{nM}$ Taxol for 30 generations. The percentage of cells with a chromosome number deviating from modal was calculated $(n=100$ cells). (b) CEP-FISH analyses of single cell clones derived from SW620 cells treated with DMSO or $15 \mu \mathrm{M}$ ITX3 for 30 generations. The percentage of cells with a chromosome number deviating from modal was calculated ( $n=100$ cells). (c) CEP-FISH analyses of single cell clones derived from SW620 cells treated with $\mathrm{H}_{2} \mathrm{O}$ or $40 \mu \mathrm{M}$ NSC23766 for 30 generations. The percentage of cells with a chromosome number deviating from modal was calculated ( $n=100$ cells). (d) CEP-FISH analyses of single cell clones derived from SW620 cells treated with $\mathrm{H}_{2} \mathrm{O}$ or $20 \mu \mathrm{M}$ CK666 for 30 generations. The percentage of cells with a chromosome number deviating from modal was calculated $(n=100$ cells).

In order to verify the results obtained in SW620 cells, also SK-Mel-103 cells were analyzed after treatment with the different inhibitors by CEP-FISH analyses. Here, $\alpha$-satellite probes specific for chromosome 6 and chromosome 18 were used. Also in this cell line, the different treatments reduced the amount of cells with chromosome numbers deviating from modal. Treatment with Taxol, ITX3 and NSC23766 had a stronger effect than treatment with CK666. Whereas upon Taxol, ITX3 and NSC23766 treatment the amount of aneuploid cells was reduced by $18-24 \%$, CK666 treatment led to a reduction in aneuploid cells by $9-11 \%$ in the different single cell clones (Fig. 3.22a-d). 
a

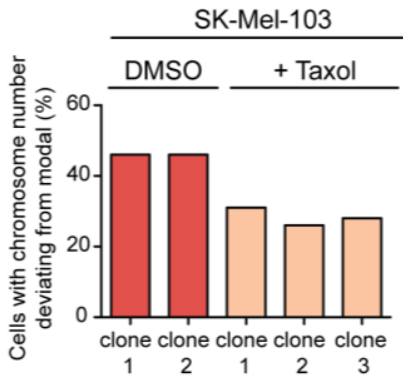

C

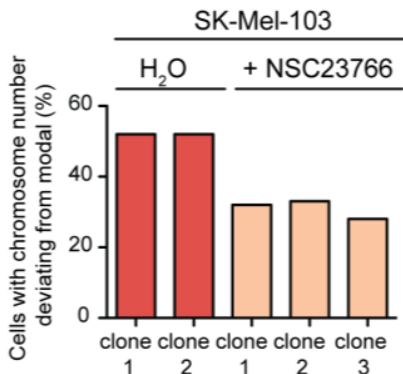

b

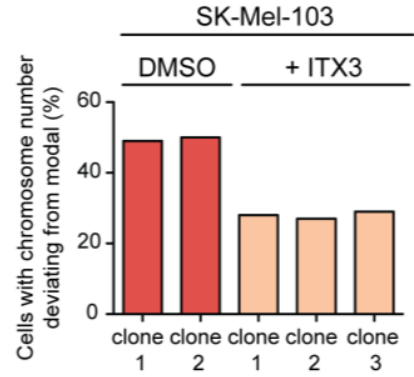

d

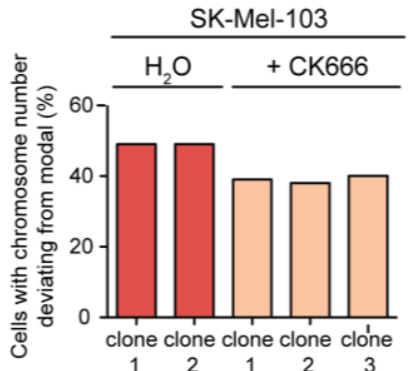

Figure 3.22: Inhibition of TRIO, Rac1 and Arp2/3 complex suppresses CIN in SK-Mel-103 cells. (a) CEP-FISH analyses detecting chromosome 6 and chromosome 18 in single cell clones derived from SK-Mel-103 cells treated with DMSO or $0.5 \mathrm{nM}$ Taxol for 30 generations. The percentage of cells with a chromosome number deviating from modal was calculated ( $n=100$ cells). (b) CEP-FISH analyses of single cell clones derived from SK-Mel-103 cells treated with DMSO or $15 \mu \mathrm{M}$ ITX3 for 30 generations. The percentage of cells with a chromosome number deviating from modal was calculated ( $n=100$ cells). (c) CEP-FISH analyses of single cell clones derived from SK-Mel-103 cells treated with $\mathrm{H}_{2} \mathrm{O}$ or $40 \mu \mathrm{M}$ NSC23766 for 30 generations. The percentage of cells with a chromosome number deviating from modal was calculated ( $n=100$ cells). (d) CEP-FISH analyses of single cell clones derived from SK-Mel-103 cells treated with $\mathrm{H}_{2} \mathrm{O}$ or $20 \mu \mathrm{M}$ CK666 for 30 generations. The percentage of cells with a chromosome number deviating from modal was calculated $(n=100$ cells).

In order to test whether long-term treatment of cells with the different inhibitors has an effect on cell migration and microtubule plus-end assembly rates, one single cell clone derived from Taxol, ITX3, NSC23766 and CK666 treated SW620 or SK-Mel-103 cells was analyzed. As expected, Taxol treatment reduced the microtubule plus-end assembly rates during mitosis and interphase in single cell clones (Fig. 3.23a). In contrast, treatment with ITX3, NSC23766 or CK666 did not alter microtubule plus-end assembly rates. Transwell migration assays were performed with a single cell clone derived from SK-Mel-103 cells treated with $0.5 \mathrm{nM}$ Taxol, $15 \mu \mathrm{M}$ ITX3, $40 \mu \mathrm{M}$ NSC23766 or $20 \mu \mathrm{M}$ CK666. Here, prolonged treatment with the different inhibitors reduced cell migration robustly (Fig. 3.23b). 
a

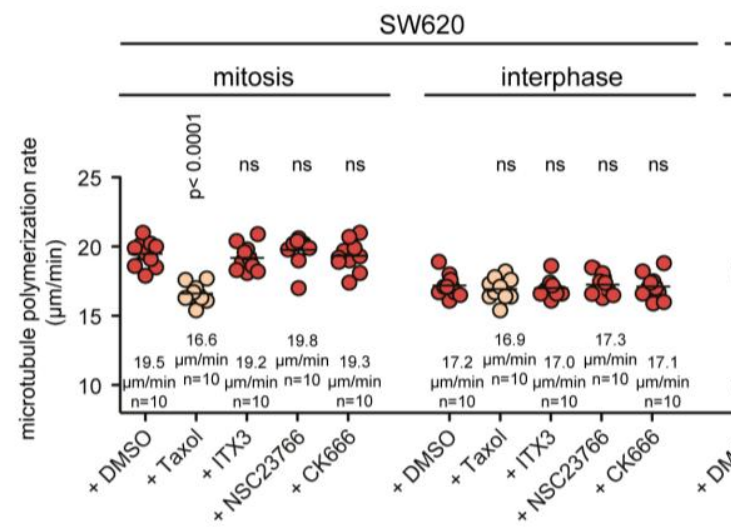

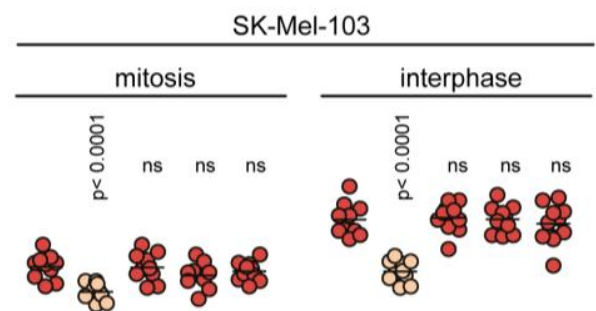

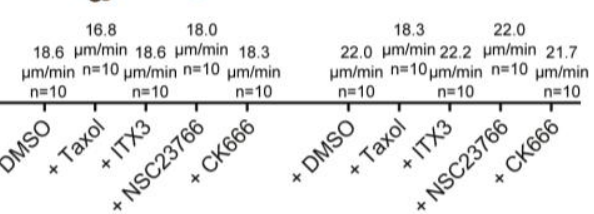

b cell migration

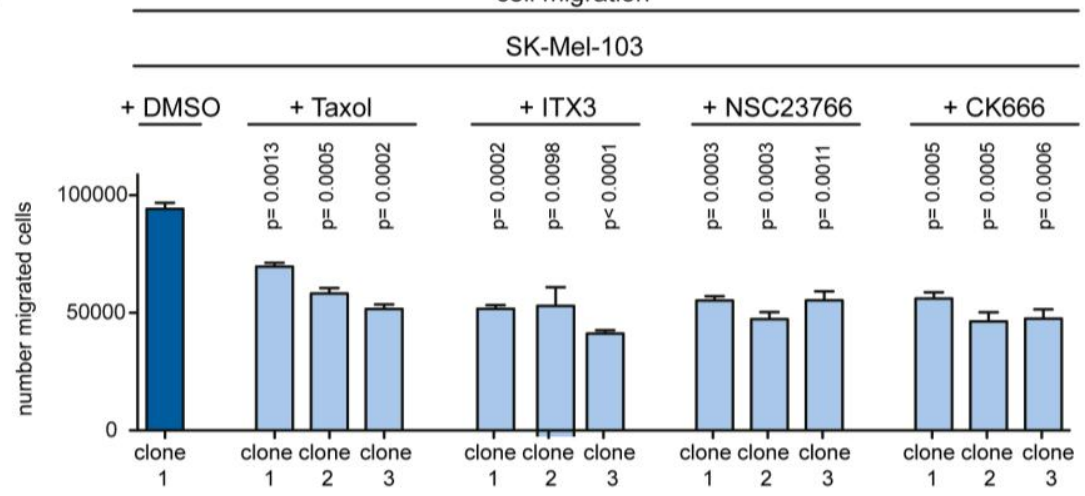

Figure 3.23: Single cell clones treated with inhibitor of TRIO, Rac1 or the Arp2/3 complex show no alterations in microtubule plus-end assembly rates but a reduce migratory phenotype. (a) Measurements of microtubule plus-end assembly rates during mitosis and interphase. Single cell clone 1 of SW620 (Fig. 3.20, Fig 3.21) and clone 1 of SK-Mel-103 (Fig. 3.22) generated over 30 generations in the presence of DMSO, $0.2 \mathrm{nM}$ (SW620) or $0.5 \mathrm{nM}$ (SK-Mel-103) Taxol, $15 \mu \mathrm{M}$ ITX3, $40 \mu \mathrm{M}$ NSC23766 and $20 \mu \mathrm{M}$ CK666 were treated with $2 \mu \mathrm{M}$ DME for $1 \mathrm{~h}-2 \mathrm{~h}$ prior to EB3 tracking experiments. Scatter dot plots show the average microtubule plus-end assembly rates of 20 microtubules per cell (mean \pm sem, $t$-test, $n=10$ cells). (b) Transwell migration assays of SK-Mel-103 single cell clones treated as described in (a). 200,000 cells were seeded in cell culture inserts. Bar graphs show the number of migrated cells after $24 \mathrm{~h}$ (mean \pm sem, $t$-test, $n=3$ ).

To further verify that the observed reduction in aneuploidy depends on the used drugs, the generated single cell clones were cultivated for further 30 generations in the absence of drugs. Afterwards, the chromosome numbers were determined by CEP-FISH analyses and compared to the chromosome copy numbers obtained before removal of the drug. These analyses revealed an increase in aneuploid cells in all SW620 and SK-Mel-103 cell clones after removal of the different drugs (Fig. 3.24a-b). Thus, CIN is re-introduced after reactivation of the TRIO-Rac1-Arp2/3 pathway. 
a

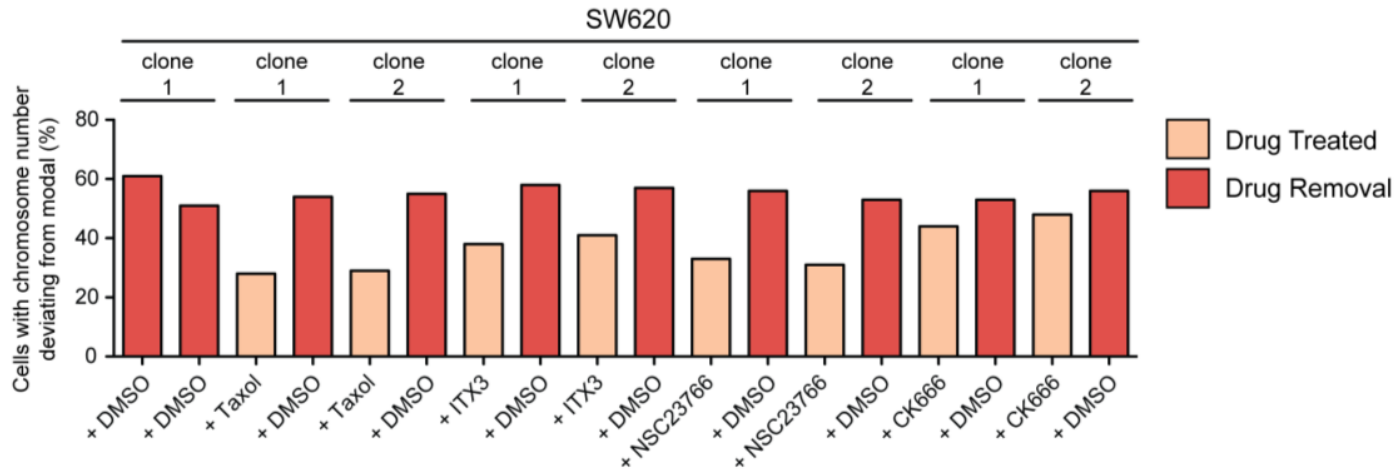

b

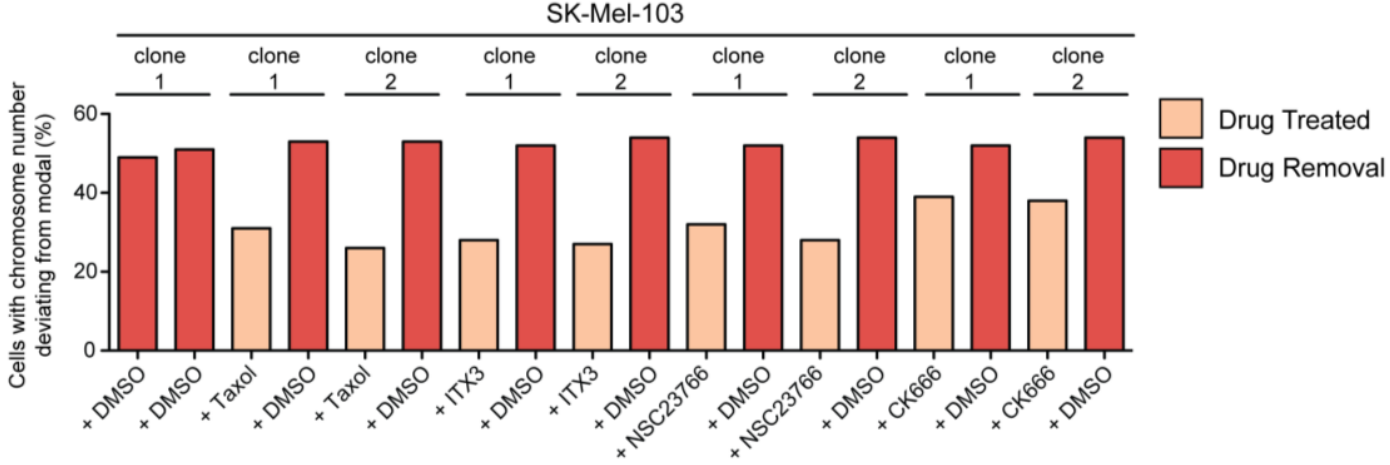

Figure 3.24: Drug removal re-induces CIN. (a) CEP-FISH analyses of SW620 cells before and after drug removal. After treatment with Taxol, ITX3, NSC23766 and CK666 for 30 generations, SW620 derived single cell clones were treated with DMSO for additional 30 generations. The percentage of cells with a chromosome number deviating from modal was determined ( $n=100$ cells). (b) CEP-FISH analyses of SK-Mel-103 cells before and after drug removal. After treatment with Taxol, ITX3, NSC23766 and CK666 for 30 generations, SK-Mel-103 derived single cell clones were treated with DMSO for additional 30 generations. The proportion of cells with a chromosome number deviating from modal was calculated ( $n=100$ cells). 


\subsection{The Role Of p53 And p73 In Chromosomal Instability And Migration}

\subsubsection{Loss Of TP53 And TP73 Increases Microtubule Plus-End Assembly Rates And Induces CIN}

A frequent lesion in the vast majority of human cancer types is a loss of function of the tumor suppressor p53. However, the loss of TP53 is not sufficient to induce aneuploidy in human cells (Bunz et al. 2002). The p53 homolog TP73 is located on chromosome arm 1p36, which is frequently deleted in various cancer types. This loss was shown to be associated with cancer (Kaghad et al. 1997). Furthermore, p73 suppresses polyploidy in the absence of p53 (Talos et al. 2007). Therefore, we were interested to investigate whether p53 and p73 might cooperate to suppress $\mathrm{CIN}$ in cancer cells.

To address this hypothesis, TP53 and TP73 were either separately or concomitantly repressed by siRNA in the chromosomal stable cell line HCT116 and microtubule plus-end assembly rates were measured. The knock down efficiency of TP53 and TP73 was confirmed by western blot (Fig. 3.25a).

a

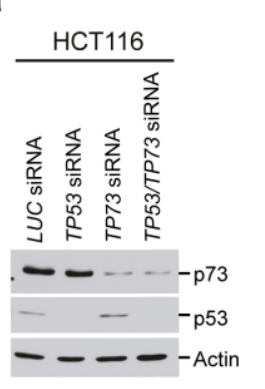

b

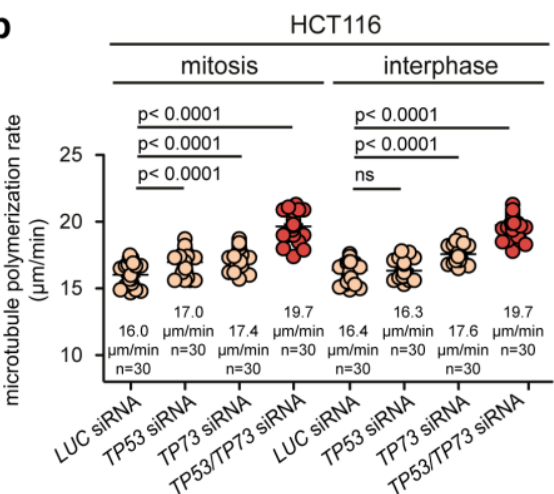

C

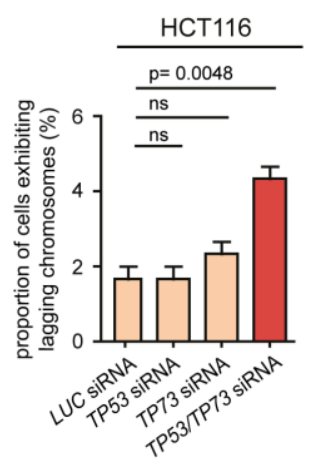

d

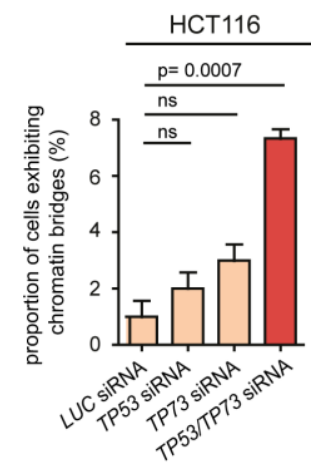

Figure 3.25: Concomitant repression of TP53 and TP73 leads to increased microtubule plusend assembly rates and induces the generation of lagging chromosomes. (a) Representative western blot showing the downregulation of TP53 and TP73 in response to siRNA transfection. $\beta$-actin was used as loading control. (b) Determination of microtubule plus-end assembly rates after repression of TP53, TP73 or both concomitantly. HCT116 cells were transfected with 60 pmol TP53, TP73 or LUCIFEARSE siRNA. For EB3 tracking experiments, cells were treated with $2 \mu \mathrm{M}$ DME for $1 \mathrm{~h}$. Scatter dot plots show the average microtubule plus-end assembly rates of 20 microtubules per cell (mean \pm sem, $t$-test, $n=30$ cells from three independent experiments). (c) Quantification of cells showing lagging chromosomes during anaphase in HCT116 cells after separate or concomitant repression of TP53 and TP73. Cells were synchronized at $\mathrm{G}_{1} / \mathrm{S}$ by double thymidine block and released into the cell cycle for $8.5 \mathrm{~h}$. Cells were fixed and stained for immunofluorescence microscopy. Bar graphs show the number of cells exhibiting at least one CREST-positive chromatid (mean \pm sem, $t$-test, $n=3$ with a total of 300 anaphase cells). (d) Quantification of cells exhibiting chromatin bridges. Cells were processed as described in (c). Bar graphs show the number of cells displaying chromatin bridges (mean \pm sem, $t$-test, $n=3$ with a total of 300 anaphase cells). 
The knock down of either TP53 or TP73 revealed only a slight increase in microtubule plusend assembly rates, whereas the simultaneous repression of both transcription factors greatly increased the microtubule plus-end assembly rates during mitosis and interphase when compared to LUCIFERASE siRNA transfected cells (Fig. 3.25b). During mitosis, the microtubule plus-end assembly rates were increased from $16.0 \mu \mathrm{m} / \mathrm{min}$ upon LUCIFERASE siRNA to $19.7 \mu \mathrm{m} / \mathrm{min}$ upon repression of both TP53 and TP73. During interphase, microtubule plus-end assembly rates were enhanced from $16.4 \mu \mathrm{m} / \mathrm{min}$ to $19.7 \mu \mathrm{m} / \mathrm{min}$.

Since increased microtubule plus-end assembly rates cause the generation of lagging chromosomes, the role of p53 and p73 during chromosome segregation was investigated. Cells were synchronized at $G_{1} / S$ transition by a double thymidine block and released into the cell cycle. Cells were fixed and stained after $8.5 \mathrm{~h}$ and cells in anaphase displaying lagging chromosomes were quantified. Importantly, the concomitant repression of TP53 and TP73 in chromosomal stable HCT116 cells induced the appearance of cells exhibiting lagging chromosomes from $1.6 \%$ to $4.3 \%$. The repression of either TP53 or TP73 alone did not significantly increase the proportion of cells with lagging chromosomes (Fig. 3.25c). Apart from the occurrence of lagging chromosomes, depletion of TP53 and TP73 led to highly increased numbers of cells showing chromatin bridges during anaphase (Fig. 3.25d).

Lagging chromosomes underlie CIN in cancer cells (Cimini et al. 2001). In order to further evaluate the impact of TP53 and TP73 on chromosomal stability, HCT116-TP53\% cells were used for the generation of stable single cell clones repressed of TP73 by using a TP73 shRNA expression plasmid. Furthermore, HCT116-TP53 ${ }^{-/}$cells were transfected with a control shRNA expression plasmid. HCT116-TP73 shRNA expression cells were available in our lab. TP73 repression was verified by western blot analyses (Fig. 3.26a). Stable cell clones with a significant repression of TP73 (TP73sh clones) were further analyzed regarding their microtubule plus-end assembly rates by EB3 tracking experiments. Here, cells with a repression of both transcription factors showed significantly increased microtubule plus-end assembly rates in mitosis and in interphase when compared to the single knock down cells HCT116-TP53 $\%$ and HCT116-TP73sh (Fig. 3.26b). The increased microtubule plus-end assembly rates also correlated with elevated amounts of cells with lagging chromosomes. Upon repression of both transcription factors, the amount of cells displaying lagging chromosomes was increased more than 3-fold compared to single knock down cells (Fig. 3.26c). Karyotype analyses of three individual single cell clones by individual chromosome counting in metaphase spreads revealed an increased amount of cells with chromosome numbers deviating from modal number of 45 after concomitant loss of TP53 and TP73 (Fig. 3.26d). The amount of aneuploid cells was increased from $12 \%$ in both control transfected HCT116-TP53 ${ }^{-/}$cell clones to $32 \%-44 \%$ in the three cell clones repressed of both transcription factors, indicating a chromosomal instability phenotype. 
a

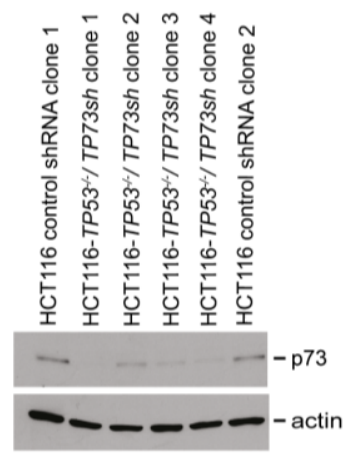

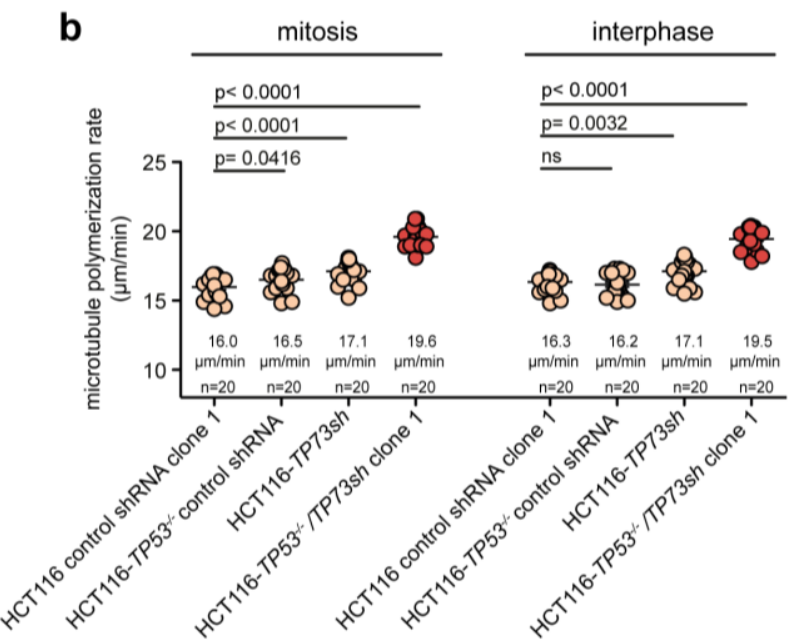

d

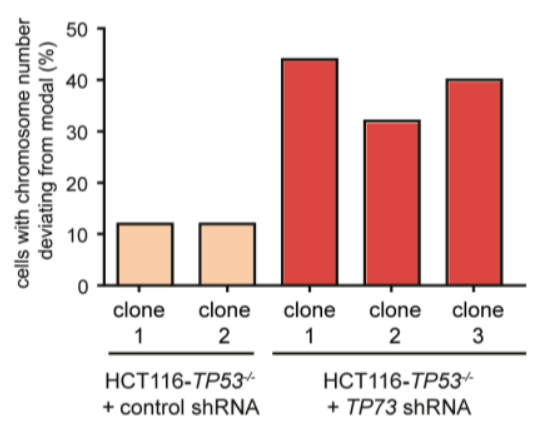

Figure 3.26: The concomitant repression of TP53 and TP73 leads to CIN. (a) HCT116-TP53-1 cells were stably transfected with a TP73 shRNA or a control shRNA expression plasmid and single cell clones were isolated. Knock down of TP73 was verified by western blot, whereby $\beta$-actin served as loading control. (b) EB3 tracking experiments of cells in mitosis and interphase. The indicated single cell clones were treated with $2 \mu \mathrm{M}$ DME $1 \mathrm{~h}$ prior to EB3 tracking experiments. Scatter dot plots show the average microtubule plus-end assembly rates of 20 microtubules per cell (mean \pm sem, $t$-test, $\mathrm{n}=20$ cells from two independent experiments). (c) Quantification of cells showing lagging chromosomes during anaphase. The indicated single cell clones were synchronized at $\mathrm{G}_{1} / \mathrm{S}$ transition by a double thymidine block and released into the cell cycle. Bar graphs show the proportion of cells exhibiting at least one CREST-positive chromatid (mean \pm sem, $t$-test, $\mathrm{n}=3$ with a total of 300 anaphase cells). (d) Induction of CIN after repression of TP53 and TP73. Karyotype analyses of single cell clones were performed by chromosome counting in metaphase spreads. The percentage of cells with a chromosome number deviating from modal was calculated ( $n=100$ cells).

These results showed that separate repression of TP53 or TP73 is not sufficient to induce chromosomal instability and aneuploidy in HCT116 cells. However, the concomitant repression of TP53 and TP73 induces CIN.

To test whether the occurrence of lagging chromosomes and CIN depends on the increased microtubule plus-end assembly rates (Fig. 3.25b), single cell clones repressed of TP53 and TP73 were treated with $0.2 \mathrm{nM}$ Taxol over 30 generations followed by drug removal for additional 30 generations. Upon low dose Taxol treatment, cells with low expression of TP53 
and TP73 showed decreased microtubule plus-end assembly rates, that were again increased after Taxol removal (Fig. 3.27a). Corresponding to that observation, the proportion of cells displaying lagging chromosomes increased after Taxol removal by about 3-4 fold (Fig. 3.27b). Karyotype analyses by counting individual chromosomes in metaphase spreads revealed between $20 \%$ and $25 \%$ of cells with a chromosome number deviating from modal upon Taxol treatment. After Taxol removal, the amount of aneuploid cells increased to $40 \%$ to $50 \%$ in the three different cell clones (Fig. 3.27c).

a
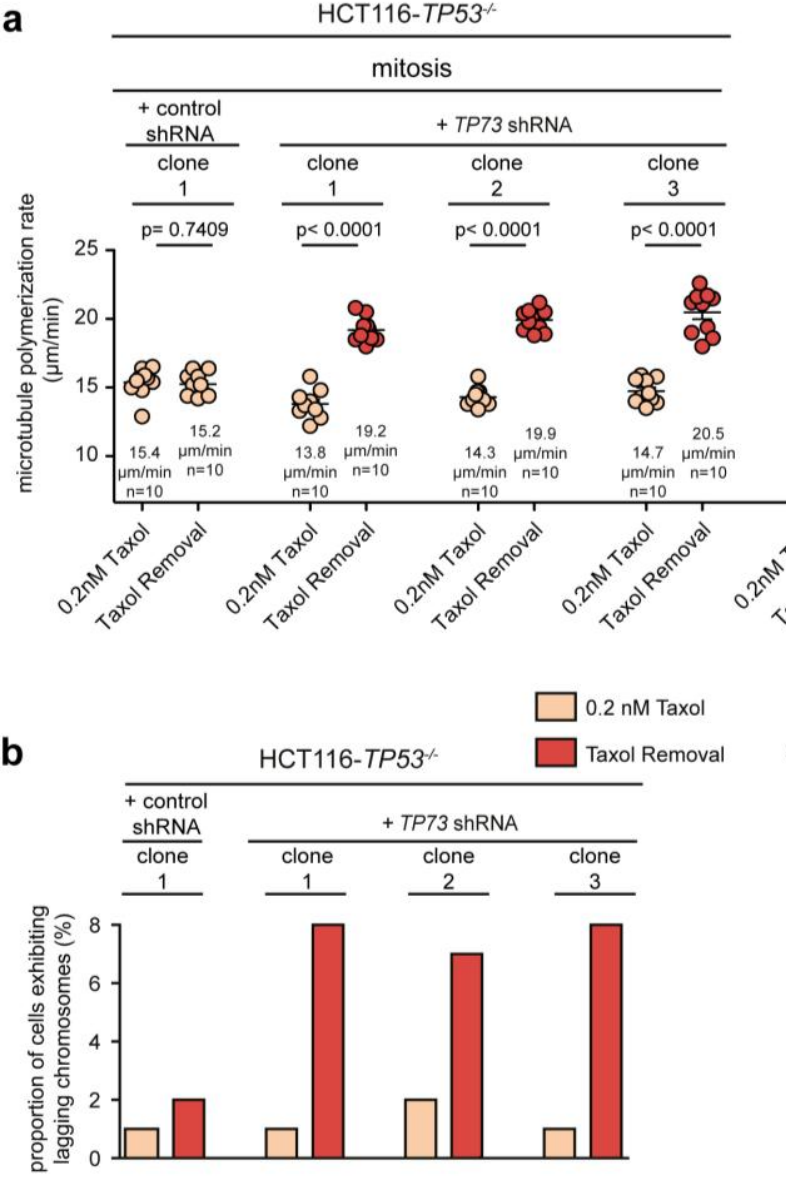

\begin{tabular}{|c|c|c|c|}
\hline \multicolumn{4}{|c|}{ НСТ116-TP53» } \\
\hline \multicolumn{4}{|c|}{ interphase } \\
\hline $\begin{array}{l}+ \text { control } \\
\text { shRNA }\end{array}$ & & + TP73 shRI & \\
\hline $\begin{array}{c}\text { clone } \\
1 \\
\end{array}$ & $\begin{array}{c}\text { clone } \\
1 \\
\end{array}$ & $\begin{array}{c}\text { clone } \\
2 \\
\end{array}$ & $\begin{array}{c}\text { clone } \\
3\end{array}$ \\
\hline$p=0.4207$ & $p<0.0001$ & $p<0.0001$ & $p<0.000$ \\
\hline
\end{tabular}
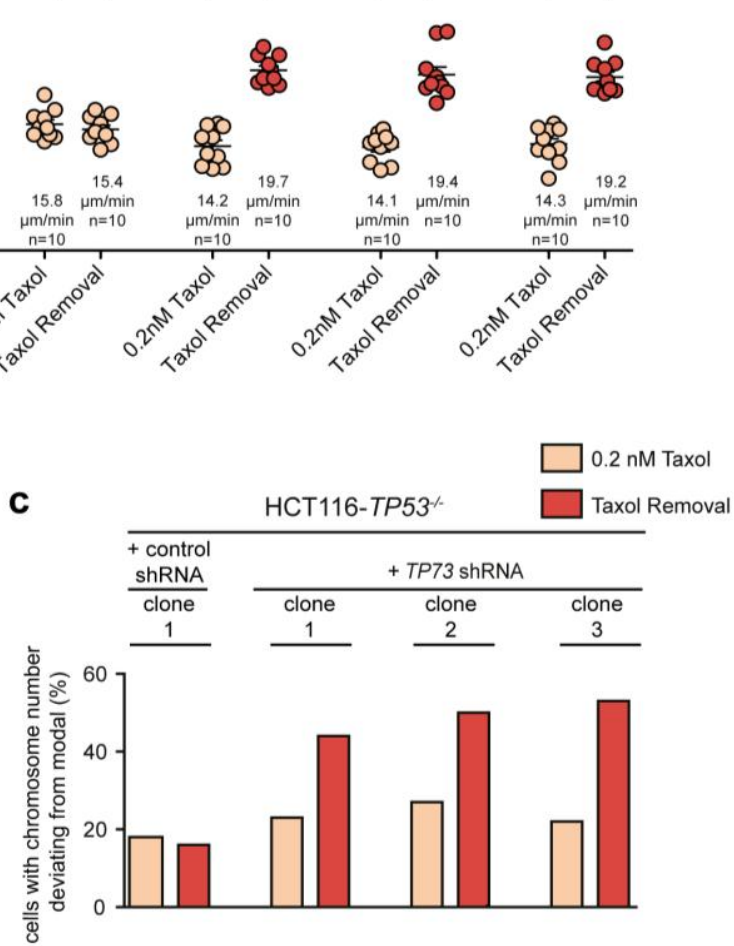

Figure 3.27: The CIN phenotype induced by loss of TP53 and TP73 in HCT116 cells can be suppressed by Taxol treatment. (a) Measurements of microtubule plus-end assembly rates in HCT116-TP53 ${ }^{-}$with a stable repression of TP73. Single cell clones were treated with $0.2 \mathrm{nM}$ Taxol for 30 generations, following removal of Taxol and cultivation in DMSO for additional 30 generations. Cells were treated with $2 \mu \mathrm{M}$ DME $1 \mathrm{~h}$ prior to EB3 tracking experiments. Scatter dot plots show the average microtubule plus-end assembly rates of 20 microtubules per cell (mean \pm sem, $t$-test, $n=10$ cells). (b) Quantification of the amount of cells exhibiting lagging chromosomes during anaphase. Single cell clones were generated as described in (a). Cells were synchronized at $\mathrm{G}_{1} / \mathrm{S}$ transition by double thymidine block, released into the cell cycle for $8.5 \mathrm{~h}$ and fixed and stained for immunofluorescence microscopy. Bar graphs show the proportion of cells displaying at least one CREST-positive chromatid ( $n=100$ anaphase cells counted). (c) Karyotype analyses of single cell clones. Single cell clones were generated as described in (a). The proportion of cells with a chromosome number deviating from modal was calculated ( $n=100$ cells). 
These results indicate a causal role for increased microtubule plus-end assembly rates induced by loss of p53 and p73 for the development of CIN.

To exclude unspecific effects of the TP73 shRNA construct, TAp73 was re-expressed in HCT116-TP53 $/$ TP73sh cells. Furthermore, TAp73 was re-expressed in HCT116 and HCT116-TP53 ${ }^{-1}$ cells, which served as control cell lines. Stable cell clones were generated by the use of a doxycycline inducible TAp73 expression vector. The re-expression of TAp73 in individual single cell clones was confirmed by western blot analyses (Fig. 3.28a).

a
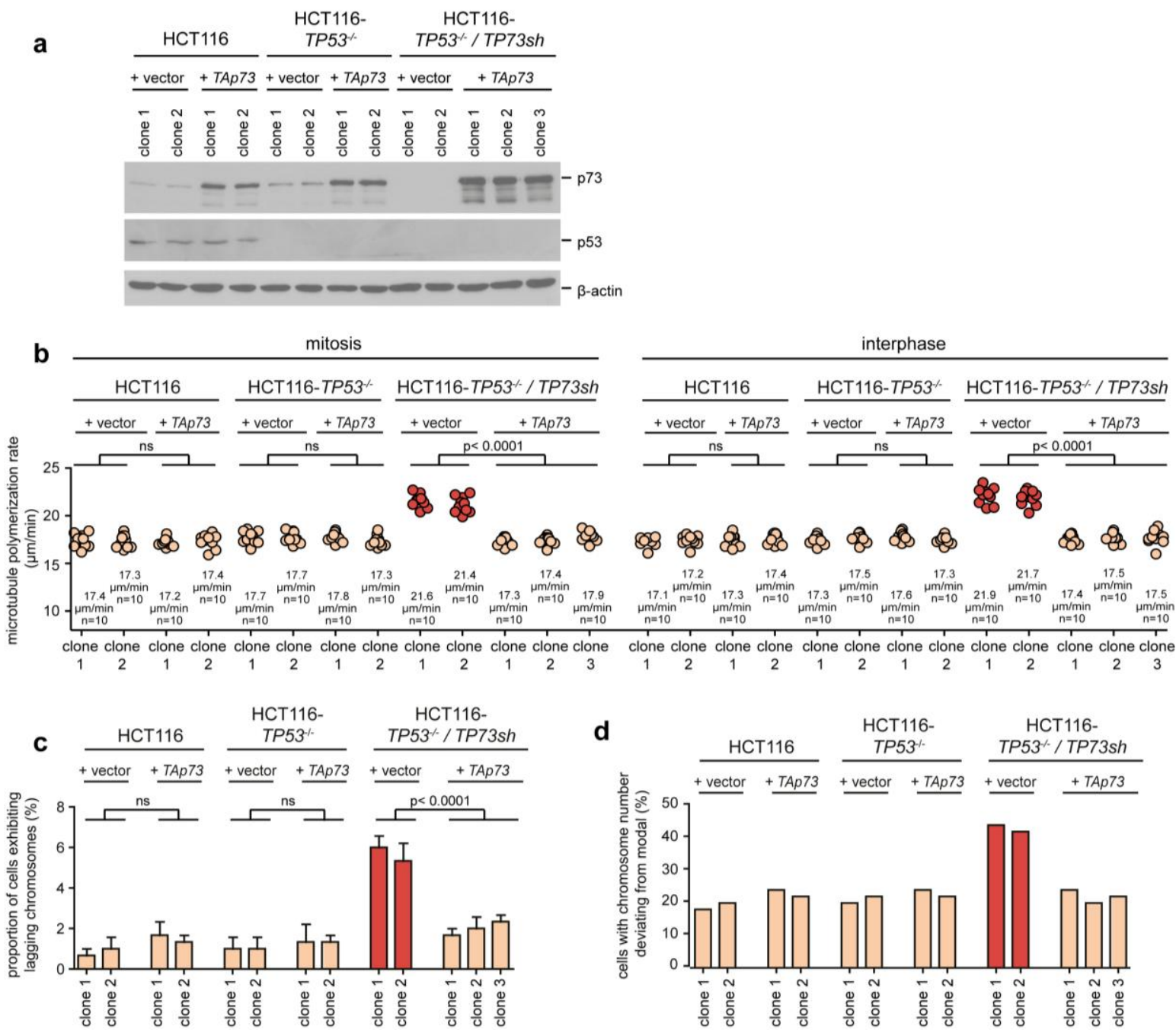

Figure 3.28: The re-expression of TAp73 suppresses CIN in HCT116-TP53-/TP73sh cells. (a) TAp73 was stably re-expressed in HCT116, HCT116-TP53 $3^{-/}$and HCT116-TP53 $3^{-/}$/TP73sh cells and single cell clones were isolated. The re-expression of TAp73 was verified by western blot. $\beta$-actin served as a loading control (b) Measurements of microtubule plus-end assembly rates in single cell clones, that are shown in (a). Cells were treated with $2 \mu \mathrm{M}$ DME $1 \mathrm{~h}$ prior to EB3 tracking experiments. Scatter dot plots show the average microtubule plus-end assembly rates of 20 microtubules per cell (mean \pm sem, $t$-test, $n=10$ cells). (c) Quantification of the proportion of cells showing lagging chromosomes in single cell clones, that are shown in (a). Cells were synchronized at $\mathrm{G}_{1} / \mathrm{S}$ transition by double thymidine block, released into the cell cycle and fixed and stained for immunofluorescence microscopy after $8.5 \mathrm{~h}$. Bar graphs show the quantification of cells exhibiting at least one CREST-positive chromatid (mean \pm sem, $t$-test, $n=3$ with a total of 300 anaphase cells). (d) Karyotype analyses of the indicated single cell clones. The proportion of cells with a chromosome number deviating from modal was determined by chromosome counting in metaphase spreads $(n=100$ cells). 
EB3 tracking experiments were performed to investigate the microtubule plus-end assembly rates. The control cells (HCT116-TAp73 and HCT116-TP53 ${ }^{-/}$-TAp73) were not altered in their microtubule plus-end assembly rates, revealing that overexpression of TAp73 does not affect microtubule dynamics. However, re-expression of TAp73 in HCT116-TP53 ${ }^{-1} /$ TP73sh cells decreased the microtubule plus-end assembly rates during interphase and mitosis comparable to control transfected HCT116-TP53//TP73sh cells (Fig. 3.28b). Consequently, the amount of cells showing lagging chromosomes was decreased approximately 3 -fold upon re-expression of TAp73 in HCT116-TP53//TP73sh cells, whereas the generation of lagging chromosomes was not affected in HCT116 and HCT116-TP53 ${ }^{-/}$cells (Fig. 3.28c). Karyotype analyses by chromosome counting from metaphase spreads revealed a decreased amount of cells with a chromosome number deviating from modal in HCT116-TP53/ $/$ TP73sh cells exhibiting a stable re-expression of TAp73 compared to control transfected HCT116-TP53 $3^{-/} /$PP73sh cells (Fig. 3.28d). These results support the hypothesis that $\mathrm{p} 53$ and p73 cooperate in maintaining both, proper microtubule plus-end assembly rates in mitosis and chromosomal stability.

So far, cells with a knock out of TP53 were used. However, this situation is not a common feature of cancer cells. Instead, human cancer cells rather exhibit a loss of function of p53 due to mutations. Different hot spot mutations were described including a most frequent p53 R175H mutation (Muller \& Vousden 2013). HCT116 cells expressing a TP53 R175H mutant where used to further evaluate the importance of both p53's and p73's function for the maintenance of chromosomal stability. HCT116-p53-R175H single cell clones stably transfected with control shRNA or TP73 shRNA expression plasmids were isolated and the knock down of TP73 was verified by western blot analyses (Fig. 3.29a). Subsequently, microtubule plus-end assembly rates were determined. While the p53 mutation alone did not affect microtubule plus-end assembly rates, the stable repression of TP73 in HCT116-p53$\mathrm{R} 175 \mathrm{H}$ cells resulted in an increase in microtubule plus-end assembly rates. Furthermore, the cell clones were treated with $0.2 \mathrm{nM}$ Taxol $2 \mathrm{~h}$ prior to EB3 tracking experiments. The low dose Taxol treatment led to a decrease in microtubule plus-end assembly rates to levels similarly observed in the control transfected HCT116-p53-R175H cell clone 1 (Fig. 3.29b). Analyses of HCT116-p53-R175H cells with low TP73 expression revealed an increased proportion of cells displaying lagging chromosomes in comparison to control transfected HCT116-p53-R175H cells. While the proportion of control cells exhibiting lagging chromosomes was in a range from $1 \%-2 \%$, on average $5 \%-5.6 \%$ of the single cell clones derived from HCT116-p53-R175H repressed of TP73 showed lagging chromosomes (Fig. 3.29c). Subsequently, karyotype analyses by chromosome counting from metaphase spreads were conducted. Importantly, HCT116-p53-R175H cell clones with low TP73 expression also displayed a higher amount of cells with a chromosome number deviating 
from modal. This number was increased from $20 \%-26 \%$ in control transfected HCT116-p53$\mathrm{R} 175 \mathrm{H}$ cells to $43 \%-46 \%$ in the three HCT116-p53-R175H cell clones repressed of TP73 (Fig. 3.29d), indicating that the TP53 mutation in combination with loss of TP73 induced CIN.

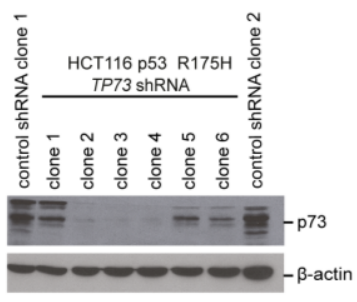

b

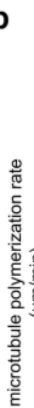

HCT116 p53 R175H

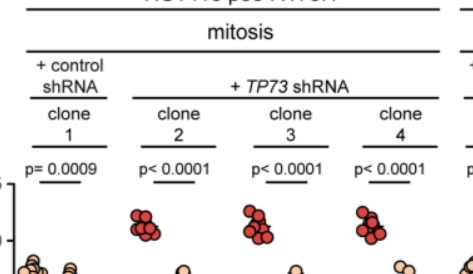

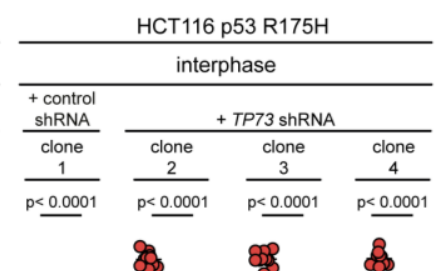

(8)
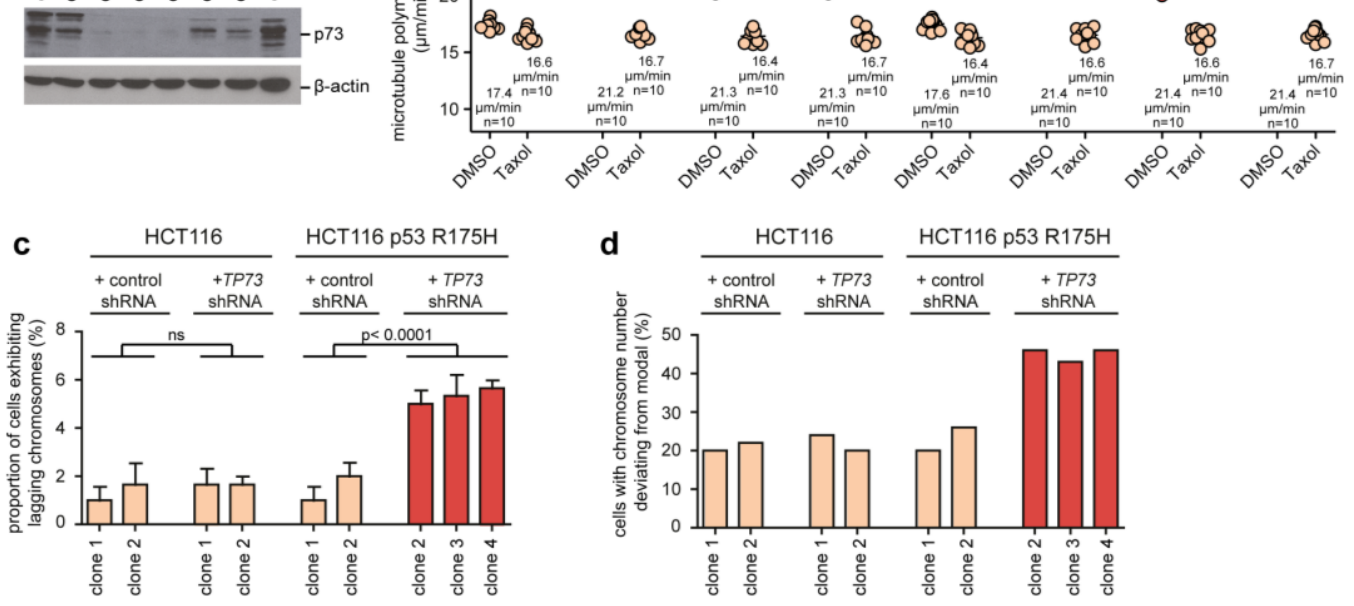

Figure 3.29: Repression of TP73 in HCT116 cells expressing a mutant form of p53 induces CIN. (a) TP73 was stably repressed in HCT116 cells expressing mutant TP53 (p53-R175H) by shRNA and single cell clones were generated. The repression of TP73 was verified by western blot. $\beta$-actin served as loading control. (b) Measurements of microtubule plus-end assembly rates in the single cell clones shown in (a). Cells were treated with $2 \mu \mathrm{M}$ DME $1 \mathrm{~h}$ prior to EB3 tracking. Scatter dot plots show the average microtubule plus-end assembly rates of 20 microtubules per cell (mean \pm sem, $t$-test, $\mathrm{n}=10$ cells). (c) Quantification of proportion of cells with lagging chromosomes in the single cell clones shown in (a). Cells were synchronized by double thymidine block, released into the cell cycle and fixed for immunofluorescence microscopy after $8.5 \mathrm{~h}$. Bar graphs show quantification of cells exhibiting at least one CREST-positive chromatid (mean \pm sem, $t$-test, $n=3$ with a total of 300 anaphase cells). (d) Karyotype analyses of the indicated single cell clones by individual chromosome counting from metaphase spreads. The percentage of cells with a chromosome number deviating from modal was determined ( $n=100$ cells).

Another common genetic alteration of cancer cells is the overexpression of a truncated form of $\mathrm{p} 73$, which is $\Delta N p 73 . \Delta \mathrm{Np} 73$ is described to inhibit both, p53's and p73's function and might act in a dominant negative manner (Zaika et al. 2002). To investigate the influence of $\Delta N p 73$ expression on microtubule plus-end assembly rates, $\Delta N p 73$ was overexpressed in HCT116 cells. To verify the inhibition of p53's function by $\Delta N p 73$, cells were treated with $600 \mathrm{nM}$ adriamycin for $16 \mathrm{~h}$ to induce DNA damage and the activation of p53. Western blot analyses were performed and the protein level of the p53 target p21 was examined. While the overexpression of $\Delta N p 73$ did not alter the protein level of p53 itself, the p53-mediated induction of p21 was greatly decreased suggesting that p53 function is inhibited (Fig. 3.30a). 
Furthermore, EB3 tracking experiments were performed. The overexpression of $\triangle N p 73$ in HCT116 cells also increased the microtubule plus-end assembly rates during both, mitosis and interphase in comparison to control transfected cells (Fig. 3.30b). Moreover, in the same cells, the proportion of cells with lagging chromosomes was also increased (Fig. 3.30c).
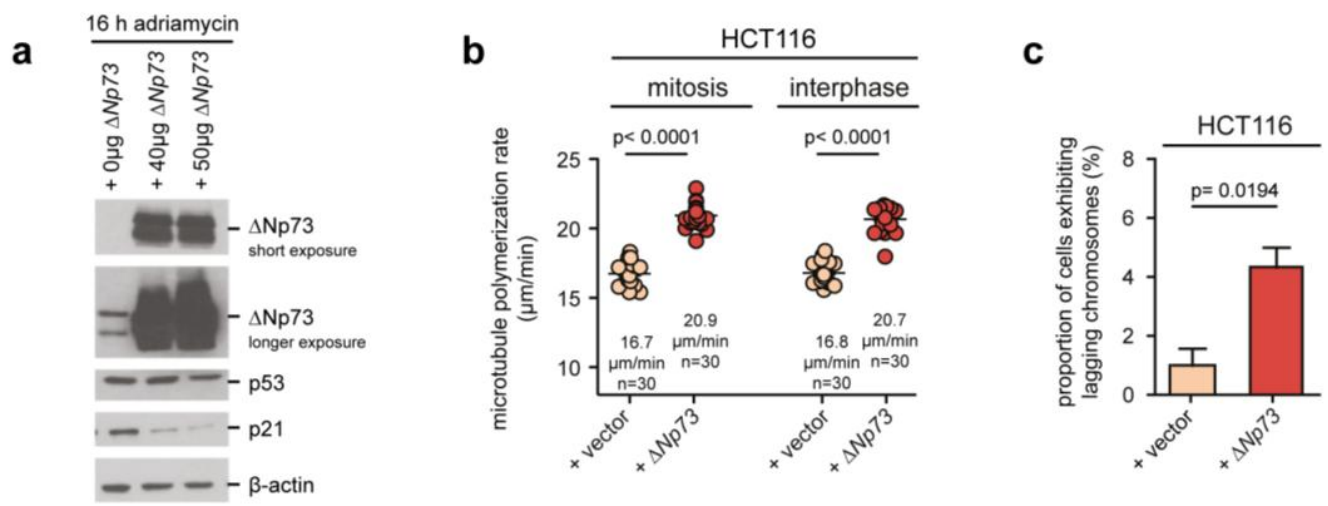

Figure 3.30: The expression of $\Delta N p 73$ increases microtubule plus-end assembly rates and induces the occurrence of lagging chromosomes. (a) $\triangle N p 73$ was transiently overexpressed in HCT116 cells. Cells were treated with $600 \mathrm{nM}$ adriamycin for $16 \mathrm{~h}$ and p21 protein levels were detected on western blot. $\beta$-actin was used as a loading control. (b) Measurements of microtubule plus-end assembly rates after overexpression of $\Delta N p 73$. Cells were treated with $2 \mu \mathrm{M}$ DME $1 \mathrm{~h}$ prior to EB3 tracking experiments. Scatter dot plots show the average microtubule plus-end assembly rates of 20 microtubules per cell (mean \pm sem, $t$-test, $n=30$ cells from three independent experiments). (c) Quantification of cells with lagging chromosomes during anaphase in HCT116 cells after transient overexpression of $\Delta N p 73$. Cell cycle synchronization at $\mathrm{G}_{1} / \mathrm{S}$ transition was achieved by a double thymidine block. Cells were released into the cell cycle for $8.5 \mathrm{~h}$ and fixed and stained for immunofluorescence microscopy. Bar graphs show the proportion of cells displaying at least one CREST-positive chromatid (mean \pm sem, $t$-test, $n=3$ with a total of 300 anaphase cells).

These results indicate that expression of $\Delta N p 73$ is sufficient to functionally inactivate both, p53 and p73 and to mediate an increase of microtubule plus-end assembly rates and chromosome segregation defects.

\subsubsection{Abnormal Microtubule Dynamics Induced By Loss Of TP53 And TP73 Are Mediated By p21}

Since p53 and p73 are transcription factors it is likely that upon loss of both various genes might not be transactivated. In collaboration with the Transcriptome and Genome Analysis Laboratory (TAL) of the University Göttingen RNA sequencing of HCT116 cells synchronized in $\mathrm{G}_{2}$ was performed. For this, HCT116 cells were separately or concomitantly depleted of TP53 and TP73by siRNA. Analyses of the data were performed by Dr. Silvia von der Heyde (former University of Göttingen, Göttingen, Germany). The following table shows an extract of genes, which were deregulated upon separate or concomitant loss of TP53 and TP73. 
Table 3.1: Extract of deregulated genes after single or concomitant loss of TP53 and TP73. FC: fold change

\begin{tabular}{|lc|lc|lc|}
\hline \multicolumn{2}{|c|}{$\begin{array}{c}\text { TP53 siRNA } \\
\text { vs } \\
\text { LUC siRNA }\end{array}$} & \multicolumn{2}{c|}{$\begin{array}{c}\text { TP73 siRNA } \\
\text { vs } \\
\text { LUC siRNA }\end{array}$} & \multicolumn{2}{c|}{$\begin{array}{c}\text { TP53/TP73 siRNA } \\
\text { vs }\end{array}$} \\
\hline \hline gene & FC & gene & FC & gene & FC \\
\hline TP53 & 0.21 & TP73 & 0.46 & TP53 & 0.22 \\
CDKN1A & 0.39 & CDKN1A & 0.92 & TP73 & 0.43 \\
TP53INP1 & 0.28 & RMRP & 0.27 & CDKN1A & 0.31 \\
MDM2 & 0.50 & RPPH1 & 0.34 & TP53INP1 & 0.31 \\
BAX & 0.65 & GATA3 & 0.46 & MDM2 & 0.48 \\
SESN1 & 0.46 & SNORA21 & 0.48 & BAX & 0.60 \\
DNALI1 & 2.21 & AKAP5 & 2.00 & IQGAP2 & 2.02 \\
CDH4 & 2.26 & SH2D3C & 2.03 & TGFBI & 3.96 \\
\hline
\end{tabular}

Among the deregulated genes, the CDK-inhibitor p21 (encoded by the CDKN1A gene) was found, which is a key target gene of p53 (El-Deiry et al. 1993). To verify the RNA sequencing results, qRT-PCR analyses were performed to investigate the relative CDKN1A mRNA levels in cells repressed of either TP53, TP73 or both concomitantly. The analyses verified low expression of CDKN1A in HCT116-TP53 ${ }^{-\alpha}$ cells compared to parental HCT116 cells. Repression of TP73 alone also decreased CDKN1A mRNA level, but not as strong as observed upon repression of TP53. In cells repressed of both TP53 and TP73, the CDKN1A mRNA levels were even more decreased compared to HCT116-TP53\% cells (Fig. 3.31a).

a

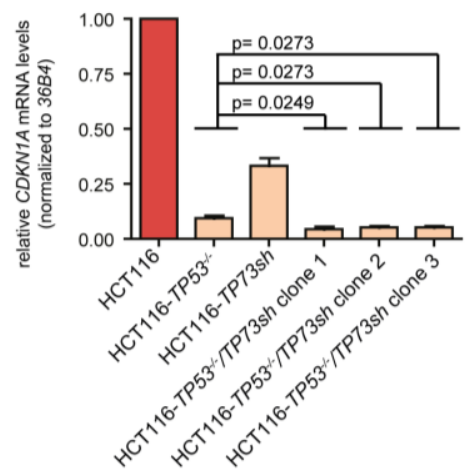

b

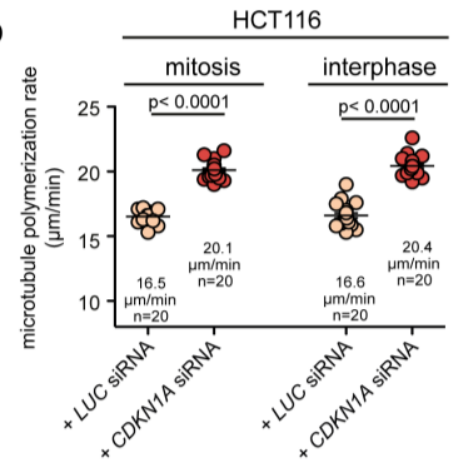

C

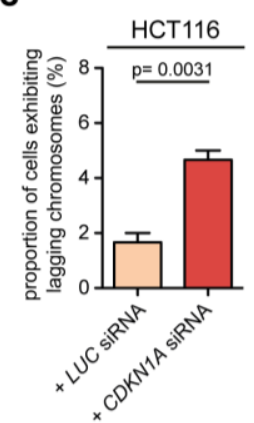

Figure 3.31: Repression of CDKN1A induces increased microtubule plus-end assembly rates and lagging chromosomes. (a) qRT-PCR analyses to quantify CDKN1A expression in cells repressed of TP53 and TP73. RNA was extracted from asynchronously growing cells and cDNA was synthesized. qRT-PCR was performed with gene specific primers. CDKN1A mRNA levels were normalized to the housekeeping gene 36B4. Bar graphs show relative mRNA expression levels (mean \pm sem, $t$-test, $n=3$ ) (b) Measurements of microtubule plus-end assembly rates in HCT116 cells after transfection with $60 \mathrm{pmol}$ LUCIFERASE or CDKN1A siRNA. Scatter dot plots show the average microtubule plus-end assembly rates of 20 microtubules per cell (mean \pm sem, $t$-test, $n=20$ cells from two independent experiments). (c) Quantification of cells with lagging chromosomes after siRNAmediated repression of CDKN1A in HCT116 cells. Cells were synchronized at $\mathrm{G}_{1} / \mathrm{S}$ transition by double thymidine block and released into the cell cycle. After $8.5 \mathrm{~h}$ cells were fixed and stained for immunofluorescence microscopy. Bar graphs show proportion of cells exhibiting at least one CRESTpositive chromatid (mean \pm sem, $t$-test, $\mathrm{n}=3$ with a total of 300 anaphase cells). 
Subsequently, CDKN1A was repressed in HCT116 cells by siRNA. EB3 tracking experiments showed significantly increased microtubule plus-end assembly rates upon CDKN1A repression compared to LUCIFERASE siRNA transfected cells (Fig. 3.31b). Furthermore, the occurrence of lagging chromosomes was analyzed. While LUCIFERASE siRNA transfected cells exhibited $1.6 \%$ lagging chromosomes, this proportion was increased to $4.6 \%$ on average in CDKN1A siRNA transfected cells (Fig. 3.31c).

To elucidate the relevance of p21 for increased microtubule plus-end assembly rates in HCT116 cells induced by concomitant repression of TP53 and TP73, CDKN1A was re-expressed in these cells. The overexpression of $C D K N 1 A$ was verified by western blot analyses (Fig. 3.32a).

a

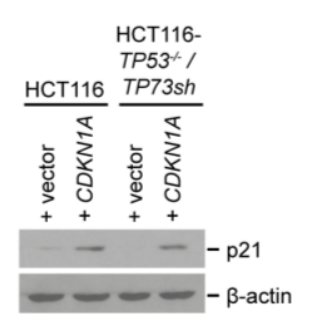

C

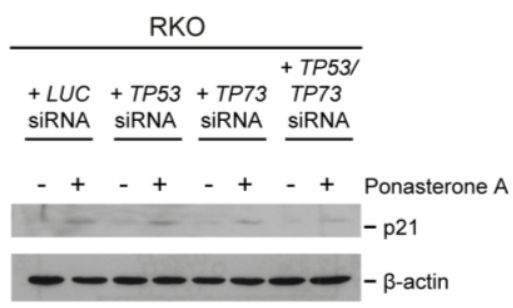

b

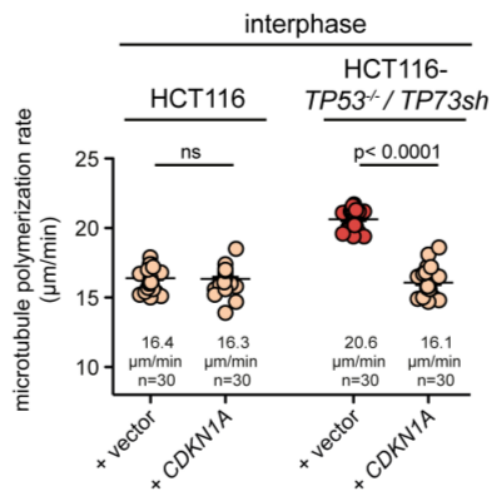

d

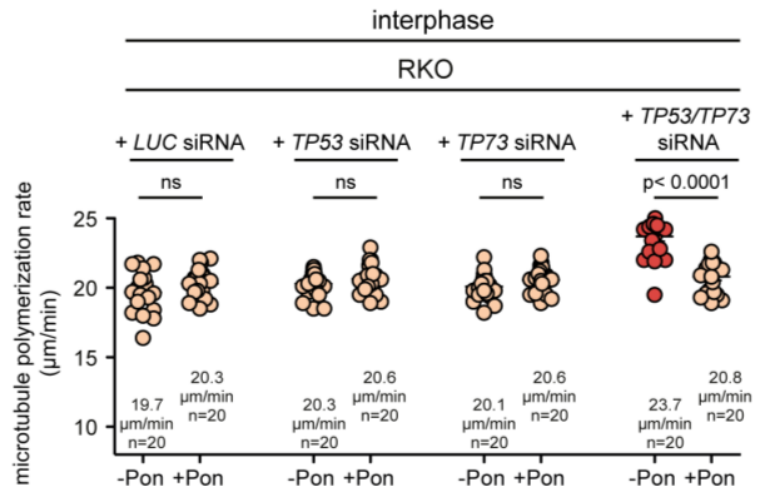

Figure 3.32: The re-expression of CDKN1A restores increased microtubule plus-end assembly rates observed upon repression of TP53 and TP73 in HCT116 and RKO cells. (a) Representative western blot verifying CDKN1A expression in HCT116 cells. $\beta$-actin was used as loading control. (b) Measurements of microtubule plus-end assembly rates in HCT116 and HCT116-TP53//TP73shRNA upon re-expression of CDKN1A. Scatter dot plots show the average microtubule plus-end assembly rates of 20 microtubules per cell (mean \pm sem, $t$-test, $n=30$ cells from three independent experiments). (c) Representative western blot showing CDKN1A induction upon ponasterone $A$ treatment in RKO cells. $\beta$-actin was used as loading control. (d) Measurements of interphase microtubule plus-end assembly rates in RKO cells, expressing CDKN1A upon ponasterone A (Pon) treatment. Cells were transfected with $60 \mathrm{pmol}$ siRNA and treated with $5 \mu \mathrm{mol} / \mathrm{l}$ ponasterone $A$ for CDKN1A induction. Scatter dot plots show the average microtubule plus-end assembly rates of 20 microtubules per cell (mean \pm sem, $t$-test, $n=20$ cells from two independent experiments). 
Subsequently, the microtubule plus-end assembly rates were examined. Since the overexpression of $C D K N 1 A$ led to a cell cycle arrest in $\mathrm{G}_{1}$-phase, the microtubule plus-end assembly rates could solely be measured in interphase cells. The re-expression of CDKN1A resulted in a decrease in microtubule plus-end assembly rates in comparison to control vector transfected HCT116-TP53//TP73sh cells (Fig. 3.32b). The microtubule plus-end assembly rates measured upon $C D K N 1 A$ re-expression were comparable to those measured in control HCT116 cells.

To further strengthen these findings, RKO cells harboring a ponasterone $A$ inducible CDKN1A expression plasmid were used (Schmidt et al. 2000). Also here, the induction of CDKN1A via ponasterone $A$ treatment arrested the cells in $\mathrm{G}_{1}$-phase and microtubule plusend assembly rates were only measured in interphase. Cells were repressed of either TP53 or TP73 or both concomitantly by siRNA transfection and the ponasterone A inducible $C D K N 1 A$ expression plasmid was overexpressed. Treatment with $5 \mu \mathrm{mol} / /$ ponasterone $A$ led to the induction of CDKN1A expression, which was verified by western blot analyses (Fig. 3.32c). EB3 tracking experiments revealed, that also in RKO cells, the loss of both TP53 and TP73 resulted in an increase in microtubule plus-end assembly rates during interphase, which was reduced upon re-expression of CDKN1A (Fig. 3.32d). Therefore, it is possible that p21 acts as an important effector of both, p53 and p73, for regulating microtubule plus-end assembly rates in these cells.

\subsubsection{Loss of TP53 And TP73 Causes An Invasive Phenotype In HCT116 Cells}

Interestingly, loss of TP53 and TP73 induced not only increased microtubule plus-end assembly rates during mitosis but also in interphase. As shown in the first part of this thesis, increased microtubule plus-end assembly rates in interphase are associated with increased cell migration and invasion. Therefore, transwell invasion assays for cells with loss of TP53 and TP73 were performed. HCT116, HCT116-TP53 ${ }^{-/}$, HCT116-TP73shRNA cells and three individual cell clones of HCT116-TP53 ${ }^{-\alpha}$ cells with a stable repression of TP73 were used. Furthermore, one HCT116 TP53 $3^{-/}$TP73sh cell clone, which was generated in the presence of low dose Taxol (Fig. 3.27), was analyzed to examine the influence of microtubule plus-end assembly rates on invasion (Fig. 3.33a). Transwell invasion assays revealed a significantly increased number of invasive cells after concomitant repression of TP53 and TP73 in HCT116 cells (Fig. 3.33a). The repression of either TP53 or TP73 alone did not alter the invasion potential in comparison to control HCT116 cells. Importantly, low dose Taxol treatment reduced the amount of invading cells to levels comparable to parental HCT116 cells, suggesting that also in cells with loss of TP53 and TP73 the invasive phenotype is mediated by increased microtubule plus-end assembly rates. 

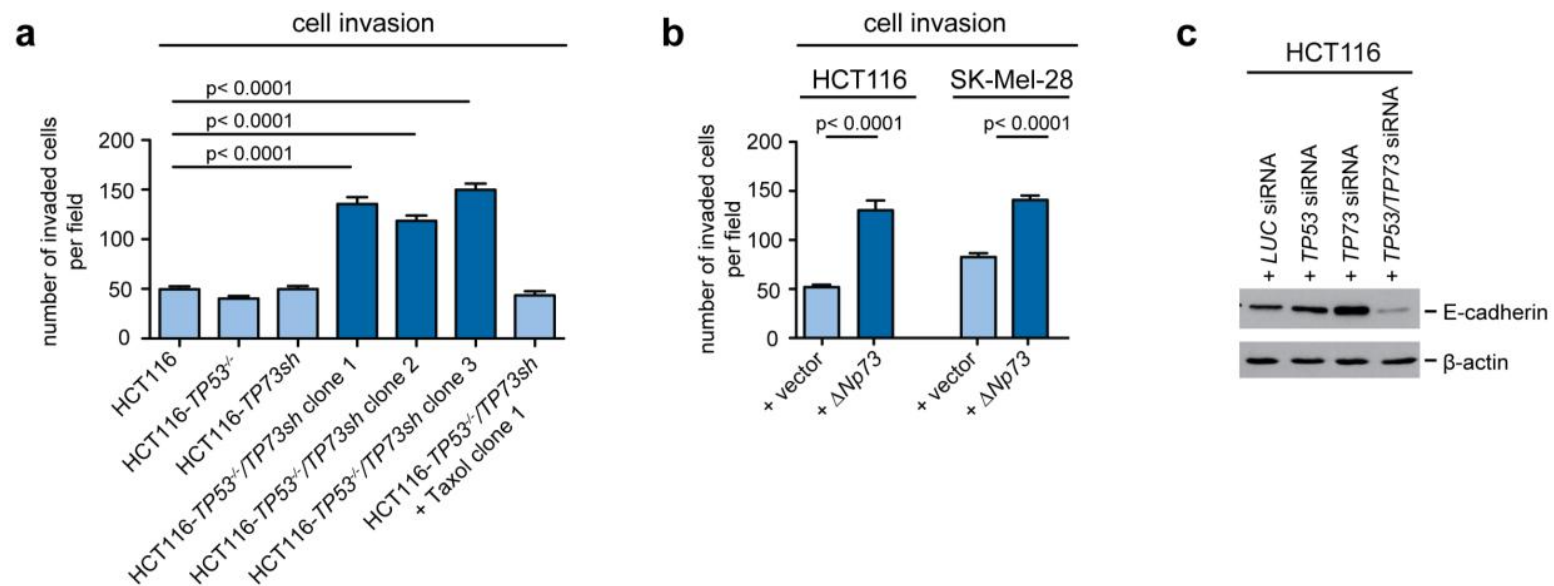

Figure 3.33: Loss of TP53 and TP73 induces invasion. (a) Transwell invasion assays using HCT116, HCT116-TP53 ${ }^{-/}$, HCT116-TP73shRNA cells, three individual cell clones of HCT116-TP53 ${ }^{-1}$ cells with a stable repression of TP73 and one Taxol treated cell clone. Bar graphs show the amount of invaded cells in 30 randomly chosen pictures from three independent experiments ( $t$-test, mean \pm sem, $n=3$ ). (b) Transwell invasion assays of HCT116 and SK-Mel-28 cells after overexpression of $50 \mu \mathrm{g} \Delta \mathrm{Np} 73$. Bar graphs show the amount of invaded cells in 30 randomly chosen pictures from three independent experiments ( $t$-test, mean \pm sem, $n=3$ ). (c) Western blot analysis of E-cadherin protein level after transient repression of TP53, TP73 or both concomitantly. Cells were transfected with 60 pmol siRNA and analysis was performed after $48 \mathrm{~h}$. E-cadherin was detected and $\beta$-actin was used as a loading control.

This result was also obtained after expression of $\triangle N p 73$ in HCT116 and in non-invasive SK-Mel-28 cells (Fig. 3.33b). As a marker for high motility and invasion, the protein levels of E-cadherin were determined after transient repression of TP53 and TP73. Western blot analysis revealed a strong decrease in E-cadherin protein level after simultaneous repression of TP53 and TP73 but not after sole loss of TP53 or TP73 (Fig. 3.33c).

\subsubsection{In Vivo Analyses Of Invasiveness Of HCT116 Cells After Loss Of TP53 And TP73}

In order to study the invasive phenotype of the HCT116-TP53-/TP73sh cells in vivo, chorion allantoic membrane (CAM) assays were performed. CAM assays are an established animal model for cancer research and are widely used to study angiogenesis as well as tumor cell migration and invasion (Deryugina \& Quigley 2009). Briefly, fertilized chicken eggs are opened and the CAM becomes accessible. Cells are applied onto the CAM and can be analyzed regarding their growth behavior on and through the membrane.

HCT116 control shRNA, HCT116-TP53/-, HCT116-TP73sh and HCT116-TP53/-/TP73sh cells were applied onto the CAM and the grown tumors including the CAM were isolated after seven days. Tumors were fixed and analyzed regarding their overall growth characteristics. Here, the tumors derived from HCT116-TP53 ${ }^{-/} / T P 73 s h$ macroscopically differed from the tumors derived from the single knock down cells. Whereas the control shRNA transfected 
HCT116 cells and the cells repressed of either TP53 or TP73 exhibited a polypoid cauliflower-like growth, the cells repressed of both TP53 and TP73 exhibited an ulcerative invasive phenotype (Fig. 3.34a), which is often found in invasive carcinoma of the colon.

a

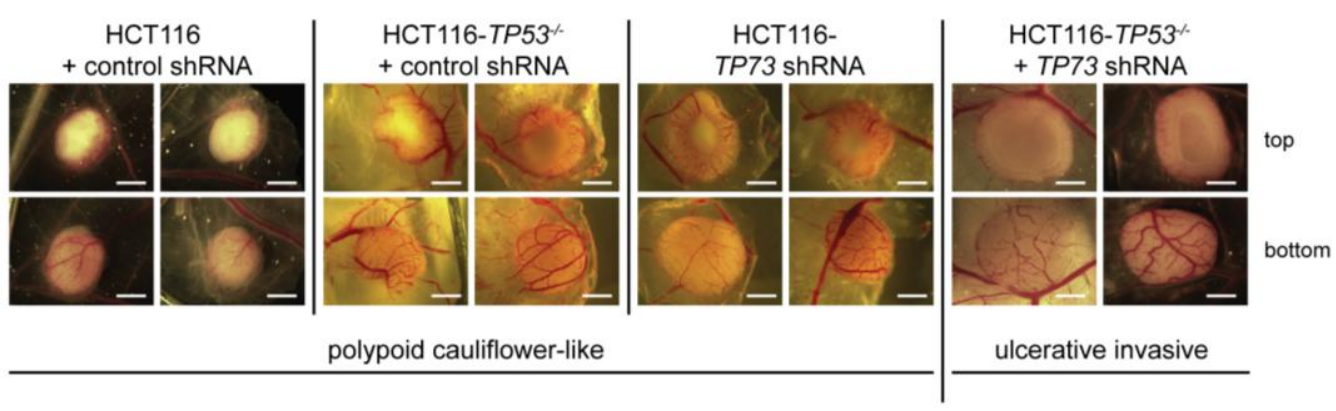

b

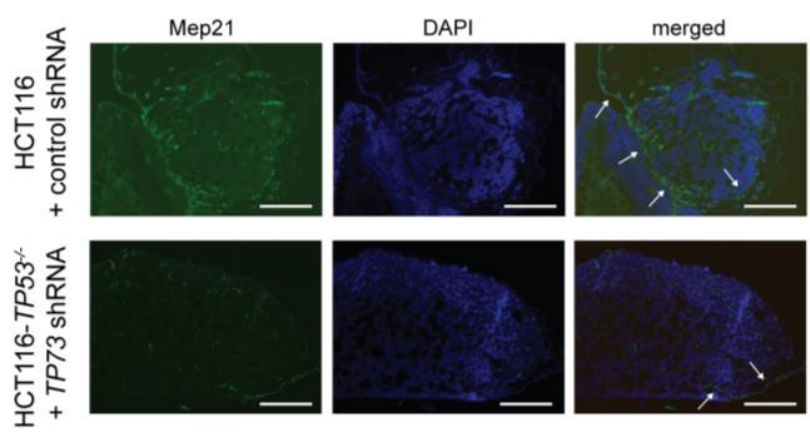

Figure 3.34: Loss of TP53 and TP73 causes ulcerative invasive tumor growth in vivo. (a) CAM assays of HCT116 control shRNA, HCT116-TP53/- control shRNA, HCT116-TP73shRNA and HCT116-TP53 $3^{-/} /$TP73shRNA cells. Two million cells were applied on the CAM on day ten of chicken development. After additional seven days, tumors were isolated and macroscopically analyzed. Two representative tumors derived from each cell line are shown from the top and from the bottom view. Scale bar $200 \mu \mathrm{m}$. (b) Dissected tumors derived from HCT116 control cells and HCT116-TP53/TP73sh cells were sliced into $12 \mu \mathrm{M}$ sections and stained for Mep21 and DAPI. Arrows indicate the CAM. Scale bar $100 \mu \mathrm{m}$

Immunofluorescence microscopy of cryo-sections of HCT116 control shRNA cells and HCT116-TP53 $3^{-/}$TP73sh cells gave more insights into the tumor phenotypes. Dissected tumors were sliced into $12 \mu \mathrm{M}$ sections and stained for Mep21 and DAPI. Staining for Mep21 allowed the discrimination between tumor and blood vessels of the CAM, since Mep21 is a marker for blood endothelial cells of the chicken. Analyses of the tumors derived from HCT116 control cells confirmed the exophytic appearance. The CAM was mainly preserved, but still, the tumor protrudes into the CAM in some sections. Furthermore, the tumor exhibited a very compact and dense structure. In contrast, tumors derived from HCT116-TP53 $\%$ TP73sh cells showed a mostly destroyed CAM and highly invasive tumor growth. Also, the tumor appeared to be very porous.

These in vivo tumor growth analyses confirmed a highly invasive phenotype induced by the loss of both TP53 and TP73. 


\section{Discussion}

\subsection{Increased Activity Of TRIO-Rac1-Arp2/3 Pathway As A Trigger For Migration And CIN}

The development of genomic instability is a hallmark of human cancers, which enables the development of certain cancer hallmarks like insensitivity to anti-growth signals, deregulated apoptotic signaling or tissue invasion and metastasis (Hanahan \& Weinberg 2000; Hanahan \& Weinberg 2011). These newly obtained phenotypes are thought to be acquired by mutations, structural alterations of the genome or chromosomal instability. Thus CIN is associated with tumor progression and an aggressive tumor behavior, which is also characterized by infiltration of nearby tissues and metastases. However, the underlying mechanisms are still largely unknown and a clear link between CIN and cancer cell invasion and metastases is missing so far.

In this thesis, the migration and invasion potential of a panel of MIN/MSI and CIN CRC cell lines and of a panel of melanoma cell lines was examined. Interestingly, not all analyzed CIN cell lines exhibited a migratory and invasive phenotype, but vice versa the migratory and invasive cell lines were CIN (Fig. 3.1, Fig. 3.2, Fig. 3.5). Importantly, the migration and invasion potentials of CRC and melanoma cell lines were strongly associated with increased interphase microtubule plus-end assembly rates (Fig. 3.1, Fig. 3.2). Recently, our group established increased microtubule plus-end assembly rates during mitosis as a common cause for CIN (Ertych et al. 2014), but the role of increased interphase microtubule plus-end assembly rates has not been investigated yet. This apparent new relationship between microtubule plus-end dynamics in interphase and cell migration was surprising. Therefore, it was of great interest to investigate how enhanced microtubule dynamics contribute to the regulation of cell motility.

In this study, a hyperactive TRIO-Rac1-Arp2/3 pathway was identified as a trigger for the development of a highly migratory and a chromosomally instable phenotype. Furthermore, the studies revealed increased microtubule plus-end assembly rates as an activator of this pathway. During interphase, the hyperactivation of TRIO-Rac1-Arp2/3 due to elevated microtubule plus-end assembly rates presumably results in highly branched actin filaments, which are required for lamellipodia and invadopodia formation during cellular migration and tissue invasion. On the other hand, during mitosis, the microtubule-dependent hyperactivation of TRIO-Rac1-Arp2/3 induces spindle misorientation, the generation of lagging chromosomes and CIN. Therefore, increased microtubule plus-end assembly rates promote the development of a highly aggressive and invasive phenotype. 


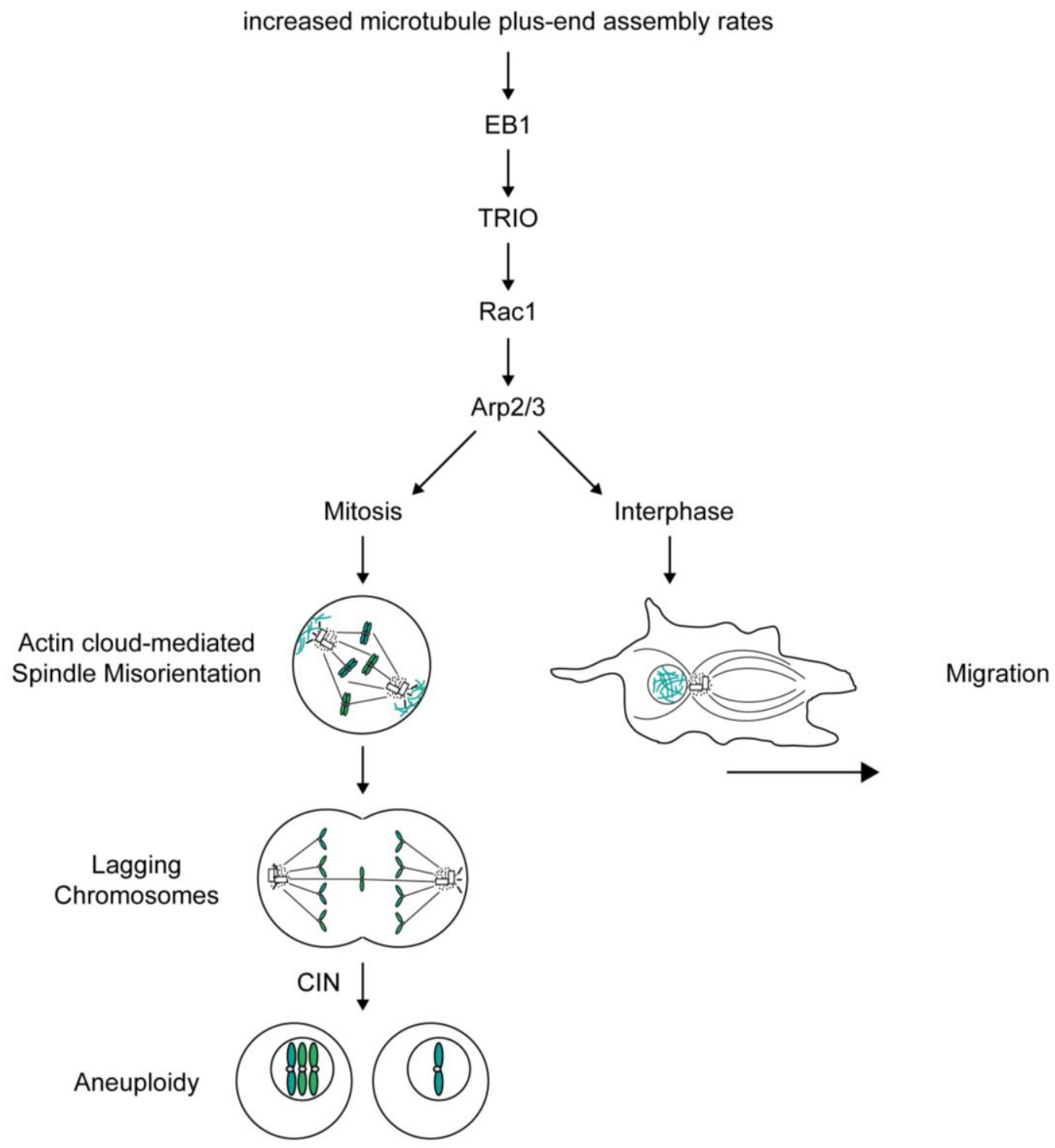

Fig. 4.1: Model showing the microtubule-dependent hyperactivity of the TRIO-Rac1-Arp2/3 pathway that affects both mitosis and interphase. During interphase, in cells exhibiting increased microtubule plus-end assembly rates, TRIO binds to microtubules via EB1 and becomes hyperactivated. TRIO activates Rac1, which subsequently leads to Arp2/3-mediated reorganization of the actin cytoskeleton and the formation of lamellipodia thus facilitating migration and invasion. During mitosis, the microtubule dependent increased activity of the Arp2/3 complex leads to spindle misorientation due to enhanced formation of actin clouds. The spindle misorientation causes lagging chromosomes and CIN.

During cell migration, the activation of Rho family GTPases is mediated by GEFs. At least 20 GEFs are known to regulate the activation of Rac1 by catalyzing the exchange of GDP for GTP (Marei \& Malliri 2016). Upon them TIAM1, TIAM2, GEF-:H1, ASEF and TRIO are well characterized (Kawasaki et al. 2000; Blangy et al. 2000; Krendel et al. 2002; Rooney et al. 2010). TRIO was identified as a microtubule plus-end binding protein in HeLa and neurite cells, whereby binding to microtubules is mediated by the +TIP EB1 (Van Haren et al. 2014). EB1 was described to be an important protein in the development of a signaling platform at the microtubule plus-end (Vaughan 2005; Tamura \& Draviam 2012), which includes binding 
of CLIP-170 (Lantz \& Miller 1998), APC, CLASP1 and CLASP2 (Tsvetkov et al. 2007). Interestingly, $E B 1$ is overexpressed in different tumor types and is associated with increased tumor formation (Wang et al. 2005). Repression of EB1 interfered with formation of lamellipodia protrusions in mouse melanoma cells and deregulated Arp2/3 activity during interphase (Schober et al. 2009). The binding of TRIO to EB1 enhances its activity. Therefore, the question arises, how binding of TRIO and other GEFs to microtubule plusends leads to their activation. In HeLa and neurite cells it was shown, that TRIO binds to microtubules via the navigator complex Nav1 and EB1. Thereby, the GEF activity of TRIO and subsequent neurite outgrowth were enhanced. It is hypothesized, that due to binding of TRIO to Nav1 and EB1, the GEF domain of TRIO becomes exposed and the interaction between TRIO and its target GTPase is stabilized (Van Haren et al. 2014). Further, the binding of TRIO to microtubules via EB1 might lead to a spatial regulated intracellular signaling, which is needed for Rac1-mediated lamellipodia formation at the leading edge of a migrating cell. Microtubule polymerization sequesters and inactivates GEFs whereas microtubule depolymerization releases GEFs (Chang et al. 2007). These processes mainly occur at the microtubule plus-ends. During cell motility, the centrosome and the majority of microtubules are oriented towards the leading edge of the cell (Wittmann \& Waterman-Storer 2001). Furthermore, microtubule plus-ends, which are oriented towards the leading edge, grow more persistently compared to microtubules oriented towards the rear of a cell (Waterman-Storer \& Salmon 1997; Ballestrem et al. 2000). However, microtubules usually do not enter the lamellipodium except some so called 'pioneer microtubules' (Wittmann et al. 2003). It seems possible that TRIO might be bound to microtubule plus-ends via EB1 and is mainly transported towards the leading edge. Here, microtubule depolymerization would lead to a release of TRIO, which would then in turn be able to activate membrane bound Rac1 in the leading edge, resulting in Arp2/3-activation and actin branching within the lamellipodium. It remains unclear, whether TRIO directly activates Rac1 or whether also another Rho-family small GTPase, RhoG, which is also a target of TRIO's GEF activity (Blangy et al. 2000), is involved. RhoG is able to activate both Rac1 and Cdc42 in the lamellipodium in a microtubule dependent manner (Gauthier-Rouviere et al. 1998), resulting in elevated levels of active Rac1 and active Cdc42. Active Rac1 and Cdc42, in turn, lead to WAVEMASPmediated activation of the Arp2/3 complex. The Arp2/3 complex is a well established complex in the formation of branched actin in the lamellipodium, thereby providing a protrusive force (Zheng et al. 2008). The hyperactivity of the Arp2/3 complex contributed to growth and invasiveness of gastric carcinoma (Zheng et al. 2008), whereas silencing interfered with cell migration in pancreatic cancer (Rauhala et al. 2013). Therefore, the hyperactivation of Arp2/3 mediated by the TRIO-Rac1 axis constitutes a greatly important step in tumor cell migration and invasion. 
Because only few microtubules reach into the lamellipodium it is questionable whether they are sufficient to transport enough TRIO molecules for the activation of either Rac1 or RhoG to the side of protrusion. Therefore, an amplification of the signaling cascade might be necessary. Rac1 activity can also be stimulated through the Ras GTPase activating like protein IQGAP1, which stabilizes and increases active Rac1 and Cdc42 levels (Briggs \& Sacks 2003). IQGAP1 binds to the +TIP CLIP-170, which was detected at microtubules in the leading edge (Fukata et al. 2002; Watanabe et al. 2004). Furthermore, IQGAP1 is also localized at the cell cortex. A positive feedback loop between IQGAP1 and Rac1 is described: cortical IQGAP1 stimulates Rac1, which in turn promotes microtubule-dependent stabilization of IQGAP1 (Siegrist \& Doe 2007). Further, not only TRIO but also other GEFs, like GEF-H1 and ASEF might be delivered to the leading edge. Therefore, different GEF activities and IQGAP1 might contribute to the microtubule-dependent activation of Rac1 in the leading edge and an amplified signaling (Fig. 4.2).
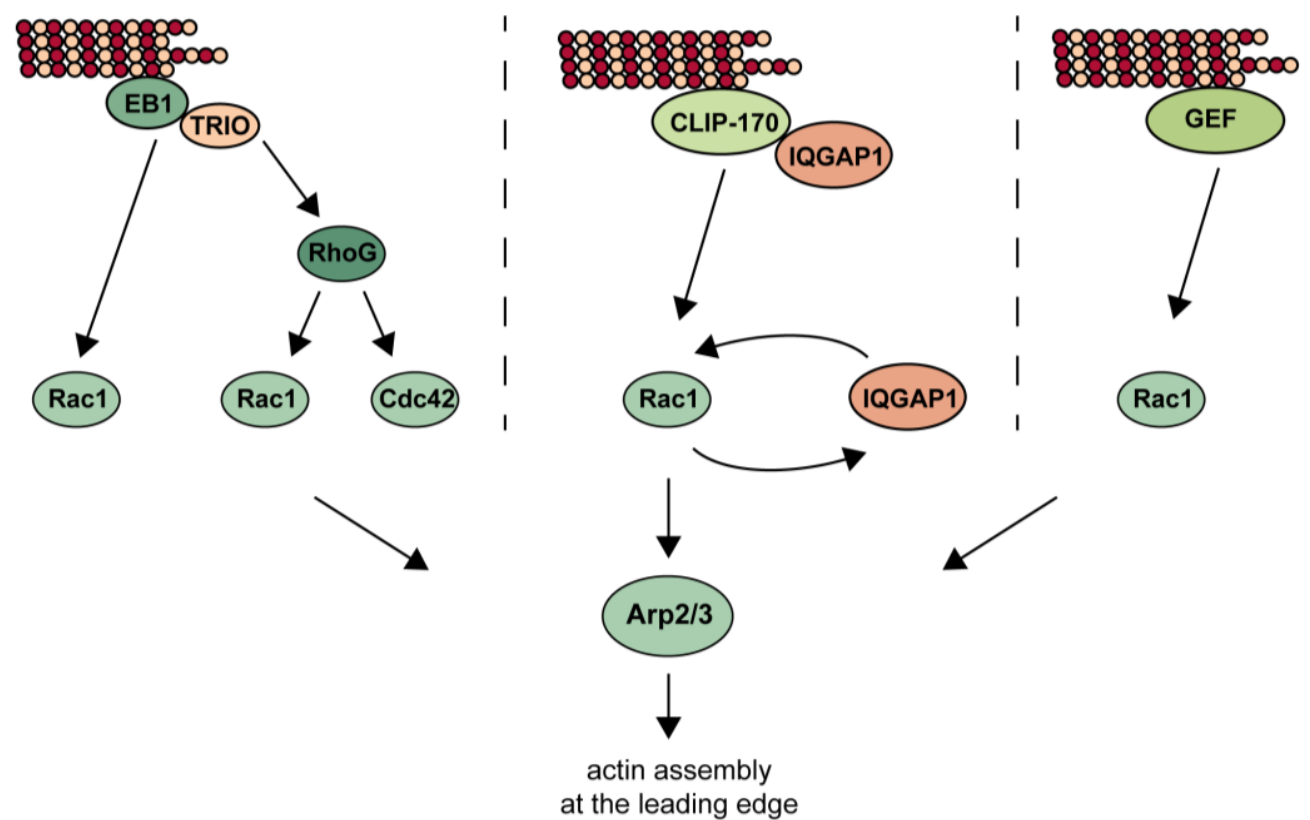

Fig. 4.2: Possible modes of induction of microtubule dependent actin assembly at the leading edge. Microtubule-dependent transport of TRIO to the leading edge might directly activate membrane bound Rac1. But Rac1 activation may also happen due to RhoG activation. Furthermore, IQGAP1 could be transported to Rac1, where a positive feedback loop between Rac1 and IQGAP1 would result in highly active Rac1. Additionally, microtubules could also deliver Rac1-GEFs other than TRIO to the leading edge. These different modes might cooperate to mediate actin assembly through the activation of the Arp2/3 complex.

Recently, also microtubules themselves were described to be capable of actin polymerization (Henty-Ridilla et al. 2016). Also here, the +TIP CLIP-170 is involved. It binds to formins, which accelerate actin polymerization by binding to the growing barbed end (Evangelista et 
al. 2003). Therefore, also TRIO-independent mechanisms could contribute to actin assembly at the leading edge to promote migration.

Interestingly, recent studies linked the amplification of centrosomes with the hyperactivation of Rac1 and an invasive phenotype (Godinho et al. 2014). Thereby, an increased number of centrosomes is thought to nucleate increased amounts of microtubules that activate Rac1 by an unknown mechanism. In conformity with my results, an increased microtubule polymer mass might also expose more microtubule plus-ends with, where binding of TRIO can occur. Therefore, more TRIO could be transported towards Rac1 at the invasive front. Whether increased microtubule plus-end assembly rates, which constitute the trigger of increased Rac1-activity in my studies, also lead to increased microtubule polymer mass is not clear and remains to be studied.

In contrast to interphase, the role of TRIO, Rac1 and the Arp2/3 complex in mitotic chromosome segregation is not established, but some reports hint to mitotic roles of different GEFs. The Rac1-GEF TIAM1 was described to play a role during centrosome separation (Woodcock et al. 2010). Its depletion resulted in increased centrosome separation and chromosome congression failures (Woodcock et al. 2010). Also TRIO was identified as a mitotic Rac1-GEF, acting during cytokinesis at the cleavage furrow (Cannet et al. 2014). Unpublished data from our group showed that TRIO is localized to microtubule plus-ends during interphase and mitosis in an EB1-dependent fashion. At the cell cortex, TRIO might be able to activate Rac1 with subsequent actin polymerization triggered by the Arp2/3 complex. During mitosis, a dynamic, revolving cluster of actin filaments was described to be present from prometaphase to anaphase (Mitsushima et al. 2010). This cluster, referred to as 'actin clouds', is located at the cell cortex, extends into the cytoplasm and depends on Arp2/3 activity (Mitsushima et al. 2010). Actin clouds might exert pulling forces on centrosomes towards retraction fibers, thus polarizing the cell and orientating the mitotic spindle at the longest cell axis (Fink et al. 2011). Unpublished data from our group indeed revealed a decreased formation of actin clouds upon treatment with TRIO-, Rac1- or Arp2/3inhibitors. Thus, the appearance of a hyperactive TRIO-Rac1-Arp2/3 pathway during mitosis might result in an enhanced branched actin polymerization, leading to the accumulation of dense actin clouds. However, it remains unclear, how actin clouds affect spindle positioning. First hints towards this question were provided recently by the Pellman Group (Kwon et al. 2015). Their studies suggest that the actin cloud might exert pulling forces via the microtubule binding myosin Myo10, which was shown to link actin and microtubules and move the spindle poles towards actin clouds and retraction fibers (Weber et al. 2004; Woolner et al. 2008; Kwon et al. 2015). Myo10 was described to exhibit overlapping functions with dynein in mammalian cells. Dynein is mainly involved in anchoring the mitotic spindle at the cell cortex via a complex of $G_{a i}$ LGN-NuMA and a loss of dynein causes 
misorientated mitotic spindles (Kiyomitsu \& Cheeseman 2012). But the cortical distribution of dynein is also affected by external stimuli (Morin \& Bellaïche 2011). Therefore, it is not clear, whether actin clouds influence the localization of dynein or whether dynein affects the orientation of actin clouds.

Our unpublished data showed that the mitotic localization of TRIO is not restricted to astral microtubules, which grow towards the cell cortex and could possibly interact with membrane bound Rac1. Therefore, TRIO might not only function at the cortex but also at centrosomes, since its localization depends on EB1, which was shown to localize also to centrosomes (Morrison et al. 1998; Berrueta et al. 1998; Mimori-Kiyosue et al. 2000). Furthermore, Rac1 was also described to localize to centrosomal regions in early mitosis (Woodcock et al. 2010). Here, it was shown, that the Rac1-GEF TIAM1 facilitates bipolar spindle assembly (Woodcock et al. 2010). Therefore, also TRIO might interact with Rac1 at centrosomes thus triggering actin cloud assembly. Besides microtubules, also actin is important for centrosome separation, since depolymerization of actin inhibits splitting of centrosomes (Uzbekov et al. 2002). Tyrosine-phosphorylated cortactin (p-cortactin) was identified as a trigger for centrosome separation (Wang et al. 2008). Actin filaments can attach to p-cortactin and exert forces to push the centrosomes apart (Wang et al. 2008). During interphase, the phosphorylation of cortactin requires Rac1 activity (Head et al. 2003). Therefore, also during mitosis, active Rac1 might be important in facilitating cortactin phosphorylation. An abnormal regulated cortactin phosphorylation due to Rac1 hyperactivity might further be associated with deregulated spindle formation and chromosome missegregation.

The overexpression and increased activity of TRIO, Rac1 and Arp2/3 is linked to several tumor types. TRIO overexpression, which comes along with hyperactive TRIO, is observed in different cancer types and can be found in the most aggressive forms of malignant glioblastoma, where it is associated with poor patient survival (Schmidt \& Debant 2014). Additionally, our unpublished results revealed a correlation of high TRIO expression in metastases of colon cancers exhibiting CIN. Thus, TRIO is highly associated with both, invasiveness and aneuploidy in late stage colon tumors. Furthermore, deregulated Rac1activity is common in cancer. Rac1 overexpression can be found in breast carcinoma, nonsmall cell lung carcinoma and gastric carcinoma and results in accelerated tumor progression and high mortality rates (Espina et al. 2008). Rac1-signaling modulates cell migration and invasion by mediating lamellipodia formation and MMP expression, thereby leading to tumor metastases (Mack et al. 2011) but a role of hyperactive Rac1 is not directly associated with CIN so far. Downstream of Rac1, also overexpression of the Arp2/3 complex is associated with highly invasive tumors. In gastric carcinoma, increased activity of the Arp2/3 complex is involved in pathogenesis and progression (Zheng et al. 2008). However it remains to be shown whether TRIO, Rac1 or Arp2/3 overexpressing tumors also display 
increased actin cloud formation during mitosis and thus, spindle misorientation and CIN. Still, this shared trigger for the development of CIN and cancer cell migration and invasion may constitute an interesting target in cancer therapies. The Rac1 inhibitor NSC23766 was already used in treatment of different breast cancers, where it induced cell cycle arrest and apoptosis (Yoshida et al. 2010). However, the efficacy of NSC23766 was not sufficient for its substantial clinical use (Bid et al. 2013). But the development of a drug that targets the TRIORac1-Arp2/3 pathway might possibly suppress CIN and cancer cell migration and invasion and thus the two highly relevant phenotypes, which are detected in aggressive cancer.

\section{2 p53 And p73 Act As Regulators Of Chromosomal Stability And Cell Invasion}

In human cancer, the TP53 gene encoding for the tumor suppressor p53 is the most frequently mutated gene. Mutations in TP53 occur late during cancer progression (Baker et al. 1990). p53 has two homologs, p63 and p73. Interestingly, the chromosome region coding for p73 is lost in a wide array of tumors, including neuroblastoma (Kaghad et al. 1997), melanoma (Dracopoli et al. 1989) and hepatocellular carcinoma (Yeh et al. 1994). p73 was shown to inhibit aneuploidy in the absence of p53 in mice (Talos et al. 2007). Furthermore, a dominant negative form of $\mathrm{p} 73, \Delta \mathrm{Np} 73$, which inhibits p53's and p73's function, is often overexpressed in tumors and is associated with a highly metastatic phenotype (Steder et al. 2013). Thus, we wanted to elucidate how p53 and p73 cooperate in maintaining genomic stability and suppressing invasion.

In this study, p53 and p73 were identified as important regulators of chromosomal stability and cellular migration and invasion. Importantly, the concomitant loss of TP53 and TP73 induced aneuploidy (Fig. 3.26d). A complete loss of TP53 is not common in cancer, but several hot spot mutations of p53 are described (Goldstein et al. 2011; Muller \& Vousden 2013). These mutants were shown to interact with $p 73$, thereby inhibiting its transcriptional activity (Gaiddon et al. 2001). But during my studies, an effect of mutant p53 (p53 R175H) onto chromosomal stability was not detected. However, in combination with TP73 repression, the development of CIN was observed (Fig. 3.29). A dominant negative form of $p 73, \Delta N p 73$, was described to negatively regulate both, p53 and p73 function (Grob et al. 2001). In my studies, $\Delta N p 73$ overexpression indeed caused increased amounts of cells displaying lagging chromosomes (Fig. 3.30), which constitute a prerequisite for the development of CIN suggesting that concurrent loss of p53 and p73 function is required to induce CIN.

The loss of function of p53 in human colon carcinoma cells is not sufficient to induce aneuploidy (Bunz et al. 2002). However, in colorectal cancer CIN is often associated with p53 loss. Since p53 and p73 exhibit functional and structural similarities, such as a highly conserved DNA-binding domain (Levrero et al. 2000), they might be able to fulfill redundant 
functions and partially transactivate the same genes. Therefore, upon loss of either p53 or p73, the remaining protein might be able to substitute for the other. This is in agreement with findings from Talos et al., who showed that p73 suppresses polyploidy in the absence of functional p53 in mouse embryonic fibroblasts (MEFs) (Talos et al. 2007). But while these MEFs developed polyploidy, in my studies the used colorectal cancer cells developed aneuploidy. Thus, on the one hand the maintenance of chromosomal stability seems to be regulated by $\mathrm{p} 53$ and $\mathrm{p} 73$ but on the other hand different mechanism might account for that in mice and human.

Further, the absence of p53 was shown to correlate with centrosome amplification in mice (Tarapore \& Fukasawa 2002). Additionally, in mouse models, a relationship between mutant p53 and p73 was described causing overduplication of centrosomes. In these studies, p53 $\mathrm{R} 172 \mathrm{H}$ derived tumors were highly aneuploid due to inhibition of $\mathrm{p} 73$ and subsequent centrosome amplification (Murphy et al. 2000). Supernumerary centrosomes generate lagging chromosomes, due to transient spindle geometry defects occurring during the assembly of multipolar spindles, that are clustered into a spindle pole. Also human tumors show a strong association between mutant p53 and supernumerary centrosomes, including breast tumors (Carroll et al. 1999). However, it was also shown, that loss of p53 alone neither lead to centrosome amplification nor to CIN (Lengauer et al. 1997; Bunz et al. 2002). In addition to deregulated mitotic functions, also erroneous replication might result from simultaneous loss of function of p53 and p73. An aberrant activation of CDK-cyclin complexes during S-phase was indeed detected in mice upon loss of p53 and p73 (Talos et al. 2007). This resulted in aneuploidy due to a delayed S-phase and re-replication. Indicative for erroneous pre-mitotic defects is the occurrence of acentric chromosomes or chromosome bridges (Burrell et al. 2013). Since in my studies, cells concomitantly depleted of TP53 and TP73 exhibited high amounts of cells displaying anaphase bridges (Fig 3.25d), it might be possible that replication stress leads also to structural chromosome aberrations (S-CIN) in these cells. Thus, depletion of TP53 and TP73 might cause replication stress, which affects the following mitosis (Wilhelm et al. 2013; Gelot et al. 2015). Recently, an impaired replication fork progression was detected in the absence of p53 (Yeo et al. 2016). Thereby, p53 was discovered to prevent conflicts between transcription and replication in S-phase. Upon loss of p53, the timely progression of replication was disturbed by secondary DNA structures, leading to replication fork stalling and collapse (Yeo et al. 2016). This in turn results in DNA double strand breaks, which may underlie deletion in mitosis.

My studies showed that the repression of both, TP53 and TP73 or the overexpression of $\Delta N p 73$ resulted in an invasive phenotype. Furthermore, the loss of both transcription factors led to a strong reduction in the E-cadherin protein level (Fig. 3.32b), which might be a marker for EMT. Interestingly, in vivo studies of HCT116 cells repressed of both TP53 and TP73 by 
using CAM assays revealed a clinical relevant phenotype: while tumors derived from control shRNA transfected HCT116 cells exhibited a polypoid cauliflower-like phenotype, tumors derived from TP73 shRNA transfected HCT116-TP53-- cells exhibited an ulcerative invasive tumor growth (Fig. 3.34).

Wild-type p53 was found to inhibit EMT, cancer cell invasion and metastases by Mdm2mediated degradation of the transcriptional repressor Slug, which leads to enhanced $C D H 1$ (encoding for E-cadherin) expression (Wang et al. 2009). But p53's function is opposed by another transcription factor, Twist, which is able to hinder DNA binding of p53 (Shiota et al. 2008; Smit \& Peeper 2008). Therefore, upon loss of TP53, both Slug and Twist can drive the repression of $C D H 1$ and promote EMT. Furthermore, Slug and Twist were described to be increased upon TP73 repression in MCF-10A cells leading to a highly migratory phenotype of this cell line (Zhang et al. 2012). This indicates a regulation of Slug and Twist by both p53 and p73. Thus, the loss of function of p53 and p73 downregulates $C D H 1$ expression, which is frequently observed in cancer and strongly associated with metastases in mouse models (Derksen et al. 2006).

During invasion, the formation of podosomes and invadopodia is an important step. These membrane protrusions are involved in adhesion and invasion and are capable of secretion of matrix-metalloproteinases (MMPs). The formation of podosomes is mediated by Src kinase activity, which is counteracted by p53. Hence, wild-type p53 is involved in suppressing the formation of invadopodia and podosomes. This is mediated by up-regulating PTEN and caldesmon. PTEN antagonizes phosphatidylinositol-3,4,5-trisphosphate $\left(\mathrm{PIP}_{3}\right)$ and phosphatidylinositol-4,5-bisphosphate $\left(\mathrm{PIP}_{2}\right)$ formation during cell polarization, whereas caldesmon inhibits Arp2/3 induced actin polymerization and also stabilizes actin stress fibers (Yamakita et al. 2003; Yoshio et al. 2007).

The loss of function of $p 53$ and p73 can also occur due to overexpression of $\Delta N p 73$. This overexpression results in the gain of highly aggressive traits in cancer (Engelmann et al. 2014). $\triangle$ Np73 inhibits p53 and p73 and therefore might work antagonistically to both (Pützer 2013). $\Delta N p 73$ overexpression is found in several cancers including lung cancer (Uramoto et al. 2004), neuroblastoma (Douc-Rasy et al. 2002) and prostate carcinoma (Guan \& Chen 2005). The $\triangle \mathrm{Np} 73$ isoform is a regulator of EMT by up-regulating $\mathrm{N}$-cadherin and vimentin as well as Slug, which represses $C H D 1$, and was found highly expressed in metastases of skin cancer patients (Engelmann et al. 2014). Vice versa, the knock down of $\triangle N p 73$ in metastatic cell lines suppressed their invasion potential (Steder et al. 2013). Thus $\Delta N p 73$ plays an important role in metastasis induction potentially by inhibiting the repressive functions of p53 and p73. The loss of function of p53 and p73 were accompanied by increased mitotic and interphase microtubule plus-end assembly rates. Based on the findings represented in this thesis, repression of TP53 and TP73 might hyperactivate the TRIO-Rac1- 
Arp2/3 pathway, which in turn leads to a highly invasive phenotype. Thus, the increased microtubule plus-end assembly rates in mitosis and interphase detected after depletion of TP53 and TP73 or overexpression of $\triangle N p 73$ might support the hyperactivation of TRIO and the respective downstream signaling. This might be in line with the observation that the loss of TP53 alone is not sufficient to induce migration and invasion (Sablina et al. 2003; Guo \& Zheng 2004) (Fig. 3.32a), since depletion of TP53 alone did not induce increased microtubule plus-end assembly rates (Fig. 3.25b). In mice, TP53 deletion was described to result in elevated activities of the PI3K-Rac1-Akt signaling pathway without altering their protein expression (Guo et al. 2003). This suggests that cells exhibit enhanced capability of cell migration upon loss of function of p53 but nevertheless also other stimuli are necessary for the development of a migratory and invasive phenotype. These additionally required stimuli could involve various alterations, which affect and enhance microtubule plus-end assembly rates. This may include mutations of TP53. Mutations of TP53 can result in gain of functions, which predispose cells to cancer invasion and metastasis (Oren \& Rotter 2010). In mouse models, mutant p53 increases the capability of metastatic carcinomas (Lang et al. 2004; Heinlein et al. 2008).

RNA-sequencing revealed a pronounced repressed expression of CDKN1A upon depletion of TP53 and TP73, which was confirmed by qRT-PCR analyses (Table 3.1, Fig. 3.31a). Furthermore, the re-expression of CDKN1A suppressed microtubule plus-end assembly rates in interphase detected upon loss of p53 and p73 (Fig. 3.32b, d). Thus it might be possible, that the observed phenotypes are mediated by p21. p21 acts as a CDK inhibitor, and therefore the loss of function of p21 would lead to an increased CDK activity. Known p21 targets are CDK2 in interphase and CDK1 in mitosis. Whereas CDK2 functions mainly during $\mathrm{G}_{1}$-S-phase, CDK1 is active during mitosis. A function of CDKs in the regulation of microtubule dynamics is not known, but a few reports have shown a localization of CDKs at centrosomes. For instance, the mitotic CDK1 is transported to the centrosome by the centrosomal protein Cep63 (Löffler et al. 2011) and becomes activated there in early mitosis (Jackman et al. 2003). Further, CP110 is a target of CDK2, which is involved in centrosome duplication. Loss of CDK-phosphorylation on CP110 led to polyploidy (Chen et al. 2002). Also the spindle checkpoint kinase Mps1 is phosphorylated by CDK2 (Fisk \& Winey 2001). Moreover, hyperactivation of CDK2 correlates with centrosome amplification (Adon et al. 2010). Thus, a relationship between deregulated CDK activity and dysfunction might exist. Therefore, loss of p21 might affect centrosome-mediated microtubule dynamics through increased CDK activity. Whether p21 indeed exerts its microtubule dynamic regulating function via its CDK-inhibiting activity needs to be further analyzed in future studies. 


\section{References}

Abbas, T. \& Dutta, A., 2009. P21 in Cancer: Intricate Networks and Multiple Activities. Nature Reviews Cancer, 9(6), pp.400-414.

Achard, V. et al., 2010. A "Primer"-Based Mechanism Underlies Branched Actin Filament Network Formation and Motility. Current Biology, 20, pp.423-428.

Adachi, A. et al., 1987. Productive, persistent infection of human colorectal cell lines with human immunodeficiency virus. Journal of Virology, 61(1), pp.209-213.

Adon, A.M. et al., 2010. Cdk2 and Cdk4 regulate the centrosome cycle and are critical mediators of centrosome amplification in p53-null cells. Molecular and cellular biology, 30(3), pp.694-710.

Akhmanova, A. \& Steinmetz, M.O., 2015. Control of microtubule organization and dynamics: two ends in the limelight. Nature Reviews Molecular Cell Biology, 16(12), pp.711-26.

Akin, O. \& Mullins, R.D., 2008. Capping Protein Increases the Rate of Actin-Based Motility by Promoting Filament Nucleation by the Arp2/3 Complex. Cell, 133(5), pp.841-851.

Alla, V. et al., 2010. E2F1 in melanoma progression and metastasis. Journal of the National Cancer Institute, 102(2), pp.127-133.

Amundson, S.A., Myers, T.G. \& Fornace, A.J.J., 1998. Roles for p53 in growth arrest and apoptosis: putting on the brakes after genotoxic stress. Oncogene, 17(25), pp.3287-99.

Arellano, M. \& Moreno, S., 1997. Regulation of CDK/cyclin Complexes During the Cell. International Journal of Biochemistry and Cell Biology, 29(559-573).

Baker, S.J. et al., 1990. P53 Gene Mutations Occur in Combination With 17P Allelic Deletions As Late Events in Colorectal Tumorigenesis. Cancer Research, 50(23), pp.77177722.

Bakhoum, S.F. et al., 2009. Genome stability is ensured by temporal control of kinetochoremicrotubule dynamics. Nature cell biology, 11(1), pp.27-35.

Bakhoum, S.F. et al., 2014. The mitotic origin of chromosomal instability. Current Biology, 24(4), pp.R148-R149.

Bakhoum, S.F. \& Compton, D.A., 2012. Chromosomal instability and cancer: a complex relationship with therapeutic potential. The Journal of Clinical Investigation, 122(4), pp.11381143. 
Ballestrem, C. et al., 2000. Actin-dependent lamellipodia formation and microtubuledependent tail retraction control-directed cell migration. Molecular biology of the cell, 11(9), pp.2999-3012.

Banin, S. et al., 1998. Enhanced Phosphorytation of p53 by ATM in Response to DNA Damage. Science, 281(5383), pp.1674-1677.

Barber, T.D. et al., 2008. Chromatid cohesion defects may underlie chromosome instability in human colorectal cancers. Proceedings of the National Academy of Sciences of the United States of America, 105(9), pp.3443-3448.

Bartek, J., Lukas, C. \& Lukas, J., 2004. Checking on DNA damage in S phase. Nature reviews. Molecular cell biology, 5(10), pp.792-804.

Bergstralh, D.T. \& St Johnston, D., 2014. Spindle orientation: What if it goes wrong? Seminars in Cell and Developmental Biology, 34, pp.140-145.

Berrueta, L. et al., 1998. The adenomatous polyposis coli-binding protein EB1 is associated with cytoplasmic and spindle microtubules. Proceedings of the National Academy of Sciences of the United States of America, 95(18), pp.10596-10601.

Bershadsky, A. et al., 1996. Involvement of microtubules in the control of adhesiondependent signal transduction. Current biology, 6(10), pp.1279-1289.

Bershadsky, A.D., Vaisberg, E.A. \& Vasiliev, J.M., 1991. Pseudopodial activity at the active edge of migrating fibroblast is decreased after drug-induced microtubule depolymerization. Cell motility and the cytoskeleton, 19(3), pp.152-158.

Bertoli, C., Skotheim, J.M. \& de Bruin, R.A.M., 2013. Control of cell cycle transcription during G1 and S phases. Nature reviews. Molecular cell biology, 14(8), pp.518-28.

Bid, H.K. et al., 2013. RAC1: an emerging therapeutic option for targeting cancer angiogenesis and metastasis. Molecular cancer therapeutics, 12(10), pp.1925-1934.

Blanchoin, L. et al., 2014. Actin dynamics, architecture, and mechanics in cell motility. Physiological reviews, 94(1), pp.235-63.

Blangy, A. et al., 2000. TrioGEF1 controls Rac- and Cdc42-dependent cell structures through the direct activation of RhoG. Journal of cell science, 113, pp.729-39.

Boettner, B. \& Van Aelst, L., 2002. The role of Rho GTPases in disease development. Gene, 286(2), pp.155-174. 
Bos, J.L., Rehmann, H. \& Wittinghofer, A., 2007. GEFs and GAPs: Critical Elements in the Control of Small G Proteins. Cell, 129, pp.865-877.

Bouquier, N. et al., 2009. A Cell Active Chemical GEF Inhibitor Selectively Targets the Trio/RhoG/Rac1 Signaling Pathway. Chemistry and Biology, 16(6), pp.657-666.

Brattain, M.G. et al., 1984. Heterogeneity of human colon carcinoma. Cancer metastasis reviews, 3, pp.177-191.

Brattain, M.G. et al., 1981. Heterogeneity of Malignant Cells from a Human Colonic Carcinoma. Cancer Research, 41(May), pp.1751-1756.

Bretscher, M.S., 1996. Getting membrane flow and the cytoskeleton to cooperate in moving cells. Cell, 87(4), pp.601-606.

Briggs, M.W. \& Sacks, D.B., 2003. IQGAP proteins are integral components of cytoskeletal regulation. EMBO reports, 4(6), pp.571-4.

Brinkley, B.R., 2001. Managing the centrosome numbers game: From chaos to stability in cancer cell division. Trends in Cell Biology, 11(1), pp.18-21.

Brouhard, G.J. et al., 2008. XMAP215 Is a Processive Microtubule Polymerase. Cell, 132(1), pp.79-88.

Buccione, R., Orth, J.D. \& McNiven, M.A., 2004. Foot and Mouth: Podosomes, Invadopodia and Circular Dorsal Ruffles. Nature Reviews Molecular Cell Biology 5(8), pp.647-657.

Bunz, F. et al., 2002. Targeted Inactivation of p53 in Human Cells Does Not Result in Aneuploidy. Cancer Research, 62, pp.1129-1133.

Burns, K.M. et al., 2014. Nucleotide exchange in dimeric MCAK induces longitudinal and lateral stress at microtubule ends to support depolymerization. Structure, 22(8), pp.11731183.

Burrell, R.A. et al., 2013. Replication stress links structural and numerical cancer chromosomal instability. Nature, 494(7438), pp.492-496.

Burridge, K. \& Wennerberg, K., 2004. Rho and Rac Take Center Stage. Cell, 116(2), pp.167-179.

Busson, S. et al., 1998. Dynein and dynactin are localized to astral microtubules and at cortical sites in mitotic epithelial cells. Current Biology, 8(9), pp.541-544. 
Cain, R.J. \& Ridley, A.J., 2009. Phosphoinositide 3-kinases in cell migration. Biology of the cell, 101(1), pp.13-29.

Calderwood, D.A., Shattil, S.J. \& Ginsberg, M.H., 2000. Integrins and actin filaments: reciprocal regulation of cell adhesion and signaling. The Journal of biological chemistry, 275(30), pp.22607-22610.

Cannet, A. et al., 2014. Identification of a mitotic Rac-GEF, Trio, that counteracts MgcRacGAP function during cytokinesis. Molecular Biology of the Cell, 25(25), pp.40634071.

Carey, T.E. et al., 1976. Cell surface antigens of human malignant melanoma: Mixed hemadsorption assays for humoral immunity to cultured autologous melanoma cells. Immunology, 73(9), pp.3278-3282.

Carroll, P.E. et al., 1999. Centrosome hyperamplification in human cancer: chromosome instability induced by p53 mutation and/or Mdm2 overexpression. Oncogene, 18(11), pp.1935-1944.

Chambers, A.F. \& Matrisian, L.M., 1997. Changing views of the role of matrix metalloproteinases in metastasis. JNCI Journal of the National Cancer Institute, 89(17), pp.1260-1270.

Chan, Y.W. et al., 2012. Aurora B controls kinetochore-microtubule attachments by inhibiting Ska complex-KMN network interaction. Journal of Cell Biology, 196(5), pp.563-571.

Chang, Y.-C. et al., 2007. GEF-H1 Couples Nocodazole-induced Microtubule Disassembly to Cell Contractility via RhoA. Molecular biology of the cell, 19(1), pp.308-317.

Cheeseman, I.M. et al., 2002. Phospho-regulation of kinetochore-microtubule attachments by the Aurora kinase Ipl1p. Cell, 111(2), pp.163-172.

Cheeseman, I.M. et al., 2006. The Conserved KMN Network Constitutes the Core Microtubule-Binding Site of the Kinetochore. Cell, 127(5), pp.983-997.

Cheeseman, I.M. \& Desai, A., 2008. Molecular architecture of the kinetochore-microtubule interface. Nature reviews. Molecular cell biology, 9(1), pp.33-46.

Chen, Z. et al., 2002. CP110, a cell cycle-dependent CDK substrate, regulates centrosome duplication in human cells. Developmental Cell, 3(3), pp.339-350. 
Chew, T.L. et al., 2002. A fluorescent resonant energy transfer-based biosensor reveals transient and regional myosin light chain kinase activation in lamella and cleavage furrows. Journal of Cell Biology, 156(3), pp.543-553.

Cimini, D. et al., 2001. Merotelic kinetochore orientation is a major mechanism of aneuploidy in mitotic mammalian tissue cells. Journal of Cell Biology, 152(3), pp.517-527.

Cimini, D. et al., 2003. Merotelic kinetochore orientation occurs frequently during early mitosis in mammalian tissue cells and error correction is achieved by two different mechanisms. Journal of cell science, 116(Pt 20), pp.4213-4225.

Cimini, D. et al., 2002. Merotelic kinetochore orientation versus chromosome monoorientation in the origin of lagging chromosomes in human primary cells. Journal of cell science, 115(Pt 3), pp.507-515.

Coleman, T.R. \& Dunphy, W.G., 1994. Cdc2 regulatory factors. Current Opinion in Cell Biology, 6, pp.877-882.

Concin, N. et al., 2005. Clinical relevance of dominant-negative p73 isoforms for responsiveness to chemotherapy and survival in ovarian cancer: Evidence for a crucial p53p73 cross-talk in vivo. Clinical Cancer Research, 11(23), pp.8372-8383.

Concin, N. et al., 2004. Transdominant $\triangle T A p 73$ Isoforms Are Frequently Up-regulated in Ovarian Cancer. Evidence for Their Role as Epigenetic p53 Inhibitors in Vivo. Cancer Research, 64(7), pp.2449-2460.

Cook, D.R., Rossman, K.L. \& Der, C.J., 2013. Rho guanine nucleotide exchange factors: regulators of Rho GTPase activity in development and disease. Oncogene, 33(31), pp.40214035.

Cortez, D., 1999. Requirement of ATM-Dependent Phosphorylation of Brca1 in the DNA Damage Response to Double-Strand Breaks. Science, 286(5442), pp.1162-1166.

Coussens, L.M. \& Werb, Z., 1996. Matrix metalloproteinases and the development of cancer. Chemistry \& Biology, 3(11), pp.895-904.

Crainie, M. et al., 1999. Overexpression of the receptor for hyaluronan-mediated motility (RHAMM) characterizes the malignant clone in multiple myeloma: identification of three distinct RHAMM variants. Blood, 93(5), pp.1684-96. 
Dalal, B.I., Keown, P. a \& Greenberg, a H., 1993. Immunocytochemical localization of secreted transforming growth factor-beta 1 to the advancing edges of primary tumors and to lymph node metastases of human mammary carcinoma. The American journal of pathology, 143(2), pp.381-9.

Daub, H. et al., 2001. Rac/Cdc42 and p65PAK regulate the microtubule-destabilizing protein stathmin through phosphorylation at serine 16. Journal of Biological Chemistry, 276(3), pp.1677-1680.

DeLuca, J.G. et al., 2006. Kinetochore Microtubule Dynamics and Attachment Stability Are Regulated by Hec1. Cell, 127(5), pp.969-982.

Deng, C. et al., 1995. Mice lacking p21CIP1/WAF1 undergo normal development, but are defective in G1 checkpoint control. Cell, 82(4), pp.675-684.

Derksen, P.W.B. et al., 2006. Somatic inactivation of E-cadherin and p53 in mice leads to metastatic lobular mammary carcinoma through induction of anoikis resistance and angiogenesis. Cancer Cell, 10(5), pp.437-449.

Deryugina, E.I. \& Quigley, J.P., 2009. Chick embryo chorioallantoic membrane model systems to study and visualize human tumor cell metastasis. Cell, 130(6), pp.1119-1130.

Desai, A. \& Mitchison, T.J., 1997. Microtubule Polymerization Dynamics. Annual review of cell and developmental biology, 13, pp.83-117.

Douc-Rasy, S. et al., 2002. $\Delta \mathrm{N}-\mathrm{p} 73 \alpha$ Accumulates in Human Neuroblastic Tumors. The American Journal of Pathology, 160(2), pp.631-639.

Dracopoli, N.C. et al., 1989. Loss of alleles from the distal short arm of chromosome 1 occurs late in melanoma tumor progression. Proc.Natl.Acad.Sci.USA, 86(12), pp.4614-4618.

Drewinko, B. et al., 1976. Establishment of a human carcinoembryonic antigen-producing colon adenocarcinoma cell line. Cancer research, 36(2 Pt 1), pp.467-75.

Dujardin, D.L. et al., 2003. A role for cytoplasmic dynein and LIS1 in directed cell movement. Journal of Cell Biology, 163(6), pp.1205-1211.

Efimov, A. et al., 2008. Paxillin-dependent stimulation of microtubule catastrophes at focal adhesion sites. Journal of cell science, 121, pp.196-204.

Elbashir, S.M. et al., 2001. Duplexes of 21-nucleotide RNAs meditae RNA interference in cultured mammalian cells. Nature, 411(May), pp.1-5. 
El-Deiry, W.S. et al., 1993. WAF1, a potential mediator of p53 tumor suppression. Cell, 75(4), pp.817-825.

El-sibai, M. et al., 2009. RhoA/ROCK-mediated switching between Cdc42- and Rac1dependent protrusion in MTLn3 carcinoma cells. Experimental Cell Research, 314(7), pp.1540-1552.

Engelmann, D. et al., 2014. A balancing act: orchestrating amino-truncated and full-length p73 variants as decisive factors in cancer progression. Oncogene, (August), pp.1-13.

Ertych, N. et al., 2014. Increased microtubule assembly rates influence chromosomal instability in colorectal cancer cells. Nature cell biology, 16(8), pp.779-91.

Espina, C. et al., 2008. A critical role for Rac1 in tumor progression of human colorectal adenocarcinoma cells. The American journal of pathology, 172(1), pp.156-66.

Etienne-Manneville, S., 2013. Microtubules in cell migration. Annual review of cell and developmental biology, 29, pp.471-99.

Etienne-Manneville, S. \& Hall, A., 2003. Cdc42 regulates GSK-3 b and adenomatous polyposis coli to control cell polarity. Nature, 421(February), pp.753-756.

Evangelista, M., Zigmond, S. \& Boone, C., 2003. Formins: signaling effectors for assembly and polarization of actin filaments. Journal of cell science, 116(Pt 13), pp.2603-2611.

Fife, C.M., Mccarroll, J.A.. \& Kavallaris, M., 2014. Movers and shakers: Cell cytoskeleton in cancer metastasis. British Journal of Pharmacology, pp.5507-5523.

Fink, J. et al., 2011. External forces control mitotic spindle positioning. Nature cell biology, 13(7), pp.771-8.

Fisk, H.A. \& Winey, M., 2001. The mouse Mps1p-like kinase regulates centrosome duplication. Cell, 106(1), pp.95-104.

Friedl, P. \& Wolf, K., 2003. Tumour-cell invasion and migration: diversity and escape mechanisms. Nature reviews. Cancer, 3(5), pp.362-374.

Fukata, M. et al., 2002. Rac1 and Cdc42 capture microtubules through IQGAP1 and CLIP170. Cell, 109(7), pp.873-885.

Gadde, S. \& Heald, R., 2004. Mechanisms and molecules of the mitotic spindle. Current Biology, 14(18), pp.797-805. 
Gaiddon, C. et al., 2001. A subset of tumor-derived mutant forms of p53 down-regulate p63 and p73 through a direct interaction with the p53 core domain. Mol Cell Biol, 21(5), pp.18741887.

Ganem, N.J., Godinho, S.A. \& Pellman, D., 2009. A mechanism linking extra centrosomes to chromosomal instability. Nature, 460(7252), pp.278-82.

Gard, D.L. \& Kirschner, M.W., 1987. A microtubule-associated protein from Xenopus eggs that specifically promotes assembly at the plus-end. Journal of Cell Biology, 105(5), pp.2203-2215.

Gauthier-Rouviere, C. et al., 1998. RhoG GTPase controls a pathway that independently activates Rac1 and Cdc42Hs. Mol.Biol.Cell, 9(6), pp.1379-1394.

Gelot, C., Magdalou, I. \& Lopez, B.S., 2015. Replication stress in mammalian cells and its consequences for mitosis. Genes, 6(2), pp.267-298.

Godinho, S.A. et al., 2014. Oncogene-like induction of cellular invasion from centrosome amplification. Nature, 510(7503), pp.167-171.

Goldstein, I. et al., 2011. Understanding wild-type and mutant p53 activities in human cancer: new landmarks on the way to targeted therapies. Cancer Gene Ther, 18(1), pp.2-11.

Goode, B.L., Drubin, D.G. \& Barnes, G., 2000. Functional cooperation between the microtubule and actin cytoskeletons. Current Opinion in Cell Biology, 12(1), pp.63-71.

Gorbsky, G.J., 2004. Mitosis: MCAK under the Aura of Aurora B. Current Biology, 14(9), pp.346-348.

Grego, S., Cantillana, V. \& Salmon, E.D., 2001. Microtubule treadmilling in vitro investigated by fluorescence speckle and confocal microscopy. Biophysical journal, 81(1), pp.66-78.

Grill, S.W. et al., 2003. The distribution of active force generators controls mitotic spindle position. Science, 301(5632), pp.518-521.

Grob, T.J. et al., 2001. Human delta Np73 regulates a dominant negative feedback loop for TAp73 and p53. Cell death and differentiation, 8(12), pp.1213-1223.

Gruis, N.A. et al., 1995. Genetic evidence in melanoma and bladder cancers that p16 and p53 function in separate pathways of tumor suppression. American Journal of Pathology, 146(5), pp.1199-1206. 
Guan, M. \& Chen, Y., 2005. Aberrant expression of DeltaNp73 in benign and malignant tumours of the prostate: correlation with Gleason score. Journal of clinical pathology, 58(11), pp.1175-1179.

Gundersen, G.G. \& Bulinski, J.C., 1988. Selective stabilization of microtubules oriented toward the direction of cell migration. Proceedings of the National Academy of Sciences of the United States of America, 85(16), pp.5946-50.

Guo, C.P. et al., 2011. Potent anti-tumor effect generated by a novel human papillomavirus (HPV) antagonist peptide reactivating the pRb/E2F pathway. PLoS ONE, 6(3).

Guo, F. et al., 2003. P19Arf-p53 tumor suppressor pathway regulates cell motility by suppression of phosphoinositide 3-kinase and Rac1 GTPase activities. Journal of Biological Chemistry, 278(16), pp.14414-14419.

Guo, F. \& Zheng, Y., 2004. Rho family GTPases cooperate with p53 deletion to promote primary mouse embryonic fibroblast cell invasion. Oncogene, 23(33), pp.5577-85.

Hall, A., 2012. Rho family GTPases. Biochemical Society transactions, 40(6), pp.1378-82.

Hanahan, D. \& Weinberg, R. a., 2011. Hallmarks of cancer: The next generation. Cell, 144(5), pp.646-674.

Hanahan, D. \& Weinberg, R.A., 2000. The hallmarks of cancer. Cell, 100, pp.57-70.

Van Haren, J. et al., 2014. Dynamic microtubules catalyze formation of navigator-TRIO complexes to regulate neurite extension. Current Biology, 24(15), pp.1778-1785.

Harper, J.W. et al., 1995. Inhibition of cyclin-dependent kinases by p21. Molecular biology of the cell, 6(4), pp.387-400.

Harper, J.W. et al., 1993. The p21 Cdk-Interacting Protein Cipl Is a Potent Inhibitor of G I Cyclin-Dependent Kinases. Cell, 75, pp.805-816.

Head, J.A. et al., 2003. Cortactin tyrosine phosphorylation requires Rac1 activity and association with the cortical actin cytoskeleton. Molecular biology of the cell, 14(May), pp.3216-3229.

Heinen, D. et al., 1995. Microsatellite Instability in Colorectal Adenocarcinoma Cell Lines That Have Full-Length Adenomatous Polyposis Coli Protein. Cancer, pp.4797-4799. 
Heinlein, C. et al., 2008. Mutant p53R270H gain of function phenotype in a mouse model for oncogene-induced mammary carcinogenesis. International Journal of Cancer, 122(8), pp.1701-1709.

Henty-Ridilla, J.L. et al., 2016. Accelerated actin filament polymerization from microtubule plus ends. Science, 352(6288).

Hofmann, U.B. et al., 2000. Matrix metalloproteinases in human melanoma. The Journal of investigative dermatology, 115(3), pp.337-344.

Holland, A.J. \& Cleveland, D.W., 2012. Losing balance: the origin and impact of aneuploidy in cancer. EMBO reports, 13(6), pp.501-14.

Holland, G.J. et al., 2009. Sensing Chromosome Bi-Orientation Kinase from Kinetochore Substrates. Science, 323(March), pp.1350-1353.

Hunter, A.W. et al., 2003. The kinesin-related protein MCAK is a microtubule depolymerase that forms an ATP-hydrolyzing complex at microtubule ends. Molecular Cell, 11(2), pp.445457.

Jackman, M. et al., 2003. Active cyclin B1-Cdk1 first appears on centrosomes in prophase. Nature cell biology, 5(2), pp.143-148.

Jordan, M.A. \& Wilson, L., 2004. Microtubules as a target for anticancer drugs. Nature Reviews. Cancer, 4, pp. 253-265

Kaghad, M. et al., 1997. Monoallelically expressed gene related to p53 at 1p36, a region frequently deleted in neuroblastoma and other human cancers. Cell, 90(4), pp.809-819.

Kardon, J.R. \& Vale, R.D., 2009. Regulators of the cytoplasmic dynein motor. Nature reviews Molecular cell biology, 10(12), pp.854-865.

Kaverina, I., Rottner, K. \& Small, J.V., 1998. Targeting, capture, and stabilization of microtubules at early focal adhesions. Journal of Cell Biology, 142(1), pp.181-190.

Kaverina, I. \& Straube, A., 2011. Regulation of cell migration by dynamic microtubules. Seminars in Cell and Developmental Biology, 22(9), pp.968-974.

Kawasaki, Y. et al., 2000. Asef, a link between the tumor suppressor APC and G-protein signaling. Science, 289(5482), pp.1194-1197.

Kessenbrock, K., Plaks, V. \& Werb, Z., 2010. Matrix Metalloproteinases: Regulators of the Tumor Microenvironment. Cell, 141(1), pp.52-67. 
Kim, E. \& Deppert, W., 2004. Transcriptional activities of mutant p53: When mutations are more than a loss. Journal of Cellular Biochemistry, 93(5), pp.878-886.

Kimura, K. et al., 1996. Regulation of myosin phosphatase by Rho and Rho-associated kinase (Rho-kinase). Science, 273(5272), pp.245-248.

Kirschner, M.W. \& Mitchison, T.J., 1986. Beyond self assembly: from microtubules to morphogenesis. Cell, 45, pp.329-342.

Kita, K. et al., 2006. Adenomatous Polyposis Coli on Microtubule Plus Ends in Cell Extensions Can Promote Microtubule Net Growth with or without EB1. Molecular biology of the cell, 17, pp.2331-2345.

Kiyomitsu, T. \& Cheeseman, I.M., 2012. Chromosome- and spindle-pole-derived signals generate an intrinsic code for spindle position and orientation. Nature cell biology, 14(3), pp.311-7.

Knowlton, A.L., Lan, W. \& Stukenberg, P.T., 2006. Aurora B Is Enriched at Merotelic Attachment Sites, Where It Regulates MCAK. Current Biology, 16(17), pp.1705-1710.

Kohn, K.W. et al., 2012. Gene expression profiles of the NCl-60 human tumor cell lines define molecular interaction networks governing cell migration processes. PLOS ONE, 7(5).

Komarova, Y. et al., 2009. Mammalian end binding proteins control persistent microtubule growth. Journal of Cell Biology, 184(5), pp.691-706.

Kops, G.J.P.L., Foltz, D.R. \& Cleveland, D.W., 2004. Lethality to human cancer cells through massive chromosome loss by inhibition of the mitotic checkpoint. Proceedings of the National Academy of Sciences of the United States of America, 101(23), pp.8699-704.

Krendel, M., Zenke, F.T. \& Bokoch, G.M., 2002. Nucleotide exchange factor GEF-H1 mediates cross-talk between microtubules and the actin cytoskeleton. Nature cell biology, 4(4), pp.294-301.

Krylyshkina, O. et al., 2002. Modulation of substrate adhesion dynamics via microtubule targeting requires kinesin-1. Journal of Cell Biology, 156(2), pp.349-359.

Krylyshkina, O. et al., 2003. Nanometer targeting of microtubules to focal adhesions. Journal of Cell Biology, 161(5), pp.853-859.

Kuroda, S. et al., 1999. Cdc42, Rac1, and their effector IQGAP1 as molecular switches for cadherin-mediated cell-cell adhesion. Biochemical and biophysical research communications, 262(1), pp.1-6. 
Kurokawa, K. \& Matsuda, M., 2005. Localized RhoA Activation as a Requirement for the Induction of Membrane Ruffling. Molecular biology of the cell, 16(8), pp.4294-4303.

Kwon, M. et al., 2015. Direct Microtubule-Binding by Myosin-10 Orients Centrosomes toward Retraction Fibers and Subcortical Actin Clouds. Developmental Cell, 34, pp.1-15.

Labrecque, M.P. et al., 2016. The retinoblastoma protein regulates hypoxia-inducible genetic programs, tumor cell invasiveness and neuroendocrine differentiation in prostate cancer cells. Oncotarget, 5(17).

Lang, G.A. et al., 2004. Gain of function of a p53 hot spot mutation in a mouse model of LiFraumeni syndrome. Cell, 119(6), pp.861-872.

Langermann, S., 1998. Requirement for p53 and p21 to Sustain G 2 Arrest After DNA Damage. Science, 282(November), pp.1497-1502.

Lansbergen, G. et al., 2004. Conformational changes in CLIP-170 regulate its binding to microtubules and dynactin localization. Journal of Cell Biology, 166(7), pp.1003-1014.

Lantz, V.A. \& Miller, K.G., 1998. A class VI unconventional myosin is associated with a homologue of a microtubule-binding protein, cytoplasmic linker protein-170, in neurons and at the posterior pole of Drosophila embryos. Journal of Cell Biology, 140(4), pp.897-910.

Lara-Gonzalez, P., Westhorpe, F.G. \& Taylor, S.S., 2012. The spindle assembly checkpoint. Current Biology, 22(22), pp.R966-R980.

Larsson, N. et al., 1997. Control of microtubule dynamics by oncoprotein 18: dissection of the regulatory role of multisite phosphorylation during mitosis. Molecular and cellular biology, 17(9), pp.5530-5539.

Lauffenburger, D.A. \& Horwitz, A.F., 1996. Cell migration: A physically integrated molecular process. Cell, 84(3), pp.359-369.

Lawson, C.D. \& Burridge, K., 2014. The on-off relationship of Rho and Rac during integrinmediated adhesion and cell migration. Small GTPases, 5(1), pp.37-41.

Lee, S.H. \& Dominguez, R., 2010. Regulation of actin cytoskeleton dynamics in cells. Molecules and cells, 29(4), pp.311-325.

Leibovitz, A. et al., 1976. Classification of human colorectal adenocarcinoma cell lines. Cancer Research, 36(12), pp.4562-4569. 
Lengauer, C., Kinzler, K.W. \& Vogelstein, B., 1997. Genetic instability in colorectal cancers. Nature, 386, pp.623-627.

Leung, T. et al., 1995. A novel serine/threonine kinase binding the ras-related RhoA GTPase which translocates the kinase to peripheral membranes. Journal of Biological Chemistry, 270(49), pp.29051-29054.

Leve, F. \& Morgado-Díaz, J.A., 2012. Rho GTPase signaling in the development of colorectal cancer. Journal of Cellular Biochemistry, 113(8), pp.2549-2559.

Levrero, M. et al., 2000. The p53/p63/p73 family of transcription factors: overlapping and distinct functions. Journal of cell science, 113, pp.1661-1670.

Liao, G., Nagasaki, T. \& Gundersen, G.G., 1995. Low concentrations of nocodazole interfere with fibroblast locomotion without significantly affecting microtubule level: implications for the role of dynamic microtubules in cell locomotion. Journal of cell science, 108, pp.3473-3483.

Ligon, L.A. et al., 2003. The Microtubule Plus-End Proteins EB1 and Dynactin Have Differential Effects on Microtubule Polymerization. Molecular biology of the cell, 14(May), pp.1405-1471.

Liu, Q. et al., 2000. Chk1 is an essential kinase that is regulated by Atr and required for the G2/M DNA damage checkpoint. Genes and Development, 14(12), pp.1448-1459.

Lochter, A. et al., 1997. Matrix metalloproteinase stromelysin-1 triggers a cascade of molecular alterations that leads to stable epithelial-to-mesenchymal conversion and a premalignant phenotype in mammary epithelial cells. Journal of Cell Biology, 139(7), pp.1861-1872.

Löffler, H. et al., 2011. Cep63 recruits Cdk1 to the centrosome: Implications for regulation of mitotic entry, centrosome amplification, and genome maintenance. Cancer Research, 71(6), pp.2129-2139.

Lolli, G. \& Johnson, L.N., 2005. CAK-Cyclin-Dependent Activating Kinase: A key kinase in cell cycle control and a target for Drugs? Cell Cycle, 4(4), pp.572-577.

Losada, A., Hirano, M. \& Hirano, T., 1998. Identification of Xenopus SMC protein complexes required for sister chromatid cohesion. Genes and Development, 12, pp.1966-1997.

De Luca, M. et al., 2008. Aurora-A and ch-TOG act in a common pathway in control of spindle pole integrity. Oncogene, 27(51), pp.6539-6549. 
Machacek, M. et al., 2009. Coordination of Rho GTPase activities during cell protrusion. Nature, 461(7260), pp.99-103.

Mack, N.A. et al., 2011. The diverse roles of Rac signaling in tumorigenesis. Cell Cycle, 10(10), pp.1571-1581.

Malumbres, M. \& Barbacid, M., 2009. Cell cycle, CDKs and cancer: a changing paradigm. Nature reviews. Cancer, 9(3), pp.153-166.

Malumbres, M. \& Barbacid, M., 2005. Mammalian cyclin-dependent kinases. Trends in Biochemical Sciences, 30(11), pp.630-641.

Maney, T. et al., 1998. Mitotic Centromere-associated Kinesin Is Important for Anaphase Chromosome Segregation. J Cell Biol, 142(3), pp.787-801.

Marei, H. \& Malliri, A., 2016. GEFs: Dual regulation of Rac1 signaling. Small GTPases, 0(0), pp.1-10.

Margolis, R.L. \& Wilson, L., 1998. Microtubule treadmilling: What goes around comes around. BioEssays, 20(10), pp.830-836.

Matsuoka, S., Huang, M. \& Elledge, S.J., 1998. Linkage of ATM to cell cycle regulation by the Chk2 protein kinase. Science, 282(5395), pp.1893-1897.

Maxwell, C.A., McCarthy, J. \& Turley, E., 2008. Cell-surface and mitotic-spindle RHAMM: moonlighting or dual oncogenic functions? Journal of cell science, 121, pp.925-932.

Meulmeester, E. \& Ten Dijke, P., 2010. The dynamic roles of TGF-beta in cancer. The Journal of pathology, (October 2010), pp.205-218.

Michaelis, C., Ciosk, R. \& Nasmyth, K., 1997. Cohesins: Chromosomal proteins that prevent premature separation of sister chromatids. Cell, 91(1), pp.35-45.

Mimori-Kiyosue, Y. et al., 2005. CLASP1 and CLASP2 bind to EB1 and regulate microtubule plus-end dynamics at the cell cortex. Journal of Cell Biology, 168(1), pp.141-153.

Mimori-Kiyosue, Y., Shiina, N. \& Tsukita, S., 2000. The dynamic behavior of the APC-binding protein EB1 on the distal ends of microtubules. Current Biology, 10(14), pp.865-868.

Mitsushima, M. et al., 2010. Revolving movement of a dynamic cluster of actin filaments during mitosis. Journal of Cell Biology, 191(3), pp.453-462.

Montenegro-Venegas, C. et al., 2010. MAP1B Regulates Axonal Development by Modulating Rho-GTPase Rac1 Activity. Molecular biology of the cell, 21(22), pp.4042-4056. 
Morin, X. \& Bellaïche, Y., 2011. Mitotic Spindle Orientation in Asymmetric and Symmetric Cell Divisions during Animal Development. Developmental Cell, 21(1), pp.102-119.

Morrison, E.E. et al., 1998. EB1, a protein which interacts with the APC tumour suppressor, is associated with the microtubule cytoskeleton throughout the cell cycle. Oncogene, 17(26), pp.3471-3477.

Muller, P.A.J. \& Vousden, K.H., 2013. P53 Mutations in Cancer. Nature cell biology, 15(1), pp.2-8.

Mullins, R.D. \& Hansen, S.D., 2013. In vitro studies of actin filament and network dynamics. Current Opinion in Cell Biology, 25(1), pp.1-8.

Murphy, K.L., Dennis, A.P. \& Rosen, J.M., 2000. A gain of function p53 mutant promotes both genomic instability and cell survival in a novel p53-null mammary epithelial cell model. Faseb J, 14(14), pp.2291-2302.

Musacchio, A. \& Salmon, E.D., 2007. The spindle-assembly checkpoint in space and time. Nature reviews. Molecular cell biology, 8(5), pp.379-93.

Nabi, I.R., 1999. The polarization of the motile cell. Journal of cell science, 112, pp.18031811.

Nakajima, M. et al., 2007. The complete removal of cohesin from chromosome arms depends on separase. Journal of cell science, 120, pp.4188-4196.

Nalbant, P. et al., 2010. Guanine Nucleotide Exchange Factor-H1 Regulates Cell Migration via Localized Activation of RhoA at the Leading Edge. Molecular biology of the cell, 21(22), pp.4042-4056.

Narumiya, S., Tanji, M. \& Ishizaki, T., 2009. Rho signaling, ROCK and mDia1, in transformation, metastasis and invasion. Cancer and Metastasis Reviews, 28(1-2), pp.6576.

Nezi, L. \& Musacchio, A., 2009. Sister chromatid tension and the spindle assembly checkpoint. Current Opinion in Cell Biology, 21(6), pp.785-795.

Nigg, E.A., 2001. Mitotic kinases as regulators of cell division and its checkpoints. Nature reviews. Molecular cell biology, 2(1), pp.21-32.

Nigg, E.A. \& Stearns, T., 2011. The centrosome cycle: Centriole biogenesis, duplication and inherent asymmetries. Nature Cell Biology, 13(10), pp.1154-1160. 
Nobes, C.D. \& Hall, A., 1995. Rho, Rac, and Cdc42 GTPases regulate the assembly of multimolecular focal complexes associated with actin stress fibers, lamellipodia, and filopodia. Cell, 81(1), pp.53-62.

Noë, V. et al., 2001. Release of an invasion promoter E-cadherin fragment by matrilysin and stromelysin-1. Journal of cell science, 114(114), pp.111-118.

O'Connor, K. \& Chen, M., 2013. Dynamic functions of RhoA in tumor cell migration and invasion. Small GTPases, 4(3), pp.141-147.

O'Connor, K.L., Chen, M. \& Towers, L.N., 2012. Integrin $\alpha 6 \beta 4$ cooperates with LPA signaling to stimulate Rac through AKAP-Lbc-mediated RhoA activation. American journal of physiology. Cell physiology, 302(3), pp.C605-14.

O'Connor, K.L., Nguyen, B.K. \& Mercurio, A.M., 2000. RhoA function in lamellae formation and migration is regulated by the a6ß4 integrin and cAMP metabolism. Journal of Cell Biology, 148(2), pp.253-258.

Ogino, S. et al., 2010. Down-regulation of p21 (CDKN1A/CIP1) is inversely associated with microsatellite instability and $\mathrm{CpG}$ island methylator phenotype (CIMP) in colorectal cancer. The Journal of pathology, 220(September), pp.114-125.

Ohuchi, E. et al., 1997. Membrane-Type metalloproteinase digests extracellular matrix macromolecules including interstitial collagens. Matrix Biology, 16(2), pp.76-77.

Oren, M. \& Rotter, V., 2010. Mutant p53 gain-of-function in cancer. Cold Spring Harbor perspectives in biology, 2(2), p.a001107.

Orr, B. \& Compton, D.A., 2013. A double-edged sword: how oncogenes and tumor suppressor genes can contribute to chromosomal instability. Front Oncol, 3(June), p.164.

Osmani, N. et al., 2010. Cdc42 localization and cell polarity depend on membrane traffic. Journal of Cell Biology, 191(7), pp.1261-1269.

Palamidessi, A. et al., 2008. Endocytic Trafficking of Rac Is Required for the Spatial Restriction of Signaling in Cell Migration. Cell, 134(1), pp.135-147.

Parri, M. \& Chiarugi, P., 2010. Rac and Rho GTPases in cancer cell motility control. Cell communication and signaling : CCS, 8, p.23.

Pellinen, T. \& Ivaska, J., 2006. Integrin traffic. Journal of cell science, 119(Pt 18), pp.37233731 . 
Peters, J.-M., 2006. The anaphase promoting complex/cyclosome: a machine designed to destroy. Nature reviews. Molecular cell biology, 7(9), pp.644-56.

Petitjean, A. et al., 2006. TP53 Impact of Mutant p53 Functional Properties on Mutation Patterns and Tumor Phenotype: Lessons from Recent Developments in the IARC TP53 Database. Human Mutation, 27(July), pp.796-802.

Pinsky, B.A. \& Biggins, S., 2005. The spindle checkpoint: Tension versus attachment. Trends in Cell Biology, 15(9), pp.486-493.

Pollard, T.D. \& Earnshaw, W.C., 2007. Cell Biology. Springer Verlag Berlin Heidelberg

Potter, D.A. et al., 1997. Calpain Regulates Actin Remodeling during Cell Spreading. The Journal of Cel Biology. 139(4), pp.895-905.

Pützer, B.M., 2013. DNp73 : oncotarget in invasion and metastasis. Oncotarget, 5(1), pp.1-2.

Radisky, D.C. et al., 2005. Rac1b and reactive oxygen species mediate MMP-3-induced EMT and genomic instability. Nature, 436(7047), pp.123-7.

Radisky, E.S. \& Radisky, D.C., 2010. Matrix metalloproteinase-induced epithelialmesenchymal transition in breast cancer. Journal of Mammary Gland Biology and Neoplasia, 15(2), pp.201-212.

Rauhala, H.E. et al., 2013. Silencing of the Arp2/3 complex disturbs pancreatic cancer cell migration. Anticancer Research, 33(1), pp.45-52.

Ren, X.D., Kiosses, W.B. \& Schwartz, M.A., 1999. Regulation of the small GTP-binding protein Rho by cell adhesion and the cytoskeleton. EMBO Journal, 18(3), pp.578-585.

Ricke, R.M., van Ree, J.H. \& van Deursen, J.M., 2008. Whole chromosome instability and cancer: a complex relationship. Trends in Genetics, 24(9), pp.457-466.

Ridley, A.J. et al., 2003. Cell migration: integrating signals from front to back. Science, 302(5651), pp.1704-1709.

Ridley, A.J., 2006. Rho GTPases and actin dynamics in membrane protrusions and vesicle trafficking. Trends in Cell Biology, 16(10), pp.522-529.

Ridley, A.J. et al., 1992. The small GTP-binding protein rac regulates growth factor-induced membrane ruffling. Cell, 70(3), pp.401-410. 
Rieder, C.L. et al., 1995. The checkpoint delaying anaphase in response to chromosome monoorientation is mediated by an inhibitory signal produced by unattached kinetochores. Journal of Cell Biology, 130(4), pp.941-948.

Rieder, C.L. \& Salmon, E.D., 1994. Motile kinetochores and polar ejection forces dictate chromosome position on the vertebrate mitotic spindle. Journal of Cell Biology, 124(3), pp.223-233.

Rieder, C.L. \& Salmon, E.D., 1998. The vertebrate cell kinetochore and its roles during mitosis. Trends in Cell Biology, 8(8), pp.310-318.

Rohatgi, R. et al., 1999. The interaction between N-WASP and the Arp2/3 complex links Cdc42-dependent signals to actin assembly. Cell, 97(2), pp.221-231.

Rooney, C. et al., 2010. The Rac activator STEF (Tiam2) regulates cell migration by microtubule-mediated focal adhesion disassembly. EMBO reports, 11(4), pp.292-298.

Rossman, K.L., Der, C.J. \& Sondek, J., 2005. GEF means Go: turning on Rho GTPases with guanine nucleotide-exchange factors. Nature Reviews Molecular Cell Biology, 6(2), pp.167180.

Rudolph-Owen, L.A. et al., 1998. The Matrix Metalloproteinase Matrilysin Influences EarlyStage Mammary Tumorigenesis. Cancer Research, 55(19), pp.5500-5506.

Rufini, A. et al., 2011. p73 in Cancer. Genes and Cancer, 2(4), pp.491-502. A

Sablina, A.A., Chumakov, P.M. \& Kopnin, B.P., 2003. Tumor suppressor p53 and its homologue p73alpha affect cell migration. Journal of Biological Chemistry, 278(30), pp.27362-27371.

Sadok, A. \& Marshall, C.J., 2014. Rho GTPases: Masters of Cell Migration. Small GTPases, 5(4).

Sahai, E., 2005. Mechanisms of cancer cell invasion. Current opinion in genetics \& development, 15(1), pp.87-96.

Salaycik, K.J. et al., 2005. Quantification of microtubule nucleation, growth and dynamics in wound-edge cells. Journal of cell science, 118(Pt 18), pp.4113-4122.

Sanchez, Y., 1997. Conservation of the Chk1 Checkpoint Pathway in Mammals: Linkage of DNA Damage to Cdk Regulation Through Cdc25. Science, 277(5331), pp.1497-1501. 
Sanhaji, M. et al., 2011. Mitotic centromere-associated kinesin (MCAK): a potential cancer drug target. Oncotarget, 2(12), pp.935-47.

Schmidt, J.C. et al., 2012. The Kinetochore-Bound Ska1 Complex Tracks Depolymerizing Microtubules and Binds to Curved Protofilaments. Developmental Cell, 23(5), pp.968-980.

Schmidt, M. et al., 2000. Differential modulation of paclitaxel-mediated apoptosis by p21 Waf1 and p27 Kip1. Oncogene, 19, pp.2423-2429.

Schmidt, S. \& Debant, A., 2014. Function and regulation of the Rho guanine nucleotide exchange factor Trio. Small GTPases, 5(June), p.e29769.

Schober, J.M. et al., 2009. Migration and actin protrusion in melanoma cells are regulated by EB1 protein. Cancer Letters, 284(1), pp.30-36.

Schuyler, S.C. \& Pellman, D., 2001. Microtubule "plus-end-tracking proteins": The end is just the beginning. Cell, 105(4), pp.421-424.

Semple, T.U. et al., 1978. Tumor and Lymphoid Cell Lines from a Patient with Carcinoma of the Colon for a Cytotoxicity Model. Cancer Research, 38(MAY), pp.1345-1355.

Sheltzer, J.M., 2013. A transcriptional and metabolic signature of primary aneuploidy is present in chromosomally-unstable cancer cell and informs clinical prognosis. Cancer research, 73(21), pp.6401-6412.

Sheltzer, J.M. \& Amon, A., 2011. The aneuploidy paradox: Costs and benefits of an incorrect karyotype. Trends in Genetics, 27(11), pp.446-453.

Shiota, M. et al., 2008. Twist and p53 reciprocally regulate target genes via direct interaction. Oncogene, 27(42), pp.5543-5553.

Siegrist, S.E. \& Doe, C.Q., 2007. Microtubule-induced cortical cell polarity. Genes \& Development, 21(5), pp.483-496.

Smit, M.A. \& Peeper, D.S., 2008. Deregulating EMT and Senescence: Double Impact by a Single Twist. Cancer Cell, 14(1), pp.5-7.

Spaderna, S. et al., 2006. A Transient, EMT-Linked Loss of Basement Membranes Indicates Metastasis and Poor Survival in Colorectal Cancer. Gastroenterology, 131(3), pp.830-840.

Steder, M. et al., 2013. DNp73 Exerts Function in Metastasis Initiation by Disconnecting the Inhibitory Role of EPLIN on IGF1R-AKT/STAT3 Signaling. Cancer Cell, 24(4), pp.512-527. 
Stepanova, T. et al., 2003. Visualization of microtubule growth in cultured neurons via the use of EB3-GFP (end-binding protein 3-green fluorescent protein). The Journal of neuroscience : the official journal of the Society for Neuroscience, 23(7), pp.2655-2664.

Straube, A. \& Merdes, A., 2007. EB3 Regulates Microtubule Dynamics at the Cell Cortex and Is Required for Myoblast Elongation and Fusion. Current Biology, 17(15), pp.1318-1325.

Suardet, L. et al., 1992. Responsiveness of three newly established human colorectal cancer cell lines to transforming growth factors beta 1 and beta 2. Cancer research, 52(13), pp.3705-3712.

Sumara, I. et al., 2000. Characterization of vertebrate cohesin complexes and their regulation in prophase. Journal of Cell Biology, 151(4), pp.749-761.

Talos, F. et al., 2007. p73 Suppresses Polyploidy and Aneuploidy in the Absence of Functional p53. Molecular Cell, 27(4), pp.647-659.

Tamura, N. \& Draviam, V.M., 2012. Microtubule plus-ends within a mitotic cell are "moving platforms" with anchoring, signalling and force-coupling roles. Open biology, 2(11), p.120132.

Tanenbaum, M.E. et al., 2008. Dynein, Lis1 and CLIP-170 counteract Eg5-dependent centrosome separation during bipolar spindle assembly. The EMBO journal, 27(24), pp.3235-45.

Tanenbaum, M.E. \& Medema, R.H., 2010. Mechanisms of Centrosome Separation and Bipolar Spindle Assembly. Developmental Cell, 19(6), pp.797-806.

Tarapore, P. \& Fukasawa, K., 2002. Loss of p53 and centrosome hyperamplification. Oncogene, 21(40), pp.6234-40.

Théry, M. et al., 2005. The extracellular matrix guides the orientation of the cell division axis. Nature cell biology, 7(10), pp.947-953.

Thiery, J.P. \& Sleeman, J.P., 2006. Complex networks orchestrate epithelial-mesenchymal transitions. Nature reviews. Molecular cell biology, 7(2), pp.131-42.

Thompson, S.L., Bakhoum, S.F. \& Compton, D.A., 2010. Mechanisms of Chromosomal Instability. Current Biology, 20(6), pp.R285-R295.

Thompson, S.L. \& Compton, D.A., 2008. Examining the link between chromosomal instability and aneuploidy in human cells. Journal of Cell Biology, 180(4), pp.665-672. 
Thukral, S.K. et al., 1995. Discrimination of DNA binding sites by mutant p53 proteins. Molecular and cellular biology, 15(9), pp.5196-202.

Tighe, A. et al., 2001. Aneuploid colon cancer cells have a robust spindle checkpoint. EMBO Reports, 2(7), pp.609-614.

Torres, E.M. et al., 2007. Effects of aneuploidy on cellular physiology and cell division in haploid yeast. Science, 317(5840), pp.916-24.

Toyoshima, F. \& Nishida, E., 2007. Integrin-mediated adhesion orients the spindle parallel to the substratum in an EB1- and myosin X-dependent manner. The EMBO journal, 26(6), pp.1487-1498.

Tran, A.D.A. et al., 2007. HDAC6 deacetylation of tubulin modulates dynamics of cellular adhesions. Journal of cell science, 120, pp.1469-1479.

Tsvetkov, A.S. et al., 2007. Microtubule-binding proteins CLASP1 and CLASP2 interact with actin filaments. Cell Motility and the Cytoskeleton, 64(7), pp.519-530.

Tuve, S. et al., 2004. Alterations of dTA-p73 splice transcripts during melanoma development and progression. International Journal of Cancer, 108(1), pp.162-166.

Uramoto, H. et al., 2004. Expression of $\Delta$ Np73 Predicts Poor Prognosis in Lung Cancer Expression. Clinical Cancer Research, 10, pp.6905-6911.

Uzbekov, R., Kireyev, I. \& Prigent, C., 2002. Centrosome separation: Respective role of microtubules and actin filaments. Biology of the Cell, 94(4-5), pp.275-288.

Vasiliev, J.M. et al., 1970. Effect of colcemid on the locomotory behaviour of fibroblasts. Journal of embryology and experimental morphology, 24(3), pp.625-640.

Vaughan, K.T., 2005. TIP maker and TIP marker; EB1 as a master controller of microtubule plus ends. Journal of Cell Biology, 171(2), pp.197-200.

Vega, F.M. et al., 2011. RhoA and RhoC have distinct roles in migration and invasion by acting through different targets. Journal of Cell Biology, 193(4), pp.655-665.

Wang, S.-P. et al., 2009. p53 controls cancer cell invasion by inducing the MDM2-mediated degradation of Slug. Nature cell biology, 11(6), pp.694-704.

Wang, W. et al., 2008. Centrosome separation driven by actin-microfilaments during mitosis is mediated by centrosome-associated tyrosine-phosphorylated cortactin. Journal of cell science, 121(Pt 8), pp.1334-43. 
Wang, W. et al., 2004. Identification and testing of a gene expression signature of invasive carcinoma cells within primary mammary tumors. Cancer Research, 64(23), pp.8585-8594.

Wang, Y. et al., 2005. Overexpression of EB1 in human esophageal squamous cell carcinoma (ESCC) may promote cellular growth by activating beta-catenin/TCF pathway. Oncogene, 24(44), pp.6637-45.

Watanabe, T. et al., 2004. Interaction with IQGAP1 links APC to Rac1, Cdc42, and actin filaments during cell polarization and migration. Developmental Cell, 7(6), pp.871-883.

Waterman-Storer, C.M. et al., 1999. Microtubule growth activates Rac1 to promote lamellipodial protrusion in fibroblasts. Nature cell biology, 1(1), pp.45-50.

Waterman-Storer, C.M. \& Salmon, E., 1999. Positive feedback interactions between microtubule and actin dynamics during cell motility. Current opinion in cell biology, 11(1), pp.61-67.

Waterman-Storer, C.M. \& Salmon, E.D., 1997. Actomyosin-based retrograde flow of microtubules in the lamella of migrating epithelial cells influences microtubule dynamic instability and turnover and is associated with microtubule breakage and treadmilling. Journal of Cell Biology, 139(2), pp.417-434.

Watson, P. \& Stephens, D.J., 2006. Microtubule plus-end loading of p150(Glued) is mediated by EB1 and CLIP-170 but is not required for intracellular membrane traffic in mammalian cells. Journal of cell science, 119, pp.2758-2767.

Weber, K.L. et al., 2004. A microtubule-binding myosin required for nuclear anchoring and spindle assembly. Nature, 431(7006), pp.325-329.

Wen, Y. et al., 2004. EB1 and APC bind to mDia to stabilize microtubules downstream of Rho and promote cell migration. Nature cell biology, 6(9), pp.820-830.

Whalley, H.J. et al., 2015. Cdk1 phosphorylates the Rac activator Tiam1 to activate centrosomal Pak and promote mitotic spindle formation. Nature Communications, 6(May), p.7437.

Whitehead, C.M. \& Rattner, J.B., 1998. Expanding the role of HsEg5 within the mitotic and post-mitotic phases of the cell cycle. Journal of cell science, 111 ( Pt 1, pp.2551-2561.

Wilhelm, T. et al., 2013. Spontaneous slow replication fork progression elicits mitosis alterations in homologous recombination-deficient mammalian cells. Proceedings of the National Academy of Sciences of the United States of America, 111(2), p.201311520. 
Wittmann, T., Bokoch, G.M. \& Waterman-Storer, C.M., 2003. Regulation of leading edge microtubule and actin dynamics downstream of Rac1. Journal of Cell Biology, 161(5), pp.845-851.

Wittmann, T., Hyman, A. \& Desai, A., 2001. The spindle: a dynamic assembly of microtubules and motors. Nature cell biology, 3(1), pp.E28-E34.

Wittmann, T. \& Waterman-Storer, C.M., 2001. Cell motility: can Rho GTPases and microtubules point the way? Journal of cell science, 114, pp.3795-3803.

Woodcock, S.A. et al., 2010. Tiam1-Rac Signaling Counteracts Eg5 during Bipolar Spindle Assembly to Facilitate Chromosome Congression. Current Biology, 20(7), pp.669-675.

Woolner, S. et al., 2008. Myosin-10 and actin filaments are essential for mitotic spindle function. Journal of Cell Biology, 182(1), pp.77-88.

Yamada, K.M. \& Araki, M., 2001. Tumor suppressor PTEN: modulator of cell signaling, growth, migration and apoptosis. Journal of cell science, 114(Pt 13), pp.2375-2382.

Yamaguchi, H., Lorenz, M., et al., 2005. Molecular mechanisms of invadopodium formation: The role of the N-WASP-Arp2/3 complex pathway and cofilin. Journal of Cell Biology, 168(3), pp.441-452.

Yamaguchi, H. \& Condeelis, J., 2007. Regulation of the actin cytoskeleton in cancer cell migration and invasion. Biochimica et Biophysica Acta - Molecular Cell Research, 1773(5), pp.642-652.

Yamaguchi, H., Wyckoff, J. \& Condeelis, J., 2005. Cell migration in tumors. Current Opinion in Cell Biology, 17, pp.559-564.

Yamakita, Y. et al., 2003. Caldesmon inhibits Arp2/3-mediated actin nucleation. Journal of Biological Chemistry, 278(20), pp.17937-17944.

Yang, A. et al., 1998. P63, a P53 Homolog At 3Q27-29, Encodes Multiple Products With Transactivating, Death-Inducing, and Dominant-Negative Activities. Molecular Cell, 2(3), pp.305-16.

Yang, A. \& McKeon, F., 2000. P63 and P73: P53 mimics, menaces and more. Nature reviews. Molecular cell biology, 1(3), pp.199-207.

Yeh, S. et al., 1994. Frequent Genetic Alterations at the Distal Region of Chromosome ip in Human Hepatocellular Carcinomas. Cancer Research, 54, pp.4188-4192. 
Yeo, C.Q.X. et al., 2016. P53 Maintains Genomic Stability by Preventing Interference between Transcription and Replication. Cell Reports, 15(1), pp.132-146.

Yoshida, T. et al., 2010. Blockade of Rac1 activity induces G1 cell cycle arrest or apoptosis in breast cancer cells through downregulation of cyclin D1, survivin, and X-linked inhibitor of apoptosis protein. Molecular cancer therapeutics, 9(6), pp.1657-1668.

Yoshio, T. et al., 2007. Caldesmon suppresses cancer cell invasion by regulating podosome/invadopodium formation. FEBS Letters, 581(20), pp.3777-3782.

Zaika, A.I. et al., 2002. DeltaNp73, a dominant-negative inhibitor of wild-type p53 and TAp73, is up-regulated in human tumors. The Journal of experimental medicine, 196(6), pp.765-780.

Zhang, J. et al., 2005. Silencing p21(Waf1/Cip1/Sdi1) expression increases gene transduction efficiency in primitive human hematopoietic cells. Gene therapy, 12(19), pp.1444-1452.

Zhang, Y. et al., 2012. Mammary epithelial cell polarity is regulated differentially by p73 isoforms via epithelial-to-mesenchymal transition. Journal of Biological Chemistry, 287(21), pp.17746-17753.

Zheng, H.C. et al., 2008. Arp2/3 overexpression contributed to pathogenesis, growth and invasion of gastric carcinoma. Anticancer Research, 28(4 B), pp.2225-2232.

Zheng, Y., 2001. Dbl family guanine nucleotide exchange factors. Trends in Biochemical Sciences, 26(12), pp.724-732.

Van Zijl, F., Krupitza, G. \& Mikulits, W., 2011. Initial steps of metastasis: Cell invasion and endothelial transmigration. Mutation Research - Reviews in Mutation Research, 728(1-2), pp.23-34. 


\section{Acknowledgement}

At this point, I would like to thank everyone who contributed to this thesis and who supported me during the last four years.

First of all, I want to thank my doctorate supervisor Prof. Dr. Holger Bastians for giving me the opportunity to work on this exciting topic in his lab. Thanks for the constant guidance as well as valuable ideas and discussions on the project.

Besides, I would like to thank my thesis committee members Prof. Dr. Michael Thumm and Prof. Dr. Dieter Kube for their comments and suggestions throughout my project.

Furthermore, I thank Prof. Dr. Heike Krebber for providing working materials. I also want to thank Prof. Dr. Jörg Wilting and his lab members, especially Berti Manshausen and Sonja Schwoch, for introducing and supervising the CAM assays, providing the main working materials and supporting the processing and analyses of the samples. Thanks to Silvia von der Heyde for bioinformatical analyses of the RNA sequencing results and to Prof. Dr. Maik Kschischo for discussion on statistical tests.

I am extremely grateful to all current and former members of the Bastians lab for the fantastic working atmosphere, constant support, motivation and all the fun we had during and after working in the lab. I want to thank Dr. Norman Ertych and Dr. Ailine Stolz for sharing their knowledge and teaching me the essential techniques. Thanks to Nadine Schermuly, Elina Glaubke and Magdalena Kistner for vivid discussions on failed and successive experiments and also all the non-scientific related topics as well as constructive coffee breaks and cocktail hours. Further, I would particularly like to thank Dr. Sina Lüddecke for reading and commenting on the manuscript, cheery words whenever necessary and being a friend also outside the lab.

Last but not least, I want to express my special and greatest appreciation to my family for practically and morally supporting me throughout my thesis and my life in general. I want to thank them for their continuous love and motivation. I could not have done it without them. 


\section{Curriculum Vitae}

\section{Personal Data}

\section{Katharina Berger}

Born on $11^{\text {th }}$ of January 1988

in Brandenburg an der Havel

\section{School Education}

08/94-06/00

Konrad-Sprengel-Grundschule in Brandenburg an der Havel

08/00-06/07

Bertolt-Brecht-Gymnasium in Brandenburg an der Havel

\section{Scientific Education}

$10 / 07-09 / 10$

Bachelor of Science in Life Sciences

University of Potsdam

Bachelor Thesis

Institute for Molecular Biology

"Analysis of $\mathrm{H}_{2} \mathrm{O}_{2}$ responsive gene expression in Arabidopsis thaliana"

$10 / 10-08 / 12$

Master of Science in Biochemistry and Molecular Biology

University of Potsdam

Master Thesis

Institute for Cell Biology

"Functional Characterization of CP55 in Dictyostelium"

since $10 / 12$

PhD student in the group of Prof. Dr. Holger Bastians, Institute for Molecular Oncology

Section of Cellular Oncology

University Medical School

Member of the GGNB program "Molecular Biology of Cells" 